\title{
Status of muon collider research and development and future plans
}

Charles M. Ankenbrandt, ${ }^{1}$ Muzaffer Atac, ${ }^{1}$ Bruno Autin, ${ }^{2}$ Valeri I. Balbekov, ${ }^{1}$ Vernon D. Barger, ${ }^{3}$ Odette Benary, ${ }^{4}$ J. Scott Berg, ${ }^{5}$ Michael S. Berger, ${ }^{5}$ Edgar L. Black,${ }^{6}$ Alain Blondel, ${ }^{7}$ S. Alex Bogacz, ${ }^{8}$ T. Bolton, ${ }^{9}$ Shlomo Caspi, ${ }^{10}$

Christine Celata, ${ }^{10}$ Weiren Chou, ${ }^{1}$ David B. Cline, ${ }^{11}$ John Corlett, ${ }^{10}$ Lucien Cremaldi, ${ }^{12}$ H. Thomas Diehl, ${ }^{1}$ Alexandr Drozhdin, ${ }^{1}$ Richard C. Fernow, ${ }^{13}$ David A. Finley, ${ }^{1}$ Yasuo Fukui, ${ }^{11}$ Miguel A. Furman, ${ }^{10}$ Tony Gabriel,,${ }^{15}$ Juan C. Gallardo, ${ }^{13}$, Alper A. Garren, ${ }^{11}$ Stephen H. Geer, ${ }^{1}$ Ilya F. Ginzburg, ${ }^{16}$ Michael A. Green, ${ }^{10}$ Hulya Guler, ${ }^{17}$ John F. Gunion, ${ }^{18}$ Ramesh Gupta, ${ }^{10}$ Tao Han, ${ }^{3}$ Gail G. Hanson, ${ }^{5}$ Ahmed Hassanein, ${ }^{19}$ Norbert Holtkamp, ${ }^{1}$ Colin Johnson, ${ }^{2}$ Carol Johnstone, ${ }^{1}$ Stephen A. Kahn, ${ }^{13}$ Daniel M. Kaplan, ${ }^{6}$ Eun San Kim, ${ }^{10}$ Bruce J. King, ${ }^{13}$ Harold G. Kirk, ${ }^{13}$ Yoshitaka Kuno, ${ }^{14}$ Paul Lebrun, ${ }^{1}$ Kevin Lee, ${ }^{11}$ Peter Lee, ${ }^{10}$ Derun Li, ${ }^{10}$ David Lissauer, ${ }^{13}$ Laurence S. Littenberg, ${ }^{13}$ Changguo Lu, ${ }^{17}$ Alfredo Luccio, ${ }^{13}$ Joseph D. Lykken, ${ }^{1}$ Kirk T. McDonald,${ }^{17}$ Alfred D. McInturff, ${ }^{10}$ John R. Miller, ${ }^{20}$ Frederick E. Mills, ${ }^{1}$ Nikolai V. Mokhov, ${ }^{1}$ Alfred Moretti, ${ }^{1}$ Yoshiharu Mori, ${ }^{14}$

David V. Neuffer, ${ }^{1}$ King-Yuen Ng, ${ }^{1}$ Robert J. Noble, ${ }^{1}$ James H. Norem, ${ }^{19,1}$ Yasar Onel, ${ }^{21}$ Robert B. Palmer, ${ }^{13}$ Zohreh Parsa, ${ }^{13}$ Yuriy Pischalnikov, ${ }^{11}$ Milorad Popovic, ${ }^{1}$ Eric J. Prebys,${ }^{17}$ Zubao Qian, ${ }^{1}$ Rajendran Raja, ${ }^{1}$ Claude B. Reed, ${ }^{19}$ Pavel Rehak, ${ }^{13}$ Thomas Roser, ${ }^{13}$ Robert Rossmanith, ${ }^{22}$ Ronald M. Scanlan, ${ }^{10}$ Andrew M. Sessler, ${ }^{10}$ Brad Shadwick, ${ }^{23}$ Quan-Sheng Shu, ${ }^{8}$ Gregory I. Silvestrov, ${ }^{24}$ Alexandr N. Skrinsky, ${ }^{24}$ Dale Smith, ${ }^{19}$

Panagiotis Spentzouris, ${ }^{1}$ Ray Stefanski, ${ }^{1}$ Sergei Striganov, ${ }^{1}$ Iuliu Stumer,${ }^{13}$ Don Summers, ${ }^{12}$ Valeri Tcherniatine, ${ }^{13}$ Lee C. Teng, ${ }^{19}$ Alvin V. Tollestrup, ${ }^{1}$ Yağmur Torun, ${ }^{13,25}$ Dejan Trbojevic, ${ }^{13}$ William C. Turner,${ }^{10}$ Sven E. Vahsen, ${ }^{17}$ Andreas Van Ginneken, ${ }^{1}$ Tatiana A. Vsevolozhskaya, ${ }^{24}$ Weishi Wan, ${ }^{1}$ Haipeng Wang, ${ }^{13}$ Robert Weggel,,${ }^{13}$ Erich H. Willen, ${ }^{13}$ Edmund J. N. Wilson, ${ }^{2}$ David R. Winn, ${ }^{26}$ Jonathan S. Wurtele, ${ }^{23}$ Takeichiro Yokoi, ${ }^{14}$ Yongxiang Zhao, ${ }^{13}$ and Max Zolotorev ${ }^{10}$

(Muon Collider Collaboration)

${ }^{1}$ Fermi National Accelerator Laboratory, P.O. Box 500, Batavia, Illinois 60510

${ }^{2}$ CERN, 1211 Geneva 23, Switzerland

${ }^{3}$ Department of Physics, University of Wisconsin, Madison, Wisconsin 53706

${ }^{4}$ Tel-Aviv University, Ramat-Aviv, Tel-Aviv 69978, Israel

${ }^{5}$ Physics Department, Indiana University, Bloomington, Indiana 47405

${ }^{6}$ Physics Division, Illinois Institute of Technology, Chicago, Illinois 60616

${ }^{7}$ École Polytechnique, Laboratoire de Physique Nucléaire et de Hautes Energies, Palaiseau F-91128, France

${ }^{8}$ Jefferson Laboratory, 12000 Jefferson Avenue, Newport News, Virginia 23606

${ }^{9}$ Kansas State University, Manhattan, Kansas 66502-2601

${ }^{10}$ Lawrence Berkeley National Laboratory, 1 Cyclotron Road, Berkeley, California 94720

${ }^{11}$ University of California Los Angeles, Los Angeles, California 90095

${ }^{12}$ University of Mississippi, Oxford, Mississippi 38677

${ }^{13}$ Brookhaven National Laboratory, Upton, New York 11973

${ }^{14}$ KEK High Energy Accelerator Research Organization, 1-1 Oho, Tsukuba 305, Japan

${ }^{15}$ Oak Ridge National Laboratory, Oak Ridge, Tennessee 37831

${ }^{16}$ Institute of Mathematics, 630090 Novosibirsk, Russia

${ }^{17}$ Joseph Henry Laboratories, Princeton University, Princeton, New Jersey 08544

${ }^{18}$ Physics Department, University of California, Davis, California 95616

${ }^{19}$ Argonne National Laboratory, Argonne, Illinois 60439

${ }^{20}$ Magnet Science \& Technology, National High Magnetic Field Laboratory, Tallahassee, Florida 32310

${ }^{21}$ Physics Department, Van Allen Hall, University of Iowa, Iowa City, Iowa 52242

${ }^{22}$ Research Center Karlsruhe, D-76021 Karlsruhe, Germany

${ }^{23}$ Physics Department, University of California, Berkeley, California 94720

${ }^{24}$ Budker Institute of Nuclear Physics, 630090 Novosibirsk, Russia

${ }^{25}$ Department of Physics and Astronomy, SUNY, Stony Brook, New York 11790

${ }^{26}$ Fairfield University, Fairfield, Connecticut 06430

(Received 21 December 1998; published 3 August 1999)

The status of the research on muon colliders is discussed and plans are outlined for future theoretical and experimental studies. Besides work on the parameters of a 3-4 and $0.5 \mathrm{TeV}$ center-of-mass (COM) energy collider, many studies are now concentrating on a machine near $0.1 \mathrm{TeV}$ (COM) that could be a factory for the $s$-channel production of Higgs particles. We discuss the research on the various components in such muon colliders, starting from the proton accelerator needed to generate pions from 


\begin{abstract}
a heavy- $Z$ target and proceeding through the phase rotation and decay $\left(\pi \rightarrow \mu \nu_{\mu}\right)$ channel, muon cooling, acceleration, storage in a collider ring, and the collider detector. We also present theoretical and experimental R\&D plans for the next several years that should lead to a better understanding of the design and feasibility issues for all of the components. This report is an update of the progress on the research and development since the feasibility study of muon colliders presented at the Snowmass '96 Workshop [R. B. Palmer, A. Sessler, and A. Tollestrup, Proceedings of the 1996 DPF/DPB Summer Study on High-Energy Physics (Stanford Linear Accelerator Center, Menlo Park, CA, 1997)].
\end{abstract}

PACS numbers: 13.10.+q, 14.60.Ef, 29.27.-a, 29.20.Dh

\section{INTRODUCTION}

The standard model of electroweak and strong interactions has passed precision experimental tests at the highest energy scale accessible today. Theoretical arguments indicate that new physics beyond the standard model associated with the electroweak gauge symmetry breaking and fermion mass generation will emerge in parton collisions at or approaching the $\mathrm{TeV}$ energy scale. It is likely that both hadron-hadron and lepton-antilepton colliders will be required to discover and make precision measurements of the new phenomena. The next big step forward in advancing the hadron-hadron collider energy frontier will be provided by the CERN Large Hadron Collider (LHC), a proton-proton collider with a center-of-mass (COM) energy of $14 \mathrm{TeV}$ which is due to come into operation in 2005. Note that in a high energy hadron beam, valence quarks carry momenta which are, approximately, between $\frac{1}{6}$ and $\frac{1}{9}$ of the hadron momentum. The LHC will therefore provide hard parton-parton collisions with typical center-of-mass energies of $2.3-1.5 \mathrm{TeV}$.

The route toward $\mathrm{TeV}$-scale lepton-antilepton colliders is less clear. The lepton-antilepton colliders built so far have been $e^{+} e^{-}$colliders, such as the Large Electron Positron Collider (LEP) at CERN and the Stanford Linear Collider (SLC) at SLAC. In a circular ring such as LEP, the energy lost per revolution in $\mathrm{keV}$ is $88.5 \times E^{4} / \rho$, where the electron energy $E$ is in $\mathrm{GeV}$, and the radius of the orbit $\rho$ is in meters. Hence, the energy loss grows rapidly as $E$ increases. This limits the centerof-mass energy that would be achievable in a LEP-like collider. The problem can be avoided by building a linear machine (the SLC is partially linear), but, with current technologies, such a machine must be very long (30$40 \mathrm{~km}$ ) to attain the $\mathrm{TeV}$ energy scale. Even so, radiation during the beam-beam interaction (beamstrahlung) limits the precision of the COM energy [1].

For a lepton with mass $m$ the radiative energy losses are inversely proportional to $\mathrm{m}^{4}$. Hence, the energyloss problem can be solved by using heavy leptons. In practice this means using muons, which have a mass $\approx 207$ times that of an electron. The resulting reduction in

\footnotetext{
*Corresponding author.

Email address: gallardo@bnl.gov
}

radiative losses enables higher energies to be reached and smaller collider rings to be used [2,3]. Parameters for 10$100 \mathrm{TeV}$ colliders have been discussed [4,5]. Estimated sizes of the accelerator complexes required for $0.1,0.5$, and $4 \mathrm{TeV}$ muon colliders are compared with the sizes of other possible future colliders, and with the FNAL and BNL sites in Fig. 1. Note that muon colliders with COM energies up to $\approx 4 \mathrm{TeV}$ would fit on these existing laboratory sites. The cost of building a muon collider is not yet known. However, since muon colliders are relatively small, they may be significantly less expensive than alternative machines.

Since muons decay quickly, large numbers of them must be produced to operate a muon collider at high luminosity. Collection of muons from the decay of pions produced in proton-nucleus interactions results in a large initial phase volume for the muons, which must be reduced (cooled) by a factor of $10^{6}$ for a practical collider. This may be compared with the antiproton stochastic cooling achieved in the Tevatron. In this case the $6 \mathrm{D}$ phase space is reduced by approximately a factor of $10^{6}$, while with stacking the phase space density [6,7] is increased by a factor of $10^{10}$. The technique of ionization cooling is proposed for the $\mu^{+} \mu^{-}$collider [8-11]. This technique is uniquely applicable to muons because of their minimal interaction with matter.

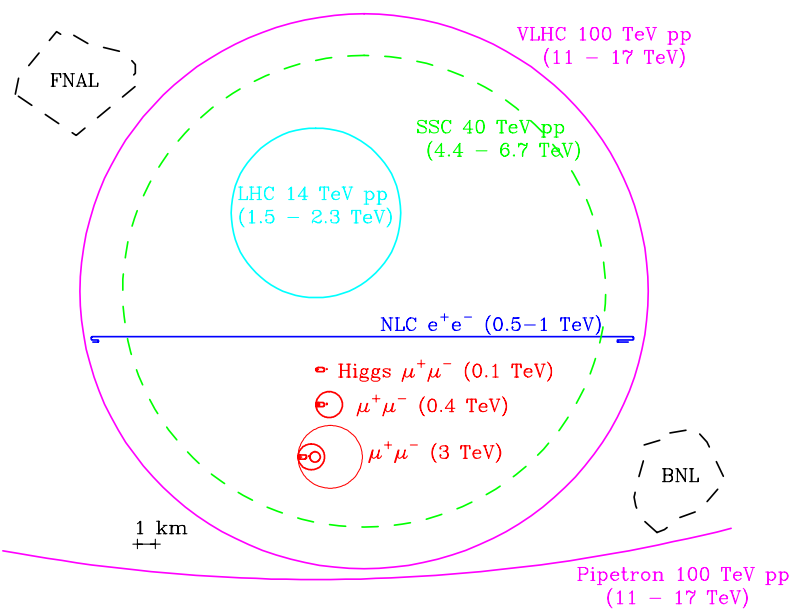

FIG. 1. (Color) Comparative sizes of various proposed high energy colliders compared with the FNAL and BNL sites. The energies in parentheses give for lepton colliders their COM energies and for hadron colliders the approximate range of COM energies attainable for hard parton-parton collisions. 
Muon colliders also offer some significant physics advantages. The small radiative losses permit very small beam-energy spreads to be achieved. For example, momentum spreads as low as $\Delta P / P=0.003 \%$ are believed to be possible for a low energy collider. By measuring the time-dependent decay asymmetry resulting from the naturally polarized muons, it has been shown [12] that the beam energy could be determined with a precision of $\Delta E / E=10^{-6}$. The small beam-energy spread, together with the precise energy determination, would facilitate measurements of the masses and widths of any new resonant states scanned by the collider. In addition, since the cross section for producing a Higgs-like scalar particle in the $s$ channel (direct lepton-antilepton annihilation) is proportional to $\mathrm{m}^{2}$, this extremely important process could be studied only at a muon collider and not at an $e^{+} e^{-}$collider [13]. Finally, the decaying muons will produce copious quantities of neutrinos. Even short straight sections in a muon collider ring will result in neutrino beams several orders of magnitude higher in intensity than presently available, permitting greatly extended studies of neutrino oscillations, nucleon structure functions, the Cabibbo-Kobayashi-Maskawa matrix, and precise indirect measurements of the $W$-boson mass [14] (see Sec. II I).

The concept of muon colliders was introduced by Budker [2,3] and developed further by Skrinsky et al. [15-22] and Neuffer [13,23-25]. They pointed out the significant challenges in designing an accelerator complex that can make, accelerate, and collide $\mu^{+}$and $\mu^{-}$bunches all within the muon lifetime of $2.2 \mu \mathrm{s}(c \tau=659 \mathrm{~m})$. A concerted study of a muon collider design has been underway in the U.S. since 1992 [26-42]. By the Sausalito workshop [30] in 1995, it was realized that with new ideas and modern technology it may be feasible to make muon bunches containing a few times $10^{12}$ muons, compress their phase space, and accelerate them up to the multi-TeV energy scale before more than about $3 / 4$ of them have decayed. With careful design of the collider ring and shielding, it appears possible to reduce to acceptable levels the backgrounds within the detector that arise from the very large flux of electrons produced in muon decays. These realizations led to an intense activity, which resulted in the muon collider feasibility study report $[43,44]$ prepared for the 1996 DPF/DPB Summer Study on High-Energy Physics (the Snowmass '96 Workshop). Since then, the physics prospects at a muon collider have been studied extensively [45-47], and the potential physics program at a muon collider facility has been explored in workshops [39] and conferences [40].

Encouraged by further progress in developing the muon collider concept, together with the growing interest and involvement of the high-energy-physics community, the Muon Collider Collaboration became a formal entity in May of 1997. The Collaboration is led by an executive board with members from Brookhaven National
Laboratory (BNL), Fermi National Accelerator Laboratory (FNAL), Lawrence Berkeley National Laboratory (LBNL), Budker Institute for Nuclear Physics (BINP), University of California at Los Angeles (UCLA), University of Mississippi, and Princeton University. The goal of the Collaboration is to complete within a few years the R\&D needed to determine whether a muon collider is technically feasible, and, if it is, to design the first muon collider (FMC).

Table I gives the parameters of the muon colliders under study [48-55], which have COM energies of 0.1 , 0.4 , and $3 \mathrm{TeV}$, and Figs. 2 and 3 show possible outlines of the 0.1 and $3 \mathrm{TeV}$ machines. In the former case, parameters are given in the table for operation with three different beam-energy spreads: $\Delta p / p=0.12 \%, 0.01 \%$, and $0.003 \%$. In all cases, proton bunches containing $2.5-5 \times 10^{13}$ particles are accelerated to energies of 16 $\mathrm{GeV}$. The protons interact in a target to produce $\mathcal{O}\left(10^{13}\right)$ charged pions of each sign. A large fraction of these pions can be captured in a high-field solenoid. Muons are produced by allowing the pions to decay into a lowerfield solenoidal channel. To collect as many particles as possible within a useful energy interval, rf cavities are used to accelerate the lower energy particles and decelerate the higher energy particles (so-called phase rotation). With two proton bunches every accelerator cycle, the first used to make and collect positive muons and the second to make and collect negative muons, there are about $10^{13}$ muons of each charge available at the end of the decay channel per accelerator cycle. If the proton accelerator is cycling at $15 \mathrm{~Hz}$, then, in an operational year $\left(10^{7} \mathrm{~s}\right)$, about $10^{21}$ positive and negative muons would be produced and collected.

As stated before, the muons exiting the decay channel populate a very diffuse phase space. The next step in the muon collider complex is to cool the muon bunch, i.e., to turn the diffuse muon cloud into a very bright bunch with small longitudinal and transverse dimensions, suitable for accelerating and injecting into a collider. The cooling must be done within a time that is short compared to the muon lifetime. Conventional cooling techniques (stochastic cooling [56] and electron cooling [16]) take too long. The technique proposed for cooling muons is called ionization cooling $[8,10,11]$, and will be discussed in detail in Sec. V. Briefly, the muons traverse some material in which they lose both longitudinal and transverse momentum by ionization losses $(d E / d x)$. The longitudinal momentum is then replaced using an $\mathrm{rf}$ accelerating cavity, and the process is repeated many times until there is a large reduction in the transverse phase space occupied by the muons. The energy spread within the muon beam can also be reduced by using a wedge-shaped absorber in a region of dispersion (where the transverse position is momentum dependent). The wedge is arranged so that the higher energy particles pass through more material than lower energy 
TABLE I. Baseline parameters for high energy and low energy muon colliders. Higgs/yr assumes a cross section $\sigma=$ $5 \times 10^{4} \mathrm{fb}$; a Higgs width $\Gamma=2.7 \mathrm{MeV}, 1 \mathrm{yr}=10^{7} \mathrm{~s}$.

\begin{tabular}{|c|c|c|c|c|c|}
\hline COM energy $(\mathrm{TeV})$ & 3 & 0.4 & & 0.1 & \\
\hline$p$ energy $(\mathrm{GeV})$ & 16 & 16 & & 16 & \\
\hline$p$ 's/bunch & $2.5 \times 10^{13}$ & $2.5 \times 10^{13}$ & & $5 \times 10^{13}$ & \\
\hline Bunches/fill & 4 & 4 & & 2 & \\
\hline Rep. rate $(\mathrm{Hz})$ & 15 & 15 & & 15 & \\
\hline$p$ power (MW) & 4 & 4 & & 4 & \\
\hline$\mu /$ bunch & $2 \times 10^{12}$ & $2 \times 10^{12}$ & & $4 \times 10^{12}$ & \\
\hline$\mu$ power $(\mathrm{MW})$ & 28 & 4 & & 1 & \\
\hline Wall power (MW) & 204 & 120 & & 81 & \\
\hline Collider circum. (m) & 6000 & 1000 & & 350 & \\
\hline Ave bending field $(\mathrm{T})$ & 5.2 & 4.7 & & 3 & \\
\hline $\operatorname{rms} \Delta p / p(\%)$ & 0.16 & 0.14 & 0.12 & 0.01 & 0.003 \\
\hline $6 \mathrm{D} \epsilon_{6, N}(\pi \mathrm{m})^{3}$ & $1.7 \times 10^{-10}$ & $1.7 \times 10^{-10}$ & $1.7 \times 10^{-10}$ & $1.7 \times 10^{-10}$ & $1.7 \times 10^{-10}$ \\
\hline $\operatorname{rms} \epsilon_{n}(\pi \mathrm{mm} \mathrm{mrad})$ & 50 & 50 & 85 & 195 & 290 \\
\hline$\beta^{*}(\mathrm{~cm})$ & 0.3 & 2.6 & 4.1 & 9.4 & 14.1 \\
\hline$\sigma_{z}(\mathrm{~cm})$ & 0.3 & 2.6 & 4.1 & 9.4 & 14.1 \\
\hline$\sigma_{r} \operatorname{spot}(\mu \mathrm{m})$ & 3.2 & 26 & 86 & 196 & 294 \\
\hline$\sigma_{\theta}$ IP $(\mathrm{mrad})$ & 1.1 & 1.0 & 2.1 & 2.1 & 2.1 \\
\hline Tune shift & 0.044 & 0.044 & 0.051 & 0.022 & 0.015 \\
\hline$n_{\text {turns }}($ effective $)$ & 785 & 700 & 450 & 450 & 450 \\
\hline Luminosity $\left(\mathrm{cm}^{-2} \mathrm{~s}^{-1}\right)$ & $7 \times 10^{34}$ & $10^{33}$ & $1.2 \times 10^{32}$ & $2.2 \times 10^{31}$ & $10^{31}$ \\
\hline Higgs/yr & & & $1.9 \times 10^{3}$ & $4 \times 10^{3}$ & $3.9 \times 10^{3}$ \\
\hline
\end{tabular}

particles. Initial calculations suggest that the 6D phase space occupied by the initial muon bunches can be reduced by a factor of $10^{5}-10^{6}$ before multiple Coulomb scattering and energy straggling limit further reduction. We reiterate that ionization cooling is uniquely suited to muons because of the absence of strong nuclear interactions and electromagnetic shower production for these particles at energies around $200 \mathrm{MeV} / c$.

Rapid acceleration to the collider beam energy is needed to avoid excessive particle loss from decay. It can be achieved initially in a linear accelerator, and later in recirculating linear accelerators, rapid-cycling synchrotron, or fixed-field-alternating-gradient (FFAG) accelerators. Positive and negative muon bunches are then injected in opposite directions into a collider storage ring and brought into collision at the interaction point. The bunches circulate and collide for many revolutions before decay has depleted the beam intensities to an uninteresting level. Useful luminosity can be delivered

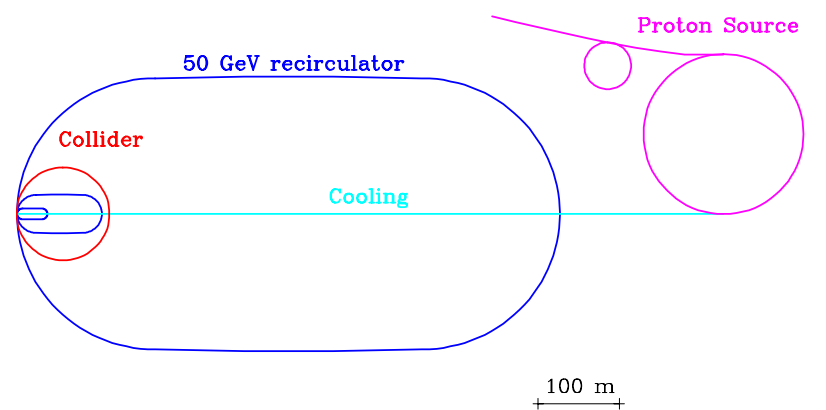

FIG. 2. (Color) Plan of a $0.1 \mathrm{TeV}$ COM muon collider. for about 800 revolutions for the high energy collider and 450 revolutions for the low energy collider.

There are many interesting and challenging problems that need to be resolved before the feasibility of building a muon collider can be demonstrated. For example, (i) heating from the very intense proton bunches may require the use of a liquid-jet target, and (ii) attaining the desired cooling factor in the ionization-cooling channel may require the development of rf cavities with thin beryllium windows operating at liquid-nitrogen temperatures in high solenoidal fields. In addition, the development of long

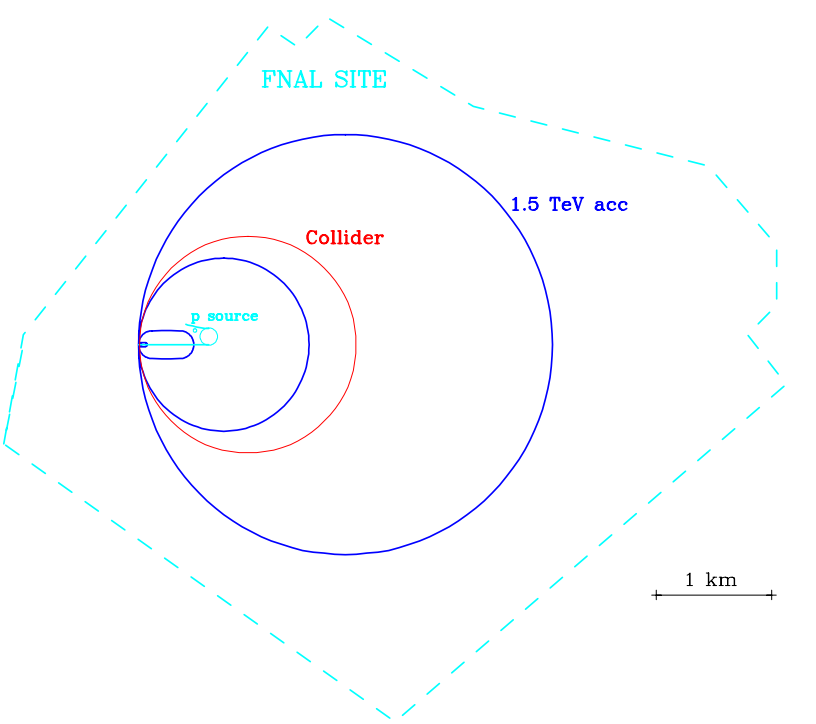

FIG. 3. (Color) Plan of a $3 \mathrm{TeV}$ COM muon collider shown on the Fermi National Laboratory site as an example. 
liquid-lithium lenses may be desirable to provide stronger radial focusing for the final cooling stages.

This article describes the status of our muon collider feasibility studies and is organized as follows. Section II gives a brief summary of the physics potential of muon colliders, including physics at the accelerator complex required for a muon collider. Section III describes the proton-driver specifications for a muon collider, and two site-dependent examples that have been studied in some detail. Section IV presents pion production, capture, and the pion-decay channel, and Sec. V discusses the design of the ionization-cooling channel needed to produce an intense muon beam suitable for acceleration and injection into the final collider. Sections VI and VII describe the acceleration scenario and collider ring, respectively. Section VIII discusses backgrounds at the collider interaction point, and Sec. IX deals with possible detector scenarios. A summary of the conclusions is given in Sec. $\mathrm{X}$

\section{THE PHYSICS POTENTIAL OF MUON COLLIDERS}

\section{A. Brief overview}

The physics agenda at a muon collider falls into three categories: FMC physics at a machine with centerof-mass energies of 100-500 GeV, next muon collider (NMC) physics at 3-4 TeV center-of-mass energies, and front-end physics with a high-intensity muon source.

The FMC will be a unique facility for neutral Higgs boson (or techniresonance) studies through $s$-channel resonance production. Measurements can also be made of the threshold cross sections for production of $W^{+} W^{-}, t \bar{t}$, $Z h$, and pairs of supersymmetry particles $-\chi_{1}^{+} \chi_{1}^{-}, \chi_{2}^{0} \chi_{1}^{0}$, $\tilde{\ell}^{+} \tilde{\ell}^{-}$, and $\tilde{\nu} \overline{\tilde{\nu}}$ - that will determine the corresponding masses to high precision. A $\mu^{+} \mu-\rightarrow Z^{0}$ factory, utilizing the partial polarization of the muons, could allow significant improvements in $\sin ^{2} \theta_{\mathrm{w}}$ precision and in $B$-mixing and $C P$-violating studies. In Fig. 4, we show the cross sections for standard model (SM) processes versus the COM energy at the FMC. For the unique $s$-channel Higgs boson production, where $\sqrt{s_{\mu \mu}}=m_{H}$, results for three different beam energy resolutions are presented.

The NMC will be particularly valuable for reconstructing supersymmetric particles of high mass from their complex cascade decay chains. Also, any $Z^{\prime}$ resonances within the kinematic reach of the machine would give enormous event rates. The effects of virtual $Z^{\prime}$ states would be detectable to high mass. If no Higgs bosons exist below $\sim 1 \mathrm{TeV}$, then the NMC would be the ideal machine for the study of strong $W W$ scattering at $\mathrm{TeV}$ energies.

At the front end, a high-intensity muon source will permit searches for rare muon processes sensitive to branching ratios that are orders of magnitude below present upper limits. Also, a high-energy muon-proton

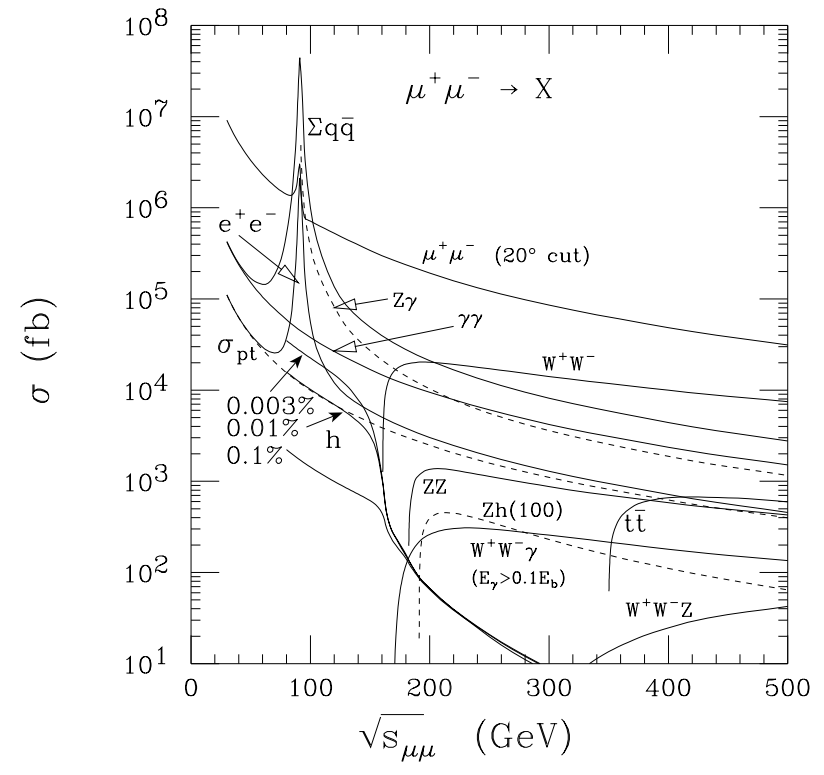

FIG. 4. Cross sections for SM processes vs the COM energy at the FMC. $\sigma_{p t} \equiv \sigma\left(\mu^{+} \mu^{-} \rightarrow \gamma^{*} \rightarrow e^{+} e^{-}\right)$. For the $s$-channel Higgs boson production, three different beam energy resolutions of $0.003 \%, 0.01 \%$, and $0.1 \%$ are presented.

collider can be constructed to probe high- $Q^{2}$ phenomena beyond the reach of the HERA $e p$ collider. In addition, the decaying muons will provide high-intensity neutrino beams for precision neutrino cross section measurements and for long-baseline experiments [57-66]. Plus, there are numerous other new physics possibilities for muon facilities $[44,39]$ that we will not discuss in detail in this document.

\section{B. Higgs boson physics}

The expectation that there will be a light (mass below $2 M_{W}$ ) SM-like Higgs boson provides a major motivation for the FMC, since such a Higgs boson can be produced with a very high rate directly in the $s$ channel. Theoretically, the lightest Higgs boson $h^{0}$ of the most general supersymmetric model is predicted to have mass below $150 \mathrm{GeV}$ and to be very SM-like in the usual decoupling limit. Indeed, in the minimal supersymmetric model, which contains the five Higgs bosons $h^{0}, H^{0}, A^{0}, H^{ \pm}$, one finds $m_{h^{0}} \lesssim 130 \mathrm{GeV}$ and the $h^{0}$ is SM-like if $m_{A^{0}} \gtrsim 130 \mathrm{GeV}$. Experimentally, global analyses of precision electroweak data now indicate a strong preference for a light SM-like Higgs boson. The goals of the FMC for studying the supersymmetry (SUSY) Higgs sector via $s$-channel resonance production are to measure the light Higgs mass, width, and branching fractions with high precision, in particular, sufficient to differentiate the minimal supersymmetric standard model (MSSM) $h^{0}$ from the SM $h_{\mathrm{SM}}$, and to find and study the heavier neutral Higgs bosons $H^{0}$ and $A^{0}$. 
The production of Higgs bosons in the $s$ channel with interesting rates is a unique feature of a muon collider $[45,67]$. The resonance cross section is

$$
\sigma_{h}(\sqrt{s})=\frac{4 \pi \Gamma(h \rightarrow \mu \bar{\mu}) \Gamma(h \rightarrow X)}{\left(s-m_{h}^{2}\right)^{2}+m_{h}^{2}\left(\Gamma_{\text {tot }}^{h}\right)^{2}} .
$$

Gaussian beams with root-mean-square (rms) energy resolution down to $R=0.003 \%$ are realizable. The corresponding rms spread $\sigma_{\sqrt{s}}$ in COM energy is

$$
\sigma_{\sqrt{s}}=(2 \mathrm{MeV})\left(\frac{R}{0.003 \%}\right)\left(\frac{\sqrt{s}}{100 \mathrm{GeV}}\right) .
$$

The effective $s$-channel Higgs cross section convolved with a Gaussian spread,

$$
\begin{aligned}
\bar{\sigma}_{h}(\sqrt{s})= & \frac{1}{\sqrt{2 \pi} \sigma_{\sqrt{s}}} \\
& \times \int \sigma_{h}(\sqrt{\hat{s}}) \exp \left[\frac{-(\sqrt{\hat{s}}-\sqrt{s})^{2}}{2 \sigma_{\sqrt{s}}^{2}}\right] d \sqrt{\hat{s}},
\end{aligned}
$$

is illustrated in Fig. 5 for $m_{h}=110 \mathrm{GeV}, \quad \Gamma_{h}=$ $2.5 \mathrm{MeV}$, and resolutions $R=0.01 \%, 0.06 \%$, and $0.1 \%$. A resolution $\sigma_{\sqrt{s}} \sim \Gamma_{h}$ is needed to be sensitive to the Higgs width. The light Higgs width is predicted to be

$$
\begin{aligned}
& \Gamma \approx 2-3 \mathrm{MeV} \text { if } \tan \beta \sim 1.8, \\
& \Gamma \approx 2-800 \mathrm{MeV} \text { if } \tan \beta \sim 20,
\end{aligned}
$$

for $80 \lesssim m_{h} \lesssim 120 \mathrm{GeV}$, where the smaller values apply in the decoupling limit of large $m_{A^{0}}$. We note that, in the MSSM, $m_{A^{0}}$ is required to be in the decoupling regime in the context of minimal super gravity (mSUGRA) boundary conditions in order that correct electroweak symmetry breaking arises after evolution of parameters from the unification scale. In particular, decoupling applies in mSUGRA at $\tan \beta \sim 1.8$, corresponding to the infrared fixed point of the top quark Yukawa coupling.



FIG. 5. Effective $s$-channel Higgs cross section $\bar{\sigma}_{h}$ obtained by convoluting the Breit-Wigner resonance formula with a Gaussian distribution for resolution $R$. From Ref. [45].
At $\sqrt{s}=m_{h}$, the effective $s$-channel Higgs cross section is

$$
\bar{\sigma}_{h} \simeq \frac{4 \pi}{m_{h}^{2}} \frac{B_{F}(h \rightarrow \mu \bar{\mu}) B_{F}(h \rightarrow X)}{\left[1+\frac{8}{\pi}\left(\frac{\sigma_{\sqrt{s}}}{\Gamma_{\text {tot }}^{2}}\right)^{2}\right]^{1 / 2}} .
$$

$B_{F}$ denotes the branching fraction for $h$ decay; also, note that $\bar{\sigma}_{h} \propto 1 / \sigma_{\sqrt{s}}$ for $\sigma_{\sqrt{s}}>\Gamma_{\text {tot }}^{h}$. At $\sqrt{s}=m_{h} \approx$ $110 \mathrm{Gev}$, the $b \bar{b}$ rates are

$$
\begin{gathered}
\text { signal } \approx 10^{4} \text { events } \times L\left(\mathrm{fb}^{-1}\right), \\
\text { background } \approx 10^{4} \text { events } \times L\left(\mathrm{fb}^{-1}\right),
\end{gathered}
$$

assuming a $b$-tagging efficiency $\epsilon \sim 0.5$ and an energy resolution of $0.003 \%$. The effective on-resonance cross sections for other $m_{h}$ values and other channels $\left(Z Z^{*}, W W^{*}\right)$ are shown in Fig. 6 for the SM Higgs. The rates for the MSSM Higgs are nearly the same as the SM rates in the decoupling regime of large $m_{A^{0}}$.

The important factors that make $s$-channel Higgs physics studies possible at a muon collider are energy resolutions $\sigma_{\sqrt{s}}$ of order a few $\mathrm{MeV}$, little bremsstrahlung and no beamstrahlung smearing, and precise tuning of the beam energy to an accuracy $\Delta E \sim 10^{-6} E$ through continuous spin-rotation measurements [12]. As a case study, we consider a SM-like Higgs boson with $m_{h} \approx 110 \mathrm{GeV}$. Prior Higgs discovery is assumed at the Tevatron (in $W h, t \bar{t} h$ production with $h \rightarrow b \bar{b}$ decay) or at the LHC (in $g g \rightarrow h$ production with $h \rightarrow \gamma \gamma, 4 \ell$ decays with a mass measurement of $\Delta m_{h} \sim 100 \mathrm{MeV}$ for an integrated luminosity of $L=300 \mathrm{fb}^{-1}$ ) or possibly at a next linear collider (NLC) (in $Z^{*} \rightarrow Z h, h \rightarrow b \bar{b}$ giving $\Delta m_{h} \sim 50 \mathrm{MeV}$ for $L=200 \mathrm{fb}^{-1}$ ). A muon collider ring design would be optimized to run at energy $\sqrt{s}=m_{h}$. For an initial Higgs-mass uncertainty of $\Delta m_{h} \sim 100 \mathrm{MeV}$, the maximum number of scan points required to locate the $s$-channel resonance peak at the muon collider is

$$
n=\frac{2 \Delta m_{h}}{\sigma_{\sqrt{s}}} \approx 100
$$

for a $R=0.003 \%$ resolution of $\sigma_{\sqrt{s}} \approx 2 \mathrm{MeV}$. The necessary luminosity per scan point $\left(L_{\mathrm{sp}}\right)$ to observe or eliminate the $h$ resonance at a significance level of $S / \sqrt{B}=3$ is $L_{\mathrm{sp}} \sim 1.5 \times 10^{-3} \mathrm{fb}^{-1}$. (The scan luminosity requirements increase for $m_{h}$ closer to $M_{Z}$; at $m_{h} \sim M_{Z}$ the $L_{\mathrm{sp}}$ needed is a factor of 50 higher.) The total luminosity then needed to tune to a Higgs boson with $m_{h}=110 \mathrm{GeV}$ is $L_{\text {tot }}=0.15 \mathrm{fb}^{-1}$. If the machine delivers $1.5 \times 10^{31} \mathrm{~cm}^{-2} \mathrm{~s}^{-1}\left(0.15 \mathrm{fb}^{-1} / \mathrm{yr}\right)$, then one year of running would suffice to complete the scan and measure the Higgs mass to an accuracy $\Delta m_{h} \sim 1 \mathrm{MeV}$. Figure 7 illustrates a simulation of such a scan.

Once the $h$ mass is determined to $\sim 1 \mathrm{MeV}$, a three-point fine scan [45] can be made across the peak with higher luminosity, distributed with $L_{1}$ at the observed peak position in $\sqrt{s}$ and $2.5 L_{1}$ at the wings $\left(\sqrt{s}=\right.$ peak $\left.\pm 2 \sigma_{\sqrt{s}}\right) . \quad$ Then, with $\quad L_{\text {tot }}=0.4 \mathrm{fb}^{-1}$ 


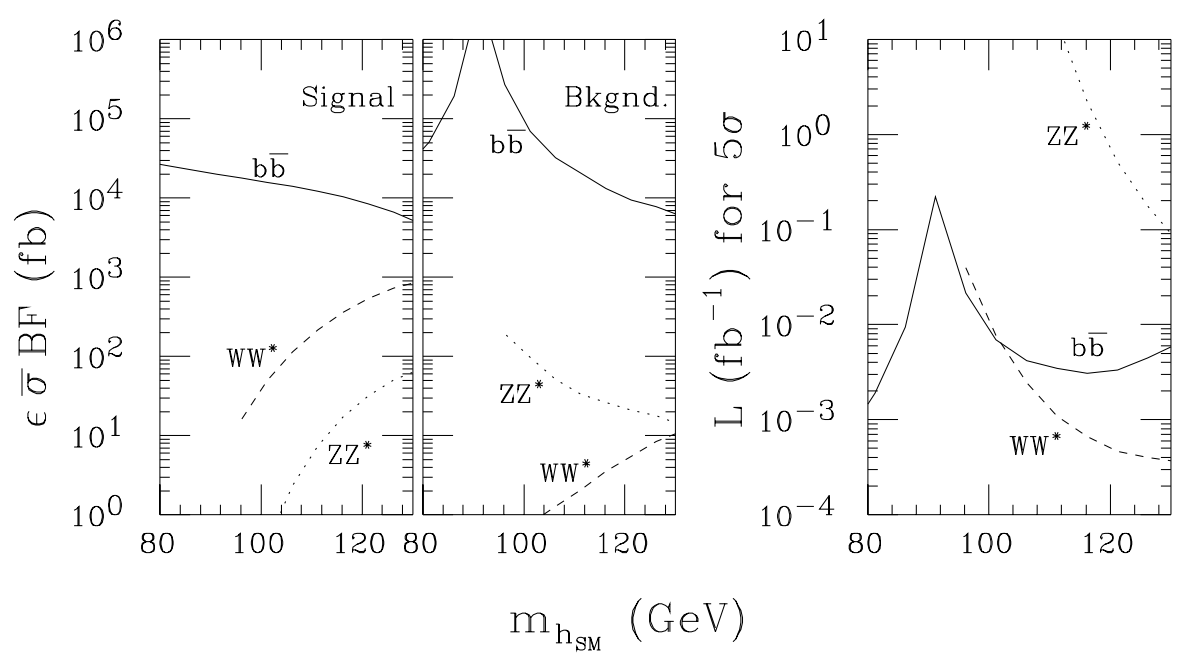

FIG. 6. The SM Higgs cross sections and backgrounds in $b \bar{b}, W W^{*}$, and $Z Z^{*}$. Also shown is the luminosity needed for a 5 standard deviation detection in $b \bar{b}$. From Ref. [45].

the following accuracies would be achievable: $16 \%$ for $\Gamma_{\text {tot }}^{h}, 1 \%$ for $\sigma B_{F}(b \bar{b})$, and $5 \%$ for $\sigma B_{F}\left(W W^{*}\right)$. The ratio $r=B_{F}\left(W W^{*}\right) / B_{F}(b \bar{b})$ is sensitive to $m_{A^{0}}$ for $m_{A^{0}}$ values below $500 \mathrm{GeV}$. For example, $r_{\mathrm{MSSM}} / r_{\mathrm{SM}}=$ $0.3,0.5,0.8$ for $m_{A^{0}}=200,250,400 \mathrm{GeV}$ [45]. Thus, using $s$-channel measurements of the $h$, it may be possible not only to distinguish the $h^{0}$ from the SM $h_{\mathrm{SM}}$ but also to infer $m_{A^{0}}$.

The study of the other neutral MSSM Higgs bosons at a muon collider via the $s$ channel is also of major interest. Finding the $H^{0}$ and $A^{0}$ may not be easy at other colliders. At the LHC the region $m_{A^{0}}>200 \mathrm{GeV}$ is deemed to be inaccessible for $3 \lesssim \tan \beta \lesssim 5-10$ [68]. At an NLC the $e^{+} e^{-} \rightarrow H^{0} A^{0}$ production process may be kinematically inaccessible if $H^{0}$ and $A^{0}$ are heavy (mass $>230 \mathrm{GeV}$ for $\sqrt{s}=500 \mathrm{GeV}$ ). At a $\gamma \gamma$ collider, very high luminosity $\left(\sim 200 \mathrm{fb}^{-1}\right)$ would be needed for $\gamma \gamma \rightarrow H^{0}, A^{0}$ studies.



FIG. 7. Number of events and statistical errors in the $b \bar{b}$ final states as a function of $\sqrt{s}$ in the vicinity of $m_{h_{\mathrm{SM}}}=110 \mathrm{GeV}$, assuming $R=0.003 \%$. From Ref. [45].
At a muon collider the resolution requirements for $s$-channel $H^{0}$ and $A^{0}$ studies are not as demanding as for the $h^{0}$ because the $H^{0}, A^{0}$ widths are broader; typically $\Gamma \sim 30 \mathrm{MeV}$ for $m_{A^{0}}<2 m_{t}$ and $\Gamma \sim 3 \mathrm{GeV}$ for $m_{A^{0}}>2 m_{t}$. Consequently, $R \sim 0.1 \%\left(\sigma_{\sqrt{s}} \sim 70 \mathrm{MeV}\right)$ is adequate for a scan. This is important, since higher instantaneous luminosities (corresponding to $L \sim 2-$ $10 \mathrm{fb}^{-1} / \mathrm{yr}$ ) are possible for $R \sim 0.1 \%$ (as contrasted with the $L \sim 0.15 \mathrm{fb}^{-1} / \mathrm{yr}$ for the much smaller $R \sim 0.003 \%$ preferred for studies of the $h^{0}$ ). A luminosity per scan point $L_{\mathrm{sp}} \sim 0.1 \mathrm{fb}^{-1}$ probes the parameter space with $\tan \beta>2$. The $\sqrt{s}$ range over which the scan should be made depends on other information available to indicate the $A^{0}$ and $H^{0}$ mass range of interest. A wide scan would not be necessary if $r$ is measured with the above-described precision to obtain an approximate value of $m_{A^{0}}$.

In the MSSM, $m_{A^{0}} \approx m_{H^{0}} \approx m_{H^{ \pm}}$at large $m_{A^{0}}$ (as expected for mSUGRA boundary conditions), with a very close degeneracy in these masses for large $\tan \beta$. In such a circumstance, only an $s$-channel scan with the good resolution possible at a muon collider may allow separation of the $A^{0}$ and $H^{0}$ states; see Fig 8.

\section{Light particles in technicolor models}

In most technicolor models, there will be light neutral and colorless technipion resonances, $\pi_{T}^{0}$ and $\pi_{T}^{0 \prime}$, with masses below $500 \mathrm{GeV}$. Sample models include the recent top-assisted technicolor models [69], in which the technipion masses are typically above $100 \mathrm{GeV}$, and models [70] in which the masses of the neutral colorless resonances come primarily from the one-loop effective potential and the lightest state typically has mass as low as $10-100 \mathrm{GeV}$. The widths of these light neutral and colorless states in the top-assisted models will be of order 0.1-50 GeV [71]. In the one-loop models, the width 


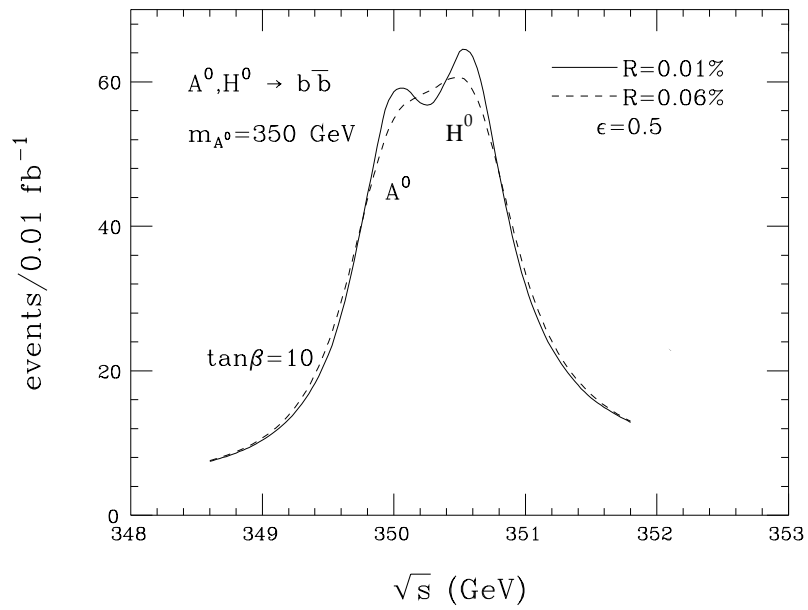

FIG. 8. Separation of $A^{0}$ and $H^{0}$ signals for $\tan \beta=10$. From Ref. [45].

of the lightest technipion is typically in the range 3$50 \mathrm{MeV}$. Neutral technirho and techniomega resonances are also a typical feature of technicolor models. In all models, these resonances are predicted to have substantial Yukawa-like couplings to muons and would be produced in the $s$ channel at a muon collider,

$$
\mu^{+} \mu^{-} \rightarrow \pi_{T}^{0}, \pi_{T}^{0 \prime}, \rho_{T}^{0}, \omega_{T}^{0},
$$

with high event rates. The peak cross sections for these processes are estimated to be $\approx 10^{4}-10^{7} \mathrm{fb}$ [71]. The dominant decay modes depend on eigenstate composition and other details but typically are [71]

$$
\begin{gathered}
\pi_{T}^{0} \rightarrow g g, b \bar{b}, \tau \bar{\tau}, c \bar{c}, t \bar{t}, \\
\pi_{T}^{0 \prime} \rightarrow g g, b \bar{b}, c \bar{c}, t \bar{t}, \tau^{+} \tau^{-}, \\
\rho_{T}^{0} \rightarrow \pi_{T} \pi_{T}, W \pi_{T}, W W, \\
\omega_{T}^{0} \rightarrow c \bar{c}, b \bar{b}, \tau \bar{\tau}, t \bar{t}, \gamma \pi_{T}^{0}, Z \pi_{T}^{0} .
\end{gathered}
$$

Such resonances would be easy to find and study at a muon collider.

\section{Exotic narrow resonance possibilities}

There are important types of exotic physics that would be best probed in $s$-channel production of a narrow resonance at a muon collider. Many extended Higgs sector models contain a doubly charged Higgs boson $\Delta^{--}$(and its $\Delta^{++}$partner) that couples to $\mu^{-} \mu^{-}$via a Majorana coupling. The $s$-channel process $\mu^{-} \mu^{-} \rightarrow$ $\Delta^{--}$has been shown [72] to probe extremely small values of this Majorana coupling, in particular, values naturally expected in models where such couplings are responsible for neutrino mass generation. In supersymmetry, it is possible that there is $R$-parity violation. If $R$-parity violation is of the purely leptonic type, the coupling $\lambda_{\mu \tau \mu}$ for $\mu^{-} \mu^{+} \rightarrow \tilde{\nu}_{\tau}$ is very possibly the largest such coupling and could be related to neutrino mass generation.
This coupling can be probed down to quite small values via $s$-channel $\tilde{\nu}_{\tau}$ production at the muon collider [73].

\section{E. $Z$ factory}

A muon collider operating at the $Z$-boson resonance energy is an interesting option for measurement of polarization asymmetries, $B_{s}^{0}-\bar{B}_{s}^{0}$ mixing, and of $C P$ violation in the $B$-meson system [74]. The muon collider advantages are the partial muon beam polarization and the long $B$-decay length for $B$ mesons produced at this $\sqrt{s}$. The left-right asymmetry $A_{L R}$ is the most accurate measure of $\sin ^{2} \theta_{\mathrm{w}}$, since the uncertainty is statistics dominated. The present polarization measurements from LEP and from the SLD Collaboration at SLC show deviations from the standard model prediction by $2.4 \sigma$ in $A_{L R}^{0}, 1.9 \sigma$ in $A_{F B}^{0, b}$, and $1.7 \sigma$ in $A_{F B}^{0, \tau}$ [75]. The $C P$ angle $\beta$ could be measured from $B^{0} \rightarrow K_{s} J / \psi$ decays. To achieve significant improvements over existing measurements and those at future $B$ facilities, a data sample of $10^{8} \mathrm{Z}$-boson events/yr would be needed. This corresponds to a luminosity $>0.15 \mathrm{fb}^{-1} / \mathrm{yr}$, which is well within the domain of muon collider expectations; $R \sim$ $0.1 \%$ would be more than adequate, given the substantial $\sim 2.4 \mathrm{GeV}$ width of the $Z$.

\section{F. Threshold measurements at a muon collider}

With $10 \mathrm{fb}^{-1}$ integrated luminosity devoted to a measurement of a threshold cross section, the following precisions on particle masses may be achievable [76]:

$$
\begin{aligned}
& \mu^{+} \mu^{-} \rightarrow W^{+} W^{-} \quad \Delta M_{W}=20 \mathrm{MeV}, \\
& \mu^{+} \mu^{-} \rightarrow t \bar{t} \quad \Delta m_{t}=0.2 \mathrm{GeV}, \\
& \mu^{+} \mu^{-} \rightarrow Z h \quad \Delta m_{h}=140 \mathrm{MeV}
\end{aligned}
$$

(if $m_{h}=100 \mathrm{GeV}$ ). Precision $M_{W}$ and $m_{t}$ measurements allow important tests of electroweak radiative corrections through the relation

$$
M_{W}=M_{Z}\left[1-\frac{\pi \alpha}{\sqrt{2} G_{\mu} M_{W}^{2}(1-\delta r)}\right]^{1 / 2},
$$

where $\delta r$ represents loop corrections. In the SM, $\delta r$ depends on $m_{t}^{2}$ and $\log m_{h}$. The optimal precision for tests of this relation is $\Delta M_{W} \approx \frac{1}{140} \Delta m_{t}$, so the uncertainty on $M_{W}$ is the most critical. With $\Delta M_{W}=20 \mathrm{MeV}$, the SM Higgs mass could be inferred to an accuracy

$$
\Delta m_{h_{\mathrm{SM}}}=30 \mathrm{GeV} \times\left(\frac{m_{h}}{100 \mathrm{GeV}}\right) .
$$

Alternatively, once $m_{h}$ is known from direct measurements, SUSY loop contributions can be tested.

In top-quark production at a muon collider above the threshold region, modest muon polarization would allow sensitive tests of anomalous top quark couplings [77].

One of the important physics opportunities for the first muon collider is the production of the lighter chargino, 
$\tilde{\chi}_{1}^{+}$[78]. Fine-tuning arguments in mSUGRA suggest that it should be lighter than $200 \mathrm{GeV}$. A search at the upgraded Tevatron for the process $q \bar{q} \rightarrow \tilde{\chi}_{1}^{+} \bar{\chi}_{2}^{0}$ with $\tilde{\chi}_{1}^{+} \rightarrow \tilde{\chi}_{1}^{0} \ell^{+} \nu$ and $\tilde{\chi}_{2}^{0} \rightarrow \tilde{\chi}_{1}^{0} \ell^{+} \ell^{-}$decays can potentially reach masses $m_{\tilde{\chi}_{1}^{+}} \simeq m_{\tilde{\chi}_{2}^{0}} \sim 170 \mathrm{GeV}$ with $2 \mathrm{fb}^{-1}$ luminosity and $\sim 230 \mathrm{GeV}$ with $10 \mathrm{fb}^{-1}$ [79]. The mass difference $M\left(\tilde{\chi}_{2}^{0}\right)-M\left(\tilde{\chi}_{1}^{0}\right)$ can be determined from the $\ell^{+} \ell^{-}$mass distribution.

The two contributing diagrams in the chargino pair production process are shown in Fig. 9; the two amplitudes interfere destructively. The $\tilde{\chi}_{1}^{+}$and $\tilde{\nu}_{\mu}$ masses can be inferred from the shape of the cross section in the threshold region [80]. The chargino decay is $\tilde{\chi}_{1}^{+} \rightarrow f \bar{f}^{\prime} \tilde{\chi}_{1}^{0}$. Selective cuts suppress the background from $W^{+} W^{-}$production and leave $\sim 5 \%$ signal efficiency for 4 jets $+\not_{T}$ events. Measurements at two energies in the threshold region with total luminosity $L=50 \mathrm{fb}$ and resolution $R=0.1 \%$ can give the accuracies listed in Table II on the chargino mass for the specified values of $m_{\tilde{\chi}_{1}^{+}}$ and $m_{\tilde{\nu}_{\mu}}$.

\section{G. Heavy particles of supersymmetry}

The requirements of gauge coupling unification can be used to predict the mean SUSY mass scale, given the value of the strong coupling constant at the $Z$-mass scale. Figure 10 shows the SUSY GUT predictions versus $\alpha_{s}\left(M_{Z}\right)$. For the value $\alpha_{s}\left(M_{Z}\right)=0.1214 \pm 0.0031$ from a new global fit to precision electroweak data [75], a mean SUSY mass of order $1 \mathrm{TeV}$ is expected. Thus, it is likely that some SUSY particles will have masses at the TeV scale. Large masses for the squarks of the first family are perhaps the most likely in that this would provide a simple cure for possible flavor changing neutral current difficulties.

At the LHC, mainly squarks and gluinos will be produced; these decay to lighter SUSY particles. The LHC will be a great SUSY machine, but some sparticle measurements will be very difficult or impossible there [81,82], namely, (i) the determination of the LSP mass (LHC measurements give SUSY mass differences), (ii) study of sleptons of mass $\gtrsim 200 \mathrm{GeV}$ because DrellYan production becomes too small at these masses, (iii) study of heavy gauginos $\tilde{\chi}_{2}^{ \pm}$and $\tilde{\chi}_{3,4}^{0}$, which are mainly Higgsino and have small direct production rates

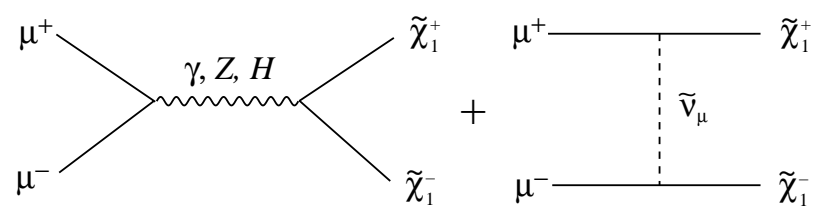

FIG. 9. Diagrams for production of the lighter chargino.
TABLE II. Achievable uncertainties with $50 \mathrm{fb}^{-1}$ luminosity on the mass of the lighter chargino for representative $m_{\tilde{\chi}_{1}^{+}}$and $m_{\tilde{\nu}_{\mu}}$ masses. From Ref. [80].

\begin{tabular}{ccc}
\hline \hline$\Delta m_{\tilde{\chi}_{1}^{+}}(\mathrm{MeV})$ & $m_{\tilde{\chi}_{1}^{+}}(\mathrm{GeV})$ & $m_{\tilde{\nu}_{\mu}}(\mathrm{GeV})$ \\
\hline 35 & 100 & 500 \\
45 & 100 & 300 \\
150 & 200 & 500 \\
300 & 200 & 300 \\
\hline \hline
\end{tabular}

and small branching fractions to channels usable for detection, and (iv) study of heavy Higgs bosons $H^{ \pm}, H^{0}, A^{0}$ when the MSSM $\tan \beta$ parameter is not large and their masses are larger than $2 m_{t}$, so that cross sections are small and decays to $t \bar{t}$ are likely to be dominant (their detection is deemed impossible if SUSY decays dominate).

Detection and study of the many scalar particles predicted in supersymmetric models could be a particularly valuable contribution of a high energy lepton collider. However, since pair production of scalar particles at a lepton collider is $P$-wave suppressed, energies well above threshold are needed for sufficient production rates; see Fig. 11. For scalar particle masses of order $1 \mathrm{TeV}$, a collider energy of $3-4 \mathrm{TeV}$ is needed to get past the threshold suppression. A muon collider operating in this energy range with high luminosity $\left(L \sim 10^{2}-10^{3} \mathrm{fb}^{-1} / \mathrm{yr}\right.$ ) would provide sufficient event rates to reconstruct heavy sparticles from their complex cascade decay chains $[82,84]$.

In string models, it is very natural to have extra $Z$ bosons in addition to low energy supersymmetry. The $s$-channel production of a $Z^{\prime}$ boson at the resonance energy would give enormous event rates at the NMC. Moreover, the $s$-channel contributions of $Z^{\prime}$ bosons with mass far above the kinematic reach of the collider could be revealed as contact interactions [85].

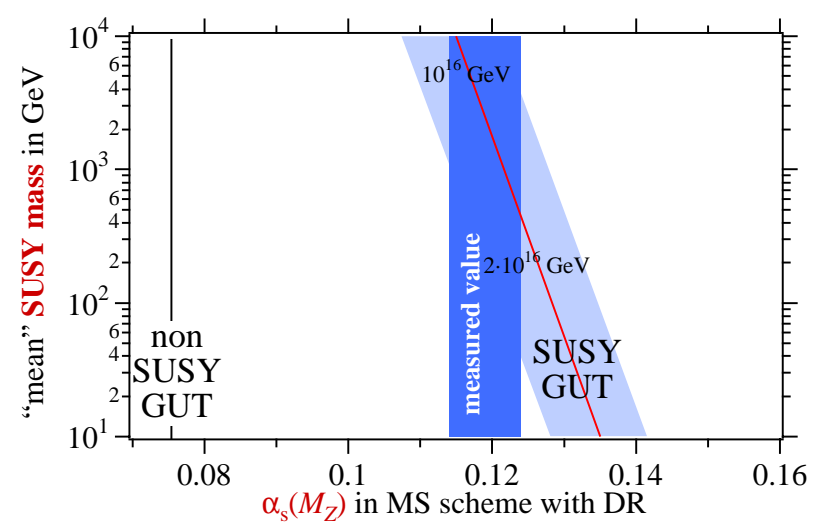

FIG. 10. (Color) $\alpha_{s}$ prediction in supersymmetric GUT with minimal particle content in the dimensional regularization scheme. 

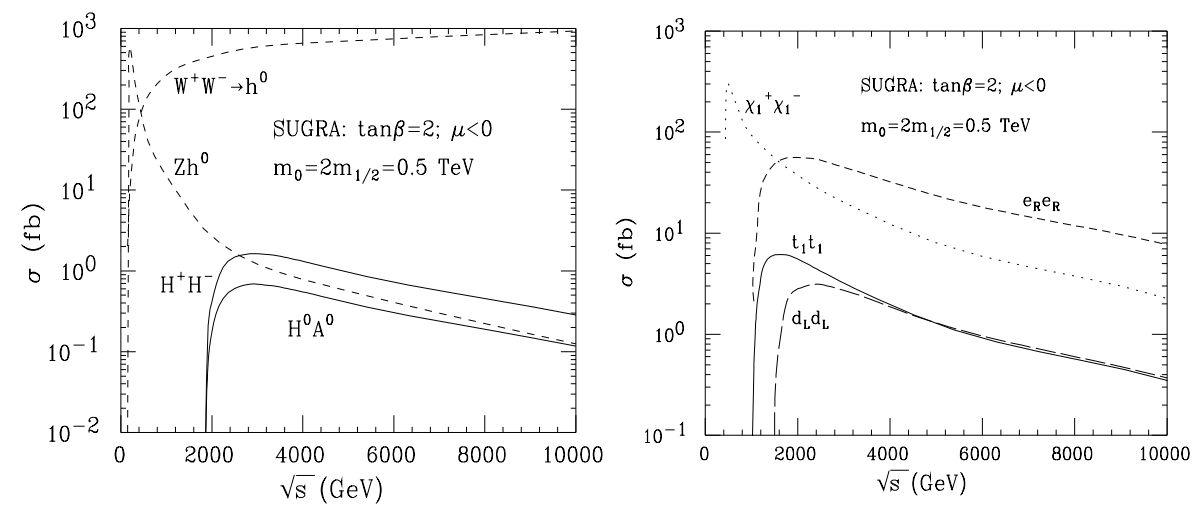

FIG. 11. Cross sections for pair production of Higgs bosons and scalar particles at a high energy muon collider. From Ref. [83].

\section{H. Strong scattering of weak bosons}

The scattering of weak bosons can be studied at a high energy muon collider through the process in Fig. 12. The amplitude for the scattering of longitudinally polarized $W$ bosons behaves like

$$
A\left(W_{L} W_{L} \rightarrow W_{L} W_{L}\right) \sim m_{H}^{2} / v^{2},
$$

if there is a light Higgs boson, and like

$$
A\left(W_{L} W_{L} \rightarrow W_{L} W_{L}\right) \sim s_{W W} / v^{2},
$$

if no light Higgs boson exists; here, $s_{W W}$ is the square of the $W W \mathrm{COM}$ energy and $v=246 \mathrm{GeV}$. In the latter scenario, partial-wave unitarity of $W_{L} W_{L} \rightarrow W_{L} W_{L}$ requires that the scattering of weak bosons becomes strong at energy scales of order 1-2 TeV. Thus, subprocess energies $\sqrt{s_{W W}} \geq 1.5 \mathrm{TeV}$ are needed to probe strong $W W$ scattering effects.

The nature of the dynamics in the $W W$ sector is unknown. Models for this scattering assume heavy resonant particles (isospin scalar and vector) or a nonresonant amplitude based on a unitarized extrapolation of the low energy theorem behavior $A \sim s_{W W} / v^{2}$. In all models, impressive signals of strong $W W$ scattering are obtained at the NMC, with cross sections typically of order $50 \mathrm{fb}$ [86]. Event rates are such that the various weak-isospin channels $(I=0,1,2)$ could be studied in detail as a func-

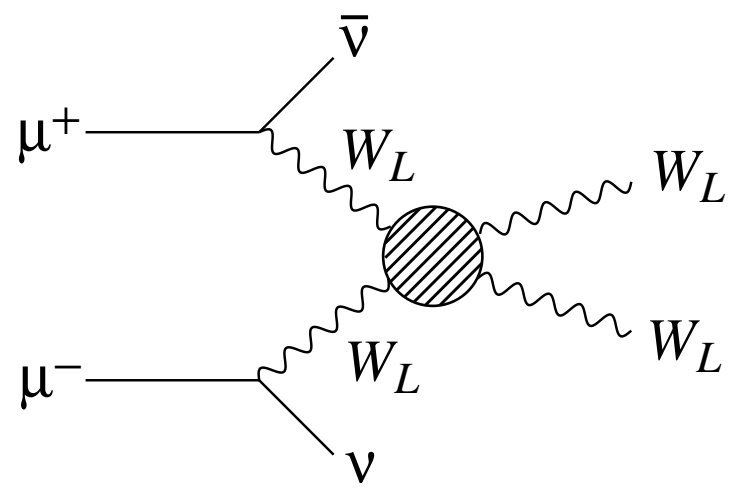

FIG. 12. Symbolic diagram for strong $W W$ scattering. tion of $s_{W W}$. After several years of operation, it would even be possible to perform such a study after projecting out the different final polarization states $\left(W_{L} W_{L}, W_{L} W_{T}\right.$, and $W_{T} W_{T}$ ), thereby enabling one to verify that it is the $W_{L} W_{L}$ channel in which the strong scattering is taking place.

\section{Front end physics}

New physics is likely to have important lepton flavor dependence and may be most apparent for heavier flavors. The intense muon source available at the front end of the muon collider will provide many opportunities for uncovering such physics.

\section{Rare muon decays}

The planned muon flux of $\sim 10^{14}$ muons/sec for a muon collider dramatically eclipses the flux, $\sim 10^{8}$ muons $/ \mathrm{sec}$, of present sources. With an intense source, the rare muon processes $\mu \rightarrow e \gamma$ (for which the current branching fraction limit is $\left.0.49 \times 10^{-12}\right), \mu N \rightarrow e N$ conversion, and the muon electric dipole moment can be probed at very interesting levels. A generic prediction of supersymmetric grand unified theories is that these lepton flavor violating or $C P$-violating processes should occur via loops at significant rates, e.g., $B_{F}(\mu \rightarrow e \gamma) \sim 10^{-13}$. Lepton-flavor violation can also occur via $Z^{\prime}$ bosons, leptoquarks, and heavy neutrinos [87].

\section{Neutrino flux}

The decay of a muon beam leads to neutrino beams of well-defined flavors. A muon collider would yield a neutrino flux 1000 times that presently available [88]. This would result in $\sim 10^{6} \nu N$ and $\bar{\nu} N$ events per year, which could be used to measure charm production $(\sim 6 \%$ of the total cross section) and measure $\sin ^{2} \theta_{W}$ (and infer the $W$ mass to an accuracy $\Delta M_{W} \simeq 30-50 \mathrm{MeV}$ in one year) [57-65]. 


\section{Neutrino oscillations}

A special purpose muon ring has been proposed [58] to store $\sim 10^{21} \mu^{+}$or $\mu^{-}$per year and obtain $\sim 10^{20}$ neutrinos per year from muon decays along $\sim 75 \mathrm{~m}$ straight sections of the ring, which would be pointed toward a distant neutrino detector. The neutrino fluxes from $\mu^{-} \rightarrow \nu_{\mu} \bar{\nu}_{e} e^{-}$or from $\mu^{+} \rightarrow \bar{\nu}_{\mu} \nu_{e} e^{+}$ decays can be calculated with little systematic error. Then, for example, from the decays of stored $\mu^{--}$s, the following neutrino oscillation channels could be studied by detection of the charged leptons from the interactions of neutrinos in the detector:

$\begin{array}{cc}\text { oscillation } & \text { detect } \\ \nu_{\mu} \rightarrow \nu_{e} & e^{-} \\ \nu_{\mu} \rightarrow \nu_{\tau} & \tau^{-} \\ \bar{\nu}_{e} \rightarrow \bar{\nu}_{\mu} & \mu^{+} \\ \bar{\nu}_{e} \rightarrow \bar{\nu}_{\tau} & \tau^{+}\end{array}$

The detected $e^{-}$or $\mu^{+}$have the "wrong sign" from the leptons produced by the interactions of the $\bar{\nu}_{e}$ and $\nu_{\mu}$ flux. The known neutrino fluxes from muon decays could be used for long-baseline oscillation experiments at any detector on Earth. The probabilities for vacuum oscillations between two neutrino flavors are given by

$$
P\left(\nu_{a} \rightarrow \nu_{b}\right)=\sin ^{2} 2 \theta \sin ^{2}\left(1.27 \delta m^{2} L / E\right),
$$

with $\delta m^{2}$ in $\mathrm{eV}^{2}$ and $L / E$ in $\mathrm{km} / \mathrm{GeV}$. In a very long baseline experiment from Fermilab to the Gran Sasso laboratory or the Kamioka mine $\left[L=\mathcal{O}\left(10^{4}\right) \mathrm{km}\right]$ with $\nu$ energies $E_{\nu}=20-50 \mathrm{GeV}(L / E=500-200 \mathrm{~km} / \mathrm{GeV})$, neutrino charged-current interaction rates of $\sim 10^{3} / \mathrm{yr}$ would result. In a long baseline experiment from Fermilab to the Soudan mine $(L=732 \mathrm{~km})$, the corresponding interaction rate is $\sim 10^{4} / \mathrm{yr}$. Such an experiment would have sensitivity to oscillations down to $\delta m^{2} \sim$ $10^{-4}-10^{-5} \mathrm{eV}^{2}$ for $\sin ^{2} 2 \theta=1$ [58].

\section{4. $\mu$ p collider}

The possibility of colliding $200 \mathrm{GeV}$ muons with $1000 \mathrm{GeV}$ protons at Fermilab is under study. This collider would reach a maximum $Q^{2} \sim 8 \times 10^{5} \mathrm{GeV}^{2}$, which is $\sim 8$ times the reach of the HERA $e p$ collider, and deliver a luminosity $\sim 10^{33} \mathrm{~cm}^{-2} \mathrm{~s}^{-1}$, which is $\sim 300$ times the HERA luminosity. The $\mu p$ collider would produce $\sim 10^{6}$ neutral-current deep-inelastic-scattering events per year at $Q^{2}>5000 \mathrm{GeV}^{2}$, which is more than a factor of $10^{3}$ higher than at HERA. In the new physics realm, leptoquark couplings and contact interactions, if present, are likely to be larger for muons than for electrons. This $\mu p$ collider would have sufficient sensitivity to probe leptoquarks up to a mass $M_{L Q} \sim 800 \mathrm{GeV}$ and contact interactions to a scale $\Lambda \sim 6-9 \mathrm{TeV}$ [89].

\section{J. Summary of the physics potential}

The first muon collider offers unique probes of supersymmetry (particularly $s$-channel Higgs boson reso- nances) and technicolor models (via $s$-channel production of techniresonances), high-precision threshold measurements of $W, t$ and SUSY particle masses, tests of SUSY radiative corrections that indirectly probe the existence of high-mass squarks, and a possible $Z^{0}$ factory for improved precision in polarization measurements and for $B$-physics studies of $C P$ violation and mixing.

The "next muon collider" guarantees access to heavy SUSY scalar particles and $Z^{\prime}$ states or to strong $W W$ scattering if there are no Higgs bosons and no supersymmetry.

The front end of a muon collider offers dramatic improvements in sensitivity for flavor-violating transitions (e.g., $\mu \rightarrow e \gamma$ ), access to high- $Q^{2}$ phenomena in deepinelastic muon-proton and neutrino-proton interactions, and the ability to probe very small $\delta \mathrm{m}^{2}$ via neutrinooscillation studies in long-baseline experiments.

The muon collider would be crucial to unraveling the flavor dependence of any type of new physics that is found at the next generation of colliders.

Thus, muon colliders are robust options for probing new physics that may not be accessible at other colliders.

\section{PROTON DRIVER}

The overview of the required parameters is followed by a description of designs that have been studied in some detail. The section concludes with a discussion of the outstanding open issues.

\section{A. Specifications}

The proton driver requirements are determined by the design luminosity of the collider and the efficiencies of muon collection, cooling, transport, and acceleration. The baseline specification is for a $4 \mathrm{MW}, 16 \mathrm{GeV}$ or a $7 \mathrm{MW}$, $30 \mathrm{GeV}$ proton driver, with a repetition rate of $15 \mathrm{~Hz}$ and $10^{14}$ protons per cycle in two bunches (for the $100 \mathrm{GeV}$ machine) or four bunches (for the higher energies) of $5 \times 10^{13}$ or $2.5 \times 10^{13}$ protons, respectively. Half of the bunches are used to make $\mu^{-}$and the rest for $\mu^{+}$[90].

The total beam power is several MW, which is larger than that of existing synchrotrons. However, except for bunch length, these parameters are similar to those of Kaon factories [91] and spallation neutron sources [92]. As in those cases, the proton driver must have very low losses to permit inexpensive maintenance of components.

The rms bunch length for the protons on target has to be about $1 \mathrm{~ns}$ in order to (i) reduce the initial longitudinal emittance of muons entering the cooling system and (ii) optimize the production of polarized muons. Although bunches of up to $6 \times 10^{13}$ protons per cycle have been accelerated, the required peak current is $2000 \mathrm{~A}$, which is unprecedented.

Since the collection of highly polarized $\mu$ 's is inefficient (see Sec. IVG), the proton driver should eventually provide an additional factor of 2 or more in proton 
intensity to permit the luminosity to be maintained for polarized muon beams.

\section{B. Possible options}

Accelerator designs are site, and to some extent, time dependent, and there have been three studies at three different energies (30 GeV [93], $16 \mathrm{GeV}$ [94], and $24 \mathrm{GeV}$ [95-98]; see also [99]). In general, if the final energy is higher, the required currents are lower, bunch manipulation and apertures are easier, and the final momentum spread and space-charge tune shifts are less. Lowering the final energy gives somewhat more $\pi$ 's/W, a lower $\mathrm{rf}$ requirement $\left(V_{\mathrm{rf}} \sim E^{2}\right)$, and perhaps a lower facility cost.

In the low energy muon collider, where two bunches of protons of $5 \times 10^{13}$ are required on target, two bunches can be merged outside the driver. These two bunches would be extracted simultaneously from two different extraction ports and fed by different transmission lines to the same target. By arranging the path lengths of the two lines appropriately, the two bunches can be exactly merged.

\section{A generic design}

A 7 MW collider-driver design based on parameters originally proposed in the Snowmass Feasibility Study [93] consists of a $600 \mathrm{MeV}$ linac, a $3.6 \mathrm{GeV}$ booster, and a $30 \mathrm{GeV}$ driver. Both linac and booster are based on the BNL Spallation Neutron Source design [92], using a lower repetition rate and a lower total number of protons per pulse. For the four-bunch case $\left(2.5 \times 10^{13}\right.$ protons per bunch), the (95\%) bunch area is assumed to be $2 \mathrm{eV} \mathrm{s}$ at injection and $<4.5 \mathrm{eV} \mathrm{s}$ at extraction. The driver lattice is derived from the lattice of the Japan Hadron Facility (JHF) driver using $90^{\circ}$ FODO cells with missing dipoles in every third FODO cell, allowing a transition energy that is higher than the maximum energy or, perhaps, imaginary.

\section{FNAL study}

If a muon collider is built at an existing laboratory, then possibilities abound for symbiotic relationships with the other facilities and programs of that laboratory. For example, the proton driver for a muon collider might result from an upgrade of existing proton-source capabilities, and such an upgrade could then also enhance other future programs that use the proton beams.

Fermilab has conceived such a proton-source development plan [100] with three major components: an upgraded linac, a prebooster, and a new booster, with the two boosters being rapid-cycling $(15 \mathrm{~Hz})$ synchrotrons. The two synchrotrons operate in series; the four proton bunches for the muon collider are formed in the prebooster and then accelerated sequentially in the prebooster and the booster. The plan could be implemented in stages, and other pro- grams would benefit from each stage, but all three components are required to meet the luminosity goals of the muon colliders that have been considered so far.

Table III presents the major parameters of the two rings. Whenever the needs of the muon collider itself allow some flexibility, the parameters have been chosen to optimize the resulting facility as a proton source for the rest of the future program at Fermilab. For example, the machine circumferences and rf-harmonic numbers result in bunch trains that are compatible with the existing downstream proton machines.

A muon collider requires proton bunches that are both very intense and, at the pion-production target, very short. Strong transverse and longitudinal space-charge forces might disrupt such bunches in the synchrotrons unless measures to alleviate those effects are incorporated in the design. The Laslett incoherent-space-charge tune shift quantifies the severity of the transverse effects. A useful approximation for the space-charge tune shift $\Delta \nu_{s c}$ at the center of a round Gaussian beam is

$$
\Delta \nu_{s c}=-\frac{3 r_{p} N_{\mathrm{tot}}}{2 \epsilon_{n} \beta \gamma^{2} b} .
$$

In this expression, $r_{p}=1.535 \times 10^{-18} \mathrm{~m}$ is the so-called electromagnetic radius of the proton, $N_{\text {tot }}$ is the total number of protons in the ring, $\epsilon_{n}$ is the $95 \%$ normalized transverse emittance, $\beta$ and $\gamma$ are the usual Lorentz kinematical factors, and $b \leq 1$ is the bunching factor, defined as the ratio of the average beam current to the peak current.

The approximation (20) implies that for a given total number of protons, here $10^{14}$, the factors in the denominator are the only ways to reduce the tune shift to a specified maximum tolerable value, taken as 0.4 . The bunching factor can be raised somewhat by careful tailoring of beam distributions, but here a typical value of 0.25 is conservatively assumed. Achieving the desired beam intensity then requires a combination of high injection energy, here taken as $1 \mathrm{GeV}$ into the first ring, and large transverse normalized emittances, here assumed to be about $200 \pi \mathrm{mm}$ mrad. The corresponding required aperture is about $13 \mathrm{~cm}$ in the first ring and about $10 \mathrm{~cm}$ in the

TABLE III. Baseline proton-driver parameters of the FNAL study.

\begin{tabular}{lccc}
\hline \hline & Linac & Booster & Driver \\
\hline Energy range $(\mathrm{GeV})$ & 1 & 3 & 16 \\
Rep. rate $(\mathrm{Hz})$ & 15 & 15 & 15 \\
rf voltage per turn $(\mathrm{MV})$ & & 0.15 & 1.5 \\
Circumference $(\mathrm{m})$ & & 158 & 474 \\
Protons per bunch $\left(\times 10^{13}\right)$ & & 2.5 & 2.5 \\
Beam emittance [95\%] & & \\
$\quad(\pi$ mm mmrad) & 200 & 240 \\
Bunch area [95\%] (eV s) & & 1.5 & $<2.0$ \\
Incoherent tune shift @ Inj. & 0.39 & 0.39 \\
\hline \hline
\end{tabular}


second ring. With such large apertures in rapid-cycling synchrotrons, careful design of the beam pipes for both rings is required to manage eddy-current effects. Two approaches are under consideration. One is a thin Inconel pipe with water cooling and eddy-current coil corrections integrated on the pipe, as in the AGS booster. The other is a ceramic beam pipe with a conductor inside to carry beam-image currents, as in the Spallation Neutron Source situated at Rutherford Appleton Laboratory, UK.

The Fermilab linac presently delivers a $400 \mathrm{MeV}$ beam and is capable, with modest modifications, of accelerating as many as $3 \times 10^{13}$ protons per cycle at $15 \mathrm{~Hz}$ [101]. A significant upgrade is required in order to deliver $10^{14}$ protons at $1 \mathrm{GeV}$. The energy can be raised by appending additional side-coupled modules to the downstream end of the linac. Increasing the linac beam intensity probably means increasing both the beam current and the duration of the beam pulse. Injection into the first ring is by charge stripping of the $\mathrm{H}^{-}$beam; the incoming beam will be chopped and injected into preexisting buckets to achieve high capture efficiency.

The circumference of the second ring is set equal to that of the existing Fermilab booster. This choice provides several advantages. First, the new booster could occupy the same tunnel as a relocated booster; second, the beam-batch length from a full second ring matches that of the present booster, which simplifies matching to downstream machines for other programs. The output energy of $16 \mathrm{GeV}$ then results from an assumed dipole packing fraction of 0.575 and a peak dipole field of $1.3 \mathrm{~T}$, which is the highest dipole field that is consistent with straightforward, nonsaturating design of magnets having thin silicon-steel laminations. Driving such magnets into saturation would cause significant heating of the magnet yoke as well as potential problems with tracking between the dipoles and quadrupoles.

The prebooster also has $1.3 \mathrm{~T}$ dipole fields, and its circumference is one-third that of the new booster; it operates at an rf harmonic number $h=4$. The strategy for achieving the required short bunches at the target while alleviating space-charge effects in the rings is to start with four bunches occupying most of the circumference of the first small ring in order to keep the bunching factor large, and to do a bunch-shortening rotation in longitudinal phase space just before extraction from the second synchrotron. The four bunches are accelerated in the first ring to $3 \mathrm{GeV}$, then transferred bunch-to-bucket into the second ring with its harmonic number $h=12$. At that energy, the kinematic factor in the tune-shift formula (20) is large enough to compensate for the smaller bunching factor in the second ring. The transfer energy of $3 \mathrm{GeV}$ between the two rings roughly equalizes their space-charge tune shifts.

Both rings employ separated-function lattices with flexible momentum compaction in order to raise their transition energies above their respective extraction energies. This not only avoids having to accelerate a beam through transition, but also provides other advantages. Intense beams are not subject to certain instabilities such as the negative-mass instability below transition and empirically seem less susceptible to other instabilities such as the microwave instability. Also, the negative natural chromaticity is beneficial for stabilizing the beam below transition, thereby perhaps obviating the need for sextupole correctors, especially in the first ring. Having transition not too far above extraction also provides a substantial bucket area in which to accomplish beamshortening rf manipulations.

Several potential sources of instabilities in the rings have been examined [102]. Space charge is the main factor affecting the stability of the beams; the rings appear to be safe from longitudinal- and transverse-microwave instabilities. Of course, standard stabilizing methods such as active dampers are necessary to counteract some of the instabilities. Flexible momentum-compaction lattices would be useful not only to raise the transition energy above the extraction energy, but also to allow fast changes in the slip factor to facilitate bunch-narrowing manipulations at extraction time.

The magnet-power-supply circuit for each ring is a $15 \mathrm{~Hz}$ resonant system like that of the existing booster, with dipoles and quadrupoles electrically in series. This implies that the second ring will accelerate only one batch at a time from the first ring, which is all that the muon collider needs. Adding about $15 \%$ of second harmonic to the magnet ramp reduces the required peak accelerating voltage by about $25 \%$, which is probably worth doing, especially for the second ring with its large voltage requirement.

One of the advantages of a two-ring system is that the two rings divide the work of accelerating the beam. The rf system of the first ring is relatively modest because of its small circumference and small energy gain; that of the second ring is simplified because its high injection energy means a small rf-frequency swing [103].

ESME simulations of longitudinal motion show that the rms bunch length is $2 \mathrm{nsec}$ as desired after the bunch rotation that occurs just before extraction from the second synchrotron. The bunch rotation creates momentum spreads of about $2 \%$ with longitudinal emittances of about $2 \mathrm{eV}$ s per bunch. Such spreads would contribute a few $\mathrm{cm}$ in quadrature to the beam size for a short period before extraction. This is thought to be tolerable, given the large apertures that are required in any case. High injection energies help to alleviate these longitudinal effects, which result from space-charge voltages having the same $1 / \beta \gamma^{2}$ kinematic dependence as the transverse tune shifts.

\section{AGS upgrade}

The third study [95-98] is of an upgrade to the BNL AGS, which should produce bunches larger than those required for the muon collider, but at a lower repetition rate. The AGS presently produces $6 \times 10^{13}$ protons in eight 
bunches at $25 \mathrm{GeV}$ and $0.6 \mathrm{~Hz}$. A $2.5 \mathrm{GeV}$ accumulator ring in the AGS tunnel and AGS power-supply upgrade to $2.5 \mathrm{~Hz}$ operation would match the repetition rate to the 10 $\mathrm{Hz}$ repetition rate of the booster. This would generate 1 MW beam power. With an additional upgrade of the linac energy to $600 \mathrm{MeV}$, an intensity of $2 \times 10^{14}$ protons/pulse in four bunches of $5 \times 10^{13}$ at $25 \mathrm{GeV}$ and $2.5 \mathrm{~Hz}$ could be reached, raising the power to $2 \mathrm{MW}$. The upgrades to the AGS accelerator complex are summarized in Fig. 13. Other options are also under consideration, such as the addition of a second booster and $5 \mathrm{~Hz}$ operation, that would reach the baseline specification of $4 \mathrm{MW}$.

The AGS momentum acceptance of $\pm 3 \%$ requires that the longitudinal phase space occupied by one bunch be less than $4.5 \mathrm{eVs}$. This high bunch density in turn generates stringent demands on the earlier parts of the accelerator cycle. In particular, Landau damping from the beam momentum spread may guard against resistive wall instabilities during injection and longitudinal microwave instabilities after transition. Beam stability can be restored with a more powerful transverse-damping system and possibly a new low-impedance vacuum chamber. The transverse microwave instability is predicted to occur after transition crossing unless damped by Landau damping from incoherent tune spread or possibly high-frequency quadrupoles.

\section{Progress and open issues}

Conventional rf manipulations appear able to produce 1-2 ns proton bunches if enough rf voltage to overcome the space-charge forces is used, and the beam energy is far enough from transition so the final bunch rotation is fast. Both simulations and experimental work have been directed at demonstrating that a short pulse can be produced easily.

An experiment at the AGS has shown that bunches with $\sigma_{z}=2 \mathrm{~ns}$ can be produced near transition from bunches with $\sigma_{z} \sim 8 \mathrm{~ns}$ by bunch rotation [104,105]. In this experiment, the AGS was flattoped near transition $(\sim 7 \mathrm{GeV})$, while the $\gamma_{t}$-jump system was used to bring

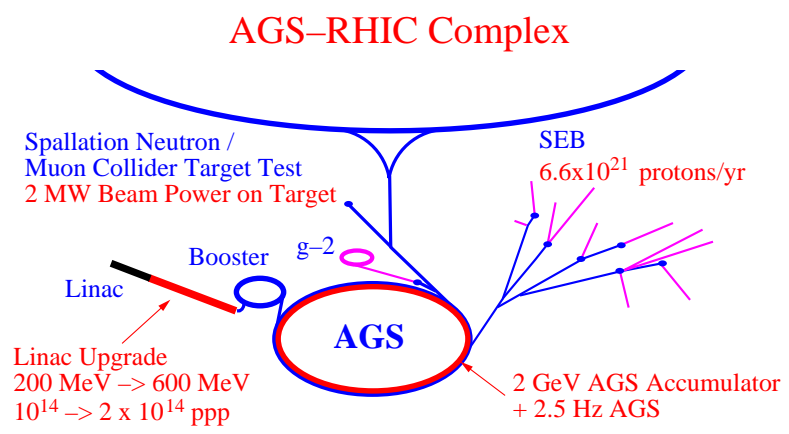

FIG. 13. (Color) The AGS-RHIC accelerator complex and a summary of possible intensity upgrades for the AGS. the transition energy suddenly to the beam energy, letting the bunch-energy spread expand and bunch length contract. The experiment also demonstrated that bunches are stable against microwave instabilities. In addition, the data were used to measure the lowest two orders of the momentum compaction factor.

The AGS bunch area, $1.5 \mathrm{eV} \mathrm{s}$, was comparable to that expected in the proton driver, but the bunch charge (though as large as $3-5 \times 10^{12}$ protons) was only about onetenth of that required by the muon target. The proton driver would use a flexible momentum compaction lattice which would allow tuning far from transition and permit a fast final bunch rotation [106]. In addition, the $\mathrm{rf}$ frequency would be higher than that of the AGS so the buckets (and bunches) would initially be only half as long. Thus bunch rotation could be expected to be easier with the new machine, which should compensate for the larger charge.

Simulations with the ESME code have also shown that $1-$ $2 \mathrm{~ns}$ bunches of $5 \times 10^{13}$ can be produced at extraction in a $16 \mathrm{GeV}$ ring with the rf and emittance shown in Table III.

The efficiency of capturing and accelerating a beam may be increased by compensation of the space-charge forces in the proton driver. The use of tunable inductive inserts in the ring vacuum chamber may permit active control and compensation of the longitudinal space charge below transition (since the inductive impedance is of the opposite sign to the capacitive space charge). Initial experiments at the KEK proton synchrotron and Los Alamos PSR [107] with short ferrite inserts appear to show a reduction in the synchrotron oscillation frequency shift caused by space charge and a decrease in the necessary rf voltage to maintain a given bunch intensity. Further experiments are needed to demonstrate this technique fully.

The high rf voltage and beam power and the relatively small size of the machine require high-gradient, high-power rf cavities. Fermilab, BNL, and KEK are collaborating on research and development of such types of cavities. This work includes the study of magnet alloys and hybrid cavities using both ferrite and new magnet alloys, high-power amplifiers, and beam-loading compensation.

The employment of barrier-bucket [108] rf cavities can effectively generate and manipulate a gap in the beam and reduce the space-charge effect. A successful test of this scheme has recently been completed [109], and two $40 \mathrm{kV}$ barrier cavities have been built by BNL and KEK and are being installed on the AGS. Another high-gradient barrier cavity using magnet alloys is under study at Fermilab.

\section{PION PRODUCTION, CAPTURE, AND PHASE ROTATION CHANNEL}

This section first discusses the choice of target technology and optimization of the target geometry and then 


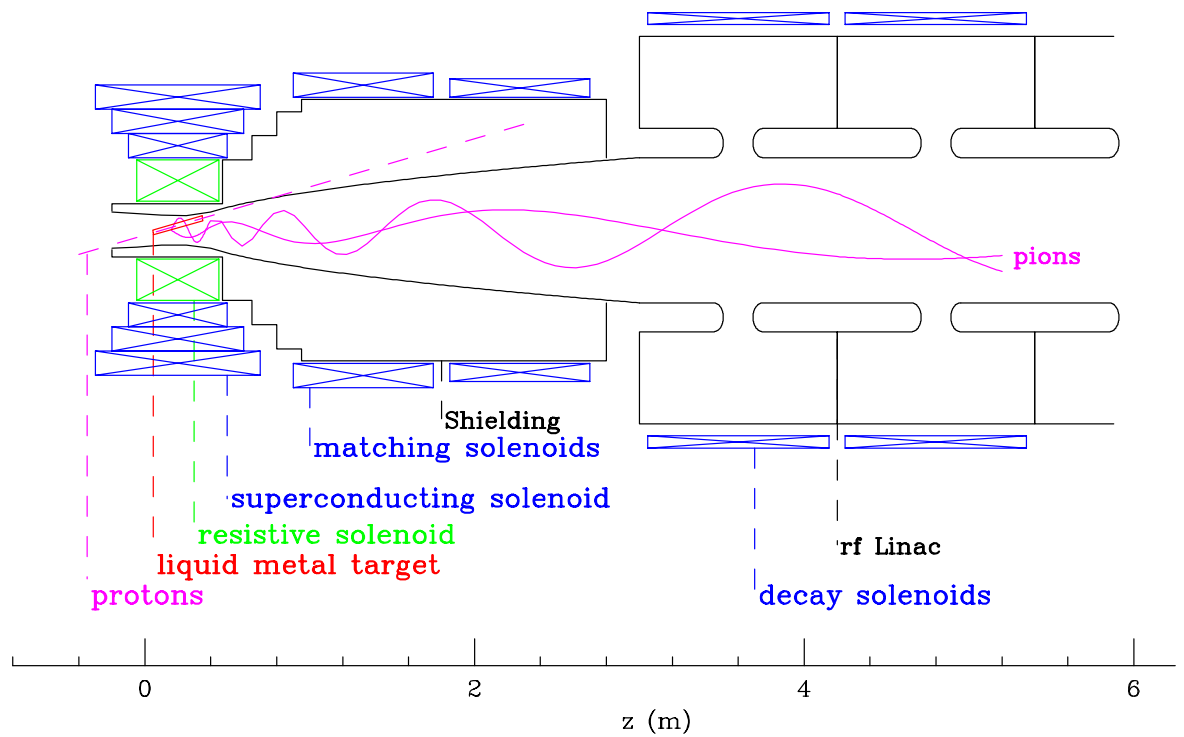

FIG. 14. (Color) Schematic view of pion production, capture, and initial phase rotation. A pulse of $16-30 \mathrm{GeV}$ protons is incident on a skewed target inside a high-field solenoid magnet followed by a decay and phase rotation channel.

describes design studies for the pion capture and phase rotation channel. Prospects for polarized muon beams are discussed in detail. The section concludes with an outline of an R\&D program for target and phase rotation issues.

Figure 14 gives an overview of the configuration for production of pions by a proton beam impinging on a long, transversely thin target, followed by capture of low-momentum, forward pions in a channel of solenoid magnets with rf cavities to compress the bunch energy while letting the bunch length grow. This arrangement performs the desired rotation of the beam.

\section{A. Pion production}

To achieve the luminosities for muon colliders presented in Table I, $2 \times 10^{12}$ (or $4 \times 10^{12}$ in the $100 \mathrm{GeV}$ COM case), muons of each sign must be delivered to the collider ring in each pulse. We estimate that a muon has a probability of only $1 / 4$ of surviving the processes of cooling and acceleration, due to losses in beam apertures or by decay. Thus, $0.8 \times 10^{13}$ muons $\left(1.6 \times 10^{13}\right.$ at $100 \mathrm{GeV}$ ) must exit the phase rotation channel each pulse. For pulses of $2.5 \times 10^{13}$ protons $\left(5 \times 10^{13}\right.$ for $\left.100 \mathrm{GeV}\right)$, this requires 0.3 muons per initial proton. Since the efficiency of the phase rotation channel is about $1 / 2$, this is equivalent to a capture of about 0.6 pions per proton: a very high efficiency.

The pions are produced in the interaction of the proton beam with the primary target. Extensive simulations have been performed for pion production from $8-30 \mathrm{GeV}$ proton beams on different target materials in a high-field solenoid [44,110-113]. Three different Monte Carlo codes [114-117] predict similar pion yields despite significant differences in their physics models. Some members of the Collaboration are involved in an AGS experiment BNL E-910 [118] to measure the yield of very low momentum pions, which will validate the codes in the critical kinematic region. This experiment ran for 14 weeks during the Spring of 1996 and has collected over $20 \times 10^{6}$ events, of which about a quarter are minimum bias triggers for inclusive cross section measurements.

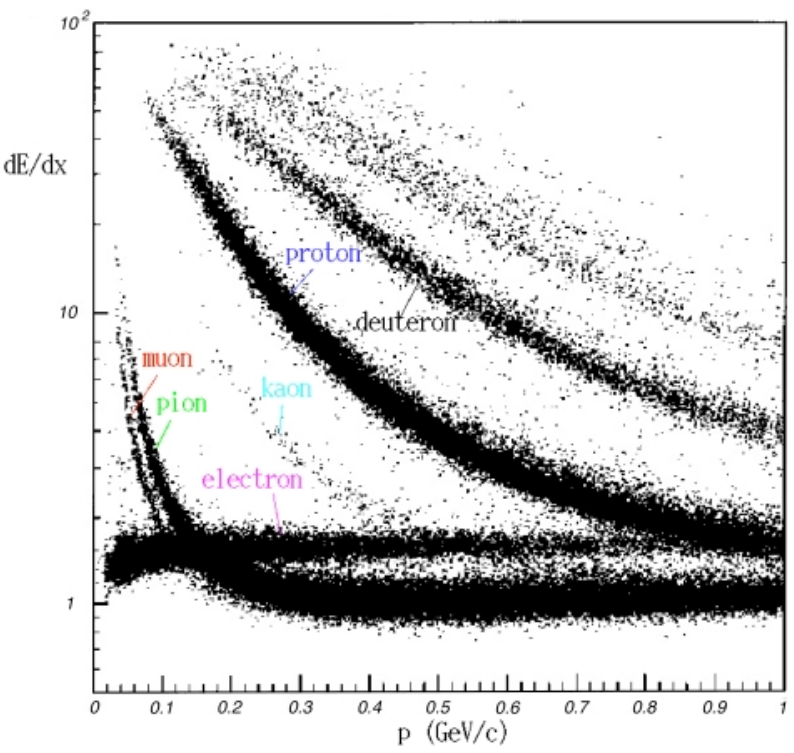

FIG. 15. (Color) $d E / d x$ curve in arbitrary units for low momentum tracks; the ionization energy loss is for tracks with 30 or more hits in the time projection chamber (TPC). The incident beam momentum is $18 \mathrm{GeV} / c$. From left to right the bands correspond to muons, pions, kaons, protons, and deuterium, respectively. Note the overlap of the (nearly horizontal) electron band with other species. 
The targets were varied in material $(\mathrm{Be}, \mathrm{Cu}, \mathrm{Au}$, and $\mathrm{U})$ and thickness $\left[2 \%-100 \%\right.$ interaction length $\left.\left(\lambda_{I}\right)\right]$, and three different beam momenta were used $(6,12.5$, and $18 \mathrm{GeV} / c$ ). Presently, the E910 Collaboration is doing a careful analysis of the large data sample obtained. Figure 15 shows the $d E / d x$ energy vs momentum for reconstructed tracks in the TPC; there is clear particle species separation [119].

The pion yield is greater for relatively high $Z$ materials, and, for these, the pion yield is maximal for longitudinal momenta of the same order as the average transverse momentum $(\approx 200 \mathrm{MeV} / c)$. Targets of varying composition $(6<Z<82)$, radii $(0.2-3 \mathrm{~cm})$, and thicknesses $(0.5-$ 3 nuclear interaction lengths) have been explored using a
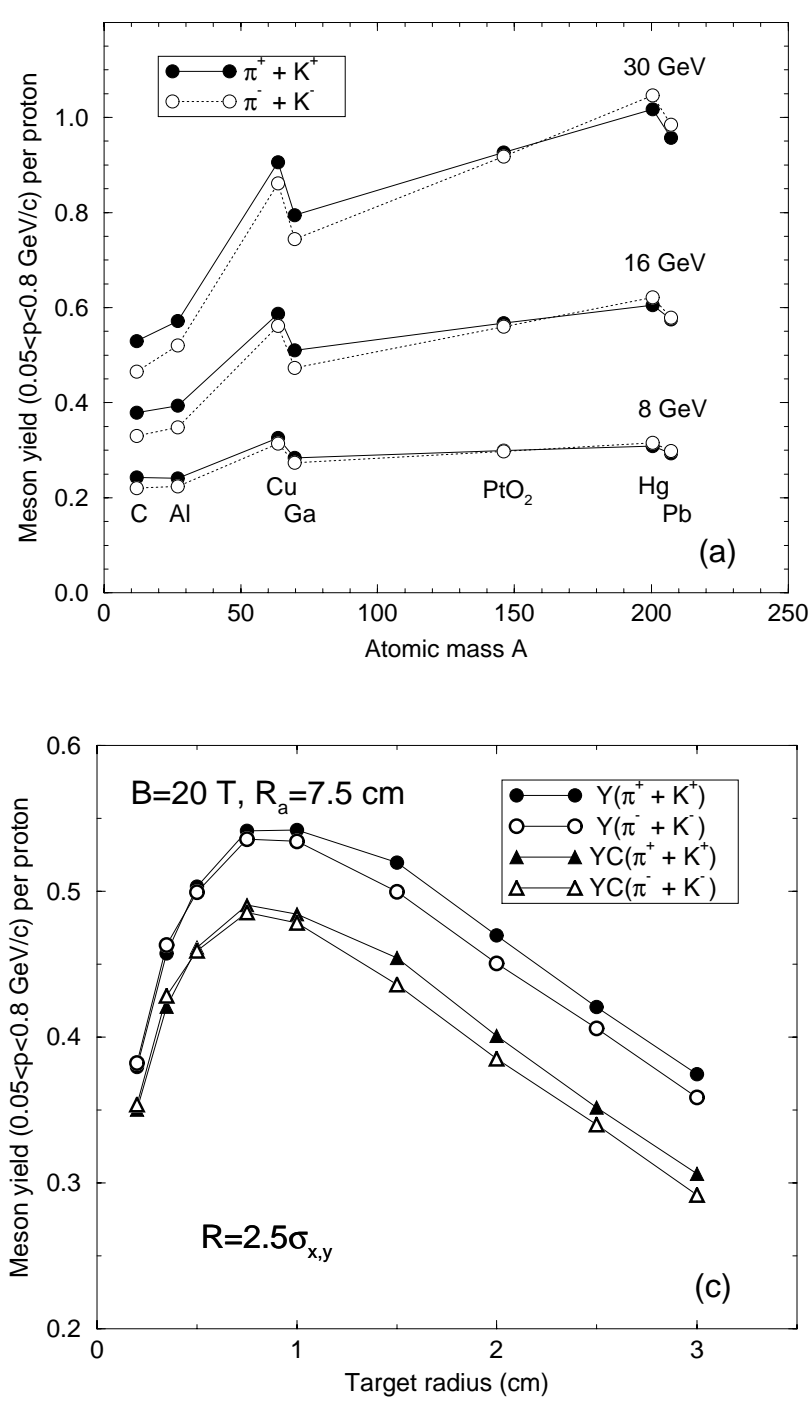

Monte Carlo simulation [111]. For a fixed number of interaction lengths, the pion yield per proton rises almost linearly with proton energy, and hence almost proportional to the energy deposited in the target. The yield is higher for medium- and high- $Z$ target materials, with a noticeable gain at $Z>26$ for $30 \mathrm{GeV}$ proton beams, but with only a minor effect for $E \leq 16 \mathrm{GeV}$. This is shown in Fig. 16 where results of detailed MARS13(98) [115] simulations are presented. The curves show the meson yield $(\pi+K)$ from the targets in the momentum interval $0.05 \leq P \leq 0.8 \mathrm{GeV} / c$ (labeled $\mathrm{Y}$ ) and the number of mesons that are both captured in the high-field solenoid and transported into the decay channel (labeled YC). The typical statistical error is a few percent.
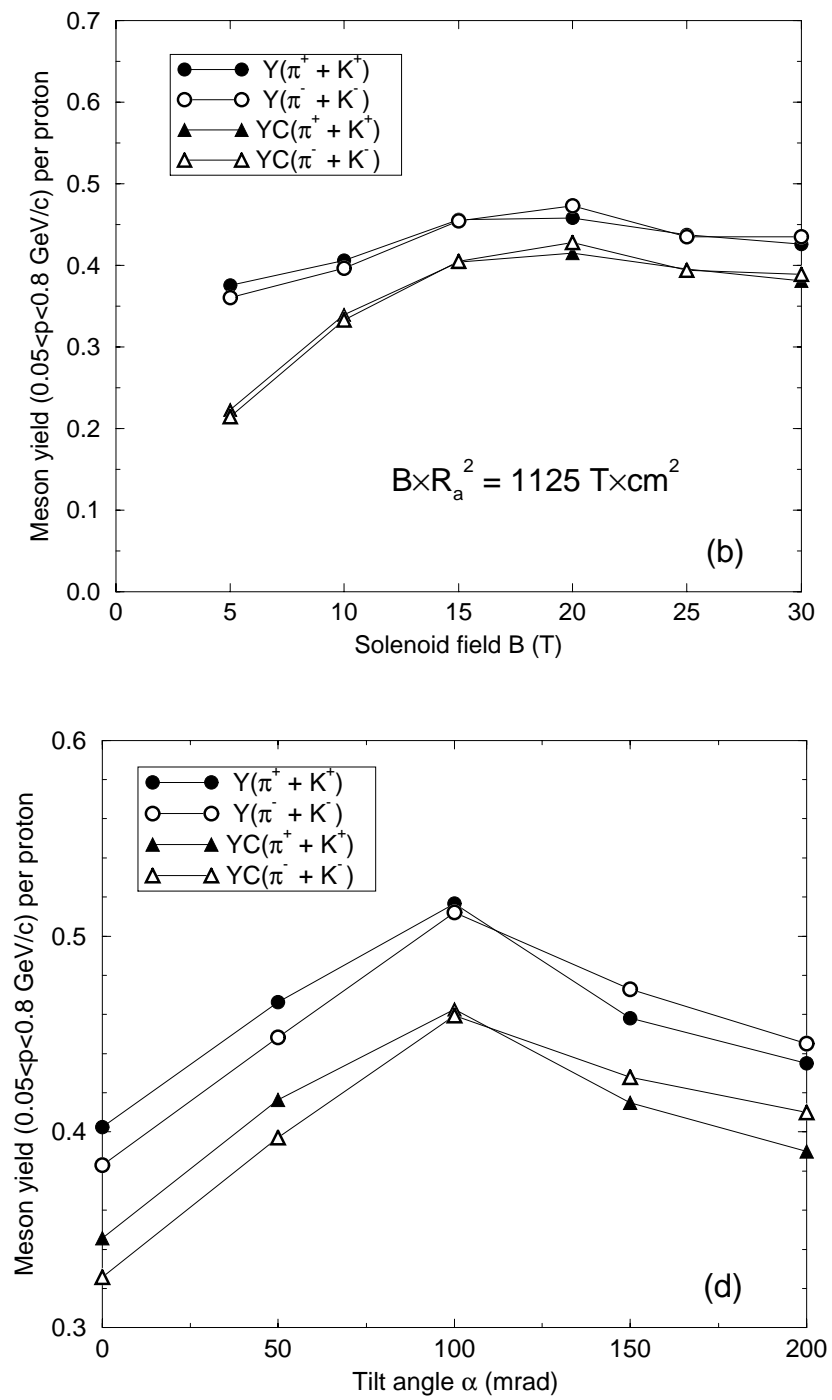

FIG. 16. Meson yield $(\pi+K)$ from different targets tilted by angle $\alpha$ in a solenoidal field $B$ of aperture $R_{a}$ as calculated with the MARS13(98) code. The target is aligned along the beam. The curves labeled YC show mesons that are transported into the decay channel. (a) Yield from a $1.5 \lambda_{I}, 1 \mathrm{~cm}$ radius target irradiated with 8,16 , and $30 \mathrm{GeV}$ proton beams $\left(\sigma_{x}=\sigma_{y}=4 \mathrm{~mm}\right)$ as a function of target atomic mass $\left(B=20 \mathrm{~T}, R_{a}=7.5 \mathrm{~cm}, \alpha=0\right)$. (b) Yield from a $3 \lambda_{I}, 1 \mathrm{~cm}$ radius gallium target tilted at $\alpha=150 \mathrm{mrad}$ in a $16 \mathrm{GeV}$ proton beam $\left(\sigma_{x}=\sigma_{y}=4 \mathrm{~mm}\right)$ vs solenoid field for a fixed adiabatic invariant $B R_{a}^{2}$. (c) Yield as a function of radius of a $3 \lambda_{I}$ gallium target in a $16 \mathrm{GeV}$ proton beam $\left(\sigma_{x}=\sigma_{y}=4 \mathrm{~mm}, B=20 \mathrm{~T}, R_{a}=7.5 \mathrm{~cm}, \alpha=100 \mathrm{mrad}\right)$. (d) Yield from a $3 \lambda_{I}, 1 \mathrm{~cm}$ radius gallium target vs tilt angle between the axis of the capture solenoid and the proton beam for a $16 \mathrm{GeV}$ proton beam $\left(\sigma_{x}=\sigma_{y}=4 \mathrm{~mm}, B=20 \mathrm{~T}, R_{a}=7.5 \mathrm{~cm}\right)$. 


\section{B. Target}

The target should be 2-3 interaction lengths long to maximize pion production. A high-density material is favored to minimize the size and cost of the capture solenoid magnet. Target radii larger than about $1 \mathrm{~cm}$ lead to lower pion rates due to reabsorption, while smaller diameter targets have less production from secondary interactions. Tilting the target by $100-150 \mathrm{mrad}$ minimizes loss of pions by absorption in the target after one turn on their helical trajectory $[50,120]$. Another advantage of the tilted target geometry is that the high energy and neutral components of the shower can be absorbed in a watercooled beam dump to the side of the focused beam.

About $30 \mathrm{~kJ}$ of energy is deposited in the target by each proton pulse (10\% of the beam energy). Hence, the target absorbs $400 \mathrm{~kW}$ of power at the $15 \mathrm{~Hz}$ pulse rate. Cooling of the target via contact with a thermal bath would lead to unacceptable absorption of pions, and radiative cooling is inadequate for such high power in a compact target. Therefore, the target must move so as to carry the energy deposited by the proton beam to a heat exchanger outside the solenoid channel.

Both moving solid metal and flowing liquid targets have been considered, with the latter as the currently preferred solution. A liquid is relatively easy to move, easy to cool, can be readily removed and replaced, and is the preferred target material for most spallation neutron sources under study. A liquid flowing in a pipe was considered, but experience at ISOLDE with short proton pulses [121] as well as simulations [122,123] suggest serious problems in shock damage to the pipe. An open liquid jet is thus proposed.

A jet of liquid mercury has been demonstrated [122] but not exposed to a beam. For our application, safety and other considerations favor the use of a low melting point lead alloy rather than mercury. Gallium alloys, though with lower density, are also being considered. Experimental and theoretical studies are underway to determine the consequences of beam shock heating of the liquid. It is expected that the jet will disperse after being exposed to the beam. The target station must survive damage resulting from the violence in this dispersion. This consideration will determine the minimum beam, and thus jet, radius.

For a conducting liquid jet in a strong magnetic field, as proposed, strong eddy currents will be induced in the jet, causing reaction forces that may disrupt its flow $[124,125]$. The forces induced are proportional to the square of the jet radius and set a maximum for this radius of order 5-10 mm. If this maximum is smaller than the minimum radius set by shock considerations, then multiple smaller beams and jets could be used; e.g., four jets of $5 \mathrm{~mm}$ radius with four beams with $2.5 \times 10^{13}$ protons per bunch. Other alternatives include targets made from insulating materials such as liquid $\mathrm{PtO}_{2}$ or $\mathrm{Re}_{2} \mathrm{O}_{3}$, slurries (e.g., Pt in water), or powders [126].
A moving solid metal target is not the current baseline solution, but is a serious possibility. In this case, the target could consist of a long flat band or hoop of coppernickel that moves along its length (as in a band saw) [127]. The band would be many meters in length, would be cooled by gas jets away from the target area, and would be supported and moved by rollers, as shown in Fig. 17.

The choice and parameters of the target are critical issues that need resolution. These can be resolved by experiments with a strong magnetic field and a beam, as discussed in Sec. IV H.

\section{Capture}

To capture all pions with transverse momenta $p_{T}$ less than their typical value of $200 \mathrm{MeV} / c$, the product of the capture solenoid field $B$ and its radius $R_{a}$ must be greater than $1.33 \mathrm{Tm}$. The use of a high field and small radius is preferred to minimize the corresponding transverse emittance, which is proportional to $B R^{2}$ : for a fixed transverse momentum capture this emittance is thus proportional to $R$. A field of $20 \mathrm{~T}$ and $7.5 \mathrm{~cm}$ radius was chosen on the basis of simulations described below. This gives $B R=1.5 \mathrm{~T} \mathrm{~m}, B R^{2}=0.1125 \mathrm{~T} \mathrm{~m}^{2}$, and a maximum transverse momentum capture of $p_{T}=$ $225 \mathrm{MeV} / c$.

A preliminary design [128] of the capture solenoid has an inner $6 \mathrm{~T}, 4 \mathrm{MW}$, water cooled, hollow conductor magnet with an inside diameter of $24 \mathrm{~cm}$ and an outside diameter of $60 \mathrm{~cm}$. There is space for a $4 \mathrm{~cm}$ thick, water cooled, heavy metal shield inside the coil. The outer superconducting magnet has three coils, with inside diameters of $60-80 \mathrm{~cm}$. It generates an additional $14 \mathrm{~T}$ of field at the target and provides the required tapered field to match into the decay channel. Such a hybrid solenoid has parameters compatible with those of existing magnets [129].

The $20 \mathrm{~T}$ capture solenoid is matched via a transfer solenoid [110] into a decay channel consisting of a system of superconducting solenoids with the same adiabatic

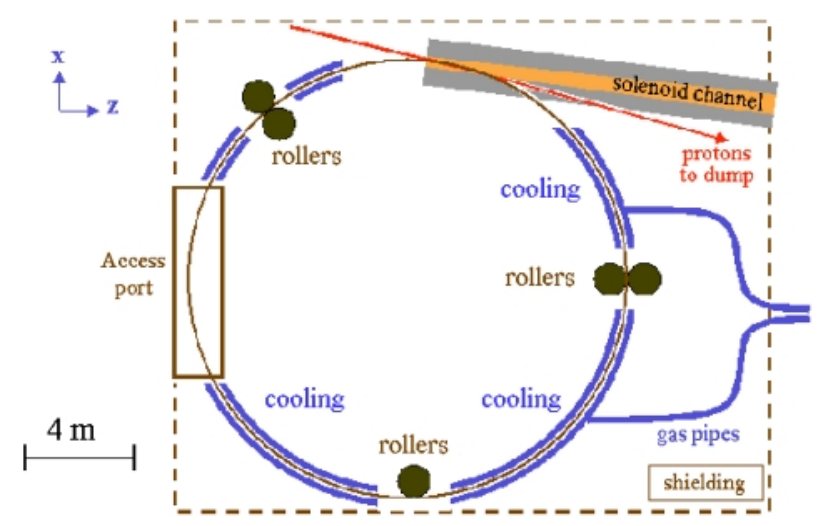

FIG. 17. (Color) Alternative concept of a solid metal target in the form of a rotating $\mathrm{Cu}-\mathrm{Ni}$ band. 
invariant $B R^{2} \propto R p_{T}$. Thus, for a $1.25 \mathrm{~T}$ decay channel, $B$ drops by a factor of 16 between the target and decay channel, $R$ and $p_{T}$ change by factors of 4 and $1 / 4$, respectively. This permits improved acceptance of transverse momentum within the decay channel, at the cost of an increased spread in longitudinal momentum. Figure 16(b) shows the meson yield as a function of field in the capture solenoid, with the radius of the capture solenoid adjusted to maintain the same $B R_{a}^{2}$ as in the decay channel. The optimum field is $20 \mathrm{~T}$ in the capture solenoid.

If the axis of the target is coincident with that of the solenoid field, then there is a relatively high probability that pions reenter the target after one cycle on their helical trajectory and are lost due to nuclear interactions. When the target and proton beam are set at an angle of $100-150 \mathrm{mrad}$ with respect to the field axis [111], the probability for such pion interactions at the target is reduced, and the overall production rate is increased by $60 \%$, as shown in Fig. 16(d).

In summary, the simulations indicate that a $20 \mathrm{~T}$ solenoid of $16 \mathrm{~cm}$ inside diameter surrounding a tilted target will capture about half of all produced pions. With target efficiency included, about 0.6 pions per proton will enter the pion decay channel [111].

\section{Phase rotation linac}

The pions, and the muons into which they decay, have a momentum distribution with an rms spread of approximately $100 \%$ and a peak at about $200 \mathrm{MeV} / c$. It would be difficult to handle such a wide spread in any subsequent system. A linac is thus introduced along the decay channel, with frequencies and phases chosen to decelerate the fast particles and accelerate the slow ones, i.e., to phase rotate the muon bunch. Several studies have been made of the design of this system, using differing ranges of rf frequency, delivering different final muon momenta, and differing final bunch lengths. In all cases, muon capture efficiencies of close to 0.3 muons per proton are obtained. Until the early stages of the ionization cooling have been designed, it is not yet possible to choose between them. Independent of the above choices is a question of the location of the focusing solenoid coils and rf cavity design, as discussed below in Sec. IV F.

\section{Lower energy, longer bunch example}

This example captures muons at a mean kinetic energy of $130 \mathrm{MeV}$. Table IV gives parameters of the linacs used. The gradients listed are relatively high for continuous low frequency systems, but far below the surface fields achieved in short pulses $(\approx 75 \mathrm{MV} / \mathrm{m}$ at $202 \mathrm{MHz}$ for $1 \mathrm{~ms}$ and an effective acceleration gradient of $10.7 \mathrm{MV} / \mathrm{m}$ on tank2 of the CERN lead injector [130]). GSI Laboratory at Darmstadt, Germany, is also testing 36
TABLE IV. Parameters of the lower energy phase rotation linacs.

\begin{tabular}{cccc}
\hline \hline Linac & $\begin{array}{c}\text { Length } \\
(\mathrm{m})\end{array}$ & $\begin{array}{c}\text { Frequency } \\
(\mathrm{MHz})\end{array}$ & $\begin{array}{c}\text { Gradient } \\
(\mathrm{MeV} / \mathrm{m})\end{array}$ \\
\hline 1 & 3 & 60 & 5 \\
2 & 29 & 30 & 4 \\
3 & 5 & 60 & 4 \\
4 & 5 & 37 & 4 \\
\hline \hline
\end{tabular}

$\mathrm{MHz}$ linac cavities for its injector and the expected peak gradient is $\approx 15 \mathrm{MV} / \mathrm{m}$ [131]. We expect that the greatest problem will be the development of sufficiently high power low frequency rf sources. Monte Carlo simulations [52], with the program MCM [132], were done using pion production calculated by ARC [114] for a copper target of $1 \mathrm{~cm}$ radius at an angle of $150 \mathrm{mrad}$. A uniform solenoidal field was assumed in the phase rotation, and the rf was approximated by a series of kicks.

Figure 18 shows the energy vs $c t$ at the end of the decay and phase rotation channel. The abscissa $c t$ is a measure of bunch length at the end of the channel: the total transit time of each $\pi / \mu$ is multiplied by the velocity of light and the total length of the channel is subtracted. Thus a fictitious reference particle at the center of the incident bunch at the target arrives at $c t=0 \mathrm{~m}$. A loose final bunch selection was defined with an energy $130 \pm 70 \mathrm{MeV}$ and bunch $c t$ from 3-11 m. With this selection, the rms energy spread is $16.5 \%$, the rms $c t$ is $1.7 \mathrm{~m}$, and there are 0.39 muons per incident proton. A tighter selection with an energy $130 \pm 35 \mathrm{MeV}$ and bunch $c t$ from $4-10 \mathrm{~m}$ gave an rms energy spread of $11.7 \%$, rms $c t$ of $1.3 \mathrm{~m}$, and contained 0.31 muons per incident proton.

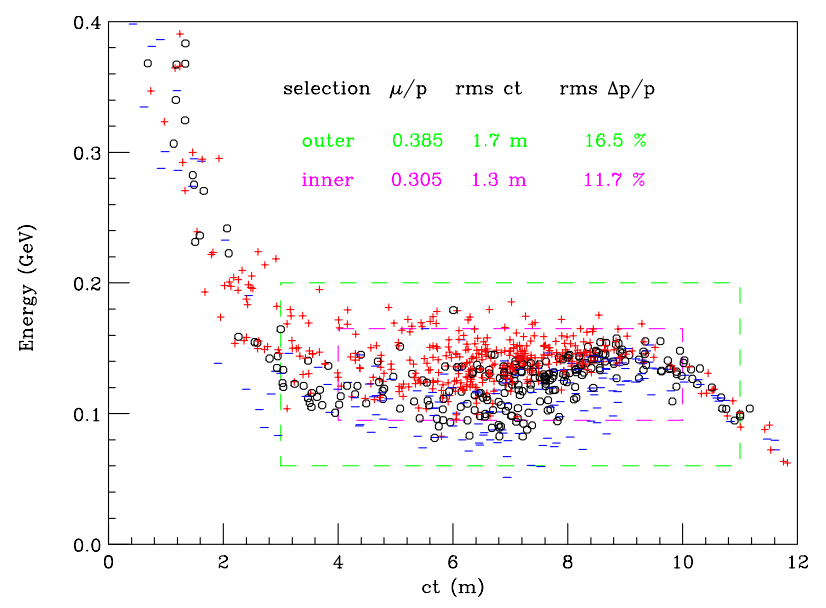

FIG. 18. (Color) Energy vs $c t$ of $\mu$ 's at the end of the lower energy phase rotation channel. The symbols,$+ \bigcirc$, and denote muons with polarization $P>\frac{1}{3},-\frac{1}{3}<P<\frac{1}{3}$, and $P<-\frac{1}{3}$, respectively. 


\section{Higher energy, shorter bunch example}

In this example the captured muons have a mean kinetic energy close to $320 \mathrm{MeV}$. It is based on a Monte Carlo study which uses the updated MARs pion production model [116] to generate pions created by $16 \mathrm{GeV}$ protons on a $36 \mathrm{~cm}$ long, $1 \mathrm{~cm}$ radius coaxial gallium target. Figure 19 shows the longitudinal phase space of the muons at the end of an $80 \mathrm{~m}$ long, $5 \mathrm{~T}$ solenoidal decay channel with cavities of frequency in the $30-90 \mathrm{MHz}$ range and acceleration gradients of $4-18 \mathrm{MeV} / \mathrm{m}$. A total of 0.33 muons per proton fall within the indicated cut $(6 \mathrm{~m} \times 300 \mathrm{MeV})$. The $\mathrm{rms}$ bunch length inside the cut is $148 \mathrm{~cm}$ and the rms energy spread is $62 \mathrm{MeV}$. The normalized $6 \mathrm{D}$ emittance is $217 \mathrm{~cm}^{3}$ and the transverse part is $1.86 \mathrm{~cm}$ (the normalized 6D emittance $\epsilon_{6, N}$ is defined in Sec. V).

A sample simulation with lithium hydride absorbers regularly spaced in the last $60 \mathrm{~m}$ of a $120 \mathrm{~m}$ decay channel and with compensating acceleration captures 0.3 muons with mean kinetic energy of about $380 \mathrm{MeV}$ in a $(6 \mathrm{~m} \times$ $300 \mathrm{MeV}$ ) window. The longitudinal phase space is about the same as in the previous example, but the transverse part shrinks to $0.95 \mathrm{~cm}$ due to ionization cooling which reduces the $6 \mathrm{D}$ phase space to $73.5 \mathrm{~cm}^{3}$.

\section{E. Use of both signs}

Protons on the target produce pions of both signs, and a solenoid will capture both, but the subsequent rf systems will have opposite effects on each sign. The proposed baseline approach uses two separate proton bunches to create separate positive and negative pion bunches and accepts the loss of half of the pions/muons during phase rotation.

If the pions can be charge separated with limited loss before the phase rotation cavities are reached, then higher luminosity may be obtained. The separation of charged pions in a curved solenoid decay line was studied in [110]. Because of the resulting dispersion in a bent solenoid, an initial beam of radius $R$ with maximum-tominimum momentum ratio $F$ will require a large beam pipe of radius $(1+F) R$ downstream to accommodate the separated beams. A septum can then be used to capture the two beams into separate channels. Typically, the reduction in yield for a curved solenoid compared to a straight solenoid is about $25 \%$ (due to the loss of very low and very high momentum pions to the walls or septum), but this must be weighed against the fact that both charge signs are captured for an overall net gain. A disadvantage is that this charge separation takes place over several meters of length during which time the beam spreads longitudinally. This makes capture in an rf phase rotation system difficult, although a large aperture cavity system could be incorporated in the bent solenoid region to alleviate this. The technique deserves further study and may be useful to consider as an intensity upgrade to a muon collection system.

\section{F. Solenoids and rf}

As noted above, capture using higher frequencies appears to be less efficient, and most studies now use

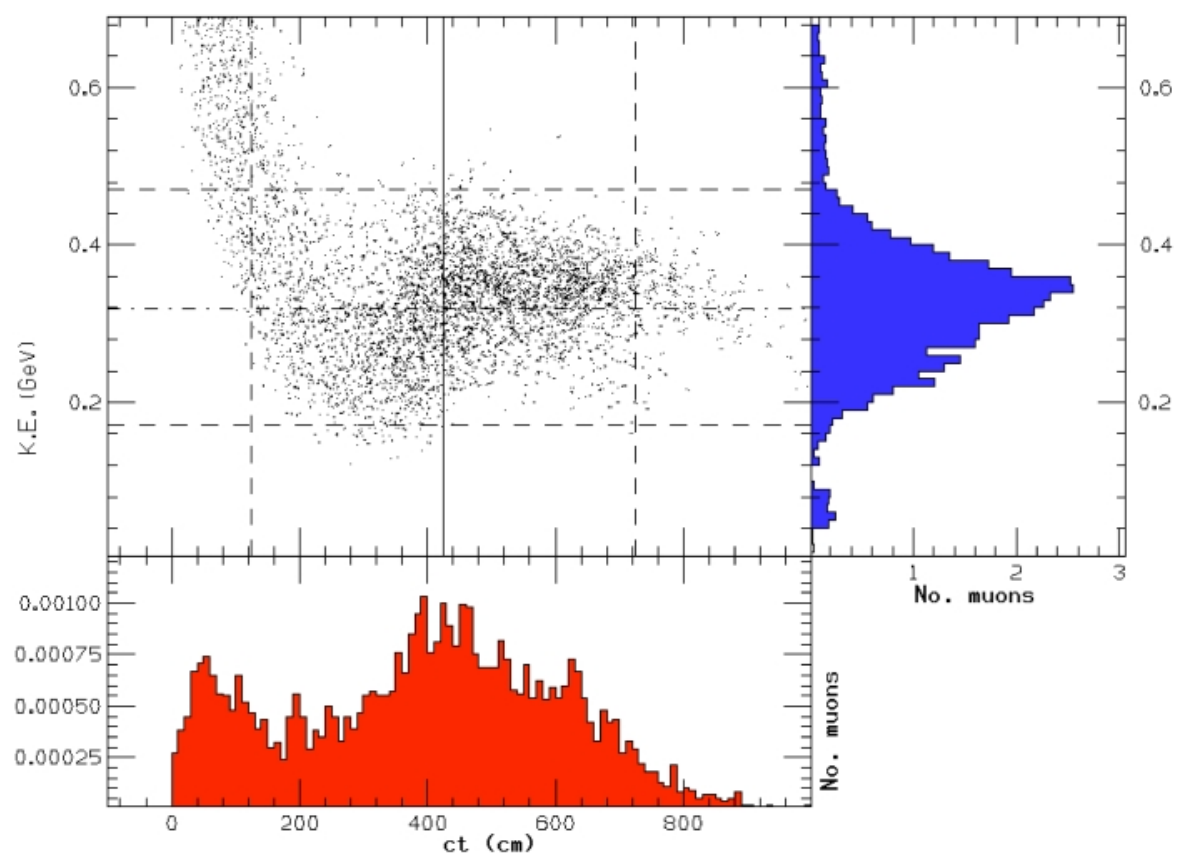

FIG. 19. (Color) Longitudinal phase space at the end of decay channel with projections onto time and energy axes per incident proton. The four dashed lines delineate the region deemed acceptable for the cooling channel. 
frequencies down to $30 \mathrm{MHz}$. Such cavities, when conventionally designed, are very large (about $6.6 \mathrm{~m}$ diameter). In the Snowmass study [133], a reentrant design reduced this diameter to $2.52 \mathrm{~m}$, but this is still large, and it was first assumed that the $5 \mathrm{~T}$ focusing solenoids would, for economic reasons, be placed within the irises of the cavities (see Fig. 20).

A study of transmission down a realistic system of iris located coils revealed betatron resonant excitation from the magnetic field periodicities, leading to significant particle loss. This was reduced by the use of more complicated coil shapes [133], smaller gaps, and shorter cavities, but remained a problem.

An alternative is to place continuous focusing coils outside the cavities as shown in Fig. 14. In this case, cost will be minimized with lower magnetic fields (1.25$2.5 \mathrm{~T}$ ) and correspondingly larger decay channel radii $(21-30 \mathrm{~cm})$. Studies are underway to determine the optimal solution.

\section{G. Polarization}

Polarization of the muon beams presents a significant physics advantage over the unpolarized case, since signal and background of electroweak processes usually come predominantly from different polarization states.

\section{Polarized muon production}

In the center-of-mass of a decaying pion, the outgoing muon is fully polarized $\left(P=-1\right.$ for $\mu^{+}$and +1 for $\left.\mu^{-}\right)$. In the lab system, the polarization depends on the decay angle $\theta_{d}$ and initial pion energy [134-136]. For pion kinetic energy larger than the pion mass, the average polarization is about $20 \%$, and, if nothing else is done, the polarization of the captured muons after the phase rotation system is approximately this value.

If higher polarization is required, some selection of muons from forward pion decays $\left(\cos \theta_{d} \rightarrow 1\right)$ is required. Figure 18 showed the polarization of the phase rotated muons. The polarization $\left(P>\frac{1}{3},-\frac{1}{3}<P<\frac{1}{3}\right.$, and $\left.P<-\frac{1}{3}\right)$ is marked by the symbols,$+ \bigcirc$, and - respectively. If a selection is made on the minimum energy of the muons, then greater polarization is obtained. The tighter the cut, the higher the polarization, but the less the fraction $F_{\text {surv }}$ of muons that survive. Figure 21 gives the results of a Monte Carlo study.

If this selection is made on both beams, and if the proton bunch intensity is maintained, then each muon bunch is reduced by the factor $F_{\text {surv }}$ and the luminosity would fall by $F_{\text {surv }}^{2}$. But if, instead, proton bunches are merged so as to obtain half as many bunches with twice the intensity, then the muon bunch intensity is maintained and the luminosity (and repetition rate) falls only as $F_{\text {surv }}$.

The luminosity could be maintained at the full unpolarized value if the proton source intensity could be increased. Such an increase in proton source intensity in the unpolarized case might be impractical because of the resultant excessive high energy muon beam power, but this restriction does not apply if the increase is used to offset losses in generating polarization.

Thus, the goal of high muon beam polarization may shift the parameters of the muon collider toward lower repetition rate and higher peak currents in the proton driver.

\section{Polarization preservation}

The preservation of muon polarization has been discussed in some detail in [137]. During the ionization cooling process the muons lose energy in material and have a spin-flip probability $\mathcal{P}$,

$$
\mathcal{P} \approx \int \frac{m_{e}}{m_{\mu}} \beta_{v}^{2} \frac{\delta E}{E},
$$

where $\beta_{v}$ is the normalized muon velocity and $\delta E / E$ is the fractional loss of energy due to ionization. In our case, the integrated energy loss is approximately $3 \mathrm{GeV}$ and the typical energy is $150 \mathrm{MeV}$, so the integrated spin-flip probability is close to $10 \%$. The change in polarization $\delta \mathcal{P} / \mathcal{P}$ is twice the spin-flip probability, so the reduction in polarization is approximately $20 \%$. This loss is included in Fig. 21.

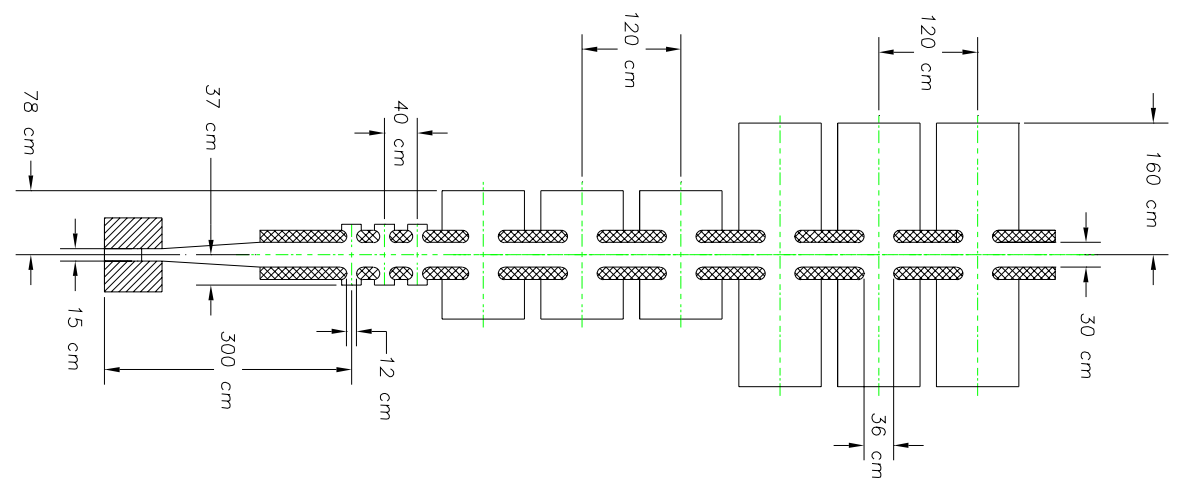

FIG. 20. (Color) Schematic of capture and phase rotation using rf cavities with superconducting solenoids (hatched areas) inside the irises. Three groups of three cavities operating at 90,50 , and $30 \mathrm{MHz}$ are shown from left to right, respectively. 


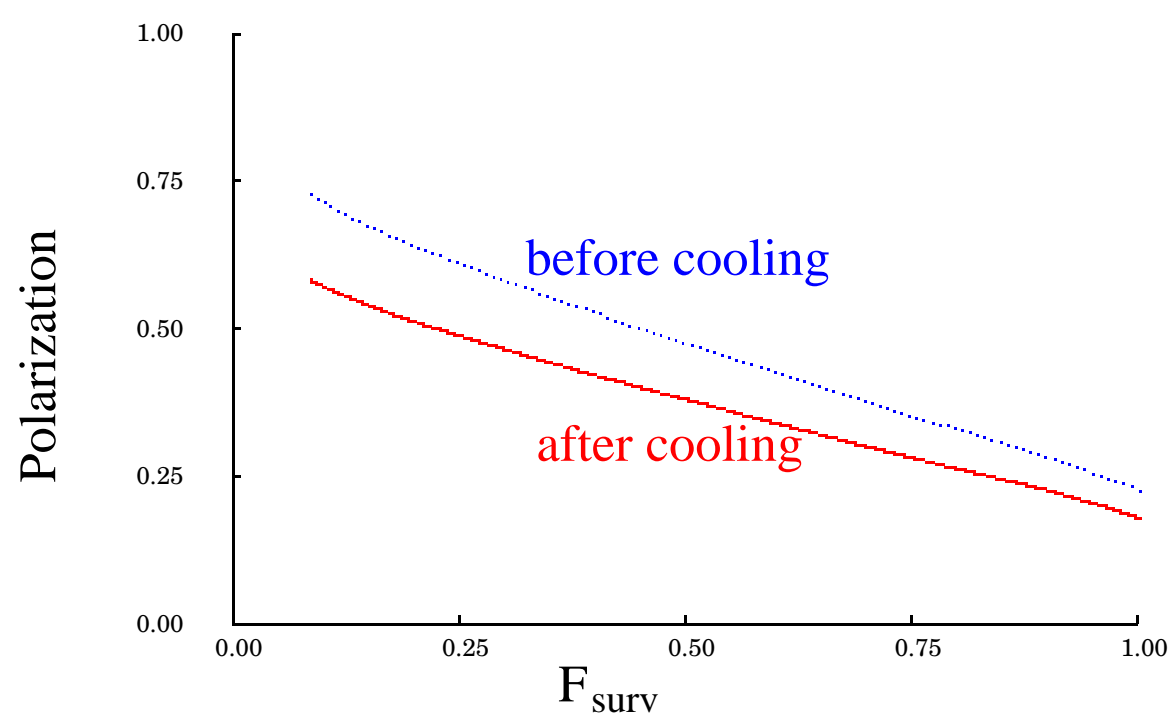

FIG. 21. (Color) Polarization vs fraction $F_{\text {surv }}$ of $\mu$ 's accepted.

During circulation in any ring, the muon spin, if initially longitudinal, will precess by $\gamma(g-2) / 2$ turns per revolution, where $(g-2) / 2$ is $1.166 \times 10^{-3}$. A given energy spread $\Delta \gamma / \gamma$ will introduce variations in these precessions and cause dilution of the polarization. But if the particles remain in the ring for an exact integer number of synchrotron oscillations, then their individual average $\gamma$ 's will be the same and no dilution will occur.

In the collider, bending can be performed with the spin orientation in the vertical direction, and the spin rotated into the longitudinal direction only for the interaction region. The design of such spin rotators appears relatively straightforward, but they are too long. This might be a preferred solution at high energies but is not practical in the $100 \mathrm{GeV}$ machine because of the constraint on the circumference of the ring imposed by the muon lifetime. An alternative is to use such a small energy spread, as in the Higgs factory, that although the polarization vector precesses, the beam polarization does not become significantly diluted. In addition, calibration of the Higgs factory collider energy to one part in $10^{6}$ [12] requires the spins to precess continuously from turn to turn.

\section{H. R\&D program}

An $R \& D$ program is underway to continue theoretical studies (optimization of pion production and capture) and to clarify several critical issues related to targetry and phase rotation [138]. A jet of the room temperature eutectic liquid alloy of Ga-Sn will be exposed to nanosecond pulses of $1.5 \times 10^{13} 24 \mathrm{GeV}$ protons at the Brookhaven AGS to study the effect of the resulting pressure wave on the liquid. The same jet will also be used in conjunction with a $20 \mathrm{~T}, 20 \mathrm{~cm}$ bore resistive magnet at the National High Magnetic Field Laboratory (Tallahassee, FL) to study the effect of eddy currents on jet propagation. Then, a pulsed, $20 \mathrm{~T}$ magnet will be added to the BNL test station to explore the full configuration of jet, magnet, and pulsed proton beam. Also, a $70 \mathrm{MHz}$ rf cavity will be exposed to the intense flux of secondary particles downstream of the target and $20 \mathrm{~T}$ magnet to determine viable operating parameters for the first phase rotation cavity. The complete configuration of the targetry experiment is sketched in Fig. 22.

The first two studies should be accomplished during 1999, and the third and fourth in the years 2000 and 2001.

\section{IONIZATION COOLING}

\section{A. Introduction}

The design of an efficient and practical cooling system is one of the major challenges for the muon collider project.

For a high luminosity collider, the $6 \mathrm{D}$ phase space volume occupied by the muon beam must be reduced by a factor of $10^{5}-10^{6}$. Furthermore, this phase space reduction must be done within a time that is not long compared to the muon lifetime ( $\mu$ lifetime $\approx 2 \mu \mathrm{s}$ ). Cooling by synchrotron radiation, conventional stochastic cooling, and conventional electron cooling are all too slow. Optical stochastic cooling [139], electron cooling in a plasma discharge [140], and cooling in a crystal lattice $[141,142]$ are being studied, but appear technologically difficult. The new method proposed for cooling muons is ionization cooling. This technique $[16,18,20,143]$ is uniquely applicable to muons because of their minimal interaction with matter. It is a method that seems relatively straightforward in principle, but has proven quite challenging to implement in practice 




FIG. 22. (Color) Plan view of the full configuration of the targetry experiment.

Ionization cooling involves passing the beam through some material in which the muons lose both transverse and longitudinal momentum by ionization energy loss, commonly referred to as $d E / d x$. The longitudinal muon momentum is then restored by reacceleration, leaving a net loss of transverse momentum (transverse cooling). The process is repeated many times to achieve a large cooling factor.

The energy spread can be reduced by introducing a transverse variation in the absorber density or thickness (e.g., a wedge) at a location where there is dispersion (the transverse position is energy dependent). This method results in a corresponding increase of transverse phase space and is thus an exchange of longitudinal and transverse emittances. With transverse cooling, this allows cooling in all dimensions.

We define the rms normalized emittance as

$$
\epsilon_{i, N}=\sqrt{\left\langle\delta r_{i}^{2}\right\rangle\left\langle\delta p_{i}^{2}\right\rangle-\left\langle\delta r_{i} \delta p_{i}\right\rangle^{2}} / m_{\mu} c,
$$

where $r_{i}$ and $p_{i}$ are the beam canonical conjugate variables with $i=1,2,3$ denoting the $x, y$, and $z$ directions, and $\langle\cdots\rangle$ indicates statistical averaging over the particles. The operator $\delta$ denotes the deviation from the average, so that $\delta r_{i}=r_{i}-\left\langle r_{i}\right\rangle$ and likewise for $\delta p_{i}$. The appropriate figure of merit for cooling is the final value of the $6 \mathrm{D}$ relativistically invariant emittance $\epsilon_{6, N}$, which is proportional to the area in the $6 \mathrm{D}$ phase space $\left(x, y, z, p_{x}, p_{y}, p_{z}\right)$ since, to a fairly good approximation, it is preserved during acceleration and storage in the collider ring. This quantity is the square root of the determinant of a general quadratic moment matrix containing all possible correlations. However, until the nature and practical implications of these correlations are understood, it is more conservative to ignore the correlations and use the following simplified expression for 6D normalized emittance:

$$
\epsilon_{6, N} \approx \epsilon_{x, N} \times \epsilon_{y, N} \times \epsilon_{z, N} .
$$

Theoretical studies have shown that, assuming realistic parameters for the cooling hardware, ionization cooling can be expected to reduce the phase space volume occupied by the initial muon beam by a factor of $10^{5}-10^{6}$. A complete cooling channel would consist of 20-30 cooling stages, each stage yielding about a factor of 2 in $6 \mathrm{D}$ phase space reduction.

It is recognized that understanding the feasibility of constructing a muon ionization cooling channel is on the critical path to determining the viability of the whole muon collider concept. The muon cooling channel is the most novel part of a muon collider complex. Steady progress has been made both in improving the design of sections of the channel and in adding detail to the computer simulations. A vigorous experimental program is needed to verify and benchmark the computer simulations.

The following parts of this section briefly describe the physics underling the process of ionization cooling. We will show results of simulations for some chosen examples and outline a six year R\&D program to demonstrate the feasibility of using ionization cooling techniques.

\section{B. Cooling theory}

In ionization cooling, the beam loses both transverse and longitudinal momentum as it passes through a material. At the same time its emittance is increased due to stochastic multiple scattering and Landau straggling. The longitudinal momentum can be restored by reacceleration, leaving a net loss of transverse momentum.

The approximate equation for transverse cooling in a step $d s$ along the particle's orbit is $[13,18,20,24,144,145]$

$$
\frac{d \epsilon_{N}}{d s}=-\frac{1}{\beta^{2}} \frac{d E_{\mu}}{d s} \frac{\epsilon_{N}}{E_{\mu}}+\frac{\beta_{\perp}(0.014 \mathrm{GeV})^{2}}{2 \beta^{3} E_{\mu} m_{\mu} L_{R}},
$$

where $\beta$ is the normalized velocity, $E_{\mu}$ is the total energy, $m_{\mu}$ is the muon mass, $\epsilon_{N}$ is the normalized transverse emittance, $\beta_{\perp}$ is the betatron function at the absorber, $d E_{\mu} / d s$ is the energy loss per unit length, and $L_{R}$ is the radiation length of the material. The betatron function 
is determined by the strengths of the elements in the focusing lattice [146]. Together with the beam emittance this function determines the local size and divergence of the beam. Note that the energy loss $d E_{\mu} / d s$ is defined here as a positive quantity, unlike the convention often used in particle physics. The first term in this equation is the cooling term, and the second describes the heating due to multiple scattering. The heating term is minimized if $\beta_{\perp}$ is small (strong focusing) and $L_{R}$ is large (a low- $Z$ absorber).

The minimum, normalized transverse emittance that can be achieved for a given absorber in a given focusing field is reached when the cooling rate equals the heating rate in Eq. (24),

$$
\epsilon_{N, \min }=\frac{\beta_{\perp}(14 \mathrm{MeV})^{2}}{2 \beta m_{\mu} \frac{d E_{\mu}}{d s} L_{R}} .
$$

For a relativistic muon in liquid hydrogen with a betatron focusing value of $8 \mathrm{~cm}$, which corresponds roughly to confinement in a $15 \mathrm{~T}$ solenoidal field, the minimum achievable emittance is about $340 \mathrm{~mm}$ mrad.

The equation for energy spread is $[18,24,25]$

$$
\frac{d\left(\Delta E_{\mu}\right)^{2}}{d s}=-2 \frac{d\left(\frac{d E_{\mu}}{d s}\right)}{d E_{\mu}}\left\langle\left(\Delta E_{\mu}\right)^{2}\right\rangle+\frac{d\left(\Delta E_{\mu}\right)_{\text {stragg }}^{2}}{d s}
$$

where the first term describes the cooling (or heating) due to energy loss, and the second term describes the heating due to straggling. $\Delta E_{\mu}$ is the rms spread in the energy of the beam.

Ionization cooling of muons seems relatively straightforward in theory, but will require extensive simulation studies and hardware development for its optimization. There are practical problems in designing lattices that can transport and focus the large emittance beam. There will also be effects from space charge and wake fields.

We have developed a number of tools for studying the ionization cooling process. First, the basic theory was used to identify the most promising beam properties, material type, and focusing arrangements for cooling. Given the practical limits on magnetic field strengths, this gives an estimate of the minimum achievable emittance for a given configuration. Next, the differential equations for cooling and heating described above were incorporated into a computer code. Allowance for the shifts in the betatron phase advance due to space charge and aberrations was included. This code was used to develop an overall cooling scenario, which broke the cooling system into a number of stages, and determined the properties of the beam, rf cavities, and focusing lattice at each stage.

Finally, several tracking codes were either written or modified to study the cooling process in detail. Two new codes (SIMUCOOL [147] and ICOOL [148,149]) use Monte Carlo techniques to track particles one at a time through the cooling system. All the codes attempt to include all relevant physical processes to some degree (e.g., energy loss, straggling, multiple scattering) and use Maxwellian models of the focusing fields. They do not yet take into account any space charge or wake field effects. In addition, we have also used a modified version of PARMELA [150] for tracking, which does include space charge effects, and a double precision version of GEANT [151,152].

We have recently developed [153] a model of beam cooling based on a second-order moment expansion. A computer code solving the equations for transverse cooling gives results that agree with tracking codes. The code is being extended to include energy spread and bends. It is very fast and is appropriate for preliminary design and optimization of the cooling channel. All of these codes are actively being updated and optimized for studying the cooling problem.

\section{Cooling system}

The cooling is obtained in a series of cooling stages. Each stage consists of a succession of the following components: (i) transverse cooling sections using materials in a strong focusing (low $\beta_{\perp}$ ) environment alternated with linear accelerators, (ii) emittance exchange in lattices that generate dispersion, with absorbing wedges to reduce momentum spread, and (iii) matching sections to optimize the transmission and cooling parameters of the following section.

The question of the best energy to use for cooling has been discussed in detail in Ref. [154]. For the set of parameters of interest, a kinetic energy close to $100 \mathrm{MeV}$ appears optimal. At higher energies, weaker focusing raises the heating term from Coulomb scattering and more acceleration is required for a given amount of cooling. At lower energies, the beam divergence becomes large and the rise of $d E / d x$ with falling energy causes a greater increase in energy spread. There can be an advantage, initially, of using a somewhat higher energy to reduce the beam dimensions and bucket length; at the end, the energy can be dropped to attain the lowest transverse emittances at the expense of longitudinal emittance. In the examples that follow it is seen that the simulated transverse cooling stages lower the 6D emittance by a factor of about 2 . Since the required total $6 \mathrm{D}$ cooling is $O\left(10^{6}\right)$, about 20 such stages are required.

We have performed calculations of complete cooling systems for the Higgs factory and for a high energy collider. These calculations are based on theoretical models of the expected cooling performance. They give an indication of the system dimensions, magnet strengths, rf frequencies and gradients, and beam parameters that will be required in a cooling system. The calculations suggest that the required cooling for a Higgs factory could be achieved in 25 stages, while the high energy collider would require 28 stages. Emittances and energies as a 
function of stage are shown in Fig. 23. The sequence can be considered to consist of three parts:

(i) For the first 12 stages the primary effort is to cool in the longitudinal direction in order to reduce the bunch lengths and allow higher frequency rf to be employed. Some transverse cooling is needed in order to reduce the transverse dimensions of the beam and allow it to fit through the smaller irises at the higher frequencies. In this example, for the first stage, an energy of $300 \mathrm{MeV}$ was used to calculate the reduction on the momentum spread and transverse beam dimensions. It is recognized that designing an emittance exchange at the beginning of the system that can simultaneously accept the large initial phase space of the beam and reduce the longitudinal emittance will be a major challenge. In later stages the energy is closer to $100 \mathrm{MeV}$. Solenoid focusing was assumed in all stages, with an initial field of the order of $1 \mathrm{~T}$ rising to about $3 \mathrm{~T}$ at the end.

(ii) In the second part (in this example, stages 1325) the $6 \mathrm{D}$ emittance is reduced as far as possible. For the case of a low momentum spread Higgs collider, the required beam parameters are now achieved and the third part is not required. In this example, $80 \mathrm{MeV}$ energy was used for all stages. Solenoid focusing was used in all but the last two stages, where lithium lenses were assumed.

(iii) For the higher luminosity and higher energy colliders, further reduction in transverse emittance is required, but this can be obtained without reduction of the $6 \mathrm{D}$ phase space, by allowing the longitudinal phase

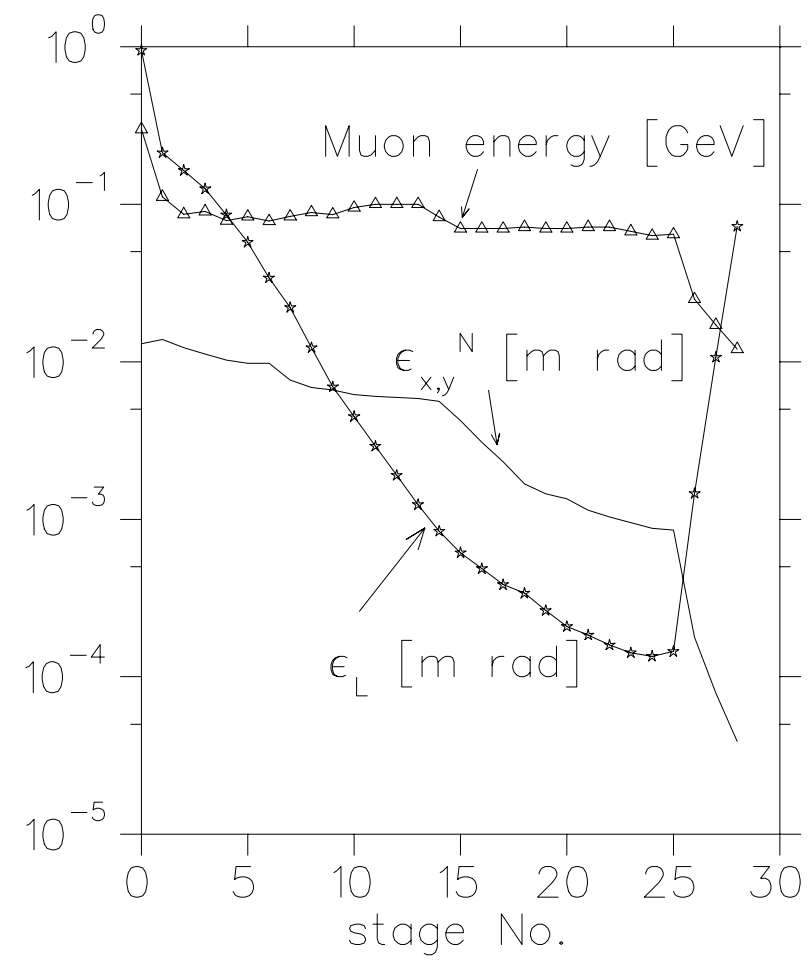

FIG. 23. Transverse $\left(\epsilon_{x, y}\right)$, longitudinal emittance $\left(\epsilon_{L}\right)$, and beam energy vs stage number in the cooling sequence. space to grow. This exchange of emittances is, in this example, achieved by reducing the energy to near $10 \mathrm{MeV}$ in two long lithium lens cooling stages. The same effect could probably be achieved at similar energy, by using a hydrogen absorber with solenoid focusing. It might also be possible by using wedges.

The total length of the system would be of the order of $600 \mathrm{~m}$, and the total acceleration required would be approximately $6 \mathrm{GeV}$. The fraction of muons remaining at the end of the cooling system is estimated to be $\approx 60 \%$. It must be emphasized that this sequence was derived without simulation of the individual stages. It serves, however, to guide the choice of stages to study in detail.

Three transverse cooling examples have been designed and simulated. The first uses $1.25 \mathrm{~T}$ solenoids to cool the very large emittance beam coming from the phase rotation channel. The muon beam at the end of the decay channel is very intense, with approximately $7.5 \times$ $10^{12}$ muons/bunch, but with a large normalized transverse emittance $\left[\epsilon_{x, N}(\mathrm{rms}) \approx 15 \times 10^{3} \pi \mathrm{mm} \mathrm{mrad}\right]$ and a large normalized longitudinal emittance $\left[\epsilon_{z, N}(\mathrm{rms}) \approx\right.$ $612 \pi \mathrm{mm}]$. The second example would lie toward the end of a full cooling sequence and uses $15 \mathrm{~T}$ solenoids. The third example, using $31 \mathrm{~T}$ solenoids, meets the requirements for the Higgs factory and could be the final cooling stage for this machine.

The baseline solution for emittance exchange involves the use of bent solenoids to generate dispersion and wedges of hydrogen or $\mathrm{LiH}$ to reduce the energy spread. A simulated example is given for exchange that would be needed after the $15 \mathrm{~T}$ transverse cooling case.

A lithium lens solution may prove more economical for the final stages, and might allow even lower emittances to be obtained. In this case, the lithium lens serves simultaneously to maintain the low $\beta_{\perp}$, and provide $d E / d x$ for cooling. Similar lenses, with surface fields of $10 \mathrm{~T}$, were developed at Novosibirsk (BINP) and have been used, at low repetition rates, as focusing elements at FNAL and CERN [7,155-158]. Lenses for the cooling application, which would operate at $15 \mathrm{~Hz}$, would need to employ flowing liquid lithium to provide adequate thermal cooling. Higher surface fields would also be desirable.

Studies have simulated cooling in multiple lithium lenses and have shown cooling through several orders of magnitude [11]. But these studies have, so far, used ideal matching and acceleration. Cooling is also being studied in beam recirculators, which could lead to reduction of costs of the cooling section $[159,160]$, but full simulations with all higher order effects have not yet been successfully demonstrated.

\section{15 T solenoid transverse cooling example}

The lattice consists of 11 identical $2 \mathrm{~m}$ long cells. In each cell there is a liquid hydrogen absorber $(64 \mathrm{~cm}$ long, $10 \mathrm{~cm}$ diameter) in the $15 \mathrm{~T}$ solenoid focusing magnet 
(64 cm long, $12 \mathrm{~cm}$ diameter). The direction of the fields in the magnets alternates from one cell to the next. Between the $15 \mathrm{~T}$ solenoids there are magnetic matching sections (1.3 m long, $32 \mathrm{~cm}$ inside diameter) where the field is lowered and then reversed. Inside the matching sections are short, $805 \mathrm{MHz}$, high gradient $(36 \mathrm{MeV} / \mathrm{m})$ linacs. Figure 24 shows the cross section of one cell of such a system, together with the betatron function, and the magnetic field along the axis. For convenience in modeling, the section shown in Fig. 24(a) starts and ends symmetrically in the middle of hydrogen absorber regions at the location of the peaks in the axial magnetic field. In practice each cell would start at the beginning of the hydrogen region and extend to the end of the rf module.

A GEANT simulation of muons traversing a section of the cooling channel is shown in Fig. 25.

Additional simulations were performed [161,162] using the program ICOOL. The only likely significant effects which are not yet included are space charge and wake fields. Analytic calculations for particle bunches in free space indicate that these effects should, for the later stages, be significant but not overwhelming.

A full simulation must be done before we are assured that no problems exist. Particles are introduced with transverse and longitudinal emittance $(186 \mathrm{MeV} / c$, $1400 \pi \mathrm{mm}$ mrad transverse, and $1.1 \pi \mathrm{mm}$ longitudinal), together with a number of naturally occurring correlations. First, the particles are given the angular momentum appropriate for the starting axial magnetic field. Second, particles with large initial radius $r_{0}$ and/or divergence $\theta_{0}$ have longer path lengths in a solenoidal field and tend to spread out with time. This can be parameterized by defining an initial transverse amplitude

$$
A^{2}=\frac{r_{0}^{2}}{\beta_{\perp}^{2}}+\theta_{0}^{2} .
$$

The temporal spreading can be minimized by introducing an initial correlation between $p_{z}$ and $A^{2}$ that equalizes the forward velocity of the initial particles. This correlation causes the average momentum of the beam to grow from the reference value of $186 \mathrm{MeV} / c$ to $\approx 195 \mathrm{MeV} / c$. Last, a distortion of the longitudinal bunch distribution can be introduced to reflect the asymmetric nature of the "alpha"-shaped rf bucket.

Figure 26(a) shows the average momentum of the beam as a function of distance along the channel. The momentum drops as the beam crosses the liquid hydrogen absorbers. The gradient and phase of the rf cavities have been adjusted so that the reacceleration given to the reference particle equals the mean energy loss. This causes the average momentum of the beam to remain in a narrow band around $195 \mathrm{MeV} / c$. Figure 26(b) shows the mechanical and canonical angular momenta as a function of distance along the channel. The mechanical angular momentum shows the rotational motion of the beam around the axial solenoidal field. It periodically reverses sign when the solenoids alternate direction. The canonical angular momentum is defined such that it removes the axial field dependence [162]. Without the absorbers, the beam would have a constant (0) value for the canonical
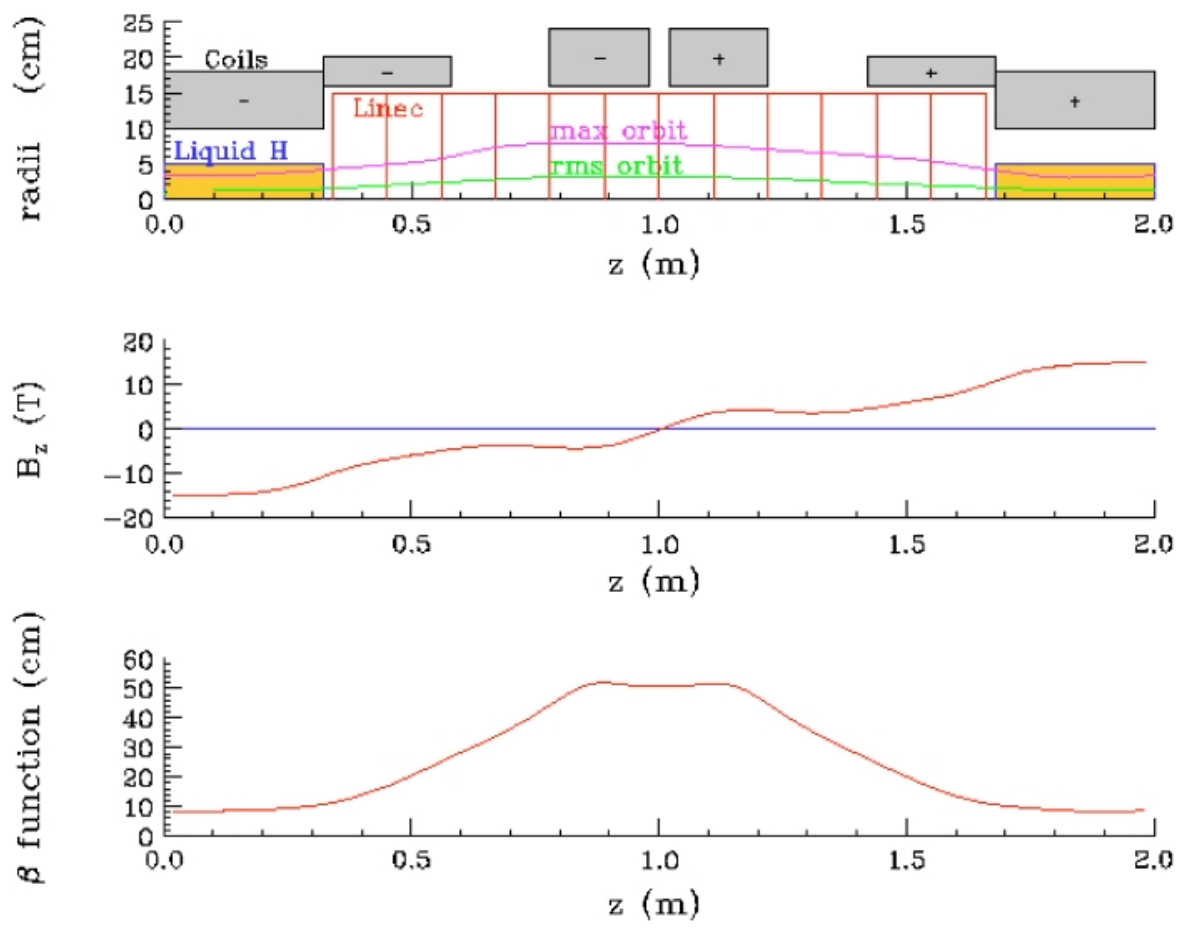

FIG. 24. (Color) (a) Cross section of one-half period of an alternating solenoid cooling lattice, (b) axial magnetic field vs $z$, and (c) $\beta_{\perp}$ function vs $z$. 


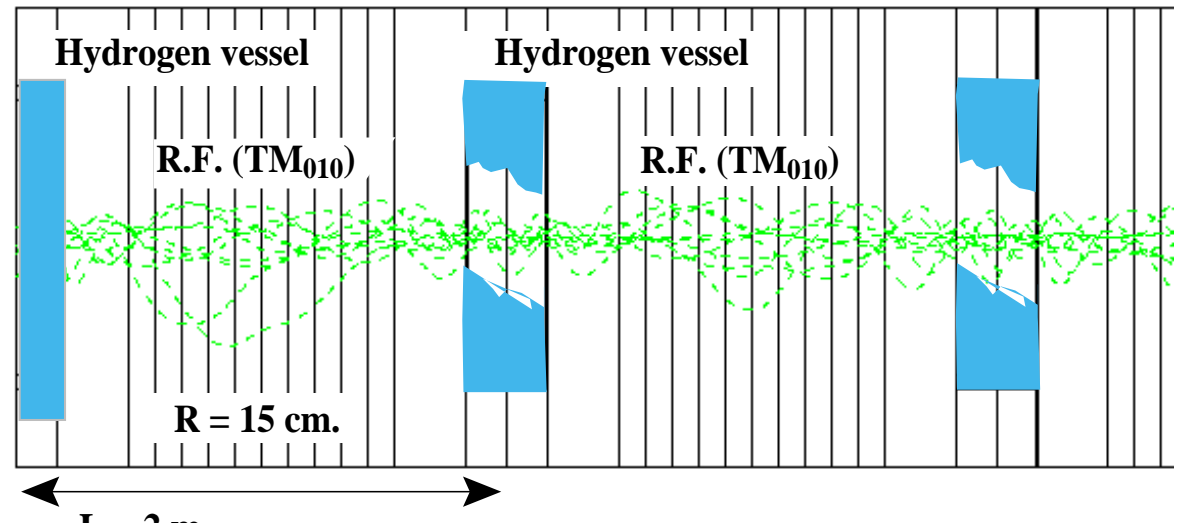

$\mathbf{L}=\mathbf{2} \mathbf{m}$.

FIG. 25. (Color) GEANT simulation of muons traversing a section of the alternating solenoid cooling channel. The variation of the magnetic field $B_{z}$ is shown for $1 \frac{1}{2}$ cells of the figure.

angular momentum. However, the presence of absorbers causes the canonical angular momentum to grow and would lead to severe emittance growth by the end of a long channel. This growth is stopped by alternating the direction of the solenoid field, as shown in Fig. 26(b). Simulations have shown that $2 \mathrm{~m}$ is a reasonable (half) period for the field, since the net growth in canonical angular momentum is small. In addition, synchrobetatron resonances are avoided since the periodicity of the field forces the average betatron wavelength to be $2 \mathrm{~m}$, whereas the synchrotron oscillation wavelength seen in the simulations for this arrangement is $\approx 14 \mathrm{~m}$.
Figure 27(a) shows the rms and maximum radius of any particle in the beam distribution as a function of distance along the channel. The rms radius shows that most of the beam is confined to within $2 \mathrm{~cm}$ of the axis. The peak rms radius decreases toward the end of the channel as a result of the cooling. The maximum particle radius is about $8 \mathrm{~cm}$, which determines the radius of the windows required in the rf cavities. Figure 27(b) shows the rms momentum spread corrected for the correlation between $p_{z}$ and transverse amplitude imposed on the initial particle distribution. The momentum spread grows as a function of distance since the alternating solenoid system cools


FIG. 26. (Color) (a) Average momentum vs $z$, (b) average angular momentum: mechanical (solid curve) and canonical (dashed curve) vs $z$. 

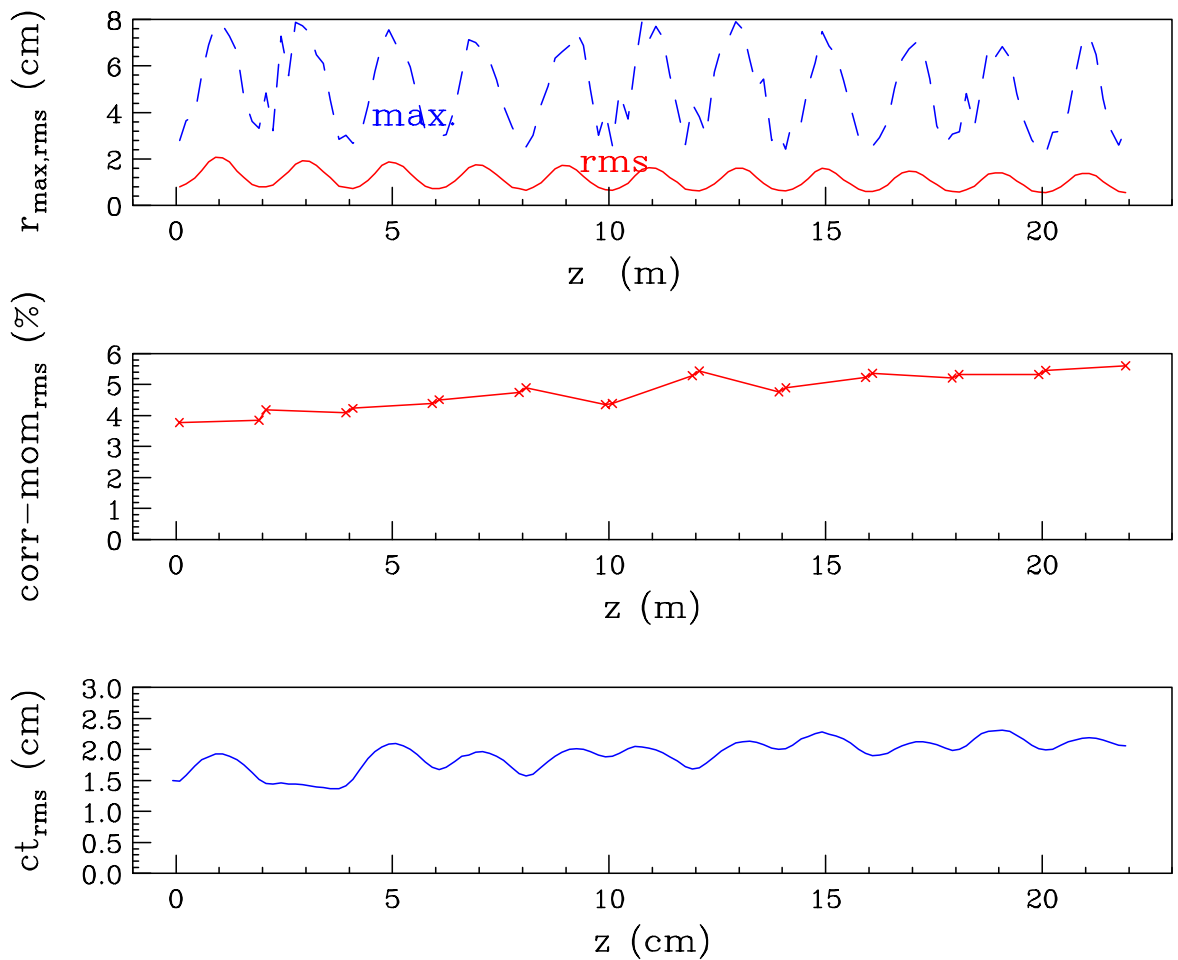

FIG. 27. (Color) (a) rms and maximum beam radii, (b) rms corrected momentum, and (c) rms bunch length, all vs $z$.

only the transverse emittance. Figure 27(c) shows the rms bunch length $s$ as a function of distance along the channel. Again, this grows with distance since this channel does not cool longitudinally.

Figure 28(a) shows the decrease in transverse normalized emittance as a function of distance along the channel. The system provides cooling by a factor of $\approx 2$ in both the $x$ and $y$ transverse phase spaces. From the changing slope of the curve we note that the rate of cooling is dropping. This sets $\approx 22 \mathrm{~m}$ as the maximum useful length for this type of system. It must be followed by a longitudinal emittance exchange region to reduce the momentum spread and bunch length approximately back to their starting values. Figure 28(b) shows the increase in longitudinal normalized emittance in the channel due to the increase in momentum spread and bunch length. Finally, Fig. 28(c) shows the decrease in the $6 \mathrm{D}$ normalized emittance as a function of distance along the channel. There is a net decrease in $6 \mathrm{D}$ emittance by a factor of $\approx 2$ in the channel. Table $\mathrm{V}$ gives the initial and final beam parameters.

This simulation has been confirmed, with minor differences, by double precision GEANT [152] and PARMELA [150] codes.

\section{E. 31 T solenoid transverse cooling example}

As in the preceding example, the lattice consists of 11 identical $2 \mathrm{~m}$ long cells with the direction of the fields in the solenoids alternating from one cell to the next. The maximum solenoidal field is higher $(31 \mathrm{~T})$ than in the previous example, but the bore can be smaller $(6<$ $r<8 \mathrm{~cm})$. Several hybrid magnets with at least this field have operated for many years [163]. More recent magnets are of even higher field. A hybrid solenoid with $45 \mathrm{~T}$ central field is under construction [164]. However, all of these magnets are of small bore and generate their rated field over a length of only a few centimeters. Also, typical hybrid magnet longevity is not adequate for our purposes. While $31 \mathrm{~T}$ magnets with the needed aperture appear practical, capital and operating costs are high. High-temperature superconductors are capable of adequate current densities for this field. The challenge is to make them in sufficient lengths and to circumvent their present engineering limitations. If the capital cost of magnets employing high $-T_{c}$ materials is not prohibitive, and solutions using lithium lenses are not chosen, then high-Tc magnets would be the preferred choice. Between the $31 \mathrm{~T}$ solenoids there are $1.3 \mathrm{~m}$ long matching sections with an inside diameter of $32 \mathrm{~cm}$, superimposed on a $36 \mathrm{MeV} / \mathrm{m}$ reacceleration linac operating at $805 \mathrm{MHz}$.

Table VI gives the initial and final parameters for the $31 \mathrm{~T}$ example, together with the required emittances for a Higgs factory. In setting these requirements a dilution of $20 \%$ during the acceleration is assumed in each of the three emittances.

\section{F. Bent solenoid emittance exchange example}

We have been considering using a system that exchanges longitudinal and transverse emittance by exploiting dispersion in a large acceptance channel, with a low- $Z$ wedge absorber in the region of dispersion. 

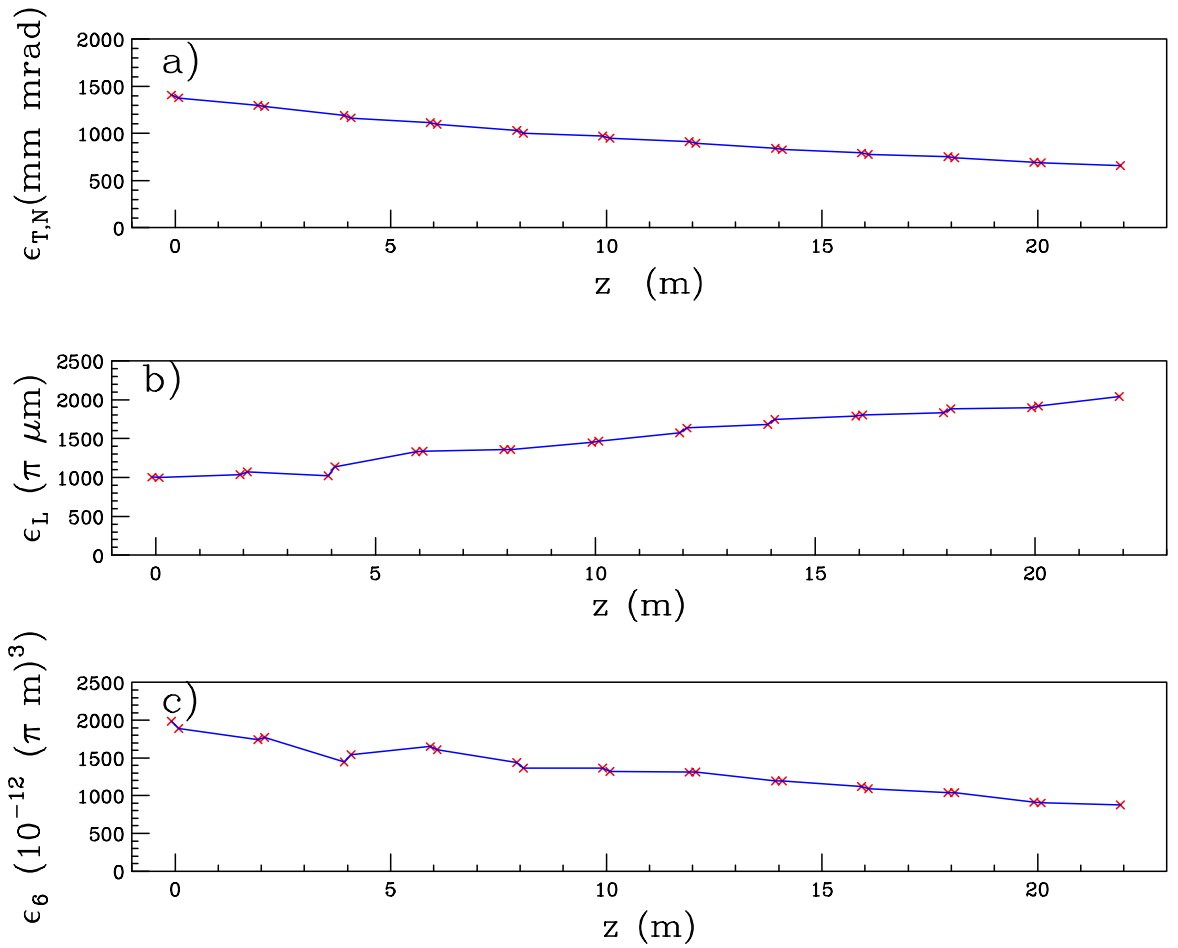

FIG. 28. (Color) Emittance vs $z$ : (a) transverse emittance $\epsilon_{T, N}(\pi \mathrm{mm} \mathrm{mrad})$, (b) longitudinal emittance $\epsilon_{L}\left(10^{-3} \pi \mathrm{mm}\right)$, and (c) $6 \mathrm{D}$ emittance $\epsilon_{6}\left(10^{-12} \pi \mathrm{m}^{3}\right)$.

In a bent solenoid, in the absence of any dipole field, there is a drift perpendicular to the bend plane of the center of the Larmor circular orbit, which is proportional to the particle's momentum $[165,166]$. In our example we have introduced a uniform dipole field over the bend to cancel this drift exactly for particles with the reference momentum. Particles with momenta differing from the reference momentum then spread out spatially, giving the required dispersion $(0.4 \mathrm{~m})$. The momentum spread is reduced by introducing liquid hydrogen wedges [167]. The hydrogen wedges would be contained by thin beryllium or aluminum foils, but these were not included in this simulation.

After one bend and one set of wedges, the beam is asymmetric in cross section. Symmetry is restored by a following bend and wedge system rotated by $90^{\circ}$ with respect to the first. Figure 29 shows a representation of the two bends and wedges. The total solenoid length was $8.5 \mathrm{~m}$. The beam tube outside diameter is $20 \mathrm{~cm}$, and the minimum bend radii is $34 \mathrm{~cm}$.

Figure 30(a) shows the magnetic fields $\left(B_{z}, B_{y}\right.$, and $\left.B_{x}\right)$ as a function of the position along the cell. The solenoid bend curvature is exactly that given by the trajectory of a reference particle [equal in momentum to the average momenta given in Fig. 30(b)] in the given transverse fields. The actual shape of the bend turns out to be very important. Discontinuities in the bend radius can excite perturbations which increase the transverse emittance. We have shown, for example, that the transverse emittance growth in a bent solenoid depends on discontinuities of the bend radius as

TABLE V. Initial and final beam parameters in a $15 \mathrm{~T}$ transverse cooling section.

\begin{tabular}{lccc}
\hline \hline & Initial & Final & Final/Initial \\
\hline Particles tracked & 1000 & 980 & 0.98 \\
Reference momentum $(\mathrm{MeV} / c)$ & 186 & 186 & 1.0 \\
Transverse emittance $(\pi \mathrm{mm} \mathrm{mrad})$ & 1400 & 600 & 0.43 \\
Longitudinal emittance $(\pi \mathrm{mm})$ & 1.1 & 2.3 & 2.09 \\
6D emittance $\left(\times 10^{-12}\right)(\pi \mathrm{m} \mathrm{rad})^{3}$ & 2000 & 800 & 0.40 \\
rms beam radius in hydrogen $(\mathrm{cm})$ & 0.8 & 0.55 & 0.69 \\
rms beam radius in linac $(\mathrm{cm})$ & 2.0 & 1.4 & 0.70 \\
max beam radius in linac $(\mathrm{cm})$ & 7.0 & 7.0 & 1.0 \\
rms bunch length $(\mathrm{cm})$ & 1.5 & 2.2 & 1.5 \\
max bunch full width $(\mathrm{cm})$ & 13 & 19 & 1.5 \\
rms $\Delta p / p(\%)$ & 3.8 & 5.6 & 1.5 \\
\hline \hline
\end{tabular}


TABLE VI. Initial and final beam parameters in a $31 \mathrm{~T}$ transverse cooling section.

\begin{tabular}{lccc}
\hline \hline & Initial & Final & Final/Initial \\
\hline Particles tracked & 4000 & 3984 & 0.99 \\
Reference momentum $(\mathrm{MeV} / c)$ & 186 & 186 & 0.52 \\
Transverse emittance $(\pi \mathrm{mm} \mathrm{mrad})$ & 460 & 240 & 1.9 \\
Longitudinal emittance $(\pi \mathrm{mm})$ & 0.85 & 1.6 & 0.63 \\
6D emittance $\left(\times 10^{-12}\right)(\pi \mathrm{m} \mathrm{rad})^{3}$ & 150 & 95 & 0.75 \\
rms beam radius in hydrogen $(\mathrm{cm})$ & 0.44 & 0.33 & 0.80 \\
rms beam radius in linac $(\mathrm{cm})$ & 0.4 & 1.1 & 1.0 \\
max beam radius in linac $(\mathrm{cm})$ & 6.0 & 6.0 & 1.2 \\
rms bunch length $(\mathrm{cm})$ & 1.5 & 1.8 & 1.7 \\
max bunch full width $(\mathrm{cm})$ & 11 & 19 & 1.4 \\
rms $\Delta p / p(\%)$ & 3.5 & 5.0 & 98 \\
\hline \hline
\end{tabular}

a function of distance, and its first and second derivatives, the size and tilt of the solenoidal coils, auxiliary fields, and the $6 \mathrm{D}$ phase space of the beam. Thus optimization is not straightforward. One solution to this problem is to have long, adiabatic bends. However, this adds undesirable length to the emittance exchange section. We are studying options with coupling sections to tight bends roughly half a Larmor length long, which seems to minimize transverse emittance growth while also minimizing the length of the section. Due to similar problems, the length and longitudinal distribution of the wedge material has also been found to affect emittance growth. For example, the growth can be minimized when the vector sum of the Larmor phases at the absorber elements is small or zero.

The simulations were performed using the program ICOOL. The maximum beam radius is $10 \mathrm{~cm}$. Transmission was $100 \%$. Figure 31(a) shows the rms longitudinal momentum spread relative to the reference momentum as a function of the position along the cell. The fractional spread decreases from an initial value of approximately $5 \%$ to a final value of approximately $2.2 \%$. At the same time, since this is an emittance exchange, the transverse beam area grows, as shown in Fig. 31(b). One notes that



FIG. 29. (Color) Representation of a bent solenoid longitudinal emittance exchange section. the area increases not only in the regions of bends (regions 1 and 8), but also in the regions of wedges (regions 2-6 and 9-11). This is probably due to failures in betatron matching that have yet to be understood.

Figure 32 shows scatter plots of the transverse particle positions against their momenta. The dispersion is clearly observed in Fig. 32(b) (after the first bend) and in Fig. 32(e) (after the second bend). It is seen to be removed, with a corresponding decrease in momentum spread, in Fig. 32(c) (after the first set of wedges) and Fig. 32(f) (after the second set of wedges).

Figure 33 shows a scatterplot of the square of the particle radii vs their longitudinal momenta, (a) at the start and (b) at the end of the emittance exchange section. The decrease in momentum spread and rise in beam area are clearly evident.

The initial and final beam parameters are given in Table VII. Although this example demonstrates a factor of $\approx 3$ reduction in the longitudinal momentum spread, there is a $37 \%$ increase in the $5 \mathrm{D}$ phase space. The simulations must be extended to include rf so that the $6 \mathrm{D}$ emittance can be studied and the emittance exchange section can be optimized.

Emittance exchange in solid $\mathrm{LiH}$ wedges, with ideal dispersion and matching, has also been successfully simulated using SIMUCOOL [168]. Dispersion generation by weak focusing spectrometers [159] and dipoles with solenoids [169] have also been studied.

\section{G. rf for the cooling systems}

The losses in the longitudinal momentum of the muon beam from the cooling media have to be restored using $\mathrm{rf}$ acceleration sections. These rf structures are embedded in solenoidal fields that reverse direction within each section. In the two transverse cooling examples above, the $\mathrm{rf}$ frequency is $805 \mathrm{MHz}$ and the peak gradient is $36 \mathrm{MeV} / \mathrm{m}$. The magnetic fields that extend over the cavities vary from 0 to $10 \mathrm{~T}$, reversing in the center. It should be pointed out that in the earlier stages, the bunches are longer, and lower frequencies will be required. 



FIG. 30. (Color) (a) Axial $B_{z}$ and dipole $B_{y}, B_{x}$, magnetic fields, and (b) average momentum, both as a function of the position along the cell.

In order to realize maximum accelerating gradients within the acceleration cavities, we take advantage of the penetrating properties of a muon beam by placing thin windows between each $\mathrm{rf}$ cell, thereby creating an accelerating structure closely approximating the classic pill-box cavity. This permits operating conditions in which the axial accelerating field is equal to the maximum wall field and gives a high shunt impedance.
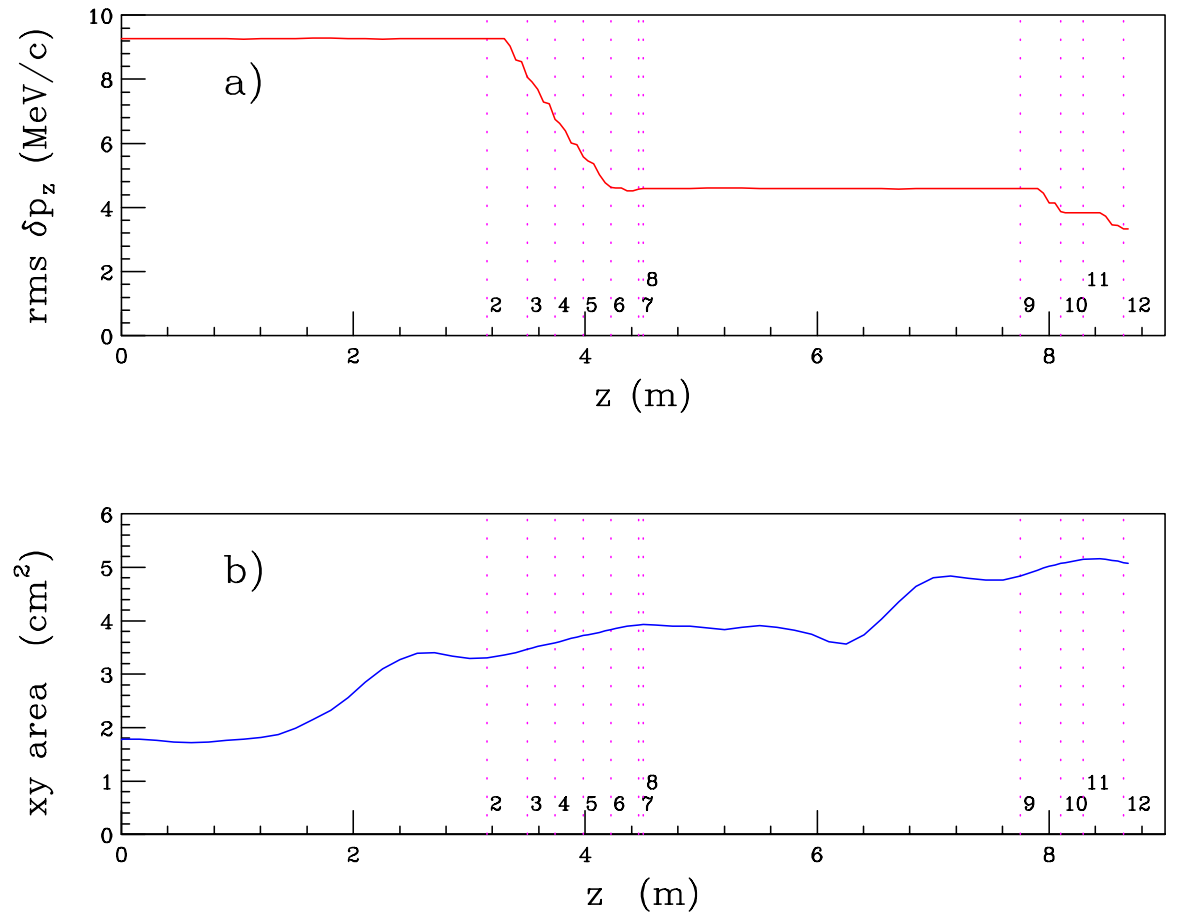

FIG. 31. (Color) (a) rms longitudinal $\delta p_{z}$ with respect to the reference momentum and (b) transverse beam area, both as a function of $z$. 

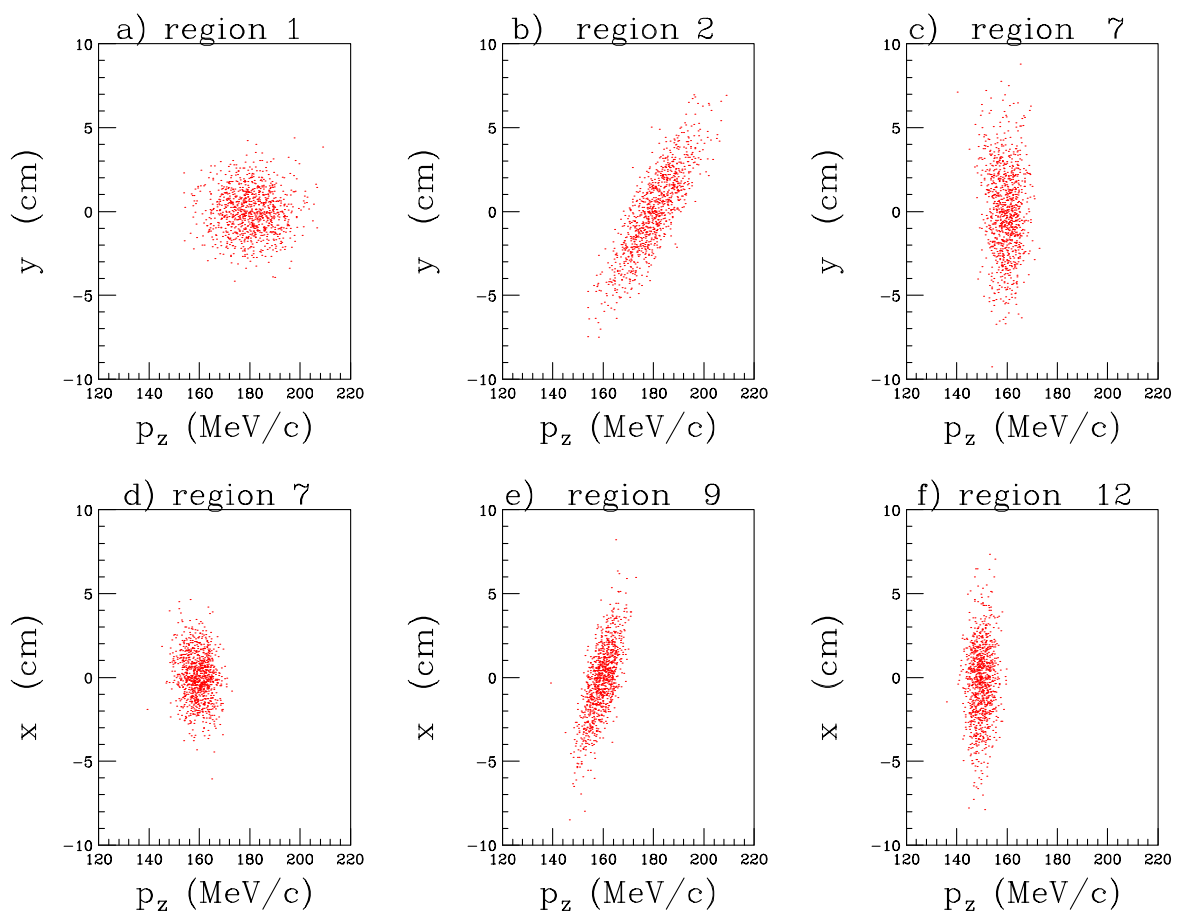

FIG. 32. (Color) $y$ vs $p_{z}$ plots: (a) at the start, (b) after the first bend, and (c) after the first set of wedges. $x$ vs $p_{z}$ plots: (d) after the first wedges, (e) after the second bend, and (f) at the end of the emittance exchange section, following the second set of wedges.

The windows in the $15 \mathrm{~T}$ example are $16 \mathrm{~cm}$ diameter, $125 \mu \mathrm{m}$ thick Be foils. In the $31 \mathrm{~T}$ case, they are $10 \mathrm{~cm}$ diameter and $50 \mu \mathrm{m}$ thick. Two studies indicate that at nitrogen temperature the Ohmic losses at the foils are negligible and the mechanical deformation is tolerable [170].

For these rf structures, we will use an interleaved cavity design in which two parts are independently powered
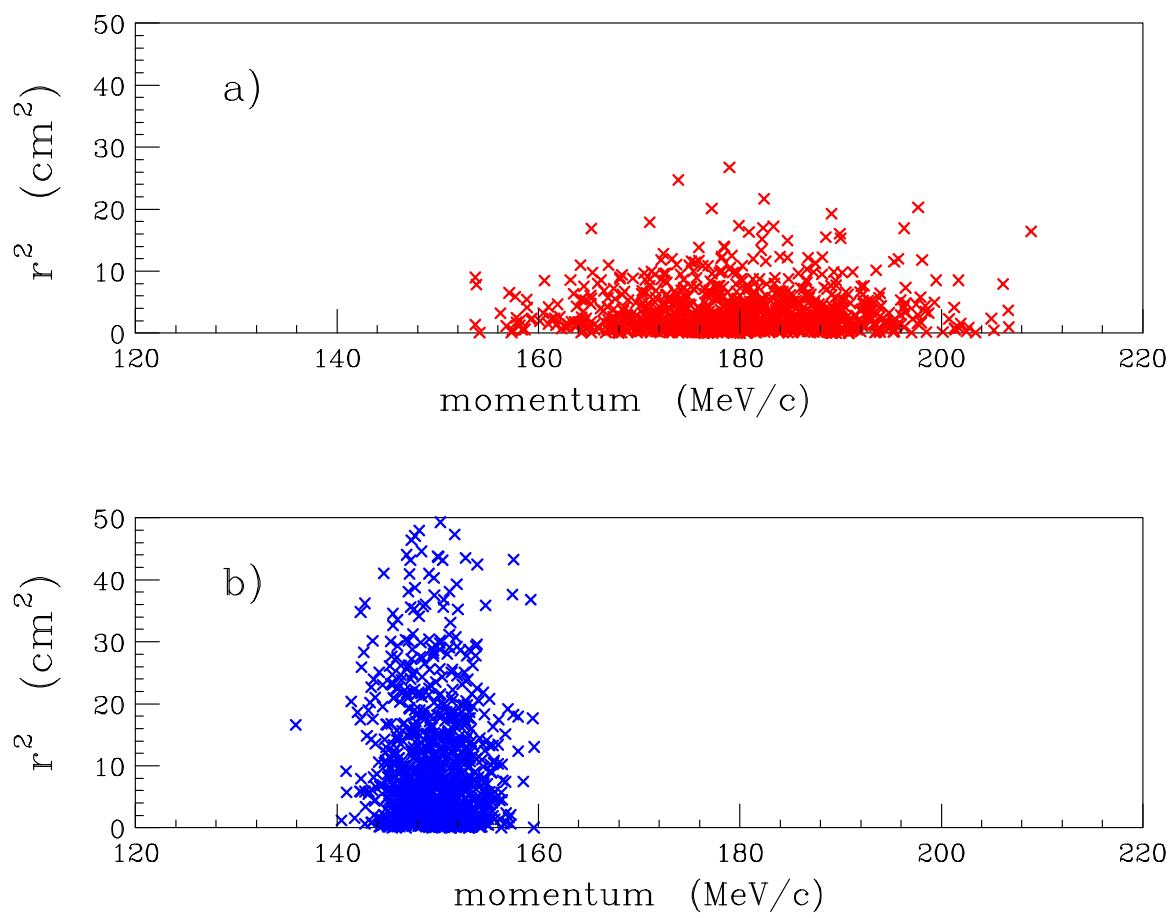

FIG. 33. (Color) Scatter plot of squared radii vs longitudinal momentum: (a) at the start and (b) at the end of the emittance exchange section. 
TABLE VII. Initial and final beam parameters in a longitudinal emittance exchange section.

\begin{tabular}{lccc}
\hline \hline & Initial & Final & Final/Initial \\
\hline Longitudinal momentum spread $(\mathrm{MeV} / c)$ & 9.26 & 3.35 & 0.36 \\
Ave. momentum $(\mathrm{MeV} / c)$ & 180 & 150 & 0.83 \\
Transverse size $(\mathrm{cm})$ & 1.33 & 2.26 & 1.70 \\
Transverse momentum spread $(\mathrm{MeV} / c)$ & 6.84 & 7.84 & 1.15 \\
Transverse emittance $(\pi \mathrm{mm} \mathrm{mrad})$ & 870 & 1694 & 1.95 \\
Emit $_{\text {trans }}^{2} \times \Delta p_{\text {long }}\left[(\pi \mathrm{m} \mathrm{mrad})^{2} \mathrm{MeV} / c\right]$ & 7.0 & 9.6 & 1.37 \\
\hline \hline
\end{tabular}

(Fig. 34). The mode of the system will be referred to as $\pi / 2$ interleaved. Each section supports a standing wave $\pi$ mode, with each acceleration cell $\pi / 2$ long, giving a good transit time factor. To reduce the peak rf power requirements (by a factor of 2), we are considering operating the cells at liquid nitrogen temperatures.

The characteristics of the rf systems currently being studied are summarized in Table VIII. Figure 35 shows a full $1.3 \mathrm{~m}$ section with interleaved cavities. Each cell is $8.1 \mathrm{~cm}$ in length and the $1.3 \mathrm{~m}$ section consists of 16 cells.

\section{H. The liquid lithium lens}

The final cooling element ultimately determines the luminosity of the collider. In order to obtain smaller transverse emittance as the muon beam travels down the cooling channel, the focusing strength must increase, i.e., the $\beta_{\perp}$ 's must decrease. A current within a conductor produces an active lens absorber, which can maintain the beam at small $\beta_{\perp}$ throughout an extended absorber length, while simultaneously attenuating the beam momentum. An active lens absorber, such as a lithium lens, may prove to be the most efficient cooling element for the final stages.

The cooling power of a Li lens is illustrated in Fig. 36, where the $x$ vs $p_{x}$ phase space distributions at the begin-

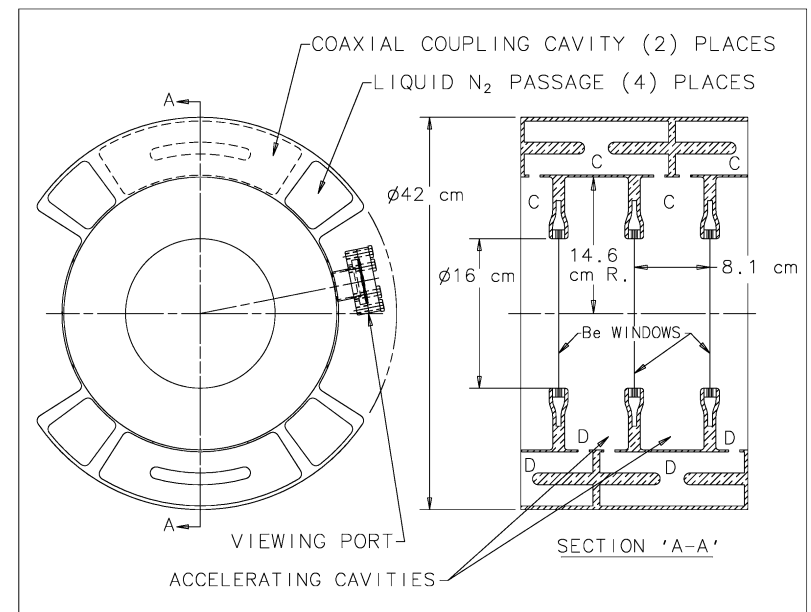

FIG. 34. Two full cell sections plus two half-cell sections of the interleaved $\pi / 2$ mode accelerating cavities. The volumes labeled $\mathrm{C}$ are powered separately from the volumes labeled D. ning and end of the absorber are shown. This example corresponds to a $1 \mathrm{~m}$ long lens, with $1 \mathrm{~cm}$ radius, and a surface field of $10 \mathrm{~T}$. The beam momentum entering the lens was $267 \mathrm{MeV} / c$, with Gaussian transverse spatial and momentum distributions $\sigma_{x}=\sigma_{y}=2.89 \mathrm{~mm}$, $\sigma_{p_{x}}=\sigma_{p_{y}}=26.7 \mathrm{MeV} / c$, and a normalized emittance of $\epsilon_{x, N}=710 \mathrm{~mm} \mathrm{mrad}$. The normalized emittance at the end of the absorber was $\epsilon_{x, N}=450 \mathrm{~mm}$ mrad (cooling factor $\sim 1.57$ ), and the final beam momentum was $159 \mathrm{MeV} / c$. The results were obtained using a detailed GEANT simulation of a single stage.

An alternative cooling scheme under study uses a series of Li lenses. The lens parameters would have to vary to match the changing beam emittance along the section, and, in addition, acceleration of the beam between the lenses has to be included.

Lithium lenses have been used with high reliability as focusing elements at FNAL and CERN [7,156,157]. Although these lenses have many similar properties to those required for ionization cooling, there are some very crucial differences which will require significant advances in lens technology: ionization cooling requires longer lenses $(\sim 1 \mathrm{~m})$, higher fields $(\sim 10 \mathrm{~T})$, and higher operation rates $(15 \mathrm{~Hz})$. The last requirement calls for operating the lenses with lithium in the liquid phase. A liquid Li lens consists of a small diameter rodlike chamber filled with liquid Li through which a large current is drawn.

The azimuthal magnetic field focuses the beam to give the minimum achievable emittance $\epsilon_{x, N} \approx C \beta_{\perp}$, where

TABLE VIII. Characteristics of the rf system.

\begin{tabular}{lc}
\hline \hline rf frequency $(\mathrm{MHz})$ & 805 \\
Cavity length $(\mathrm{cm})$ & 8.1 \\
Cavity inner radius $(\mathrm{cm})$ & 14.6 \\
Cavity outer radius $(\mathrm{cm})$ & 21 \\
$Q / 1000$ & $2 \times 20$ \\
Peak axial gradient $(\mathrm{MV} / \mathrm{m})$ & 36 \\
Shunt impedance $(\mathrm{M} \Omega / \mathrm{m})$ & $2 \times 44$ \\
$Z t^{2}(\mathrm{M} \Omega / \mathrm{m})$ & $2 \times 36$ \\
Fill time $(3 \tau)(\mu \mathrm{s})$ & $2 \times 12$ \\
rf peak power $(\mathrm{MW} / \mathrm{m})$ & $\frac{1}{2} \times 29$ \\
Ave. power $(15 \mathrm{~Hz})(\mathrm{KW} / \mathrm{m})$ & 5.3 \\
Be window aperture $(\mathrm{cm})$ & $16(10$ for $31 \mathrm{~T}$ case $)$ \\
Be window thickness $(\mu \mathrm{m})$ & $127(50$ for $31 \mathrm{~T}$ case $)$ \\
\hline
\end{tabular}




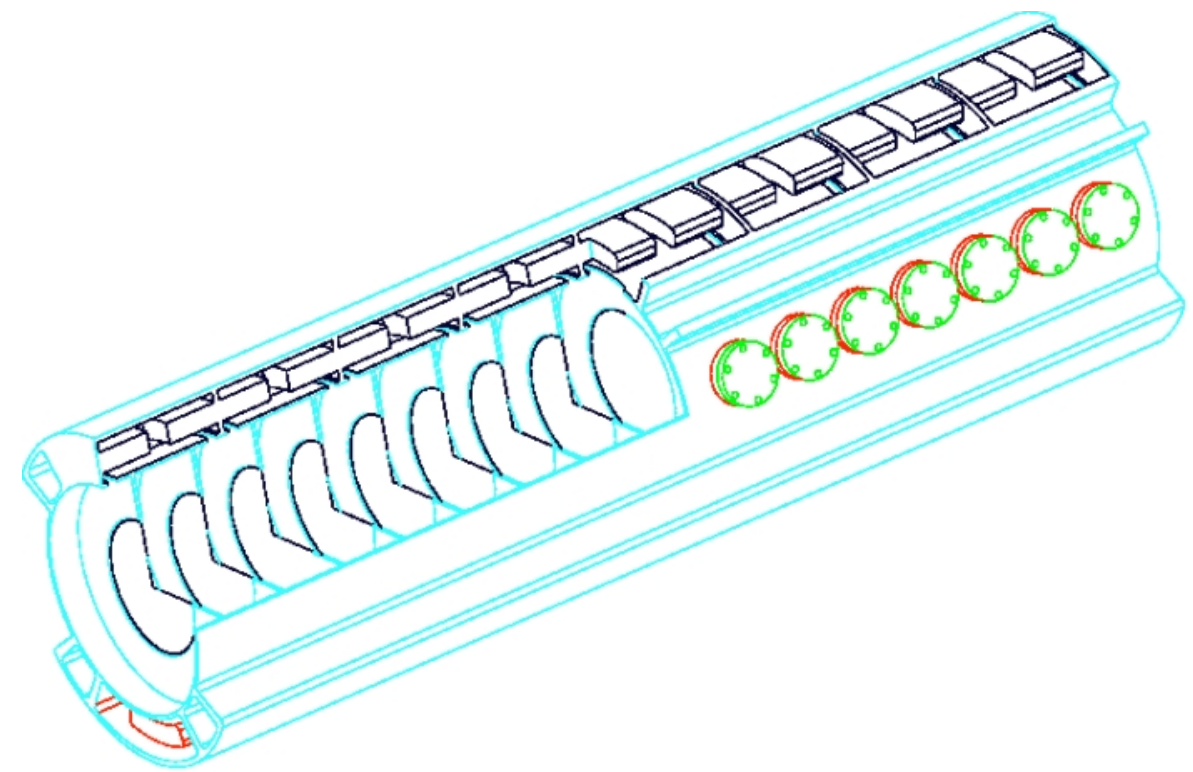

FIG. 35. (Color) A $1.3 \mathrm{~m}$ acceleration section with quarter section cut away for viewing the intercavity windows.

the constant $C$ depends on the properties of the material, for example, $C_{L i}=79 \mathrm{~mm} \mathrm{mrad} / \mathrm{cm}$. The focusing term can be written as $\beta_{\perp} \sim 0.08(\mathrm{~cm}) \sqrt{p / J}$ with $p$ the muon momentum in $\mathrm{MeV} / c$, and $J$ the current density in $\mathrm{MA} / \mathrm{cm}^{2}$. Increasing $J$ is obviously desirable. Decreasing $p$ can also be useful. However, below about $250 \mathrm{MeV} / c$ the slope of $\frac{d E}{d x}(E)$ tends to increase the longitudinal emittance. The requirement for the highest current density causes large Ohmic power deposition. The current den-
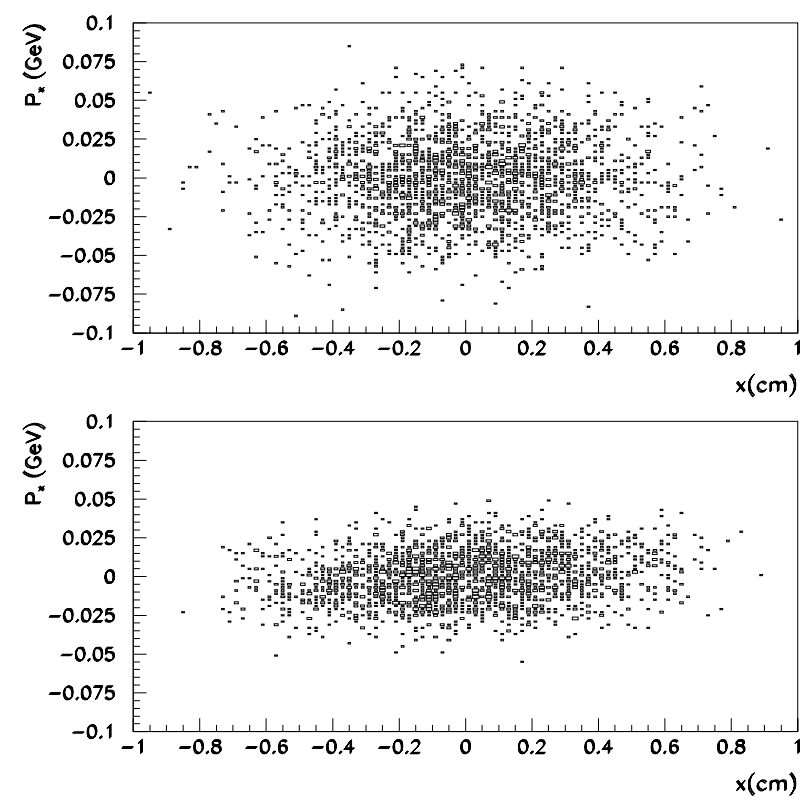

FIG. 36. $x-p_{x}$ phase space distribution at the beginning (top) and at the end (bottom) of the absorber described in the text. sity will be limited by the maximum tolerable deposited energy, which will produce instantaneous heating, expansion, and pressure effects. Understanding these effects is part of the ongoing liquid $\mathrm{Li}$ lens R\&D.

The structural design of the lithium lens is determined by how the pressure pulse and heat deposition are handled. We assume that the Li will be flowing rapidly under high pressure, confined by electrical insulators radially and by fairly thick Be windows longitudinally. Operation at $15 \mathrm{~Hz}$ for long periods poses severe challenges. Shock, fatigue, and other failure modes are being evaluated, in addition to studies of material compatibilities, corrosion, and degradation to ensure safe operation over long periods. It seems that the minimum required radius of the lens may be the most important parameter to determine, since mechanical problems increase while losses decrease as a function of radius.

Transferring the beam from one lens to another, with linacs to reaccelerate and provide longitudinal focusing, is also a challenging problem, because of the multiple scattering introduced in the windows, straggling, and the large divergence of the beams. We are in the process of evaluating a number of designs for this transfer channel, using detailed tracking simulations that include solenoids, quadrupoles, and other focusing elements together with $\mathrm{Li}$ lenses.

A group from BINP has designed, and is constructing, a $15 \mathrm{~cm}$ long liquid lithium lens prototype that will eventually be tested at FNAL. It is planned to extend this R\&D program to design, construct, and test longer lenses. The design of two lenses, whose behavior will be tested at first on a bench and then with muon beams at the Ionization Cooling Demonstration Facility, will then follow [171]. 


\section{Ionization cooling experimental R\&D}

An $R \& D$ program has been proposed to design and prototype the critical sections of a muon ionization cooling channel. The goal of this experimental R\&D program is to develop the muon ionization cooling hardware to the point where a complete ionization cooling channel can be confidently designed for the first muon collider. Details can be found in the Fermilab proposal P904 [171]. A summary of the R\&D program can be found in Ref. [172].

The proposed R\&D program consists of:

(i) Developing an appropriate rf reacceleration structure. It is proposed to construct a three-cell prototype $\mathrm{rf}$ cavity with thin beryllium windows, which will be tested at high power and within a high-field solenoid.

(ii) Prototyping initially a $2 \mathrm{~m}$ section, and eventually a $10 \mathrm{~m}$ section, of an alternating solenoid transverse cooling stage. It is proposed to test the performance of these sections in a muon beam of the appropriate momentum.

(iii) Prototyping an emittance exchange (wedge) section and measuring its performance in a muon beam of the appropriate momentum.

(iv) Prototyping and bench testing $\sim 1 \mathrm{~m}$ long liquid lithium lenses, and developing lenses with the highest achievable surface fields, and hence the maximum radial focusing. (v) Prototyping a lithium lens-rf-lens system and measuring its performance in a muon beam of the appropriate momentum.

(vi) Developing, prototyping, and testing a hybrid lithium lens/wedge cooling system.

The measurements that are needed to demonstrate the cooling capability and optimize the design of the alternating solenoid, wedge, and lithium lens cooling stages will require the construction and operation of an ionization cooling test facility. This facility will need (i) a muon beam with a central momentum that can be chosen in the range $100-300 \mathrm{MeV} / c$, (ii) an experimental area that can accommodate a cooling and instrumentation setup of initially $\sim 30 \mathrm{~m}$ in length, and eventually up to $\sim 50 \mathrm{~m}$ in length, and (iii) instrumentation to precisely measure the positions of the incoming and outgoing particles in $6 \mathrm{D}$ phase space and confirm that they are muons.

In the initial design shown in Fig. 37, the instrumentation consists of identical measuring systems before and after the cooling apparatus [173]. Each measuring system consists of (a) an upstream time measuring device to determine the arrival time of the particles to one-quarter of an rf cycle ( $\sim 300 \mathrm{ps})$, (b) an upstream momentum spectrometer in which the track trajectories are measured by low pressure TPC's on either side of a bent solenoid, (c) an accelerating rf cavity to change the particle's momentum by an amount that depends on its arrival time,

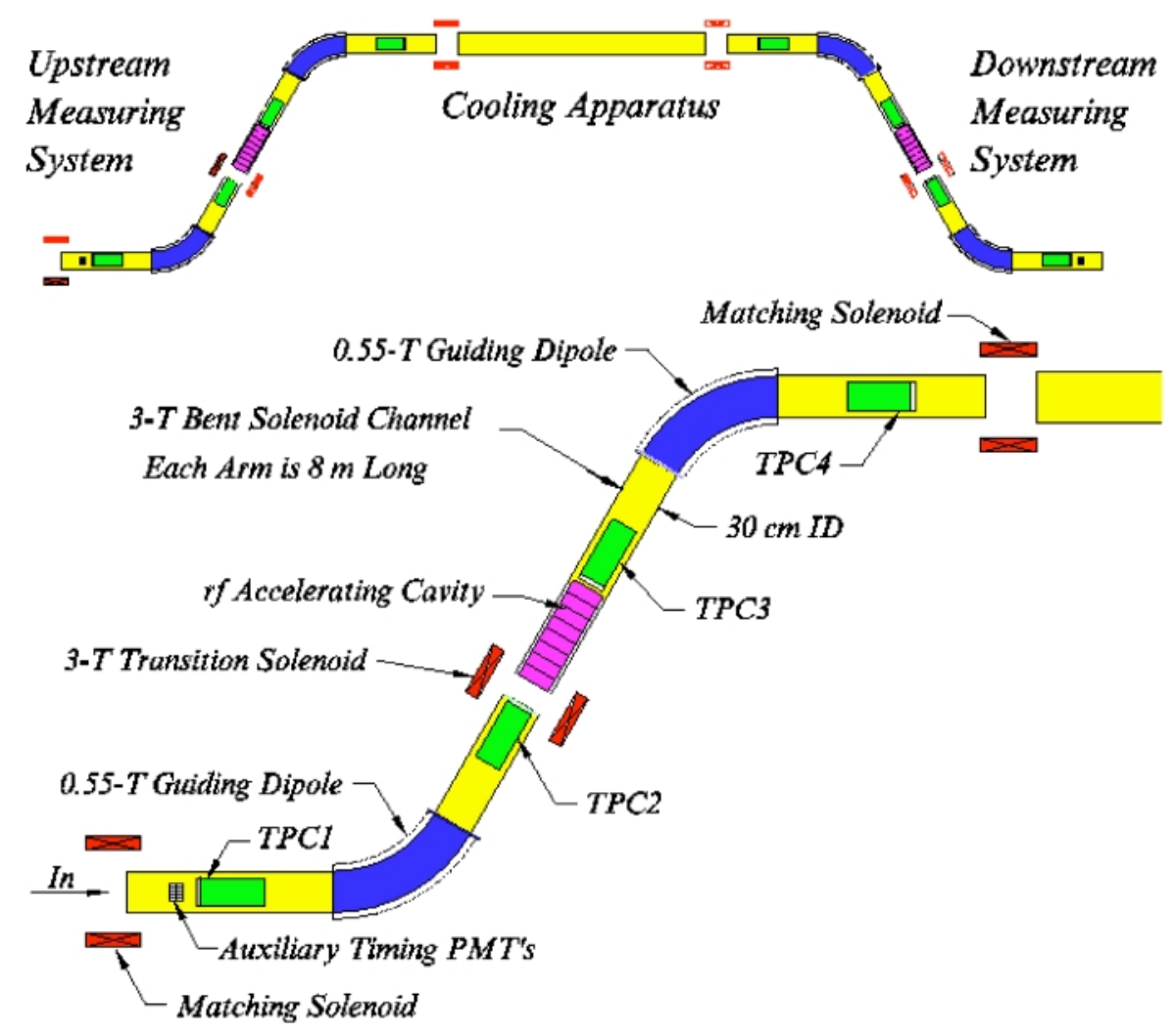

FIG. 37. (Color) Schematic of the cooling test apparatus arrangement. 
and (d) a downstream momentum spectrometer, which is identical to the upstream spectrometer, and together with the rf cavity and the upstream spectrometer forms a precise time measurement system with a precision of a few ps. The measuring systems are $8 \mathrm{~m}$ long, and are contained within a high-field solenoidal channel to keep the beam particles within the acceptance of the cooling apparatus.

It is proposed to accomplish this ionization cooling $R \& D$ program in a period of about six years. At the end of this time we believe that it will be possible to assess the feasibility and cost of constructing an ionization cooling channel for the first muon collider, and, if it proves feasible, begin a detailed design of the complete cooling channel.

\section{ACCELERATION}

\section{A. Introduction}

Following cooling and initial bunch compression, the beams must be rapidly accelerated. In this section some of the options in accelerator design will be described and examples of acceleration scenarios presented.

Separate acceleration scenarios are given here for a low momentum spread $100 \mathrm{GeV}$ first muon collider (Higgs factory), and for a high luminosity $3 \mathrm{TeV}$ collider. Ideally, though more difficult, this accelerator designed for the low energy machine should be extendable to the $\approx 250 \mathrm{GeV}$ beam energy and from there to the $\approx 2 \mathrm{TeV}$ beam energy needed for a very high energy collider.

While acceleration of muons to high energy is clearly possible, an optimal and cost-effective acceleration complex is needed. In the scenarios described below, a low-frequency linac would take the beam from the end of cooling to an energy of $\approx 1 \mathrm{GeV}$ followed by recirculatinglinac systems to take the beam to $50-70 \mathrm{GeV}$. The multi$\mathrm{TeV}$ energy regime can be reached through a series of very rapid cycling synchrotrons. Variations on the acceleration model and potential difficulties are discussed, including the use of FFAG accelerators in place of, or together with, the recirculating linacs. Finally, topics for further study and research are described.

\section{B. Accelerator options}

The acceleration time is limited by muon decay $\left(\tau_{\mu}=\right.$ $2.2 \mu \mathrm{s}$ at rest) and requires that

$$
e V_{\mathrm{rf}}^{\prime} \gg 0.16 \mathrm{MeV} / \mathrm{m} \text {, }
$$

where $e V_{\mathrm{rf}}^{\prime}$ is the acceleration rate. An acceleration rate value of $0.16 \mathrm{MeV} / \mathrm{m}$ is low for a linac, but very high for a conventional synchrotron.

At the lowest energies $(<700 \mathrm{MeV})$, the momentum spread and beam sizes are so large that only a linac is feasible, and acceleration to full energy in a single-pass linac would be good, but it would be very expensive.

Thus, following the initial linac, some form of recirculating acceleration is preferred. A synchrotron would be possible, in principle, but the acceleration must occur so rapidly that conventional magnet ramping is unlikely to be practical. Two alternative multipass methods are being considered: recirculating linac accelerators similar to those used at the Thomas Jefferson National Accelerator Facility (TJNAF) and FFAG accelerators.

In a recirculating linac accelerator, the beam is circulated through the same linac for several passes, with separate, energy matched, fixed-field return paths for each pass. Each return path is optically independent and can be separately designed. In the initial lattice design for the muon recirculating linac accelerator, the return arcs are similar alternating gradient (AG) systems with the same dipole layout, but with differing quadrupole strengths to allow separate tuning and chronicity matching in each arc. Multiple aperture superconducting magnets have also been designed which would reduce the diameter of the recirculating linac, lowering muon loss from decay and possibly being more economical (see Fig. 7.12 of Ref. [44]). In either case, both the linac and return transports must accommodate large transverse emittances (rms) of $\approx 300 \pi \mathrm{mm} \mathrm{mrad}$. Strong focusing is required not only to keep apertures down, but also to minimize orbit deviations due to the large momentum spreads, which in the initial stages of acceleration can be as large as $10 \%$ rms.

More recently, an adaptation of the FFAG accelerator concept has been proposed for $\mu^{+} \mu^{-}$acceleration $[174,175]$. In this variation, return transports are designed with a very large (factor of 5-10) energy acceptance, so that separate energy turns can pass through the same fixed-field elements. More acceleration turns are possible than with a recirculating linac accelerator which reduces the rf requirements; but the orbits and focusing properties are now energy dependent. Such FFAG configurations require strong superconducting magnets with large apertures to accommodate the energy-dependent spread in closed orbits. The extra cost associated with increased magnet apertures must be evaluated against potential savings in the number of magnets and reduced $\mathrm{rf} /$ turn requirements.

For the higher energy stages, the muon lifetime is greater and the needed rate of energy increase is less. Thus, above a few hundred $\mathrm{GeV}$, rapid accelerating synchrotrons become possible. In a rapid accelerating synchrotron, the beam is also multipass accelerated through an rf system, but the beam returns in a single arc, as the magnetic field is ramped to match the increase in beam energy. As above, more acceleration turns are possible than with a recirculating accelerator, but we now have a single moderate aperture return transport.

Thus a complete system would likely include an initial linac followed by a sequence of recirculating 
linac accelerators and/or fixed field alternating gradient machines. Depending on the final collider energy, one or more rapid acceleration synchrotrons would follow. Each system increases the beam energy by a factor of 5-10.

\section{Scenario examples}

Several scenarios were discussed earlier [176,177]; see for instance the parameters (Table XI) used in a simulation of longitudinal motion discussed below. The ones given here are more recent, and more detailed, but they should not be taken to be definitive. They are examples that were derived to probe the design problems and to show that solutions should be possible.

\section{Acceleration for Higgs collider}

Table IX gives an example of a sequence of accelerators for a $100 \mathrm{GeV}$ Higgs factory, i.e., a machine with very low momentum spread $(0.003 \%$; see Table I) and relatively large rms transverse emittance $(\approx 300 \pi \mathrm{mm} \mathrm{mrad})$.

Following initial linacs, recirculating accelerators are used. The number of arcs in each recirculating accelerator is about 10. In this example, conventional fixed field $2 \mathrm{~T}$ magnets are used, but the effective ramp frequencies that would be needed if pulsed magnets were used are given for reference.

In this example, all the accelerating cavities are room temperature copper structures, and the accelerating gradients are modest $(<10 \mathrm{MeV} / \mathrm{m})$. Nevertheless, the acceleration is rapid enough that the total losses from decay are only $30 \%$. The heating from these decays is also modest $(\approx 10 \mathrm{~W} / \mathrm{m})$ because of the small number of turns and relatively low energy. Since no superconducting magnets or rf are used in this example, this heating should cause no problem.

In this machine, the transverse emittances are large and strong focusing is thus required, but the maximum momentum spread is moderate (up to $1.37 \% \mathrm{rms}$ in the first recirculator) and is thus not likely to be a problem.

If the same machine is also to run at a high luminosity, with larger momentum spread, then, although the $6 \mathrm{D}$ emittance is the same as in the Higgs collider discussed above, the transverse emittance is smaller $(\approx 90 \pi \mathrm{mmmrad} \mathrm{rms})$ and the longitudinal emittance larger (by about a factor of 4), and the momentum

TABLE IX. Accelerator parameters for a Higgs factory $(100 \mathrm{GeV})$.

\begin{tabular}{|c|c|c|c|c|c|c|}
\hline $\begin{array}{l}\text { Acc. type } \\
\text { Magnet type } \\
\text { rf type }\end{array}$ & $\begin{array}{c}\text { Linac } \\
\mathrm{Cu} \\
\end{array}$ & $\begin{array}{c}\text { Linac } \\
\mathrm{Cu} \\
\end{array}$ & $\begin{array}{c}\text { Recirc. } \\
\text { Warm } \\
\mathrm{Cu}\end{array}$ & $\begin{array}{l}\text { Recirc. } \\
\text { Warm } \\
\mathrm{Cu}\end{array}$ & $\begin{array}{c}\text { Recirc. } \\
\text { Warm } \\
\mathrm{Cu}\end{array}$ & Sums \\
\hline $\begin{array}{l}E^{\text {init }}(\mathrm{GeV}) \\
E^{\text {final }}(\mathrm{GeV})\end{array}$ & $\begin{array}{l}0.10 \\
0.20\end{array}$ & $\begin{array}{l}0.20 \\
0.70\end{array}$ & $\begin{array}{l}0.70 \\
2\end{array}$ & $\begin{array}{l}2 \\
7\end{array}$ & $\begin{array}{r}7 \\
50\end{array}$ & \\
\hline $\begin{array}{l}\text { Circ. }(\mathrm{km}) \\
\text { Turns } \\
\text { Loss }(\%) \\
\text { Decay heat }(\mathrm{W} / \mathrm{m}) \\
B_{\text {fixed }}(\mathrm{T}) \\
\text { Ramp freq. }(\mathrm{kHz})\end{array}$ & $\begin{array}{l}0.04 \\
1 \\
2.31 \\
0.89\end{array}$ & $\begin{array}{l}0.07 \\
1 \\
3.98 \\
1.98\end{array}$ & $\begin{array}{l}0.07 \\
8 \\
7.27 \\
11.04 \\
2 \\
281\end{array}$ & $\begin{array}{c}0.19 \\
10 \\
7.91 \\
12.99 \\
2 \\
79.83\end{array}$ & $\begin{array}{c}1.74 \\
11 \\
13.94 \\
12.44 \\
2 \\
8.00\end{array}$ & 31.06 \\
\hline Disp (m) & & & 1 & 1.50 & 3 & \\
\hline $\begin{array}{l}\beta_{\max }(\mathrm{m}) \\
\sigma_{\text {init }}(\mathrm{cm})\end{array}$ & $\begin{array}{l}0.83 \\
2.71\end{array}$ & $\begin{array}{l}1.42 \\
2.22\end{array}$ & $\begin{array}{l}3.00 \\
1.42\end{array}$ & $\begin{array}{l}5.31 \\
1.64\end{array}$ & $\begin{array}{r}21.08 \\
0.90\end{array}$ & \\
\hline$\Delta p / p^{\text {init }}(\%)$ & 3.58 & 2.80 & 1.64 & 0.56 & 0.32 & \\
\hline$\sigma_{y}(\mathrm{~cm})$ & 1.09 & 1.14 & 1.01 & 0.85 & 0.94 & \\
\hline$\sigma_{x}(\mathrm{~cm})$ & & & 1.93 & 1.19 & 1.34 & \\
\hline Pipe full height $(\mathrm{cm})$ & 10.92 & 11.42 & 10.14 & 8.49 & 9.39 & \\
\hline Pipe full width $(\mathrm{cm})$ & 10.92 & 11.42 & 19.28 & 11.94 & 13.35 & \\
\hline rf freq. (MHz) & 200 & 200 & 200 & 200 & 400 & \\
\hline Acc./turn $(\mathrm{GeV})$ & 0.20 & 0.40 & 0.17 & 0.50 & 4 & \\
\hline Acc. time $(\mu \mathrm{s})$ & & & 1 & 6 & 62 & \\
\hline$\eta(\%)$ & 5.15 & 5.36 & 6.36 & 2.84 & 6.92 & \\
\hline Acc. grad. (MV/m) & 8 & 8 & 8 & 10 & 10 & \\
\hline Synch. rot's & 0.62 & 0.63 & 0.62 & 3.92 & 23.16 & \\
\hline Cavity rad. $(\mathrm{cm})$ & 54.37 & 54.88 & 54.88 & 60.47 & 38.26 & \\
\hline Beam time (ms) & 0.00 & 0.00 & 0.00 & 0.01 & 0.06 & \\
\hline rf time (ms) & 0.17 & 0.17 & 0.17 & 0.18 & 0.13 & \\
\hline Tot. peak rf (GW) & 0.05 & 0.10 & 0.05 & 0.26 & 4.71 & 5.17 \\
\hline Ave. rf power (MW) & 0.14 & 0.24 & 0.13 & 0.68 & 9.54 & 10.73 \\
\hline rf wall (MW) & 0.64 & 1.16 & 0.46 & 2.42 & 28.06 & 32.75 \\
\hline
\end{tabular}


spread in the first recirculating accelerator would be nearly $6 \% \mathrm{rms}$, or about $50 \%$ full width. This is a very large momentum spread that could be accepted only in FFAG-like lattices as discussed below.

Similarly, if the same acceleration is to be usable as the front end for a $250+250 \mathrm{GeV}$ or higher energy machine, then the transverse emittance will again be less, the longitudinal emittance even larger, and the problem of very large momentum spread will be worse. Clearly, although not absolutely needed for a Higgs factory, it is desirable to solve this problem even in the first collider.

A separate parameter set for the high luminosity $50+$ $50 \mathrm{GeV}$ collider and a $250+250 \mathrm{GeV}$ collider could have been presented, but their parameters are very similar to those of the front end of the $3 \mathrm{TeV}$ machine given below, and are thus omitted here.

\section{Acceleration for $3 \mathrm{TeV}$ collider}

For a high energy machine, the muon accelerators are physically the largest component and are also probably the most expensive. More work is needed on its design. Table $\mathrm{X}$ gives an early example of a possible sequence of accelerators for a $3 \mathrm{TeV}$ collider.

Linacs are used up to $700 \mathrm{MeV}$, followed by recirculating linac accelerators. In the first of these, because of the very large longitudinal emittance, the momentum spread as the beam enters the first recirculating linac accelerator is $8.5 \% \mathrm{rms}$, which is very large. The lattice must have very strong focusing, small dispersion, and large aperture. If this is not possible, higher energy linacs or lower frequency rf could relieve the requirement.

For the final three stages, pulsed magnet synchrotrons [178] are used. In the $200 \mathrm{GeV}$ ring, all the magnets in the ring are pulsed, but in the last two rings a superconducting pulsed hybrid solution is used. In these cases, if only pulsed magnets were used, then the power consumed would be too high, and, because only low pulsed fields could be used, the circumferences would also be very large. It is thus proposed to use rings with alternating warm pulsed magnets and superconducting fixed magnets [179] (see Fig. 38). The fixed magnets are

TABLE X. Parameters of acceleration for a $3 \mathrm{TeV}$ collider.

\begin{tabular}{|c|c|c|c|c|c|c|c|c|}
\hline $\begin{array}{l}\text { Acc. type } \\
\text { Magnet type } \\
\text { rf type }\end{array}$ & $\begin{array}{l}\text { Linac } \\
\mathrm{Cu}\end{array}$ & $\begin{array}{l}\text { Recirc. } \\
\text { Warm } \\
\mathrm{Cu}\end{array}$ & $\begin{array}{l}\text { Recirc. } \\
\text { Warm } \\
\mathrm{Cu}\end{array}$ & $\begin{array}{l}\text { Recirc. } \\
\text { Warm } \\
\text { SC Nb }\end{array}$ & $\begin{array}{l}\text { Synch. } \\
\text { Warm } \\
\text { SC Nb }\end{array}$ & $\begin{array}{l}\text { Synch. } \\
\text { Hybrid } \\
\text { SC Nb }\end{array}$ & $\begin{array}{l}\text { Synch. } \\
\text { Hybrid } \\
\text { SC Nb }\end{array}$ & Sums \\
\hline $\begin{array}{l}E^{\text {init }}(\mathrm{GeV}) \\
E^{\text {final }}(\mathrm{GeV})\end{array}$ & $\begin{array}{l}0.10 \\
0.70\end{array}$ & $\begin{array}{l}0.70 \\
2\end{array}$ & $\begin{array}{l}2 \\
7\end{array}$ & $\begin{array}{r}7 \\
50\end{array}$ & $\begin{array}{r}50 \\
200\end{array}$ & $\begin{array}{r}200 \\
1000\end{array}$ & $\begin{array}{l}1000 \\
1500\end{array}$ & \\
\hline Circ. $(\mathrm{km})$ & 0.07 & 0.12 & 0.26 & 1.74 & 4.65 & 11.30 & 11.36 & \\
\hline Turns & 2 & 8 & 10 & 11 & 15 & 27 & 17 & \\
\hline Loss $(\%)$ & 6.11 & 12.28 & 10.84 & 13.94 & 10.68 & 10.07 & 2.65 & 50.58 \\
\hline Decay heat $(\mathrm{W} / \mathrm{m})$ & 3.67 & 15.02 & 16.89 & 15.91 & 19.44 & 30.97 & 18.09 & \\
\hline $\begin{array}{l}B_{\text {pulse }}(\mathrm{T}) \\
B_{\text {fixed }}(\mathrm{T}) \\
\text { Frac pulsed }(\%) \\
\text { Ramp freq. }(\mathrm{kHz})\end{array}$ & & 0.70 & 57.34 & 8.00 & 2.15 & $\begin{array}{c}2 \\
8 \\
73 \\
0.50\end{array}$ & $\begin{array}{c}2 \\
8 \\
43 \\
0.79\end{array}$ & \\
\hline Disp. (m) & & 0.40 & 0.60 & 0.80 & 1 & 2 & 4 & \\
\hline$\beta_{\max }(\mathrm{m})$ & 0.89 & 3.97 & $\begin{array}{r}8.75 \\
-0.25\end{array}$ & $\begin{array}{r}36.29 \\
-0.50\end{array}$ & $\begin{array}{r}52.20 \\
-0.50\end{array}$ & $\begin{array}{l}108 \\
-0,50\end{array}$ & $\begin{array}{r}120 \\
-1\end{array}$ & \\
\hline$\sigma_{z}^{\text {init }}(\mathrm{cm})$ & 16.34 & 8.53 & 5.29 & 3.57 & 1.59 & 0.96 & 0.78 & \\
\hline$\Delta^{z} p / p^{\text {init }}(\%)$ & 19.27 & 8.49 & 5.41 & 2.47 & 0.82 & 0.35 & 0.09 & \\
\hline$\sigma_{y}(\mathrm{~cm})$ & 0.45 & 0.45 & 0.42 & 0.48 & 0.22 & 0.16 & 0.08 & \\
\hline$\sigma_{x}(\mathrm{~cm})$ & & 3.40 & 3.25 & 1.98 & 0.82 & 0.71 & 0.36 & \\
\hline Pipe full height $(\mathrm{cm})$ & 4.46 & 4.52 & 4.22 & 4.77 & 2.20 & 1.62 & 0.78 & \\
\hline Pipe full width $(\mathrm{cm})$ & 4.46 & 33.95 & 32.49 & 19.79 & 8.20 & 7.06 & 3.62 & \\
\hline rf freq $(\mathrm{MHz})$ & 200 & 100 & 200 & 200 & 800 & 1300 & 1300 & \\
\hline Acc./turn $(\mathrm{GeV})$ & 0.40 & 0.17 & 0.50 & 4 & 10 & 30 & 30 & \\
\hline Acc. time $(\mu \mathrm{s})$ & & 3 & 8 & 62 & 232 & 1004 & 631 & \\
\hline$\eta(\%)$ & 3.82 & 0.96 & 1.97 & 1.11 & 10.15 & 14.37 & 12.92 & \\
\hline Acc. grad. (MV/m) & 8 & 8 & 10 & 10 & 15 & 25 & 25 & \\
\hline Synch. rot's & 0.81 & 0.76 & 1.02 & 5.82 & 19.14 & 54.29 & 31.30 & \\
\hline Cavity rad. (cm) & 54.88 & 110 & 60.47 & 76.52 & 19.13 & 11.77 & 11.77 & \\
\hline rf time (ms) & 0.04 & 0.12 & 0.05 & 0.56 & 0.40 & 1.25 & 0.96 & \\
\hline Tot. peak rf (GW) & 0.21 & 0.14 & 0.59 & 1.31 & 1.06 & 1.16 & 1.04 & 5.51 \\
\hline Ave. rf power (MW) & 0.14 & 0.25 & 0.45 & 11.04 & 6.32 & 21.91 & 15.07 & 55.18 \\
\hline rf wall (MW) & 0.64 & 0.88 & 1.62 & 32.47 & 18.59 & 44.72 & 30.76 & 130 \\
\hline
\end{tabular}



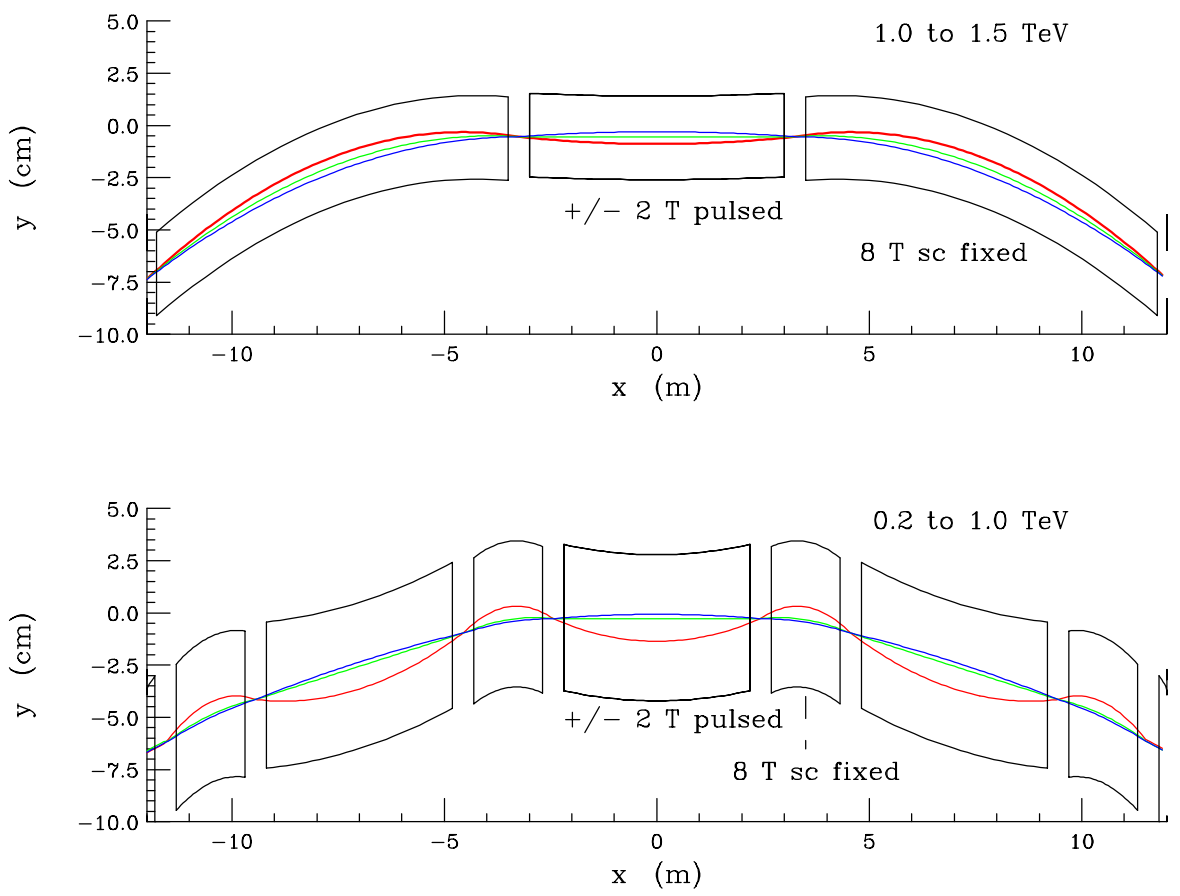

FIG. 38. (Color) Schematic of hybrid superconducting-pulse magnet accelerator ring.

superconducting at $8 \mathrm{~T}$; the pulsed magnets are warm with fields that swing from -2 to $+2 \mathrm{~T}$. The effective ramp frequency is given in the table. Both of these rings are in the same tunnel, with the fraction of magnet length pulsed (vs fixed) being different ( $73 \%$ and $43 \%$ ).

In all the final three rings, superconducting $\mathrm{rf}$ is employed to minimize the peak power requirements and to obtain high wall-to-beam efficiency and thus keep the wall power consumption reasonable. In the final two rings the frequency and cavity designs are chosen to be the same as that in the TESLA [180] proposal.

\section{Design issues}

\section{Recirculating linac accelerator lattice issues}

Beam transport R\&D for recirculating linac accelerators follows the model of the TJNAF. The layout is a racetrack with linacs in the straight sections and multipass return arcs. At the ends of the linacs the multipass beam lines are recombined. A pulse magnet at each separation/recombination point is used to guide the beam into the energy matched return arc. Some initial lattice design concepts for recirculating linac accelerators are being developed. The basic return arc unit would be a FODO lattice, but with the quadrupole strengths varied in order to perturb the arc dispersion function and obtain nearly isochronous motion around the arcs. The arcs are dispersion matched by setting the arc phase advance to a multiple of $2 \pi$. Arc designs based upon the flexible momentum compaction module can also be used.
In the special case of the very low momentum spread Higgs factory, the transverse emittances are very large ( $\approx 300 \pi \mathrm{mm} \mathrm{mrad}, \mathrm{rms}$ ), and will require strong focusing in the lattices. Momentum acceptance in the rings is, in this case, not a problem. But the longitudinal phase space of the muons in the other machines is much larger and requires, at low energies, either long bunches or large momentum spreads. The requirement of high accelerating gradients argues for high frequencies, and thus short bunches. One therefore needs accelerators with large momentum acceptances.

In the $3 \mathrm{TeV}$ example above, the acceptance at injection into the first recirculating accelerator is $8.5 \% \mathrm{rms}$. This is very large by conventional standards, but far less than that in the FFAG lattices being studied [174]. Thus the early return arcs of such a recirculating linac accelerator would have to have very strong focusing, and be FFAG-like. Of course, if a true FFAG accelerator were to be used for its avoidance of the switchyards and multiaperture magnets, then the specified momentum spread would certainly not be a problem.

Permanent, ferric or superferric $(\approx 2 \mathrm{~T})$, or high field superconducting magnets could be used for recirculating linac accelerators. The lower field magnets may be economic for initial turns, while high field magnets minimize particle travel times, and therefore decay losses. Designs for multiaperture superconducting magnets suitable for recirculating linac accelerators have been developed [44], and superconducting magnets with as many as 18 apertures with $0.7-7 \mathrm{~T}$ fields have been designed. A variety of magnet configurations can be developed; 
cost/performance optimization will be needed in developing a final choice.

\section{2. rf peak power requirements}

Because of the need for rapid acceleration, the peak rf powers are high, and the resulting numbers of power sources large. For the linacs and early recirculating accelerators, the powers are high because of the high gradients and low frequencies needed to accelerate the long bunches. At these frequencies $(\approx 200 \mathrm{MHz})$, currently available sources (triodes and tetrodes) have relatively low maximum output powers and are expensive. Low temperature operation of the cavities, and superconducting or conventional rf compressor power [181] systems, which would reduce the peak power requirements, are being considered.

Study of the example suggests that in the first two recirculators (up to $7 \mathrm{GeV}$ ) there is no hope for the rf to keep up with the beam loading. The cavity can be filled only in a suitable filling time (twice the time constant in these examples), and the rf voltage allowed to sag as the beam makes its multiple passes. If excessive sensitivity to beam current is to be avoided, then the stored energy must be large compared to that used, which is somewhat inefficient.

In the final recirculating accelerator, continuous filling (cw) is just possible, but requires yet higher peak power $(\approx 5 \mathrm{GW}$ total at $400 \mathrm{MHz}$ ) because of the high acceleration rate. The use of superconducting cavities can reduce losses, and thus reduce this peak rf power source requirement, and was included in the above $3 \mathrm{TeV}$ example. At this frequency $(400 \mathrm{MHz})$, klystrons are available with greater power $(\approx 20 \mathrm{MW})$ than that of the sources at the lower frequencies, but a yet higher power klystron (50-100 MW) could probably be developed and would be desirable.

\section{Pulsed magnet systems}

A pulsed current $4 \mathrm{~T}$ magnet has been designed for acceleration to $250 \mathrm{GeV}$ in $360 \mu$ s [182], but efficiency favors use of ferric materials in rapid acceleration magnets, although this would limit peak magnetic fields to $\approx 2 \mathrm{~T}$. The average field can be increased by interleaving magnets swinging from -2 to $2 \mathrm{~T}$ with fixed field $8 \mathrm{~T}$ superconducting magnets.

Faster pulsing magnets would require special materials to minimize energy losses from eddy currents. Options include silicon steel, metglass laminations, or finemet laminated tape or powdered solid. A $30 \mu \mathrm{m}$ metglass lamination suitable for several $\mathrm{kHz}$ cycling has been developed. A design of suitable pulsed magnets [179] (see Fig. 39) has been shown to have sufficiently low losses for this application. The magnets employ cables made of many fine insulated strands (litz cable) and the yokes are made of very thin $(0.28 \mathrm{~mm}), 3 \% \mathrm{Si}$-Fe laminations, possibly of metglas $[183,184]$ for the higher rate cases. Detailed designs must be developed, prototypes constructed, and the practical limits of recycling scenarios should be determined.

\section{Superconducting linacs}

While the gradients needed in the acceleration systems are not excessive, they are larger than previous experience at the lower frequencies. The high peak power pulsed operation poses power handling difficulties at lower energies and high peak current presents collective effect (wake field) difficulties at higher energies. Higher gradients and efficiencies in all sections would improve performance.

The superconducting rf would operate in pulsed mode, matched to the acceleration time of up to a few ms.

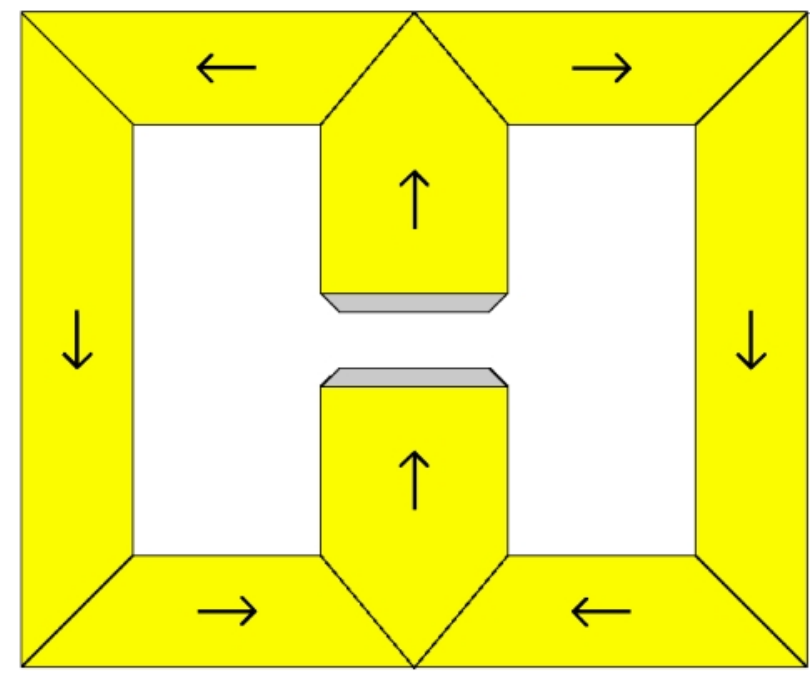

FIG. 39. (Color) A 2D picture of an $H$ frame magnet lamination with grain oriented 3\% Si-Fe steel. The arrows show both the magnetic field and the grain direction. 
This pulse structure is similar to the multibunch acceleration mode planned for TESLA $(25 \mathrm{MV} / \mathrm{m}$ at $1300 \mathrm{MHz}$ designs), and studies indicate that this design could be adapted to $\mu^{+}-\mu^{-}$acceleration. At lower frequencies, structures such as the CERN $350 \mathrm{MHz}$ superconducting rf cavities could be used. These cavities have been tested in pulsed mode operation, and tests indicate that pulsed acceleration fields $>10 \mathrm{MV} / \mathrm{m}$ are possible [185].

The high single bunch intensities required for high intensities imply large higher order mode (HOM) losses and large wake field effects from the short, high intensity bunches. Higher order mode load designs adapted from superconducting rf experience could be used. HOM loads and wake fields are expected to vary as $a^{-2}, \lambda^{-2}$, and $\sigma^{-1 / 2}$, where $a$ is the cavity aperture, $\lambda$ is the acceleration wavelength, and $\sigma$ is the bunch length [186,187]. Calculations indicate that the wake fields would limit bunch intensities to $\approx 2 \times 10^{12}$ with $1300 \mathrm{MHz}$ superconducting rf in a recirculating linac accelerator scenario. The longitudinal dynamics is microtronlike or synchrotronlike and off-crest acceleration enables compensation of the linear part of the wake fields, with synchrotronlike phase stability [176].

\section{E. Simulations}

A study [177] followed the longitudinal motion of particles through a similar sequence of recirculating accelerators (see Table XI). Cavities similar to those proposed for TESLA [180] were assumed. Figure 40 shows, after optimization of parameters, the final longitudinal phase space distributions corresponding to wake fields estimated for four different bunch charges: (a) very small, (b) $0.83 \times 10^{12}$ muons, (c) $2.08 \times 10^{12}$ muons, and (d) $4.17 \times 10^{12}$ muons.

For the design beam charge of $2 \times 10^{12}$ muons [approximately as for Fig. 40(c)], the wake field amplitude was estimated to be $2.5 \mathrm{MV} / \mathrm{m}$, the accelerating phase was $35^{\circ}$, and rf voltage depression $26 \%$. The simulation used an initial longitudinal phase space of $20 \mathrm{eV}$ s. It gave negligible particle loss, a final longitudinal phase space of $21.6 \mathrm{eV} \mathrm{s}$, resulting in an increase of longitudinal emittance of only $8 \%$.

\section{F. Acceleration research needed}

As discussed above, possible acceleration configurations have been developed and critical longitudinal motion simulations have been performed. These calculations support the general feasibility of acceleration of muons from cooling to collider energies. However, the designs of acceleration systems have not been fully detailed and much work would be needed to obtain a buildable design. Complete transport lattices for linacs and return arcs have not yet been derived, and 6D phase space tracking of beams through the accelerators has not been attempted. Also, the geometry of combining and separating multipass beams has not been worked out and optimized.

The rf requirements and systems have been specified at only the rudimentary requirements level, and have not been developed to a constructible level. Optimal configurations and choices of normal or superconducting rf must be developed, as well as more optimal choices in acceleration frequencies. The simple wake field models used in the initial simulations should be expanded to obtain more realistic systems, and more precise calculations of wake field effects must be developed.

Rapid accelerating systems have been outlined only at the simplest conceptual level. Prototype magnet design and testing are needed to test the limits of cycling rate and field strengths. Successful magnet concepts must then be specified in terms of stable beam transport configurations, including focusing and transport matching. While beam is stored for only a few turns, the individual bunch intensities are large enough that the possibility of single bunch instabilities must be considered and calculated. The larger number of passes in a recirculating linac accelerator places greater demands on the rf systems and higher order mode loads, particularly for superconducting systems.

TABLE XI. Parameters of acceleration for a $4 \mathrm{TeV}$ collider.

\begin{tabular}{|c|c|c|c|c|c|}
\hline & Linac & RLA1 & RLA2 & RCS1 & $\mathrm{RCS} 2$ \\
\hline$E(\mathrm{GeV})$ & $0.1 \rightarrow 1.5$ & $1.5 \rightarrow 10$ & $10 \rightarrow 70$ & $70 \rightarrow 250$ & $250 \rightarrow 2000$ \\
\hline$f_{\mathrm{rf}}(\mathrm{MHz})$ & $30 \rightarrow 100$ & 200 & 400 & 800 & 1300 \\
\hline$N_{\text {turns }}$ & 1 & 9 & 11 & 33 & 45 \\
\hline$V_{\mathrm{rf}}(\mathrm{GV} /$ turn $)$ & 1.5 & 1.0 & 6 & 6.5 & 42 \\
\hline$C_{\text {turn }}(\mathrm{km})$ & 0.3 & 0.16 & 1.1 & 2.0 & 11.5 \\
\hline Beam time $(\mathrm{ms})$ & 0.0013 & 0.005 & 0.04 & 0.22 & 1.73 \\
\hline$\sigma_{z, \text { beam }}(\mathrm{cm})$ & $50 \rightarrow 8$ & $4 \rightarrow 1.7$ & $1.7 \rightarrow 0.5$ & $0.5 \rightarrow 0.25$ & $0.25 \rightarrow 0.12$ \\
\hline$\sigma_{E, \text { beam }}(\mathrm{GeV})$ & $0.05 \rightarrow 0.033$ & $0.067 \rightarrow 0.16$ & $0.16 \rightarrow 0.58$ & $0.58 \rightarrow 1.14$ & $1.14 \rightarrow 2.3$ \\
\hline Loss $(\%)$ & 5 & 7 & 6 & 7 & 10 \\
\hline
\end{tabular}



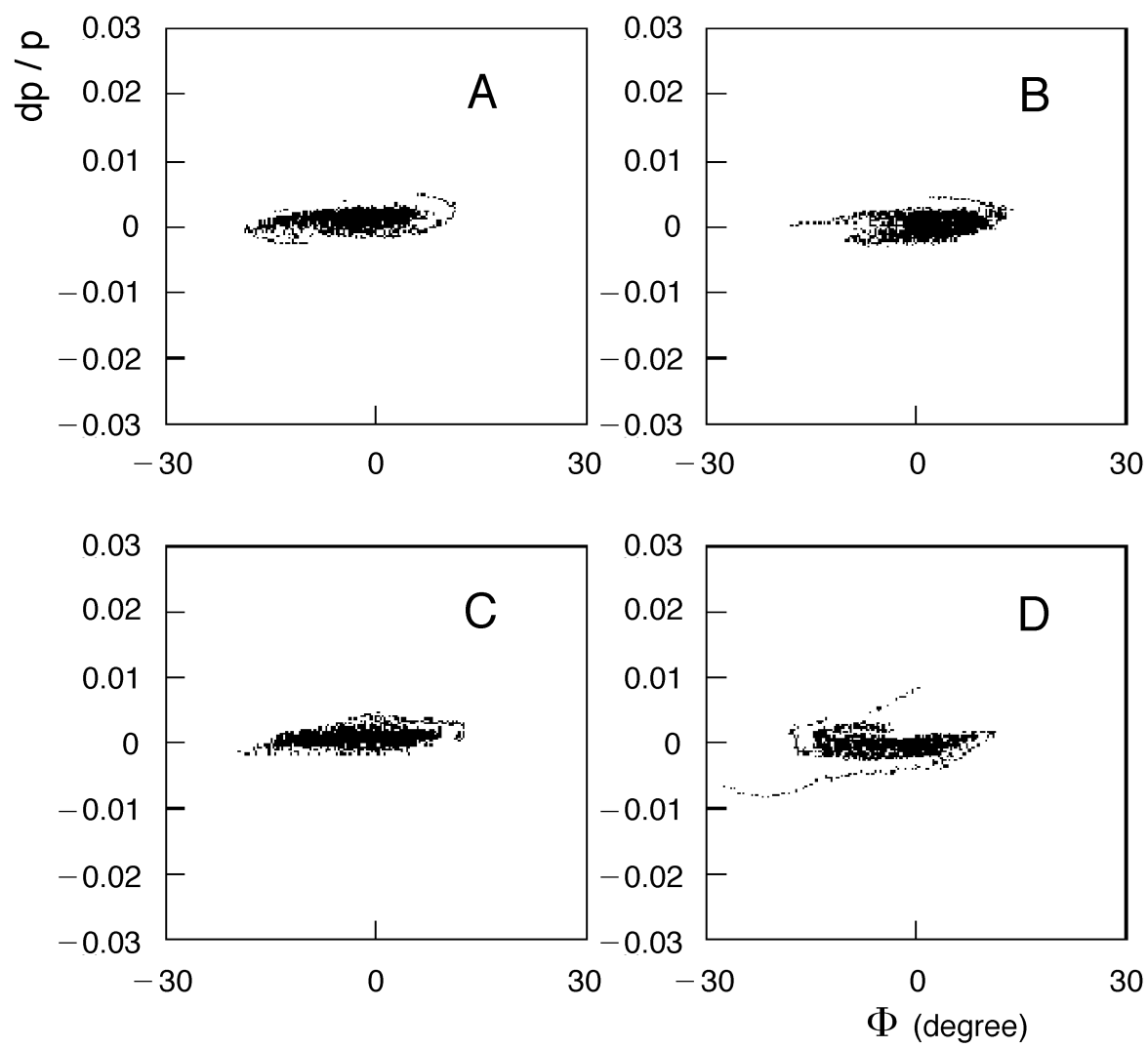

FIG. 40. Recirculating linac accelerator simulation results with wake fields, with beam accelerated from 200 to $2000 \mathrm{GeV}$ in a ten-turn recirculating linac accelerator. Longitudinal phase space plots for different bunch charges: (A) very small number, (B) $0.83 \times 10^{12}$, (C) $2.08 \times 10^{12}$, and (D) $4.17 \times 10^{12}$ muons in a bunch.

\section{COLLIDER STORAGE RING A. Introduction}

After one $\mu^{+}$bunch and one $\mu^{-}$bunch have been accelerated to collision energy, the two bunches are injected into the collider ring, which is a fixed field storage ring. Parameters for several possible collider storage rings are given in Table I. Collider ring lattices have been developed for two of the collision energies in this table: $100 \mathrm{GeV}$ and $3 \mathrm{TeV}$ in the center of mass.

Three operational modes are proposed in the above table for the $100 \mathrm{GeV}$ collider, each requiring different machine optics. The following sections discuss a $100 \mathrm{GeV}$ collider lattice for two of the modes, the broad momentum spread case $(\Delta p / p$ of $0.12 \%, \mathrm{rms})$ and the narrow momentum spread case $(\Delta p / p$ of $0.003 \%)$, as well as a $3 \mathrm{TeV}$ collider lattice.

\section{B. Collider lattices 1. Design criteria}

Stringent criteria have been imposed on the collider lattice designs in order to attain the specified luminosities. The first and most difficult criterion to satisfy is the provision of an interaction region (IR) with extremely low $\beta^{*}$ values at the collision point consistent with ac- ceptable dynamic aperture. The required $\beta^{*}$ values for the $100 \mathrm{GeV}$ collider are $4 \mathrm{~cm}$ for the broad momentum spread case and $14 \mathrm{~cm}$ for the narrow momentum spread case. For the $3 \mathrm{TeV}$ machine, $\beta^{*}$ is only $3 \mathrm{~mm}$. These $\beta^{*}$ values were tailored to match the longitudinal bunch lengths in order to avoid luminosity dilution from the hourglass effect. Achieving this requirement in the $3 \mathrm{TeV}$ lattice is complicated by the high peak beta function values in the final focus quadrupoles requiring 8$10 \mathrm{~cm}$ radial apertures. The correspondingly weakened gradients combined with the ultrahigh energy make for a long final focus structure. (In contrast, the lower energy and larger $\beta^{*}$ values in the $100 \mathrm{GeV}$ collider lead to an efficient, compact final focus telescope.) Compounding the problem, particularly for the $3 \mathrm{TeV}$ design, is the need to protect the superconducting coils from the decay products of the muons. Placing a tungsten shield between the vacuum chamber and the coils can increase the radial aperture in the $3 \mathrm{TeV}$ quadrupoles by as much as $6 \mathrm{~cm}$, lowering available gradients still further. Final focus designs must also include collimators and background sweep dipoles, and other provisions for protecting the magnets and detectors from muon decay electrons. Effective schemes have been incorporated into the current lattices. 
Another difficult constraint imposed on the lattice is that of isochronicity. A high degree of isochronicity is required in order to maintain the short bunch structure without excessive rf voltage. In the lattices presented here, control over the momentum compaction is achieved through appropriate design of the arcs.

A final criterion especially important in the lower energy colliders is that the ring circumference be as small as feasible in order to minimize luminosity degradation through decay of the muons. Achieving small circumference requires high fields in the bending magnets as well as a compact, high dipole packing fraction design. To meet the small circumference demand, $8 \mathrm{~T}$ pole tip fields have been assumed for all superconducting magnets, with the exception of the $3 \mathrm{TeV}$ final focus quadrupoles, whose pole tips are assumed to be as high as $12 \mathrm{~T}$. In addition, design studies for still higher field dipoles are in progress.

\section{2. rf system}

The rf requirements depend on the momentum compaction of the lattice and on the parameters of the muon bunch. For the case of very low momentum spread, synchrotron motion is negligible and the rf system is used solely to correct an energy spread generated through the impedance of the machine. For the cases of higher momentum spreads, there are two approaches. One is to make the momentum compaction zero to high order through lattice design. Then the synchrotron motion can be eliminated, and the rf is again only needed to compensate the induced energy spread correction. Alternatively, if some momentum compaction is retained, then a more powerful $\mathrm{rf}$ system is needed to maintain the specified short bunches. In either case, rf quadrupoles will be required to generate BNS (after Balakin, Novokhatsky, and Smirnov) [188,189] damping of the transverse head-tail instability.

\section{3. $3 \mathrm{TeV}$ COM lattice}

The $3 \mathrm{TeV}$ ring has a roughly racetrack design with two circular arcs separated by an experimental insertion on one side, and a utility insertion for injection, extraction, and beam scraping on the other. The experimental insertion includes the interaction region followed by a local chromatic correction section (CCS) and a matching section. The chromatic correction section is optimized to correct the ring's linear chromaticity, which is mostly generated by the low beta quadrupoles in the IR. In designs of $e^{+} e^{-}$colliders, it has been found that local chromatic correction of the final focus is essential [190193], as was found to be the case here. The $3 \mathrm{TeV}$ IR and $\mathrm{CCS}$ are displayed in Fig. 41. The accompanying $3 \mathrm{TeV}$ arc module in Fig. 41 is an example of a module which controls momentum compaction (i.e., isochronicity) of the entire ring.
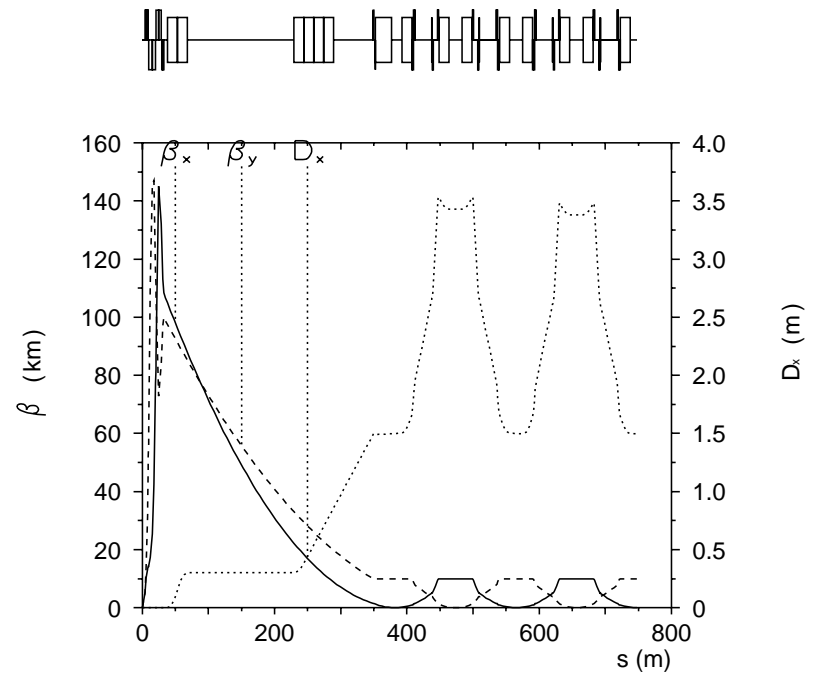

FIG. 41. Example (a): $3 \mathrm{TeV}$ IR and chromatic correction section.

\section{4. $100 \mathrm{GeV}$ COM lattices}

For the $100 \mathrm{GeV} \mathrm{COM}$ collider [194], two operating modes are contemplated: a high luminosity case with broad momentum acceptance to accommodate a beam with a $\Delta p / p$ of $\pm 0.12 \%$ (rms), and one with a much narrower momentum acceptance and lower luminosity for a beam with $\Delta p / p$ of $\pm 0.003 \%$ (rms). For the broad momentum acceptance case, $\beta^{*}$ must be $4 \mathrm{~cm}$ and for the narrow momentum acceptance case, $14 \mathrm{~cm}$. In either case, the bunch length must be held comparable to the value of $\beta^{*}$. The $100 \mathrm{GeV}$ ring geometry is highly compact and more complicated than a racetrack, but the lattice has regions with the same functions as those of the $3 \mathrm{TeV}$ ring.

Two independent $100 \mathrm{GeV}$ lattice designs have evolved; these are described below in separate sections and denoted Example (a) and Example (b), respectively.
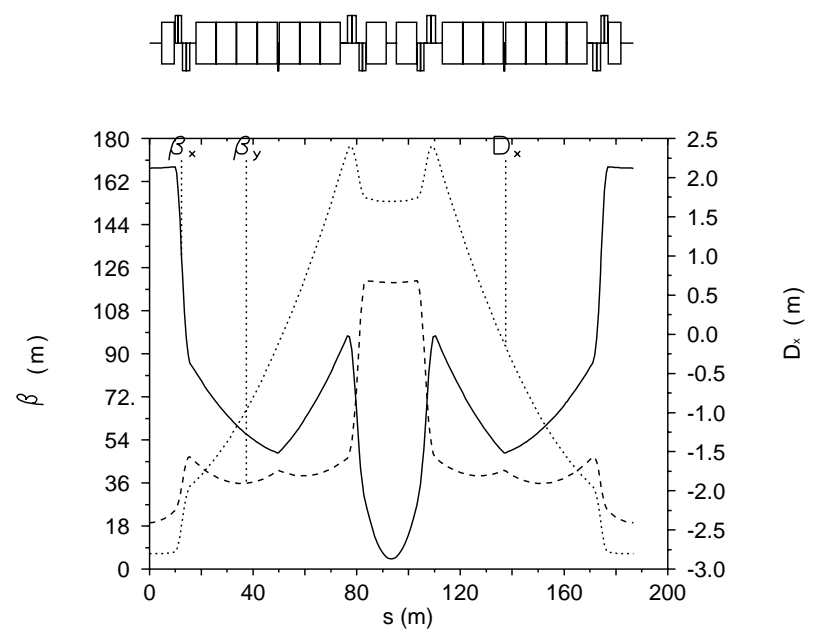

FIG. 42. Example (a): $3 \mathrm{TeV}$ arc module. 
The first design described is a lattice which has two optics modes. In the high luminosity mode, the $\beta^{*}$ value is $4 \mathrm{~cm}$ with a transverse and momentum aperture sufficient to accept a normalized beam emittance of $90 \pi$ (rms) and a $\Delta p / p$ of $\pm 0.12 \%$ (rms). The second, lower luminosity mode has a $\beta^{*}$ value of $14 \mathrm{~cm}$ with a very large transverse acceptance, but small, approximately monochromatic, momentum acceptance.

The second $100 \mathrm{GeV}$ lattice described is another collider design with a $4 \mathrm{~cm} \beta^{*}$ optics mode. Although the number of magnets differ between the two lattices, the most important optics difference between the two is in the modules used in the arcs.

\section{5. $100 \mathrm{GeV} \mathrm{COM-Example} \mathrm{(a)}$}

The need for different collision modes in the $100 \mathrm{GeV}$ machine led to an interaction region design with two optics modes: one with broad momentum acceptance $(\Delta p / p$ of $0.12 \%$, rms $)$ and a collision $\beta^{*}$ of $4 \mathrm{~cm}$, and the other basically monochromatic $(\Delta p / p$ of $0.003 \%$, rms) and a larger collision $\beta^{*}$ of $14 \mathrm{~cm}$. The first lattice design, denoted Example (a), shown in Figs. 53 and 54, has a total circumference of about $350 \mathrm{~m}$ with arc modules accounting for only about one-quarter of the ring circumference.

The low beta function values at the interaction point (IP) are mainly produced by three strong superconducting quadrupoles in the Final Focus Telescope (FFT) with pole tip fields of $8 \mathrm{~T}$. The full interaction region is symmetric under reflection about the IP. Because of significant, large angle backgrounds from muon decay, a background sweep dipole is included in the final focus telescope and placed near the IP to protect the detector and the low $\beta$ quadrupoles [195]. It was found that this sweep dipole, $2.5 \mathrm{~m}$ long with an $8 \mathrm{~T}$ field, provides sufficient background suppression. The first quadrupole is



FIG. 43. Example (a): $4 \mathrm{~cm} \beta^{*}$ mode showing half of the IR, local chromatic correction section, and one of three arc modules. located $5 \mathrm{~m}$ away from the interaction point, and the beta functions reach a maximum value of $1.5 \mathrm{~km}$ in the final focus telescope, when the maxima of the beta functions in both planes are equalized. For this maximum beta value, the quadrupole apertures must be at least $11 \mathrm{~cm}$ in radius to accommodate $5 \sigma$ of a $90 \pi \mathrm{mm} \mathrm{mrad}, 50 \mathrm{GeV}$ muon beam (normalized rms emittance) plus a $2-3 \mathrm{~cm}$ thick tungsten liner [196]. The natural chromaticity of this interaction region is about -60 .

Local chromatic correction of the muon collider interaction region is required to achieve broad momentum acceptance. The basic approach developed by Brown [191] and Donald et al. [197] is implemented in the chromatic correction region (CC). The CC contains two pairs of sextupoles, one pair for each transverse plane, all located at locations with high dispersion. The sextupoles of each pair are located at positions of equal, high beta value in the plane (horizontal or vertical) whose chromaticity is to be corrected, and very low beta waist in the other plane. Moreover, the two sextupoles of each pair are separated by a betatron phase advance of near $\pi$, and each sextupole has a phase separation of $(2 n+1) \frac{\pi}{2}$ from the IP, where $n$ is an integer. The result of this arrangement is that the geometric aberrations of each sextupole are canceled by its companion while the chromaticity corrections add.

The sextupoles of each pair are centered about a minimum in the opposite plane $\left(\beta_{\min }<1 \mathrm{~m}\right)$, which provides chromatic correction with minimal cross correlation between the planes. A further advantage to locating the opposite plane's minimum at the center of the sextupole, is that this point is $\frac{\pi}{2}$ away from, or "out of phase" with, the source of chromatic effects in the final focus quadrupoles; i.e., the plane not being chromatically corrected is treated like the IP in terms of phase to eliminate a second-order chromatic aberration generated by an "opposite-plane" sextupole.

In this lattice example, the CC (Fig. 45) was optimized to be as short as possible. The $\beta_{\max }$ is only $100 \mathrm{~m}$ and


FIG. 44. Example (a): $14 \mathrm{~cm} \beta^{*}$ mode showing half of the IR, local chromatic correction, and one of three arc modules. 

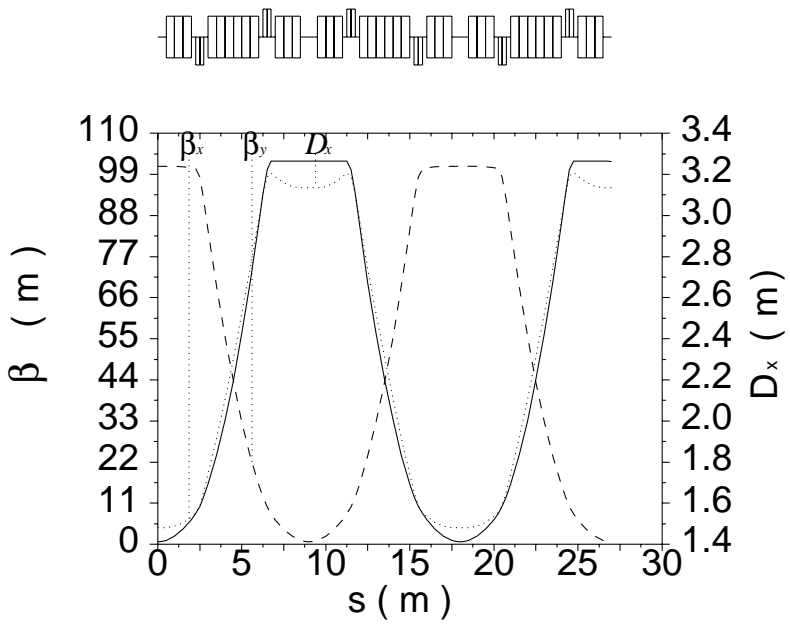

FIG. 45. Example (a): The chromatic correction module.

the $\beta_{\min }=0.7 \mathrm{~m}$, giving a $\beta_{\text {ratio }}$ between planes of about 150 , so the dynamic aperture is not compromised by a large amplitude dependent tune shift.

This large beta ratio, combined with the opposite plane phasing, allows the sextupoles for the opposite planes to be interleaved, without significantly increasing the nonlinearity of the lattice. In fact, interleaving improved lattice performance compared to that of a noninterleaved correction scheme, due to a shortening of the chromatic correction section, which lowers its chromaticity contribution [198]. The use of somewhat shallower beta minima with less variation in beta through the sextupoles was made to soften the chromatic aberrations, although this caused a slight violation of the exact $\pi$ phase advance separation between sextupole partners. The retention of an exact $\pi$ phase advance difference between sextupoles was found to be less important to the dynamic aperture than elimination of minima with $\beta_{\min }<0.5 \mathrm{~m}$.

The total momentum compaction contributions of the IR, CC, and matching sections is about 0.04. The total length of these parts is $173 \mathrm{~m}$, while that of the momentum compaction correcting arc is $93 \mathrm{~m}$. From these numbers, it follows that this arc must have a negative momentum compaction of about -0.09 in order to offset the positive contributions from the rest of the ring.

The arc module is shown in Fig. 46. It has the small beta functions characteristic of FODO cells, yet a large, almost separate, variability in the momentum compaction of the module which is a characteristic associated with the flexible momentum compaction module [199,200]. The small beta functions are achieved through the use of a doublet focusing structure which produces a low beta simultaneously in both planes. At the dual minima, a strong focusing quadrupole is placed to control the derivative of dispersion with little impact on the beta functions. Negative values of momentum compaction as low as $\alpha=-0.13$ have been achieved, and $\gamma_{t}=2 i$ has been achieved with modest values of the beta function.
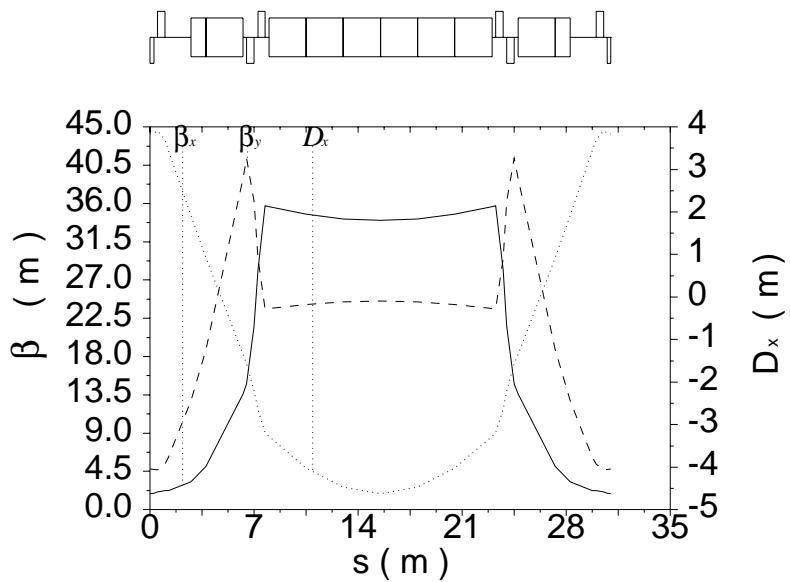

FIG. 46. Example (a): A flexible momentum compaction arc module.

This arc module was able to generate the needed negative momentum compaction with beta functions of $40 \mathrm{~m}$ or less.

A very preliminary calculation of the dynamic aperture [198] without optimization of the lattice or inclusion of errors and end effects is given in Fig. 47. One would expect that simply turning off the chromatic correction sextupoles in the $4 \mathrm{~cm} \beta^{*}$ mode would result in a linear lattice with a large transverse aperture. With only linear elements, the $4 \mathrm{~cm} \beta^{*}$ optics was found to be strongly nonlinear with limited on-momentum dynamic acceptance.

A normal form analysis using COSY INFINITY [201] showed that the variation of tune shift with amplitude was large, which was the source of the strong nonlinearity in the seemingly linear lattice. To locate the source of this nonlinearity, a lattice consisting of the original IR and arcs only (no CC) was studied. Numerical studies confirmed similar dynamic aperture and variation of tune shift with amplitude. This ruled out the possibility that the dynamic aperture was limited by the low beta points in the local chromatic correction section and points to the IR as the source of the nonlinearity. These findings were also verified [202] using a Runge-Kutta integrator to track through the IR and a linear matrix for the rest of the lattice. Further analytical study using perturbation theory showed that the first-order contribution to the tune shift with amplitude is proportional to $\gamma_{x, y}^{2}$ and $\gamma_{x} \gamma_{y}$, which are large in this IR. These terms come from the nonlinear terms of $p_{x} / p_{0}$ and $p_{y} / p_{0}$, which, to first order, equal the angular divergence of a particle. As a demonstration, a comparison to the LHC low beta IR was done. Taking into account only the drift from the IP to the first quadrupole, the horizontal detuning at $10 \sigma$ of the present IR $\left(\beta^{*}=4 \mathrm{~cm}\right)$ is 0.01 , whereas the detuning of the entire LHC lattice is below $1 E-$ 4. This also explains the fact that the on-momentum aperture of the wide momentum spread mode remains 


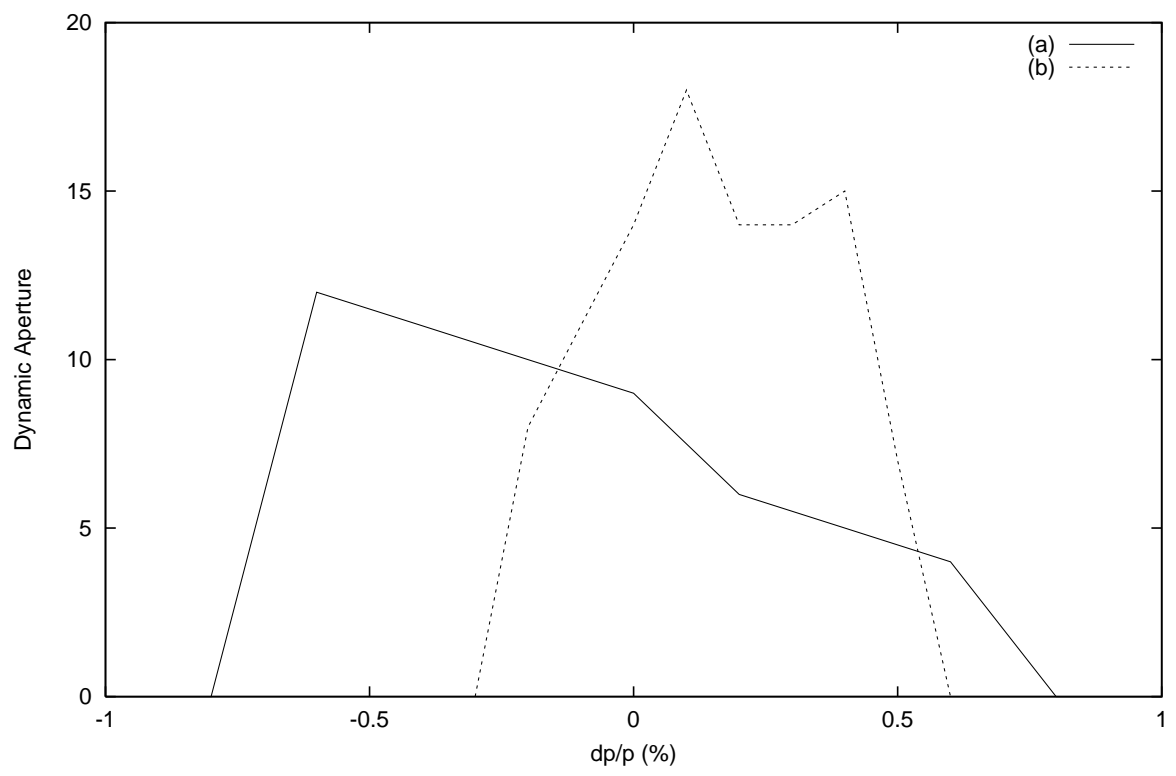

FIG. 47. Example (a): A preliminary dynamic aperture for the $4 \mathrm{~cm} \beta^{*}$ mode where $\sigma_{\text {rms }}=82 \mu \mathrm{m}$ (solid line) and the $14 \mathrm{~cm}$ $\beta^{*}$ mode where $\sigma_{\mathrm{rms}}=281 \mu \mathrm{m}$ (dashed line).

roughly constant despite various versions and correction attempts.

It was therefore concluded and later shown that the dynamic aperture of the more relaxed $\beta^{*}$ of $14 \mathrm{~cm}$ would not have the same strong nonlinearities due to the reduced angular terms. In fact, the variation of tune shift with amplitude was less by an order of magnitude; hence, the large transverse acceptance shown in Fig. 47 (dashed line).

\section{6. $100 \mathrm{GeV} \mathrm{COM}-$ Example (b)}

The second lattice design, Example (b), is shown in Fig. 48 starting from the IP. The $1.5 \mathrm{~m}$ background clearing dipole is $2.5 \mathrm{~m}$ away from the IP and is followed by the triplet quadrupoles with the focusing quadrupole in the center. The interaction region stops at about $24 \mathrm{~m}$ from the IP. Because of the small low betatron functions in both transverse planes, the betatron functions at the final focusing triplets increase to $\sim 1550 \mathrm{~m}$. The natural chromaticities, of order $\sim-40$, are high, requiring local correction. Due to the size limitation of the collider ring, it appears that we have room for only two pairs of interleaved sextupoles on each side of the IP, each pair correcting chromaticity in one transverse plane. The correction section on each side of the IP spans a distance of roughly $61.3 \mathrm{~m}$.

The SX1's are the two horizontal correction sextupoles. They should be placed at positions with the same betatron functions and dispersion function, and separated horizontally and vertically by phase advances $\Delta \psi_{x}$ and $\Delta \psi_{y}=\pi$ so that their nonlinear effect will be confined in the region between the two sextupoles. Their horizon- tal phase advances should also be integral numbers of $\pi$ from the triplet focusing $F$-quadrupole so that the chromaticity compensation for that quadrupole will be most efficient [197]. The SX2's are the two vertical correction sextupoles which should be placed similarly at designated locations. In general, it will be difficult to satisfy all the requirements mentioned; especially in this situation, luminosity arguments limit the lattice size. For this lattice, the Twiss properties at the centers of the four correction sextupoles are listed in Table XII, where all the figures given by the lattice code are displayed. An attempt was made to satisfy all the requirements at the expense of having $\Delta \psi_{y} /(2 \pi)=0.60$ instead of 0.50 for the SX1's. This trade-off is explained below.

The second-order effects of the sextupoles contribute to the amplitude dependent tune spreads, which, if too large, can encompass resonances leading to dynamical aperture limitation. For example, in this lattice,

$$
\begin{aligned}
& \nu_{x}=8.126337-100 \epsilon_{x}-4140 \epsilon_{y}, \\
& \nu_{y}=6.239988-4140 \epsilon_{x}-54.6 \epsilon_{y},
\end{aligned}
$$

where $\epsilon_{x}$ and $\epsilon_{y}$ are the horizontal and vertical unnormalized emittances in $\pi \mathrm{m}$. In order to eliminate these tune spreads due to the sextupole nonlinearity, the sufficient conditions are [203]

$$
\begin{array}{lll}
\sum_{k} \frac{S_{k} e^{i \psi_{x k}}}{\sin \pi \nu_{x}}=0, & \sum_{k} \frac{S_{k} e^{i 3 \psi_{x k}}}{\sin 3 \pi \nu_{x}}=0, \\
\sum_{k} \frac{\bar{S}_{k} e^{i \psi_{x k}}}{\sin \pi \nu_{x}}=0, & \sum_{k} \frac{\bar{S}_{k} e^{i \psi_{ \pm k}}}{\sin \pi \nu_{ \pm}}=0,
\end{array}
$$




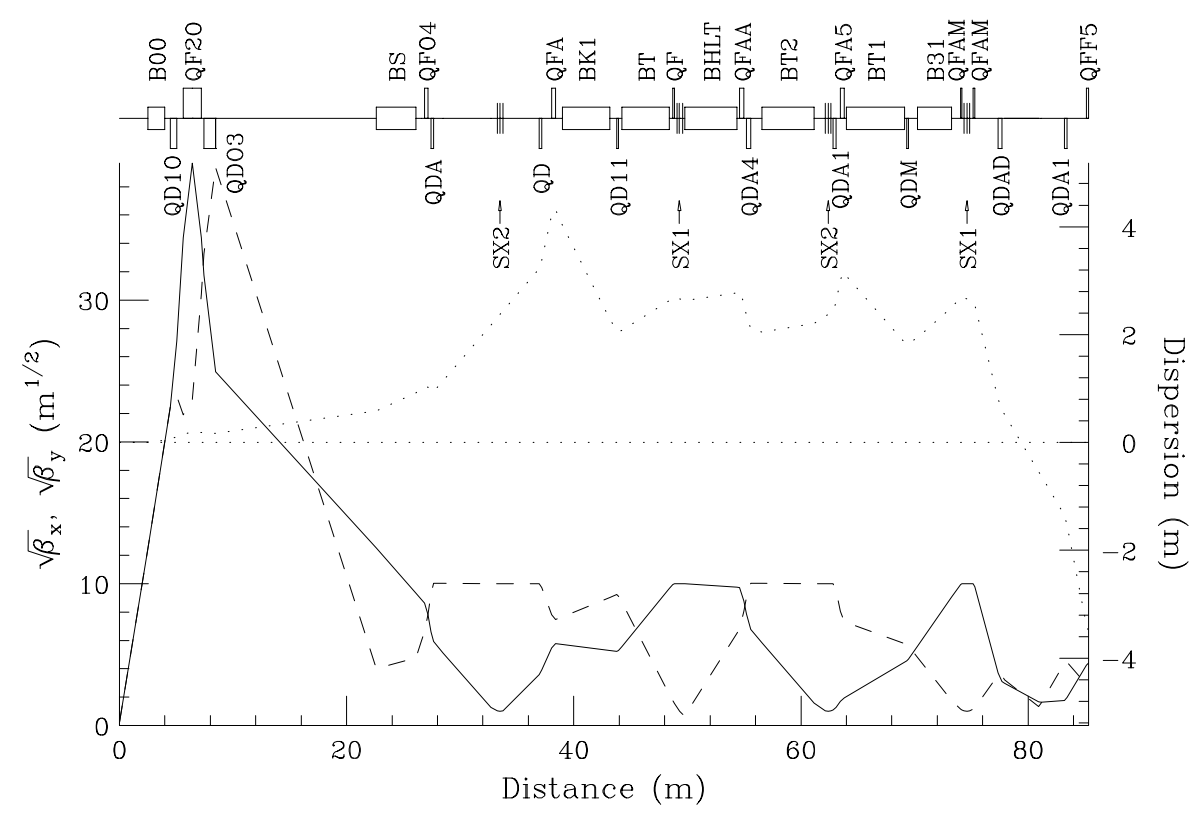

FIG. 48. Example (b): Lattice structure of the IR including local chromaticity corrections. $\beta_{x}$ (solid line), $\beta_{y}$ (dashed line), and dispersion (dotted line). The maximum and minimum $\beta_{x}$ are 1571.74 and $0.040 \mathrm{~m}$, the maximum and minimum $\beta_{y}$ are 1550.94 and $0.040 \mathrm{~m}$, while the maximum and minimum dispersions are 4.31 and $-3.50 \mathrm{~m}$. The natural horizontal and vertical chromaticities are -41.46 and -39.90 , giving a transition gamma of $\gamma_{t}=5.52$. The total module length is $85.32 \mathrm{~m}$ with a total bend angle of $1.307 \mathrm{rad}$.

where for the $k$ th thin normal sextupole with strength $S_{N k}=\lim _{\ell \rightarrow 0}\left[B^{\prime \prime} \ell /(B \rho)\right]_{k}$,

$$
S_{k}=\left[S_{N} \beta_{x}^{3 / 2}\right]_{k}, \quad \bar{S}_{k}=\left[S_{N} \beta_{x}^{1 / 2} \beta_{y}\right]_{k},
$$

$\psi_{ \pm k}=\left(2 \psi_{y} \pm \psi_{x}\right)_{k}$ and $\nu_{ \pm k}=\left(2 \nu_{y} \pm \nu_{x}\right)_{k}$. The five requirements come about because there are 5 first-order resonances driven by the sextupoles when the residual tunes of the ring satisfy $\left[3 \nu_{x}\right]=0,\left[\nu_{ \pm}\right]=0$, and two $\left[\nu_{x}\right]=0$. The nominal tunes shown in Eq. (28) are far from these resonances. Therefore, the sines in the denominators of Eq. (29) can be omitted in this discussion. Since the strengths of SX1 and SX2 are similar, we have $S_{\mathrm{SX} 2} \ll \bar{S}_{\mathrm{SX} 1} \ll \bar{S}_{\mathrm{SX} 2} \ll S_{\mathrm{SX} 1}$. In fact, they are roughly in the ratios of $1:\left(\beta_{\max } / \beta_{\min }\right)^{1 / 2}: \beta_{\max } / \beta_{\min }$ : $\left(\beta_{\max } / \beta_{\min }\right)^{3 / 2}$, which amount roughly to $1: 10: 100: 1000$ in this lattice. Above, $\beta_{\max }$ represents either $\beta_{x}$ at the SX1's or $\beta_{y}$ at the SX2's, and $\beta_{\min }$ represents either $\beta_{y}$ at the SX1's or $\beta_{x}$ at the SX2's. Thus, the first two restrictions in Eq. (29) are the most important, implying that all $\beta_{\max }$ and $\beta_{\min }$ for each pair of SX1's must be made equal and $\Delta \psi_{x}=\pi$ between them must be strictly obeyed. The third restriction is the next important one, for which $\bar{S}_{\mathrm{SX} 2}$ must be made equal for each pair of SX2's and their horizontal phase difference must equal $\pi$. The only two parameters left are $\Delta \psi_{y}$ between a pair of SX1's and $\Delta \psi_{y}$ between a pair of SX2's. They affect the restrictions for the $\nu_{ \pm}$resonances only, where the effective sextupole strengths $\bar{S}_{\mathrm{SX} 1}$ and $\bar{S}_{\mathrm{SX} 2}$ are involved. Thus if we allow one restriction to be relaxed, the relaxation of $\Delta \psi_{y}=\pi$ for the SX1's will be least harmful.

Flexible momentum compaction modules [200] are used in the arc. The momentum compaction of the arc has to be made negative in order to cancel the positive momentum compaction of the IR, so that the whole ring becomes quasi-isochronous. This is accomplished in three ways: (i) removing the central dipole of the usual flexible momentum compaction module, (ii) increasing the length of the first and last dipoles, and (iii) increasing the negative dispersion at the entrance. Two such modules will be required for half of the collider ring, one of which is shown in Fig. 49. To close the ring geometrically, there

TABLE XII. Twiss properties of the IR correction sextupoles.

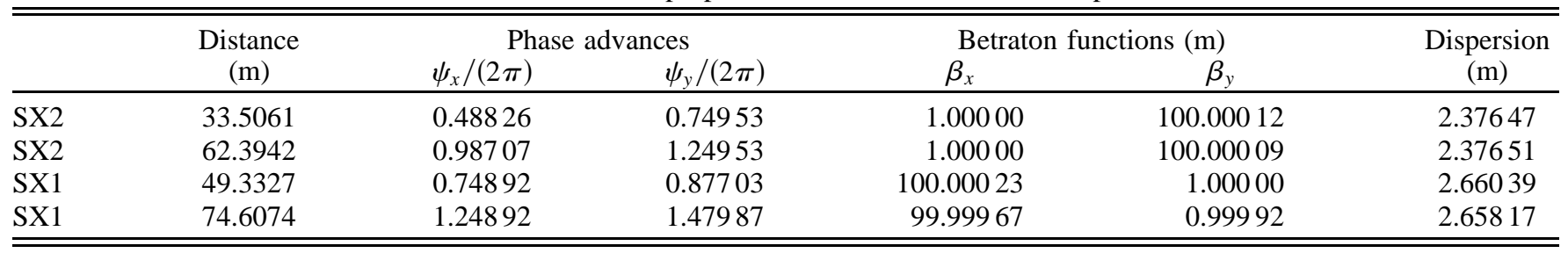




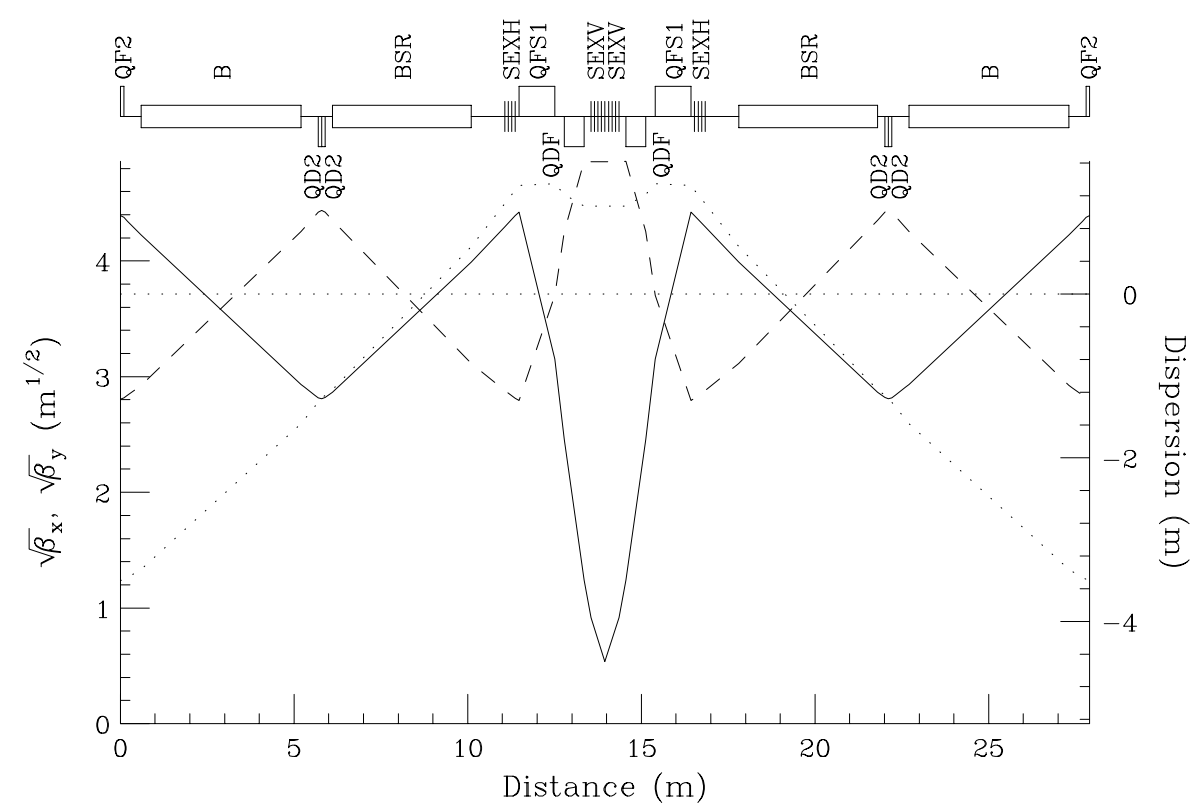

FIG. 49. Example (b): Lattice structure of the flexible momentum compaction module. $\beta_{x}$ (solid line), $\beta_{y}$ (dashed line), and dispersion (dotted line). The maximum and minimum $\beta_{x}$ are 19.57 and $0.29 \mathrm{~m}$, the maximum and minimum $\beta_{y}$ are 23.63 and $7.80 \mathrm{~m}$, the maximum and minimum dispersions are 1.35 and $-3.50 \mathrm{~m}$. The natural horizontal and vertical chromaticities are -1.77 and -0.92 , giving a transition gamma of $\gamma_{t}=i 4.43$. The total module length is $27.91 \mathrm{~m}$ with a total bend angle of $0.917 \mathrm{rad}$.

will be a $\sim 72.0 \mathrm{~m}$ straight section between the two sets of flexible momentum compaction modules. The total length of the collider ring is now only $C=354.3 \mathrm{~m}$. This is a nice feature, since a small ring allows a larger number of collisions before the muons decay appreciably. Note that the IR and local correction sections take up $48.2 \%$ of the whole ring. The momentum compaction factor of this ring is now $\alpha_{0}=-2.77 \times 10^{-4}$. The rf voltage required to maintain a bunch with rms length $\sigma_{\ell}$ and rms momentum spread $\sigma_{\delta}$ is $V_{\mathrm{rf}}=|\eta| E C^{2} \sigma_{\delta}^{2} /\left(2 \pi h \sigma_{\ell}^{2}\right)$, where $\eta$ is the slippage factor and $E$ is the muon energy. On the other hand, if the bucket height is taken as $k$ times the rms momentum spread of the bunch, the rf harmonic is given by $h=C /\left(k \pi \sigma_{\ell}\right)$. Thus, for $\sigma_{\ell}=4 \mathrm{~cm}$ and $\sigma_{\delta}=0.0012$, this lattice requires an $\mathrm{rf}$ voltage of $V_{\mathrm{rf}} \approx 88 \mathrm{k} \mathrm{kV}$. Since $\alpha_{0}$ is negative already, its absolute value can be further lowered easily if needed. However, we must make sure that the contributions from the higher order momentum compaction are small in addition.

The dynamical aperture of the lattice is computed by tracking particles with the code COSY INFINITY [201]. Initially, 16 particles with the same momentum offset and having vanishing $x^{\prime}$ and $y^{\prime}$ are placed uniformly on a circle in the $x-y$ plane. The largest radius that provides survival of the 16 particles in 1000 turns is defined here as the dynamical aperture at this momentum offset and is plotted in solid lines in Fig. 50 in units of the rms radius of the beam. (At the $4 \mathrm{~cm}$ low beta IP, the beam has an rms radius of $82 \mu \mathrm{m}$.) As a reference, the $7 \sigma$ aperture spanning $\pm 6 \sigma$ of momentum offset is also displayed as a semiellipse in dot-dashed lines. To maximize the aperture, first, the tunes must be chosen to avoid parametric resonances. The onmomentum amplitude dependent horizontal and vertical tunes are given in Eq. (28). With the designed rms $\epsilon_{x}=\epsilon_{y}=0.169 \times 10^{-6} \pi \mathrm{m}$, the on-momentum tune variations are at most 0.0007 . Second, the chromaticity variations with momentum must be as small as possible. This is shown in Fig. 50 (right-hand-side plot). Note that there are no families of sextupoles to correct for the higher order chromaticities in this small ring with only four flexible momentum compaction modules. As the momentum spread varies from $-1 \%$ to $0.9 \%, \nu_{x}$ varies from 8.16698 to 8.07459 , and $\nu_{y}$ from 6.28305 to 6.22369 for the center of the beam.

During aperture tracking we notice that particle loss occurs mostly in the horizontal direction. We are convinced that the small momentum aperture is a result of the large dispersion swing in the lattice from +4.5 to $-3.5 \mathrm{~m}$. For example, $4.5 \mathrm{~m}$ dispersion and $0.6 \% \mathrm{mo}-$ mentum offset translates into a $2.7 \mathrm{~cm}$ off axis motion. The nonlinearity of the lattice will therefore diminish the dynamical aperture. A resonant strength study using, for example, swamp plots and normalized-resonance-basiscoefficient analysis [204] actually reveals that this lattice and some of its variations are unusually nonlinear. Recently, we made a modification of the flexible momentum compaction arc modules which have a smaller dispersion swing from -2.6 to $+2.0 \mathrm{~m}$ only. The IR has not been changed except for the matching to the arc modules. The aperture has been tracked with TEAPOT [205] in the same way as COSY and is plotted as a dashed line in Fig. 50 

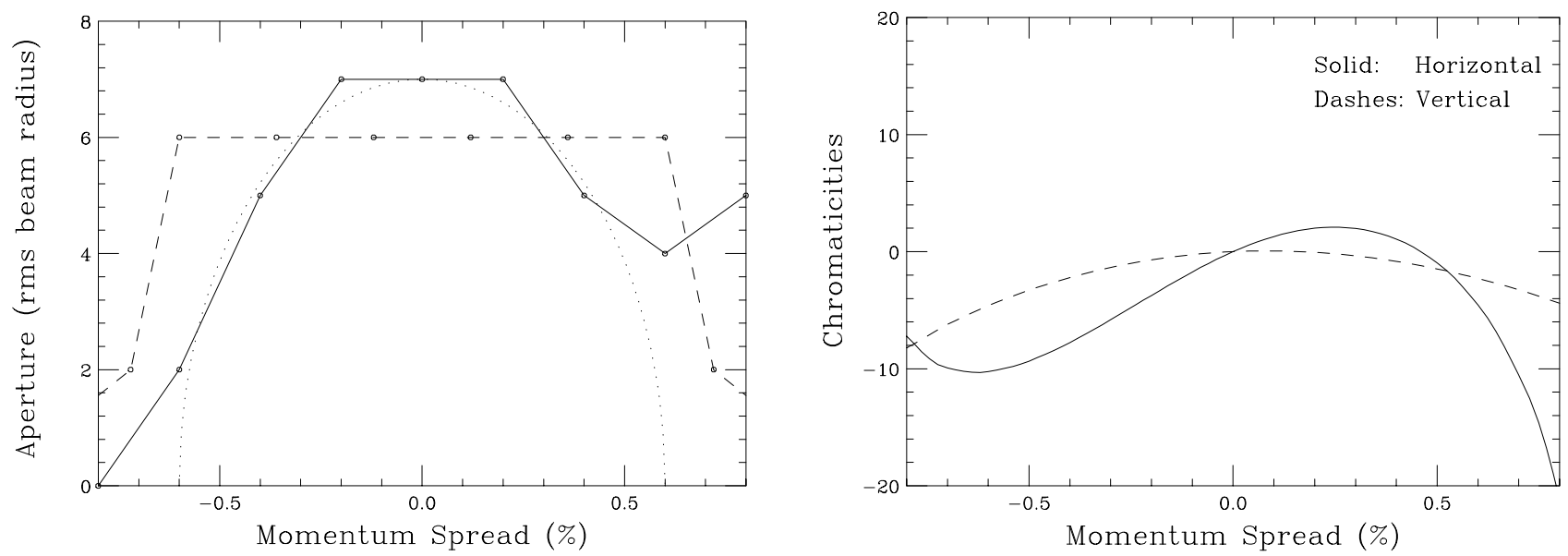

FIG. 50. Example (b): Left-hand-side plot is dynamical aperture of the lattice vs momentum offset. COSY calculation in solid line, $7 \sigma_{\mathrm{rms}}$ in dot-dashed line, and TEAPOT calculation with modified FMC modules in dashed line. Right-hand-side plot is chromaticities vs momentum offset.

(left-hand-side plot). We see that the momentum aperture has widened appreciably. The dynamical aperture near on-momentum, however, is $1 \sigma$ less than the lattice presented here. Nevertheless, it is not clear that this decrease is significant because all tracking has been performed in steps of $1 \sigma$ only. However, this type of aperture is still far from satisfactory, because so far we have been studying a bare lattice. The aperture will be reduced when fringe fields, field errors, and misalignment errors are included.

We suspect that the aperture for small momentum spread is limited by the dramatic changes in betatron functions near the IP [202]. These changes are so large that Hill's equation would no longer be adequate and the exact equation for beam transport must be used. This equation brings in nonlinearity and limits the aperture, which can easily be demonstrated by turning off all the sextupoles. In other words, although the momentum aperture can be widened by suitable deployment of sextupoles, the on-momentum dynamical aperture is determined by the triplet quadrupoles and cannot be increased significantly by the sextupoles. Some drastic changes in the low beta design may be necessary.

\section{Scraping}

It has been shown [206] that detector backgrounds originating from beam halo can exceed those from decays in the vicinity of the interaction point. Only with a dedicated beam cleaning system far enough from the IP can one mitigate this problem [196]. Muons injected with large momentum errors or betatron oscillations will be lost within the first few turns. After that, with active scraping, the beam halo generated through beam-gas scattering, resonances, and beam-beam interactions at the IP reaches equilibrium and beam losses remain constant throughout the rest of the cycle.
Two beam cleaning schemes have been designed [196], one for muon colliders at high energies, and one for those at low energies.

The studies [196] showed that no absorber, ordinary or magnetized, will suffice for beam cleaning at $2 \mathrm{TeV}$; in fact, the disturbed muons are often lost in the IR, but a simple metal collimator was found to be satisfactory at $100 \mathrm{GeV}$.

\section{Scraping for high energy collider}

At high energies, a $3 \mathrm{~m}$ long electrostatic deflector (Fig. 51) separates muons with amplitudes larger than $3 \sigma$ and deflects them into a $3 \mathrm{~m}$ long Lambertson magnet, which extracts these downward through a deflection of $17 \mathrm{mrad}$. A vertical septum magnet is used in the vertical scraping section instead of the Lambertson to keep the direction of extracted beam down. The shaving process lasts for the first few turns. To achieve practical distances and design apertures for the separator/Lambertson combinations, $\beta$ functions must reach $1 \mathrm{~km}$ in the $2 \mathrm{TeV}$ case, but only $100 \mathrm{~m}$ at $50 \mathrm{GeV}$. The complete system consists of a vertical scraping section and two horizontal ones for positive and negative momentum scraping (the design is symmetric about the center, so scraping is identical for both $\mu^{+}$and $\mu^{-}$). The system provides the scraping power of a factor of 1000; that is, for every 1000 halo muons, one remains.

\section{Scraping for low energy collider}

At $50 \mathrm{GeV}$, collimating muon halos with a $5 \mathrm{~m}$ long steel absorber (Fig. 52) in a simple compact utility section do an excellent job. Muons lose a significant fraction of their energy in such an absorber ( $8 \%$ on average) and have broad angular and spatial distributions. Almost all of these muons are then lost in the first 50-100 m downstream 


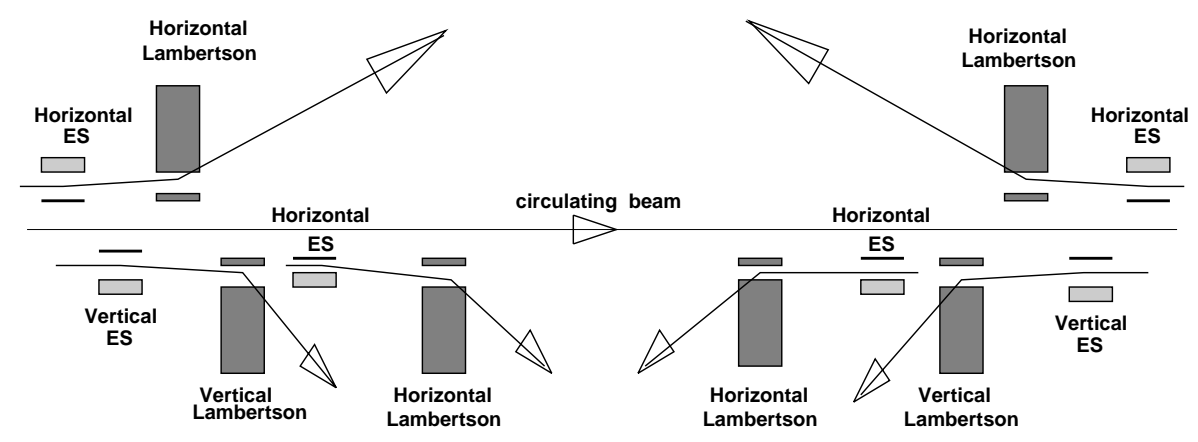

FIG. 51. Schematic view of a $\mu^{+} \mu^{-}$collider beam halo extraction.

of the absorber with only $0.07 \%$ of the scraped muons reaching the low $\beta$ quadrupoles in the IR, i.e., a scraping power is 1500 in this case, which is significantly better than with an earlier septum scraping system design [196] similar to that developed for the high energy collider.

\section{Beam-beam tune shift}

Several studies have considered beam emittance growth due to the beam-beam tune shift and none have observed significant luminosity loss. For instance, a study [207] using the high energy collider parameters (see Table I), in which particles were tracked assuming Gaussian beam field distributions and no muon decay, showed a luminosity loss of only $4 \%$. With muon decay included, the loss contribution from beam-beam effects is even less. Another study [208] using a particle in cell approach with no assumptions about field symmetry obtained a similar result. Collisions between beams displaced by $10 \%$ of their radius also gave little loss. But all these studies assumed an ideal lattice, and none considered whether small losses due to nonlinearities give rise to an unacceptable background.

\section{E. Impedance/wake field considerations}

A study [209] has examined the resistive wall impedance longitudinal instabilities in rings at several energies. At the higher energies and larger momentum spreads, solutions were found with small but finite momentum compaction and moderate rf voltages. For the special case of the Higgs factory, with its very low momentum spread, a solution was found with no synchrotron motion, but rf was provided to correct the first-order impedance generated momentum spread. The remaining off-momentum tails which might generate background could be removed by a higher harmonic rf correction without affecting luminosity. Solutions to the higher energy and larger momentum spread cases without synchrotron motion are also being considered.

Given the very slow or nonexistent synchrotron oscillations, the transverse beam breakup instability is significant. This instability can be stabilized using rf quadrupole [189] induced BNS damping. For instance, the required tune shift with position in the bunch, calculated using the two particle model approximation [210], is only $1.58 \times 10^{-4}$ for the $3 \mathrm{TeV}$ case using a $1 \mathrm{~cm}$ radius aluminum pipe. This stabilizes the resistive wall instability. However, this application of BNS damping to a quasiisochronous ring, and other head-tail instabilities due to the chromaticities $\xi$ and $\eta_{1}$, needs more study.

\section{F. Bending magnet design}

The dipole field assumed in the $100 \mathrm{GeV}$ collider lattices described above was $8 \mathrm{~T}$. This field can be obtained



FIG. 52. Scraping muon beam halo with a $5 \mathrm{~m}$ steel absorber. 




FIG. 53. (Color) Power penetrating tungsten shields vs their thickness for (a) $4 \mathrm{TeV}$ and (b) $0.1 \mathrm{TeV}$ colliders.

using $1.8 \mathrm{~K}$ niobium titanium ( $\mathrm{NbTi}$ ) cos theta superconducting magnets similar to those developed for the LHC. The only complication is the need for a tungsten shield between the beam and the coils to shield the latter from beam decay heating.

The $\mu$ 's decay within the rings $\left(\mu^{-} \rightarrow e^{-} \overline{\nu_{e}} \nu_{\mu}\right)$, producing electrons whose mean energy is approximately 0.35 that of the muons. With no shielding, the average power deposited per unit length would be about $2 \mathrm{~kW} / \mathrm{m}$ in the $4 \mathrm{TeV}$ machine, and $300 \mathrm{~W} / \mathrm{m}$ in the $100 \mathrm{GeV}$ Higgs factory. Figure 53 shows the power penetrating tungsten shields of different thickness [44,195,196,211]. One sees that $3 \mathrm{~cm}$ in the low energy case, or $6 \mathrm{~cm}$ at high energy, would reduce the power to below $10 \mathrm{~W} / \mathrm{m}$, which can reasonably be taken by superconducting magnets.

Figure 54 shows the cross section of a baseline magnet suitable for the $100 \mathrm{GeV}$ collider.

The quadrupoles could use warm iron poles placed as close to the beam as practical. The coils could then be either superconducting or warm, placed at a greater distance from the beam and shielded from it by the poles.

The collider ring could be made smaller, and the luminosity increased, if higher field dipoles were used. In the low energy case, the gain would not be great since less than half of the circumference is devoted to the arcs. For this reason, and to avoid yet another technical challenge, higher field magnets are not part of the baseline design of a $100 \mathrm{GeV}$ collider. But they would give a significant luminosity improvement for the higher energy colliders, and would be desirable there. There have been several studies of possible designs, three of which (two that are promising and one that appears not to work) are included below.

\section{Alternative racetrack $\mathrm{Nb}_{3} \mathrm{Sn}$ dipole}

A higher field magnet based on $\mathrm{Nb}_{3} \mathrm{Sn}$ conductor and racetrack coils is presently being designed. The $\mathrm{Nb}_{3} \mathrm{Sn}$ conductor allows higher fields and provides a large temperature margin over the operating temperature, but, being brittle and sensitive to bending or other stress, presents a number of engineering challenges.

In this design, the stress levels in the conductor are reduced by the use of a rectangular coil block geometry, and end support problems are reduced by keeping the coils flat. In the more conventional cos theta designs, the conductor is distributed around a cylinder and the forces add up toward the midplane; in addition, the ends, as they arc over the cylinder, are relatively hard to support.

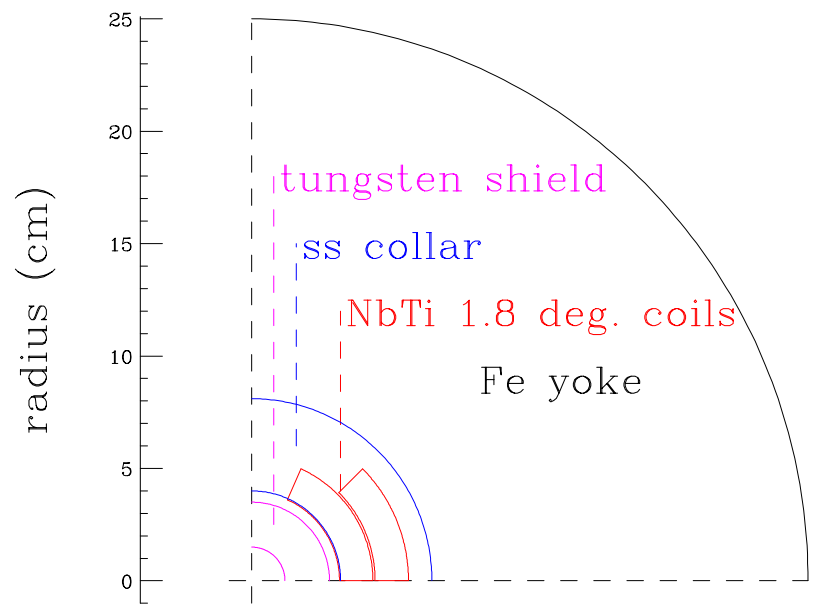

FIG. 54. (Color) Cross section of a baseline dipole magnet suitable for the $100 \mathrm{GeV}$ collider. 


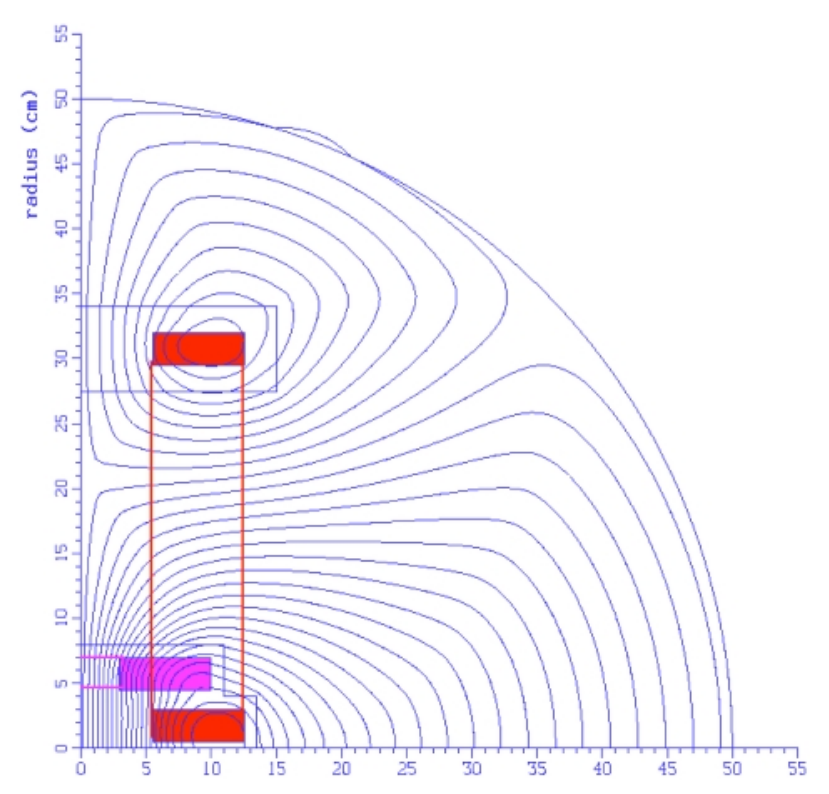

FIG. 55. (Color) Cross section of alternative high field $(\approx 15 \mathrm{~T})$ racetrack coil dipole magnet with $\mathrm{Nb}_{3} \mathrm{Sn}$ conductor.

The geometry of the cross section is shown in Fig. 55 . It uses all 2D flat racetrack coils. Each quadrant of the magnet aperture has two blocks of conductors. The block at the pole in the first quadrant has a return block in the second quadrant, similar to that in a conventional design. The height of this block is such that it completely clears the bore. In a conventional design, the second block, the midplane block, would also have a return block in the second quadrant. That would, however, require the conductor block to be lifted up in the ends to clear the bore and thus would lose the simple 2D geometry. In the proposed design, the return block retains the 2D coil geometry, as it is returned on the same side (see Fig. 55) and naturally clears the bore. Since the return block does not contribute to the field, this design uses 50\% more conductor. This, how- ever, is a small penalty to pay for a few magnets where the performance and not the cost is a major issue. The field lines are also shown in Fig. 55.

Preliminary design parameters for two cases are given in Table XIII. The first case is one where the performance of the cable used is the same that is in the LBL D20 magnet, which created a central field of $13.5 \mathrm{~T}$. The second case is the one where the cable is graded and two types of cable are used, and it is assumed that a reported improvement in cable performance is realized. It is expected to produce a central field of $14.7 \mathrm{~T}$ when operated at $42 \mathrm{~K}$.

\section{Alternative cos theta $\mathrm{Nb}_{3} \mathrm{Sn}$ dipole}

In this case the problem with the brittle and sensitive conductor is solved by winding the coil inside many separate slots cut in metal support cylinders. There is no buildup of forces on the coil at the midplane. The slots continue around the ends, and thus solve the support problem there too.

Figure 56 shows this alternative $\mathrm{Nb}_{3} \mathrm{Sn}$ dipole cos theta design. It is an extension of the concept used to build helical magnets [212] for the polarized proton program at RHIC [213]. The magnet is wound with prereacted, kapton-insulated, $B$-stage impregnated, low current cable. The buildup of forces is controlled by laying the cables in machined slots in a metal support cylinder. After winding, the openings of the slots are bridged by metal spacers and the coils precompressed inward by winding $B$-stage impregnated high tensile thread around the spacers. After curing, the outside of each coil assembly is machined prior to its insertion into an outer coil, or into the yoke. There are three layers. The inner bore is $55 \mathrm{~mm}$ radius, the outer coil radius approximately $118 \mathrm{~mm}$, and the yoke inside radius is $127 \mathrm{~mm}$. The maximum copper current density is $1300 \mathrm{~A} / \mathrm{mm}^{2}$.

TABLE XIII. Preliminary design parameters for a racetrack $\mathrm{Nb}_{3}$ Sn dipole with two different types of cable.

Case 1: Same conductor as in LBL 13.5 T D20 magnet without grading

Central field at quench

Coil dimensions

Total number of racetrack coils in whole magnet

Total number of blocks per quadrant in aperture

Yoke outer radius

Field harmonics

Midplane gap (midpalne to coil)

Minimum coil height in the end

Case 2: Newer conductor and graded

Central field at quench

Grading

Overall current densities

Peak fields

Copper current density

Other features are the same as in Case 1
$14.7 \mathrm{~T}$ at $4.2 \mathrm{~K}$

$70 \mathrm{~mm}$ divided in two $35 \mathrm{~mm}$ layers $370 \mathrm{~A} / \mathrm{mm}^{2}$ and $600 \mathrm{~A} / \mathrm{mm}^{2}$

16 and $12.5 \mathrm{~T}$

$1500 \mathrm{~A} / \mathrm{mm}^{2}$ 


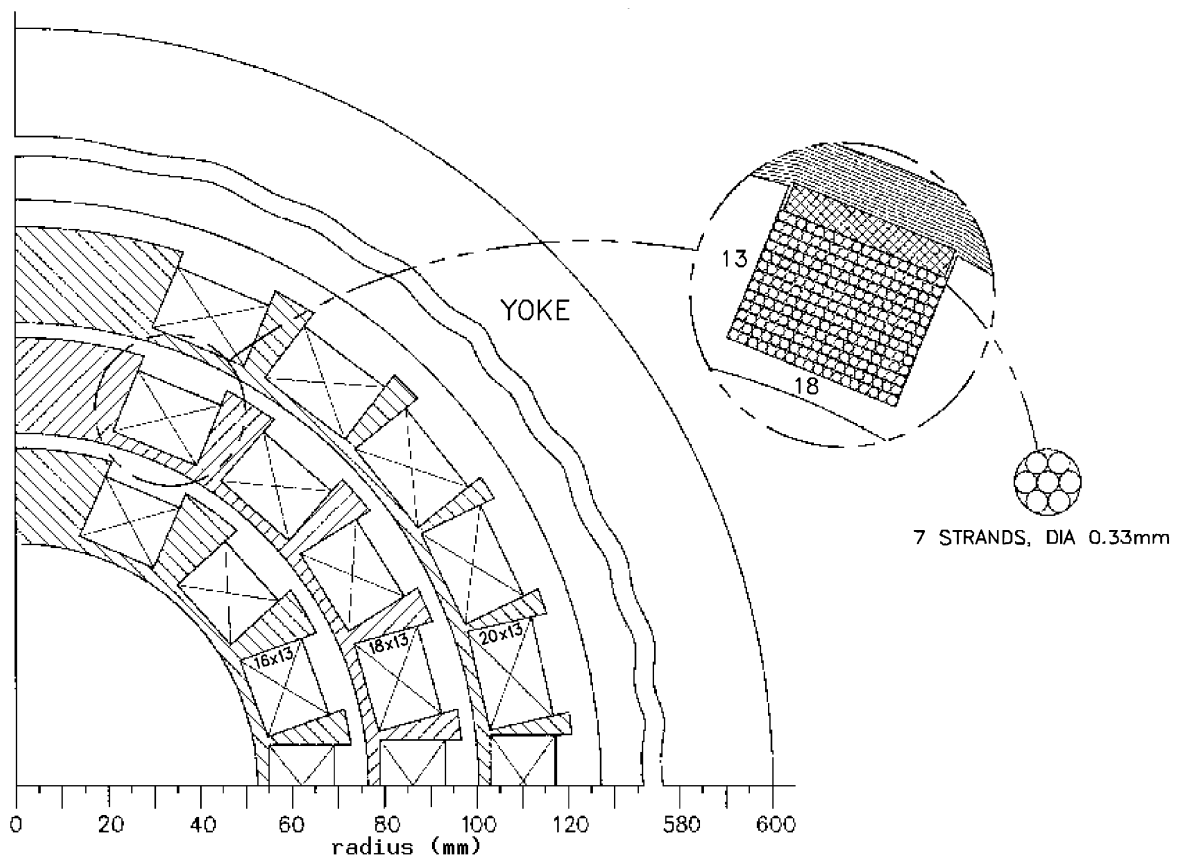

FIG. 56. Cross section of alternative high field slot dipole made with $\mathrm{Nb}_{3} \mathrm{Sn}$ conductor.

Using the same material specifications as used in the above high field option, a central short sample field of 13.2 $\mathrm{T}$ was calculated. This is somewhat less than the block design discussed above, but could be improved by increasing the cable diameters to improve the currently rather poor $(64 \%)$ cable to cable-plus-insulator ratio.

\section{Study of C-magnet dipole}

Figure 57 shows the cross section of a high field dipole magnet in which it was hoped to bring the coils closer to the beam pipe without suffering excessive heating from beam decay. The coil design [214] appeared reasonable, but the required avoidance of coil heating was not achieved.

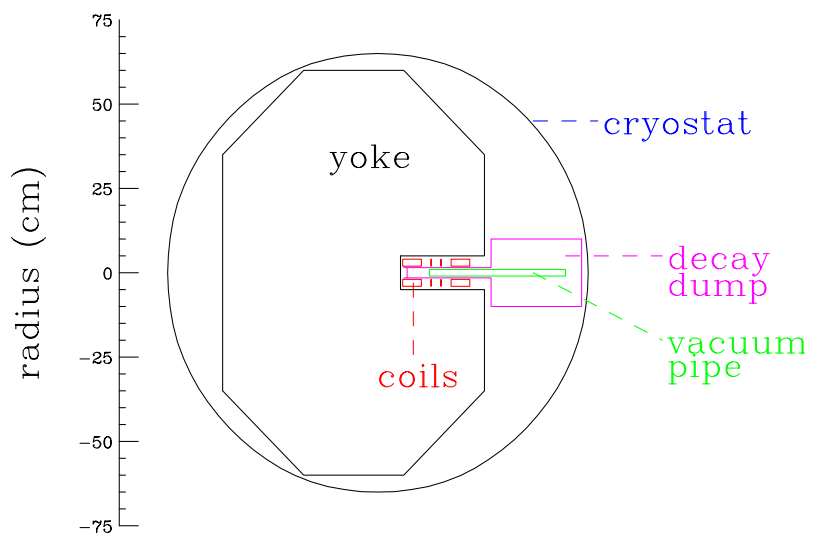

FIG. 57. (Color) Cross section of an unsuccessful alternative high field $C$ magnet with open midplane.
Decay electrons are generated at very small angles $(\approx 1 / \gamma)$ to the beam, and with an average energy about $1 / 3$ of the beam. Such electrons initially spiral inward (to the right in Fig. 57) bent by the high dipole field. In the high energy case, these electrons also radiate a significant fraction of their energy as $(\approx 1 \mathrm{GeV})$ synchrotron gamma rays, some of which end up on the outside (to the left in Fig. 57). The concept was to use a very wide beam pipe, allow the electrons to exit between the coils, and be absorbed in an external cooled dump. Unfortunately, a preliminary study found that a substantial fraction of the electrons did not reach the dump. They were bent back outward before reaching it by the return field of the magnet coils and the nature of the curved ring geometry. Such electrons were then trapped about the null in the vertical field and eventually hit the upper or lower face of the unshielded vacuum pipe. They showered, and deposited unacceptable levels of heat in the coils.

Another idea called for collimators between each bending magnet that would catch such trapped electrons. This option has not been studied in detail, but the impedance consequences of such periodic collimators are expected to be unacceptable.

Further study of such options might find a solution, but the use of a thick cylindrical heavy metal shield appears practical, adequate, and is thus the current baseline choice.

\section{G. Energy scale calibration}

In order to scan the width of a Higgs boson of mass around $100 \mathrm{GeV}$, one needs to measure the energy of the 
individual muon stores to an accuracy of a few parts per $10^{6}$, since the width of a Higgs boson of that mass is expected to be a few MeV. Assuming that muon bunches can be produced with modest polarizations of $\approx 0.25$, and that the polarization can be maintained from turn to turn in the collider, it is possible to use the precession of the polarization in the ring to measure accurately the average energy of the muons [12]. The total energy of electrons produced by muon decay observed in the calorimeter placed in the ring varies from turn to turn due to the $g-$ 2 precession of the muon spin, which is proportional to the Lorentz factor $\gamma$ of the muon beam. Figure 58 shows the result of a fit of the total electron energy observed in a calorimeter to a functional form that includes muon decay and spin precession. Figure 59(a) shows the fractional error $\delta \gamma / \gamma$ obtained from a series of such fits plotted against the fractional error of measurement in the total electron energy that depends on the electron statistics. It has been shown that precisions of a few parts per $10^{6}$ in $\gamma$ are possible with modest electron statistics of $\approx 100000$ detected. It should be noted that there are $3.2 \times 10^{6}$ decays per meter for a muon intensity of $10^{12}$ muons. Figure 59(b) shows the fractional error in $\delta \gamma / \gamma$ obtained by fitting the rate of decay of the muons in the collider. The accuracy using this method is much worse than that from fitting the polarization oscillation and cannot be used for precision measurement of the energy. Figure 59(c) shows the $\chi^{2}$ of the fits. No significant fitting biases are evident.

Our current plans to measure the energy due to decay electrons entail an electromagnetic calorimeter that is segmented both longitudinally and transversely and placed inside an enlarged beam pipe in one of the straight sections in the collider ring. The length of the straight section upstream of the calorimeter can be chosen to control the total number of decays and hence the rate of energy deposition. The sensitive material can be gaseous, since the energy resolution is controlled by decay fluctuations rather than sampling error. In order to measure the total number of electrons entering the calorimeter, we plan to include a calorimeter layer with little absorber upstream of it as the first layer.

This scheme will enable us to calibrate and correct the energy of individual bunches of muons and permit us to measure the width of a low mass Higgs boson.

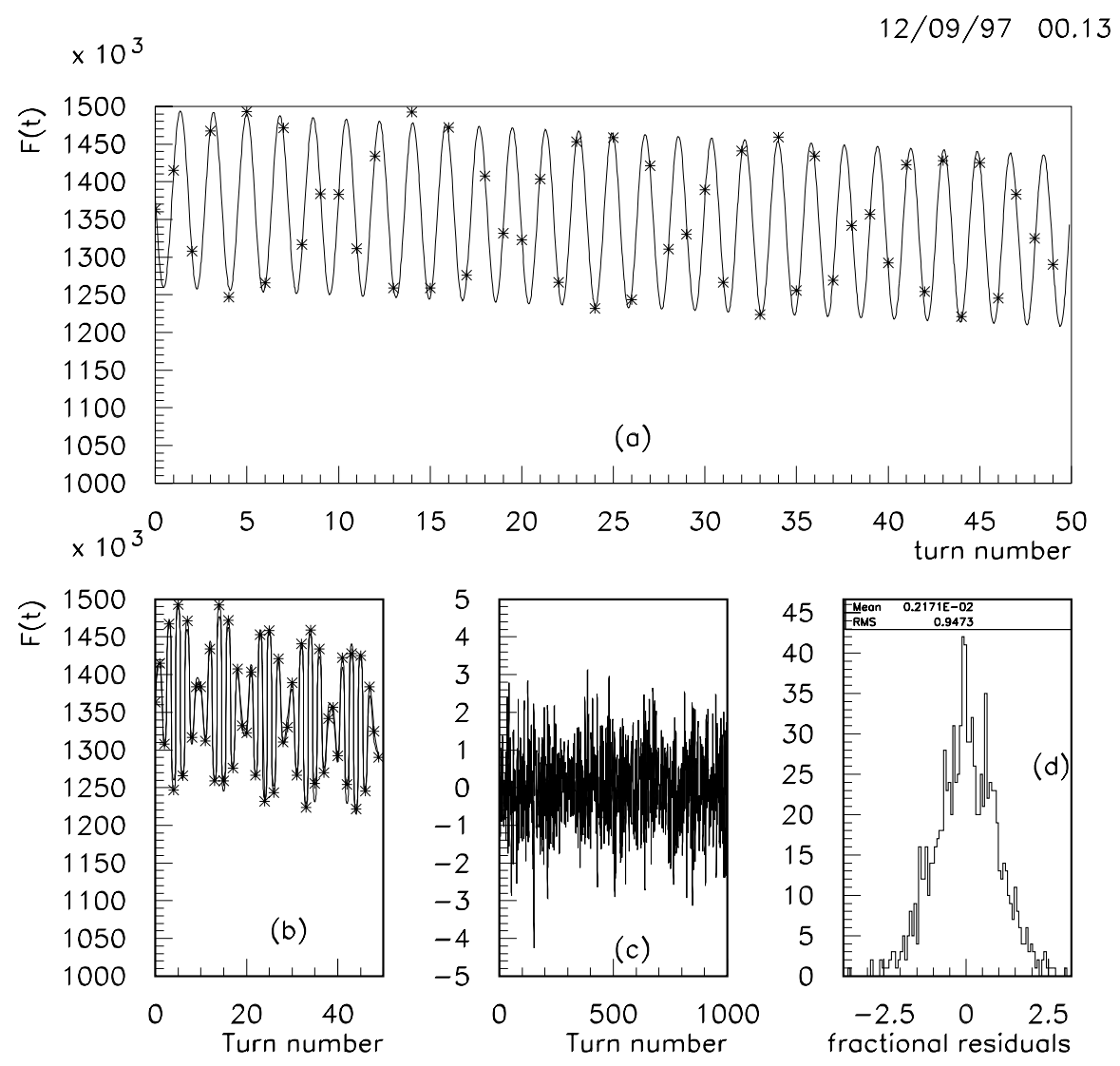

FIG. 58. (a) Energy detected in the calorimeter during the first 50 turns in a $50 \mathrm{GeV}$ muon storage ring (points). An average polarization value of $\hat{P}=-0.26$ is assumed and a fractional fluctuation of $5 \times 10^{-3}$ per point. The curve is the result of a MINUIT fit to the expected functional form. (b) The same fit, with the function being plotted only at integer turn values. A beat is evident. (c) Pulls as a function of turn number. (d) Histogram of pulls. A pull is defined by (measured value-fitted value)/(error in measured-fitted). 


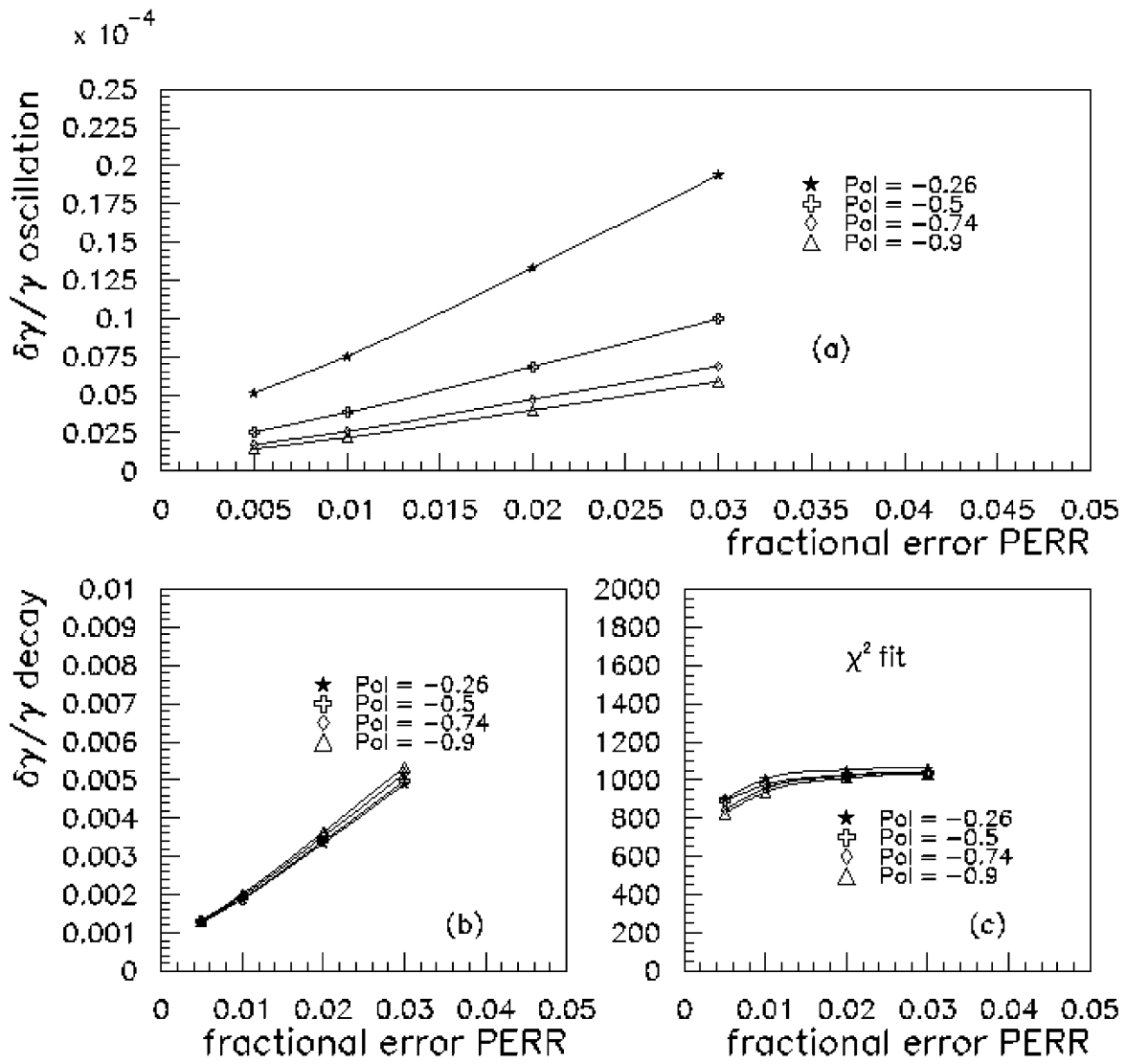

FIG. 59. (a) Fractional error in $\delta \gamma / \gamma$ obtained from the oscillations as a function of polarization $\hat{P}$ and the fractional error in the measurements PERR. (b) Fractional error in $\delta \gamma / \gamma$ obtained from the decay term as a function of polarization $\hat{P}$ and the fractional error in the measurements PERR. (c) The total $\chi^{2}$ of the fits for 1000 degrees of freedom. PERR is the percentage measurement error on the total electron energy in the calorimeter measuring the decay electrons.

\section{RADIATION AND BACKGROUNDS}

\section{A. Conventional radiation}

The proton source generates a 4 MW proton beam, which is comparable to the proposed spallation source [92]. This is a very high power and will, as in the spallation source, require great care in reducing unwanted particle losses, as well as careful machine shielding, and target and beam dump design. Initial studies of the target and capture solenoid region have been performed with the MARS code, and preliminary specifications for shielding determined, but more work is needed.

The cooling and accelerator chain is rather clean, since a relatively small fraction of the muons decay, and their energies are low. Power deposited in the accelerators is typically $10-30 \mathrm{~W} / \mathrm{m}$ (see Tables IX and X).

If no muons are lost, the only sources of radiation are the muon decays yielding electrons and neutrinos. The neutrino radiation will be discussed below. The electrons shower in the collider beam pipe shields, depositing most of their energy there and a relatively small amount in the magnet coils and yoke. Radioactivation levels, as calculated by MARS [215], after five years of $4 \mathrm{TeV}$ collider operation are given in Table XIV for the cases immediately after turn-off and one day after turn-off. It is seen that the areas in the tunnel that are outside the magnets are relatively free of radioactivation. Special procedures will be needed when the shield pipe has to be opened, as, for instance, when a magnet is changed. For the lower energy colliders, the radioactivation levels are proportionally less.

If muons are lost either accidentally, by scraping, or deliberately after some number of turns, then the muons penetrate to considerable distances in the soil/rock $(3.5 \mathrm{~km}$ at $2 \mathrm{TeV}, 800 \mathrm{~m}$ at $250 \mathrm{GeV}$ ) and deposit their energy directly or through their interaction products. Figures 60 and 61 show the distribution of radiation levels, assuming $25 \%$ of all muons (four bunches of $2 \times 10^{12}$ at $15 \mathrm{~Hz}$ )

TABLE XIV. $4 \mathrm{TeV}$ (COM) collider ring radioactivation levels $(\mathrm{mrem} / \mathrm{h})$ after turn-off, for parameters in Table I.

\begin{tabular}{lcc}
\hline \hline & Immediate & After one day \\
\hline Inside face of shield & 9000 & 4000 \\
Outside face of shield & 200 & 170 \\
Outside of coils & 30 & 14 \\
Outside of yoke & 3 & 1.4 \\
\hline \hline
\end{tabular}




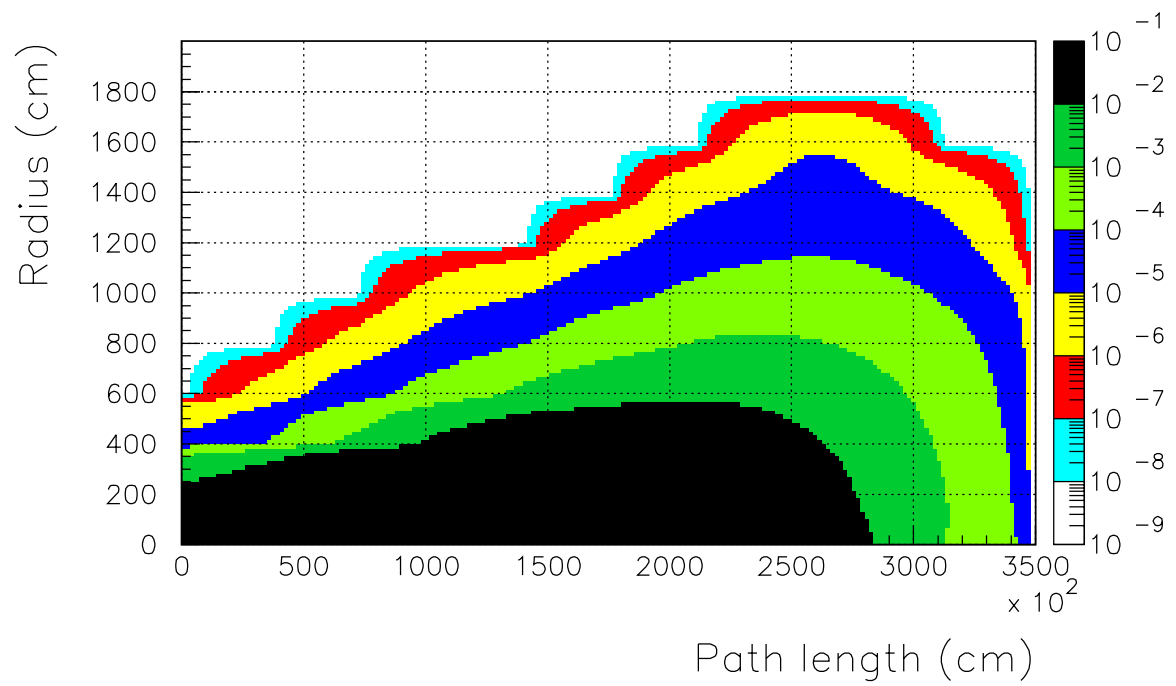

FIG. 60. (Color) Isodose contours in the soil/rock $\left(\rho=2.24 \mathrm{~g} / \mathrm{cm}^{3}\right)$ for $2 \mathrm{TeV}$ muons extracted at $3 \times 10^{13}$ per second. Righthand scale is dose rate in rem/s.

are dumped into soil/rock with density $2.24 \mathrm{~g} / \mathrm{cm}^{3}$. The outer contours correspond to the federal limits, reached at maximum radii of $18 \mathrm{~m}(2 \mathrm{TeV})$ and $14.5 \mathrm{~m}(250 \mathrm{GeV})$. To confine this radiation beneath the ground, one can deflect the extracted beams down by $4.5 \mathrm{mrad}$ at $2 \mathrm{TeV}$ and about $10 \mathrm{mrad}$ at $\leq 250 \mathrm{GeV}$. If any water were present in the soil/rock, then the first $2 \mathrm{~m}$ around the tunnel and around the aborted beam axis would require insulation or drainage up to a distance of $2.5 \mathrm{~km}$ at $2 \mathrm{TeV}$ or $550 \mathrm{~m}$ at $250 \mathrm{GeV}$.

\section{B. Neutrino induced radiation}

It has been shown [64,216-219] that the neutrinos created in muon beam decays can generate excessive secondary radiation at large distances from a muon collider (see Fig. 62). The surface radiation dose $D_{B}(\mathrm{~Sv})$ in units of equivalent [220] doses (Sv) over a time $t$ (s), in the plane of a bending magnet of field $B(\mathrm{~T})$, in a circular collider with beam energy $E(\mathrm{TeV})$, average bending field $\langle B\rangle(\mathrm{T})$, at a depth $d(\mathrm{~m})$ (assuming a spherical earth), with muon current (of each sign) of $I_{\mu}$ (muons/s/sign) is given by

$$
D_{B} \approx 4.4 \times 10^{-24} \frac{I_{\mu} E^{3} t}{d} \frac{\langle B\rangle}{B},
$$

and the dose $D_{S}$ at a location on the surface, in line with a high beta straight section of length $\ell(\mathrm{m})$, is

$$
D_{S} \approx 6.7 \times 10^{-24} \frac{I_{\mu} E^{3} t}{d} \ell\langle B\rangle \text {. }
$$

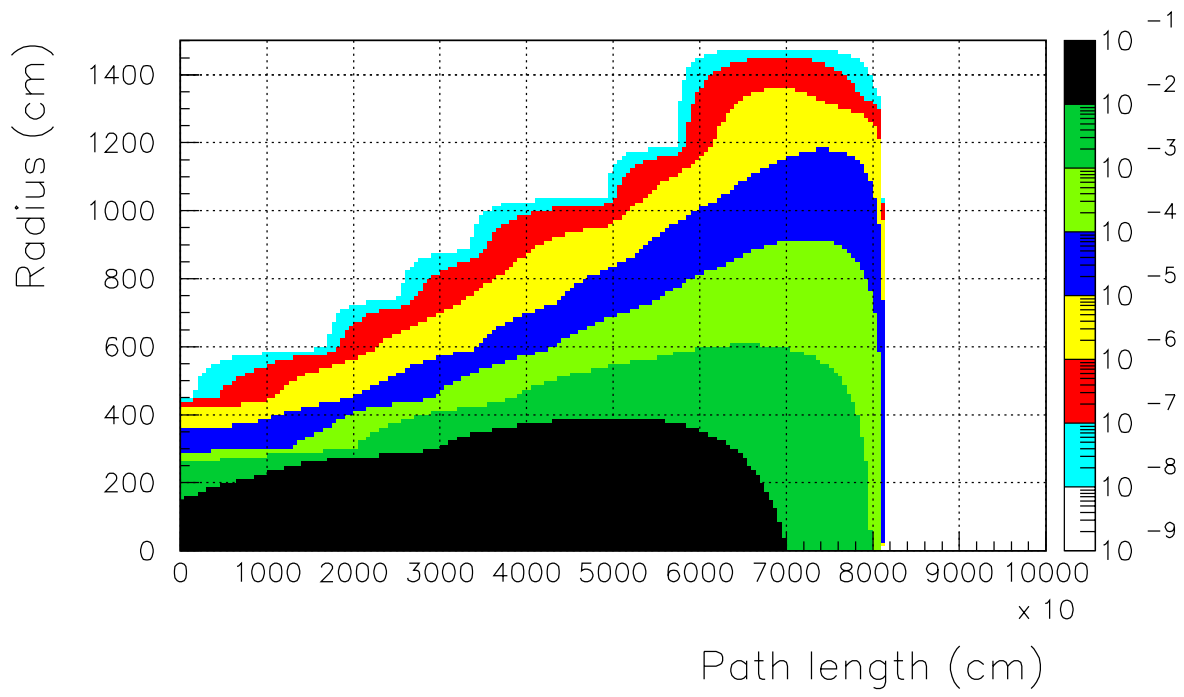

FIG. 61. (Color) Isodose contours in the soil/rock $\left(\rho=2.24 \mathrm{~g} / \mathrm{cm}^{3}\right)$ for $250 \mathrm{GeV}$ muons extracted at $3 \times 10^{13}$ per second. Righthand scale is dose rate in rem/s. 


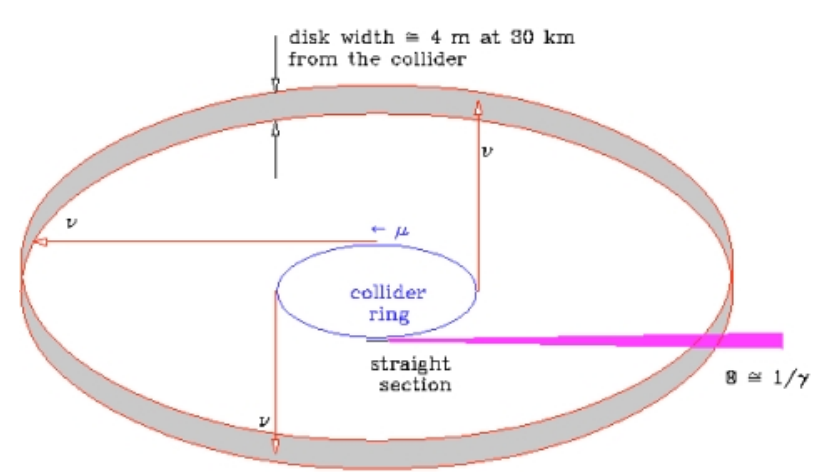

FIG. 62. (Color) Neutrino radiation disk. For a $3 \mathrm{TeV}$ COM collider the neutrino radiation width is $\approx 4 \mathrm{~cm}$ at a distance of $30 \mathrm{~km}$. A hot spot produced by a $0.1 \mathrm{~m}$ straight section in the ring contains roughly twice the number of neutrinos on the disk on average, depending on the details of the collider lattice.

The equation for $D_{S}$ assumes that the average divergence angles satisfy the condition $\sigma_{\theta} \ll \frac{1}{\gamma}$. This condition is not satisfied in the straight sections approaching the IP, and these regions, despite their length, do not contribute a significant dose.

For the $3 \mathrm{TeV}$ parameters given in Table $\mathrm{I}$ and muon currents $I_{\mu}=6 \times 10^{20} \mu^{-} / \mathrm{yr},\langle B\rangle=6 \mathrm{~T}, B=$ $10 \mathrm{~T}$, and depth $=500 \mathrm{~m}$, and taking the federal limit on off site radiation dose/yr, $D_{\text {fed }}$, to be $1 \mathrm{~m} \mathrm{~Sv} / \mathrm{yr}$ (100 mrem/yr), the annual dose $D_{B}$ (one year is defined as $10^{7} \mathrm{~s}$ ) in the plane of a bending dipole is

$$
D_{B}=1.07 \times 10^{-5} \mathrm{~Sv} \approx 1 \% D_{\mathrm{fed}},
$$

and for a straight section of length $0.6 \mathrm{~m}$ it is

$$
D_{S}=9.7 \times 10^{-5} \mathrm{~Sv} \approx 10 \% D_{\mathrm{fed}},
$$

which may be taken to be within a reasonable limit. The general trend of these expressions has been verified by Monte Carlo simulations [221] using MARS. In particular, for the $3 \mathrm{TeV}$ case the needed depth to stay within $1 \% D_{\text {fed }}$ is $300 \mathrm{~m}$ instead of $500 \mathrm{~m}$.

Special care will be required in the lattice design to assure that no field-free region longer than $0.6 \mathrm{~m}$ is present. This may sound difficult, but it may be noted that the presence of a field of even $1 \mathrm{~T}$ is enough to reduce the dose to a level below the federal limit. The application of such a field over all $\mathrm{rf}$ and other components seems possible [221].

For lower energy machines, the requirements rapidly get easier: a $0.5 \mathrm{TeV}$ machine at $100 \mathrm{~m}$ depth could have $25 \mathrm{~m}$ long sections, for the same surface dose. For a $100 \mathrm{GeV}$ machine the doses are negligible.

For machines above $3 \mathrm{TeV}$, various strategies can be employed:

(i) The machines could be built at greater depths (mines many $\mathrm{km}$ deep are common). (ii) The vertical beam orbits in the machine could be varied so as to spread the plane of radiation and thus reduce the peak doses.

(iii) The specific locations in line with straight sections could be purchased and restricted.

(iv) Straight sections could be shortened further by using continuous combined function magnets.

(v) The machines could be built on an island, but this could have difficulties associated with access to power and other utilities.

But for any large increase in energy, to $10 \mathrm{TeV}$ for instance, some reduction in muon beam flux probably will be required. The resultant loss of luminosity might be made up in a number of ways [4]:

(i) The beam-beam tune shift constraint could be avoided by introducing a conducting medium (e.g., liquid lithium) at the interaction point [222].

(ii) The focusing strength could be increased by the use of plasma or another exotic focusing method.

(iii) Better cooling could be developed. Optical stochastic cooling [139], for instance, might reduce the emittances by many orders of magnitude, thus greatly reducing the required beam currents. Indeed, such cooling would require lower currents to function appropriately.

Such options will need future study.

\section{Muon decay background}

With $4 \times 10^{12}$ muons per bunch in a $2+2 \mathrm{TeV}$ collider ring there are approximately $4 \times 10^{5}$ muon decays per meter giving rise to high energy electrons. These offenergy, off axis electrons undergo bremsstrahlung when they traverse magnetic fields. When they exit the beam pipe they interact and produce electromagnetic showers and, to a lesser extent, hadrons and muons. Much of this debris can be locally shielded, so the primary concern is muon decays near the interaction point [44]. This is the background we discuss in some detail below.

Detailed Monte Carlo simulations of electromagnetic, hadronic, and muon components of the background $[44,195,206,211,221,223,224]$ have been performed using the MARS [115] and GEANT [151] codes. The most recent study [223] has been done with GEANT. Figure 63 shows the final $130 \mathrm{~m}$ of the $2+2 \mathrm{TeV}$ detector region in this study. It includes the final four quadrupoles, dipoles, and a solenoidal field surrounding the detector. This study (i) followed shower neutrons and photons down to $40 \mathrm{keV}$ and electrons to $25 \mathrm{keV}$, and (ii) used a tungsten shield over the beam, extending outward to an angle of $20^{\circ}$ from the axis. (iii) Inside this shield, the clear radius has a minimum, in the high energy cases, at a distance from the IP of $1.1 \mathrm{~m}(80 \mathrm{~cm}$ for $50+50 \mathrm{GeV})$. At this point, and in an expanding cone beyond it, the clear radius is maintained at approximately $4 \sigma$ of the beam size. (iv) Between this minimum aperture point and the IP, the clear radius follows an inverse cone, increasing as 


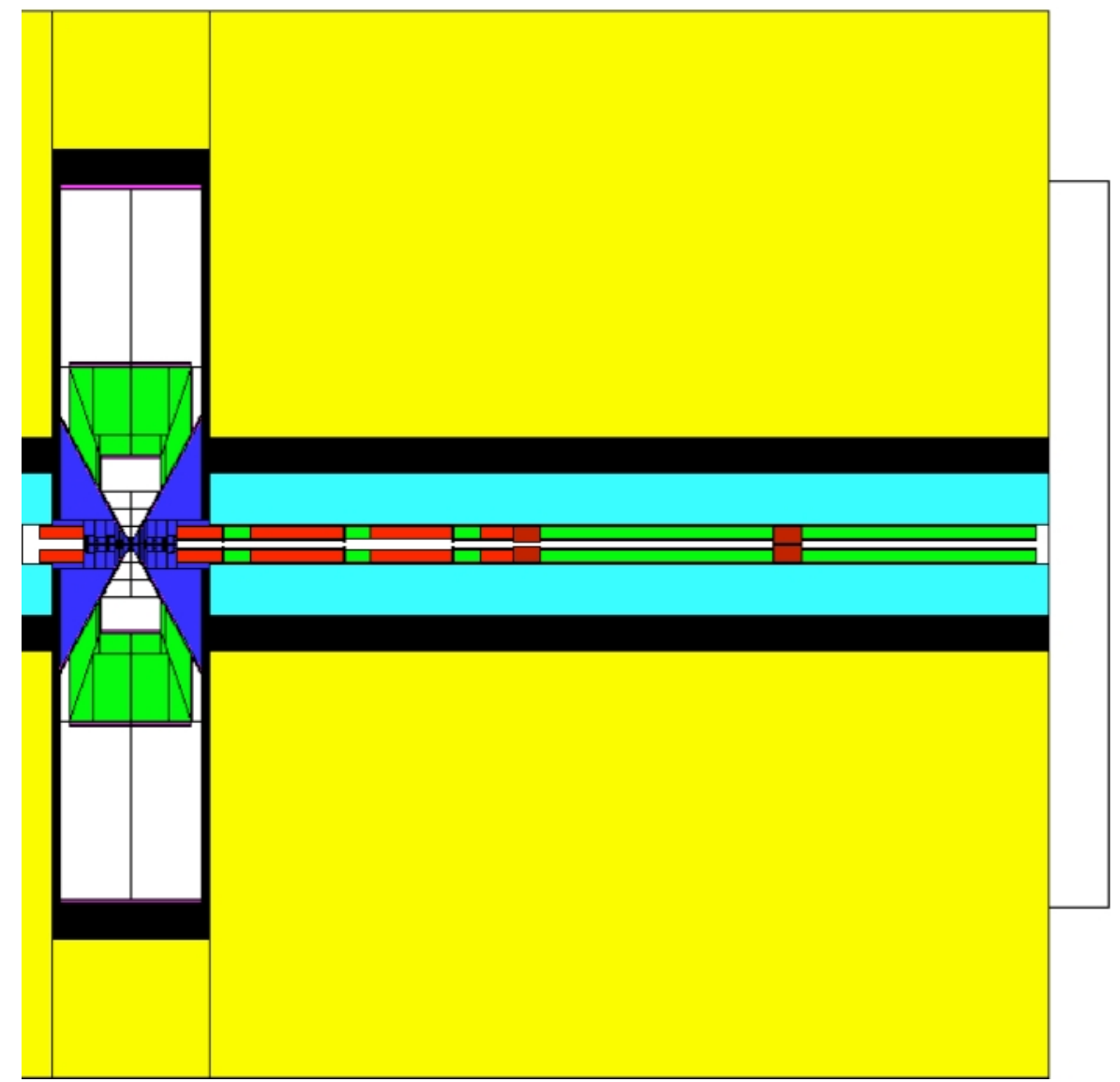

FIG. 63. (Color) Region up to $130 \mathrm{~m}$ from the IP, of the $2+2 \mathrm{TeV}$ interaction region modeled in GEANT. The triangular blue regions represent tungsten shielding. On the right-hand side of the figure, the red areas represent quadrupoles in the beam line. The areas around the IP represent the various detector volumes used in the calculations of particle fluences. The detector (white and green areas) is $10 \mathrm{~m}$ in diameter and $20 \mathrm{~m}$ long.

it approaches the IP, with an angle a little greater than the $4 \sigma$ of the beam divergence. These cones are designed so that the detector could not "see" any surface directly illuminated by the initial decay electrons, whether in the forward or backward (albedo) direction (see Fig. 64). (v) The resulting open space between the IP and the tip of the cone is approximately $3 \mathrm{~cm}$ in the $4 \mathrm{TeV}$ and $500 \mathrm{GeV} \mathrm{COM}$ cases, and approximately $6 \mathrm{~cm}$ in the $100 \mathrm{GeV}$ COM case. (vi) The inner surface of each shield is shaped into a series of collimating steps and slopes to maximize the absorption of electron showers from electrons at very small angles to the cone surface, thus reducing the funneling of low energy electrons down the pipes. (vii) Further upstream, prior to the first quadrupole (from 2.5 to $4 \mathrm{~m}$ in the Higgs case), an $8 \mathrm{~T}$ dipole, with collimators inside, is used to sweep decay electrons before the final collimation.

Note that there is currently an inconsistency, in the very low $\Delta p / p$ Higgs factory case, between the short open space between shields $( \pm 6 \mathrm{~cm})$ and the rms source length $\left(\sigma_{\text {source }}=1 / \sqrt{2} \sigma_{z}\right)$ of $10 \mathrm{~cm}$. Some modifications to the parameters and shielding design will be required for this case.
Every modern detector will have to be able to identify and reconstruct secondary vertices such as those associated with $b$-quark decays. In order to estimate the viability of a vertex detector we have to show that the occupancy of its elements is not higher than about $1 \%$. Figure 65 shows the occupancy as a function of radial distance from the interaction point for the three COM energies studied: $0.1,0.5$, and $4 \mathrm{TeV}$. The occupancy was calculated for silicon pads of $300 \mu \mathrm{m} \times 300 \mu \mathrm{m}$, and assuming interaction probabilities of 0.003 and 0.0003 for low energy photons and neutrons, respectively. One can observe that the total occupancy (left-hand figure) is above $1 \%$ for small radii. Most of the hits are due to conversions of photons. The occupancy due to hits resulting from charged particles is below 1\% (right-hand figure). One can lower the occupancy at small radii by using smaller pixel sizes, as indicated in Table $\mathrm{XV}$, as well as by using innovative detector ideas as described in the next section.

Table XV gives the hit density for the Higgs factory from the various sources and the occupancy of pixels of the given sizes; in each case the number is given per bunch crossing. The hit density for the higher energy 


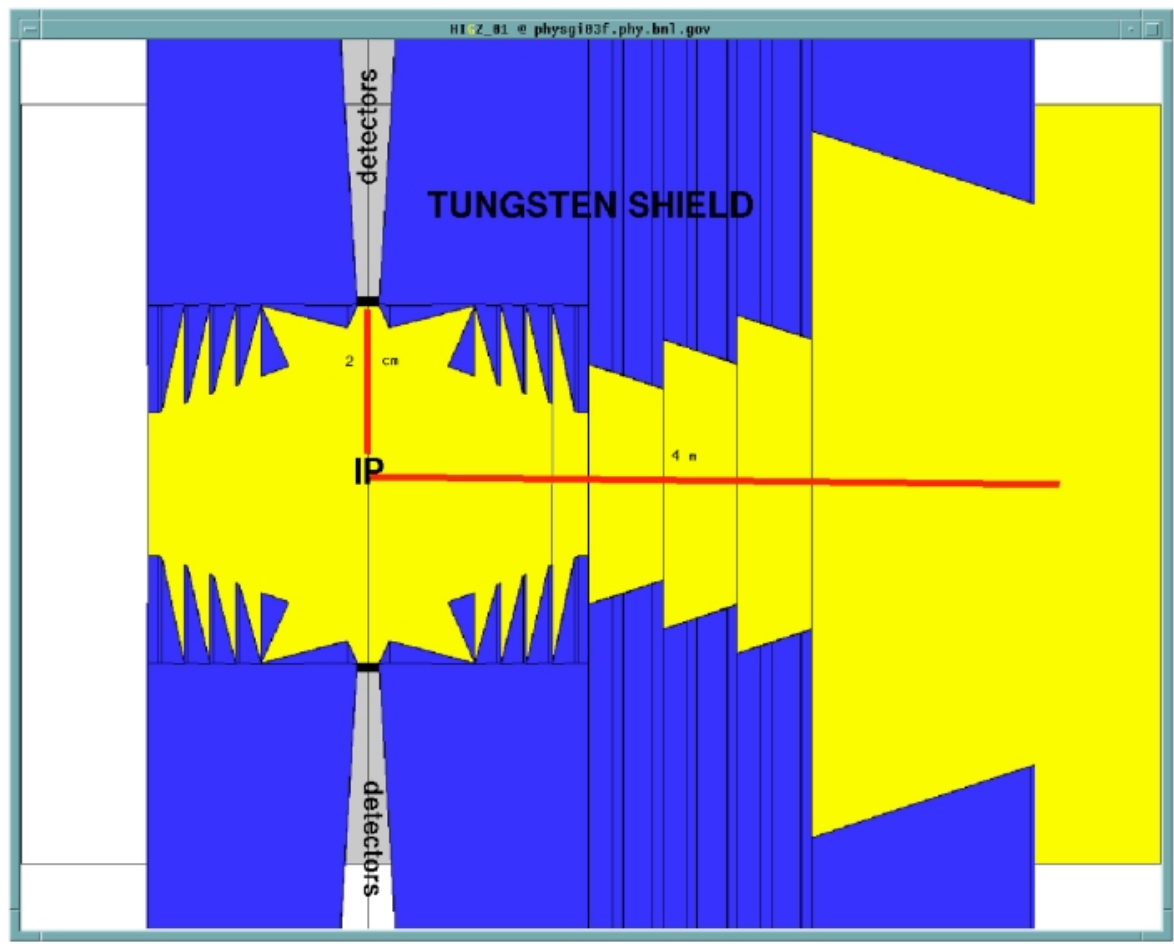

FIG. 64. (Color) Detail of the tungsten shielding designed for the $50+50 \mathrm{GeV}$ case. It is designed so that the detector is not connected by a straight line with any surface hit by decay electrons in forward or backward directions. The picture extends out to a radius of $6 \mathrm{~cm}$ and, on the right, to a distance $4 \mathrm{~m}$ from the IP. The dipole from $2.5-4.0 \mathrm{~m}$ is not shown.

machines is found to be somewhat lower due to the smaller decay angles of the electrons.

The radiation damage by the neutrons on a silicon detector has also been estimated. In the Higgs case, at $5 \mathrm{~cm}$ from the vertex, the number of hits from neutrons above $100 \mathrm{keV}$ is found to be $1.8 \times 10^{13} / \mathrm{cm}^{2}$ per year. This is significantly less than that expected at the LHC which is now ordering silicon detectors claimed to survive $5 \times 10^{14}$ hits, approximately 3 times that assumed here. The damage for silicon detectors in the higher energy machines is of the same order (see Table XVI).



\section{Halo background}

Muon halo refers to those muons which are lost from the beam bunch as it circulates around the collider ring. In conventional electron or proton accelerators, beam particles which are lost away from the IP are of little concern as they can be locally shielded. However, muons can traverse long distances and therefore have the potential to generate background in a detector. The magnitude of this background depends on a detailed knowledge of the injected beam profile and a credible model for

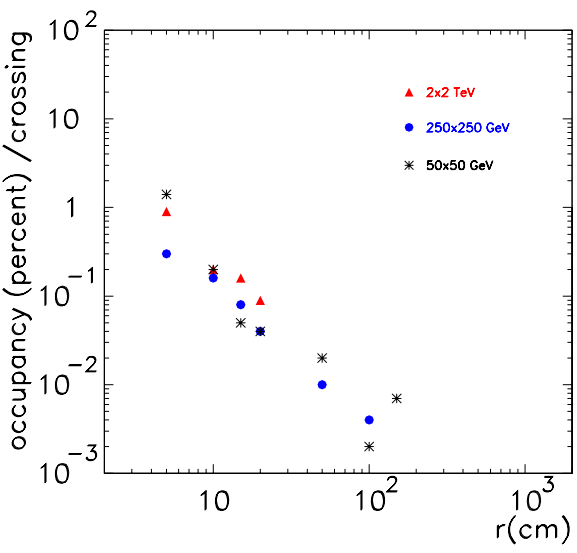

FIG. 65. (Color) Occupancy for $300 \mu \mathrm{m} \times 300 \mu \mathrm{m}$ silicon pads, as a function of the radius for the three energies studied. Lefthand figure shows the total occupancy and the right-hand figure shows the occupancy from hits resulting from charged particles. 
TABLE XV. Detector backgrounds from $\mu$ decay.

\begin{tabular}{|c|c|c|c|c|}
\hline Radius (cm) & 5 & 10 & 20 & 100 \\
\hline Photons hits $\left(\mathrm{cm}^{-2}\right)$ & 26 & 6.6 & 1.6 & 0.06 \\
\hline Neutrons hits $\left(\mathrm{cm}^{-2}\right)$ & 0.06 & 0.08 & 0.2 & 0.04 \\
\hline Charged hits $\left(\mathrm{cm}^{-2}\right)$ & 8 & 1.2 & 0.2 & 0.01 \\
\hline Total hits $\left(\mathrm{cm}^{-2}\right)$ & 34 & 8 & 2 & 0.12 \\
\hline Pixel size $(\mu \mathrm{m} \times \mu \mathrm{m})$ & $60 \times 150$ & $60 \times 150$ & $300 \times 300$ & $300 \times 300$ \\
\hline Total occupancy (\%) & 0.6 & 0.14 & 0.4 & 0.02 \\
\hline Occupancy charged (\%) & 0.14 & 0.02 & 0.04 & 0.002 \\
\hline
\end{tabular}

beam halo and beam losses. More work is needed before these are well enough understood. Nevertheless, it is clear that the beam will need careful preparation before injection into the collider, and the injection system will have to be precise and free of ripple.

The collimation system described in the previous subsection was designed to scrape the beam both initially and during the 1000 turns, to assure that all loss occurs at the scraper and not near the IP. That study indicated suppressions better than $10^{3}$ of background in the detector [196].

Beam loss must be limited as far as possible. Gas scattering has been studied [225] and shown to give a negligible contribution. The effects of beam-beam scattering are under study and need further work. Momentum spread tails from uncorrected wake field effects must be controlled. Assuming that the total loss from all causes, after injection and the first few turns, is less than $10^{-4}$ in 1000 turns (i.e., $10^{-7}$ per turn), then the number of background muons passing through the detector should be less than $800\left(2 \times 4 \times 10^{12} \times 10^{-7} \times 10^{-3}\right)$ per turn. This is a low density of tracks per $\mathrm{cm}^{2}$ and should be acceptable, but lower losses or better scraping would be desirable.

\section{E. Pair production}

Coherent beam-beam electron pair production (beamstrahlung) has been shown $[208,226]$ to be negligible, but the incoherent pair production (i.e., $\left.\mu^{+} \mu^{-} \rightarrow \mu^{+} \mu^{-} e^{+} e^{-}\right)$in the $4 \mathrm{TeV}$ collider case is significant.

The cross section is estimated to be $10 \mathrm{mb}$ [226], which would give rise to a background of $\approx 3 \times 10^{4}$ electron pairs per bunch crossing. The electrons at production do not have significant transverse momentum but the fields of the on-coming $3 \mu \mathrm{m}$ bunch can deflect them toward the detector. A simple program was written to track electrons from close to the axis (the worst case) as they are deflected away from the bunch center. Once clear of the opposing bunch, the tracks spiral under the influence of the experimental solenoid field. Figure 66 shows the radii vs length of these electron tracks for initial momenta from 3.8 to $3000 \mathrm{MeV}$ in geometric steps of $\sqrt{2}$. Figure 66(a) is for a solenoidal field of $2 \mathrm{~T}$ and Fig. 66(b) for $4 \mathrm{~T}$. In the $2 \mathrm{~T}$ case tracks with initial energy below $30 \mathrm{MeV}$ do not make it out to a detector at $10 \mathrm{~cm}$, while those above $100 \mathrm{MeV}$ have too small an initial angle and remain within the shield. Approximately 10\% (3000 tracks) of these are in this energy range and pass through a detector at $10 \mathrm{~cm}$. The track fluences at the ends of the detector are less than 10 tracks per $\mathrm{cm}^{2}$ which should not present a serious problem. At $5 \mathrm{~cm}$, there are 4500 tracks giving a fluence of 30 per $\mathrm{cm}^{2}$, which is also probably acceptable. If the detector solenoid field is raised to $4 \mathrm{~T}$, then no electrons reach $10 \mathrm{~cm}$ and the flux at $5 \mathrm{~cm}$ is reduced by a factor of 2 .

\section{F. Bethe-Heitler muons}

The GEANT/MARS studies [44,211,215] also found a significant flux of muons with quite high energies, from $\mu$ pair production in electromagnetic showers (BetheHeitler). Figures 67 and 68 show the trajectories of typical muons from their sources in the shielding around the beam pipe to the detector. Figure 67 is for a $4 \mathrm{TeV}$ COM collider, where the muons have high energy and long path lengths. A relatively long $(130 \mathrm{~m})$ section of beam pipe prior to the detector is shown. Figure 68 is for the $100 \mathrm{GeV}$ COM collider for which, since the muons have rather short path lengths, only a limited length of beam pipe is shown. Note that the scales are extremely distorted: the $20^{\circ}$ shielding cones on the right-hand side of the figures appear at steeper angles.

TABLE XVI. Radiation damage by neutrons on silicon detectors. The working assumptions are 1000 turns, $15 \mathrm{~Hz}$ and $1 \mathrm{yr}=10^{7} \mathrm{~s}$. An acceptable number of hits per year is $1.5 \times 10^{14}$.

\begin{tabular}{lcccc}
\hline \hline $\begin{array}{l}\mathrm{COM} \\
(\mathrm{TeV})\end{array}$ & $\begin{array}{c}\mu \text { 's/bunch } \\
\left(10^{12}\right)\end{array}$ & $\begin{array}{c}\text { Neutrons/ } \mathrm{cm}^{2} / \mathrm{crossing} \\
(\text { above } 100 \mathrm{keV})\end{array}$ & $\begin{array}{c}\text { Hits } / \mathrm{cm}^{2} / \mathrm{yr} \\
\left(10^{13}\right)\end{array}$ & $\begin{array}{c}\text { Lifetime } \\
(\mathrm{yr})\end{array}$ \\
\hline 4 & 2 & 100 & 3 & 5 \\
0.5 & 4 & 50 & 3 & 5 \\
0.1 & 4 & 30 & 1.8 & 8 \\
\hline \hline
\end{tabular}



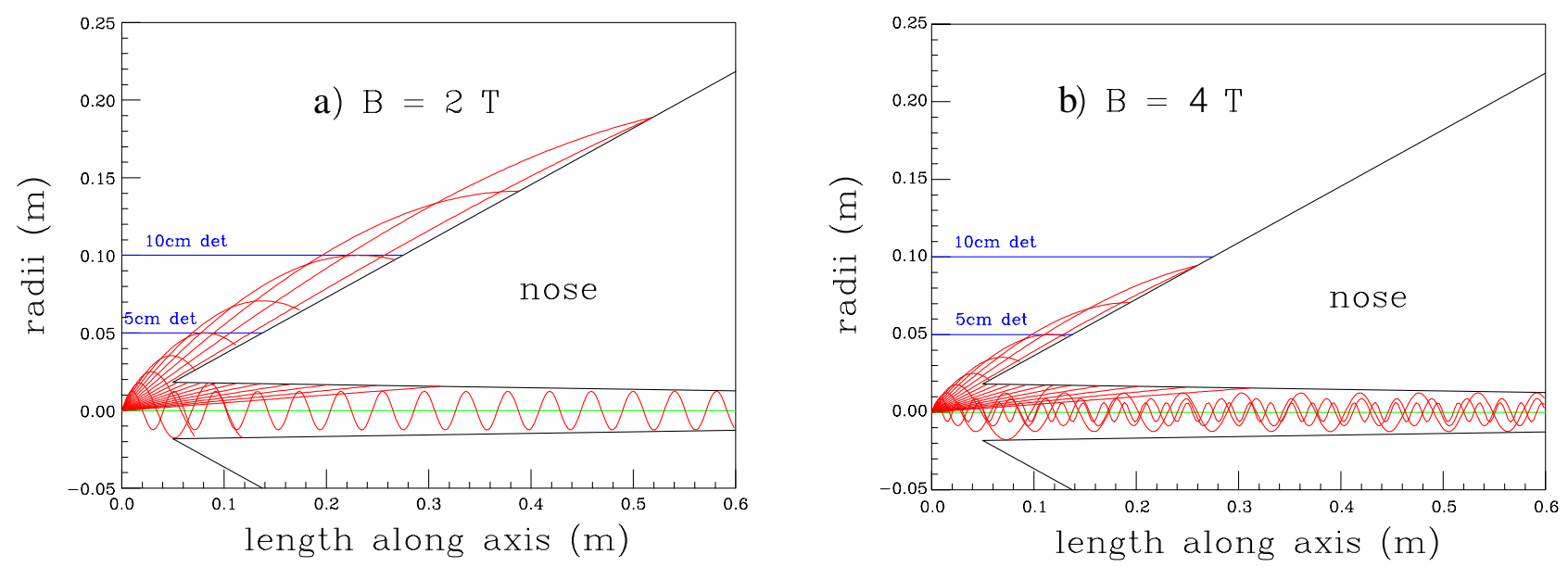

FIG. 66. (Color) Radius vs length of electron pair tracks for initial momenta from 3.8 to $3000 \mathrm{MeV}$ in geometric steps of $\sqrt{2}$ : (a) for a solenoid field of $2 \mathrm{~T}$ and (b) for $4 \mathrm{~T}$.

The most serious effect appears to arise when these muons make deeply inelastic interactions and deposit spikes of energy in the electromagnetic and hadronic calorimeters. This is not serious in the Higgs case, for which the fluxes and cross sections are low, but at the higher collider energies they generate significant fluctuations in global parameters, such as transverse energy and missing transverse energy.

Table XVII gives some parameters of the muons for three different machine energies. In the $4 \mathrm{TeV}$ and
$500 \mathrm{GeV}$ COM cases, massive lead shielding outside the focus quadrupoles has been included.

Figures 69 and 70 show energy deposition from BetheHeitler muons in a typical bunch crossing. These depositions are plotted against the cosine of the polar angle and azimuthal angle in the calorimeter for $4 \mathrm{TeV}$ and for $500 \mathrm{GeV}$ COM, respectively. The massive lead shielding referred to above was not included in this study. Righthand plots in Figs. 69 and 70 show the same distributions with a $1 \mathrm{~ns}$ timing cut. It is seen that the timing cut, if it is

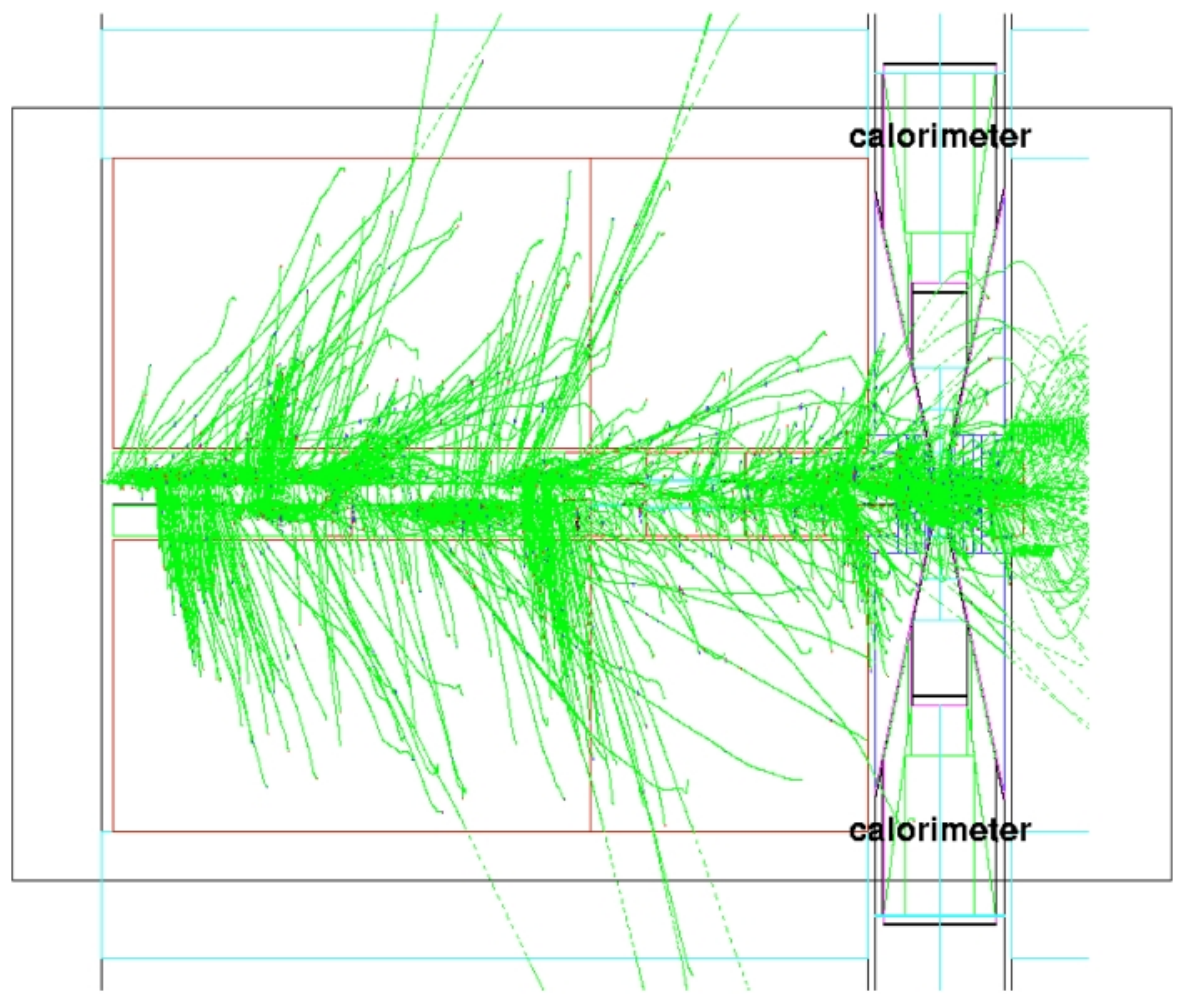

FIG. 67. (Color) Trajectories of typical Bethe-Heitler muons from their source in the shielding around the beam pipe to the detector for a $4 \mathrm{TeV} \mathrm{COM}$ collider. As indicated in the text the scales are extremely distorted, the total horizontal length is $\approx 130 \mathrm{~m}$, and the outer edge of the calorimeter is $\approx 4 \mathrm{~m}$. Notice that $<1 \%$ of the tracks end in the calorimeter (see Table XVII). 


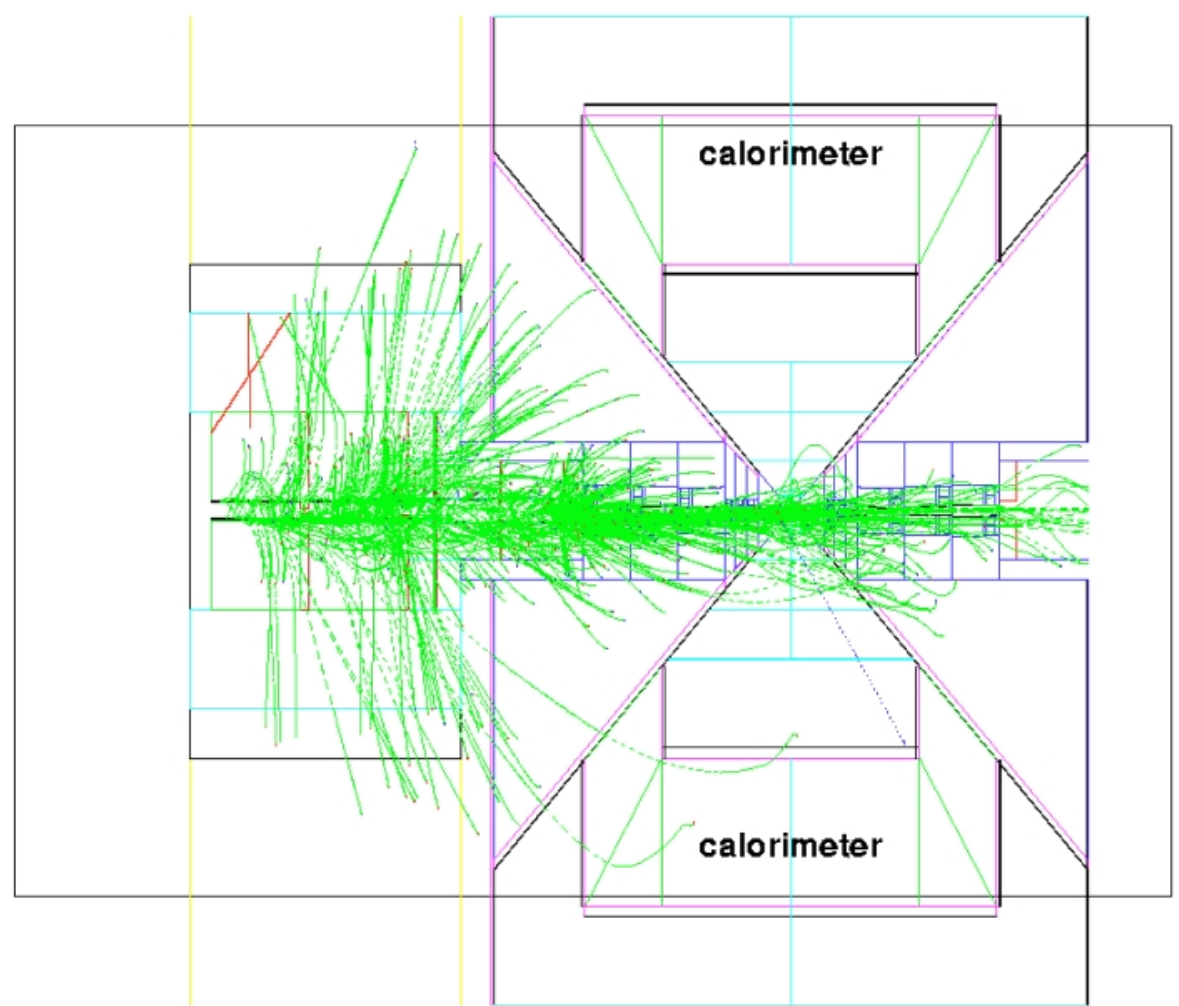

FIG. 68. (Color) Trajectories of typical Bethe-Heitler muons from their source in the shielding around the beam pipe to the detector for a $100 \mathrm{GeV} \mathrm{COM}$ collider. As indicated in the text the scale is extremely distorted, the total horizontal length is $\approx 20 \mathrm{~m}$, and the outer edge of the calorimeter is $\approx 4 \mathrm{~m}$. Notice that $<0.5 \%$ of the tracks end in the calorimeter (see Table XVII).

possible, is effective in removing energy spikes at small rapidity, but has little effect in the forward and backward directions. The overall reduction in energy deposition is about a factor of 2 .

The energy spikes can cause at least three problems: (i) they affect the triggers and event selections based on overall or transverse energy balance, (ii) they can generate false jets, and (iii) they can give errors in the energies of real jets. After a pedestal subtraction, the effects on energy balances do not seem serious. The generation of false jets can be eliminated by a longitudinal energy distribution cut without introducing significant inefficiency. Energy errors in real jets appear to be the most serious problem. They can be reduced by the application of radial energy distribution cuts, but such cuts introduce significant inefficiencies for lower energy jets. More study is needed.

Earlier studies [224] with MARS, using less sophisticated shielding, gave results qualitatively in agreement with those from GEANT.

TABLE XVII. Bethe-Heitler muons.

\begin{tabular}{lccc}
\hline \hline COM collider energy $(\mathrm{TeV})$ & 4 & 0.5 & 0.1 \\
Assumed source length $(\mathrm{m})$ & 130 & 33 & 20 \\
$\mu\left(p_{\text {muon }}>1 \mathrm{GeV} / c\right)$ per electron & $5.4 \times 10^{-4}$ & $8.3 \times 10^{-5}$ & $9.6 \times 10^{-6}$ \\
Beam $\mu$ 's per bunch & $2 \times 10^{12}$ & $2 \times 10^{12}$ & $4 \times 10^{12}$ \\
Bethe-Heitler $\mu$ 's per bunch crossing $\left(\times 10^{3}\right)$ & 28 & 17.5 & 6.1 \\
$\left\langle p_{\text {muon }}\right\rangle$ initial $(\mathrm{GeV})$ & 22 & 9.5 & 25 \\
$\mu$ 's entering calorimeter & 220 & 160 & 1.8 \\
$\left\langle p_{\text {muon }}\right\rangle(\mathrm{GeV})$ & 15.4 & 6.3 & 0.4 \\
$\left\langle E_{\text {dep }}\right\rangle(\mathrm{GeV})$ & 2.9 & 1.3 & 10 \\
Total $E_{\text {dep }}(\mathrm{GeV})$ & 640 & 210 & 1 \\
$E_{\text {dep }}$ pedestal subtracted $(\mathrm{GeV})$ & 50 & 25 & 1 \\
Fluctuation in $E_{\text {dep }}(\mathrm{GeV})$ & 15 & 15 & 0.5 \\
$E_{\text {trans }}$ pedestal subtracted $(\mathrm{GeV})$ & 40 & 15 & 0.5 \\
Fluctuation in $E_{\text {trans }}(\mathrm{GeV})$ & 8 & \\
\hline \hline
\end{tabular}




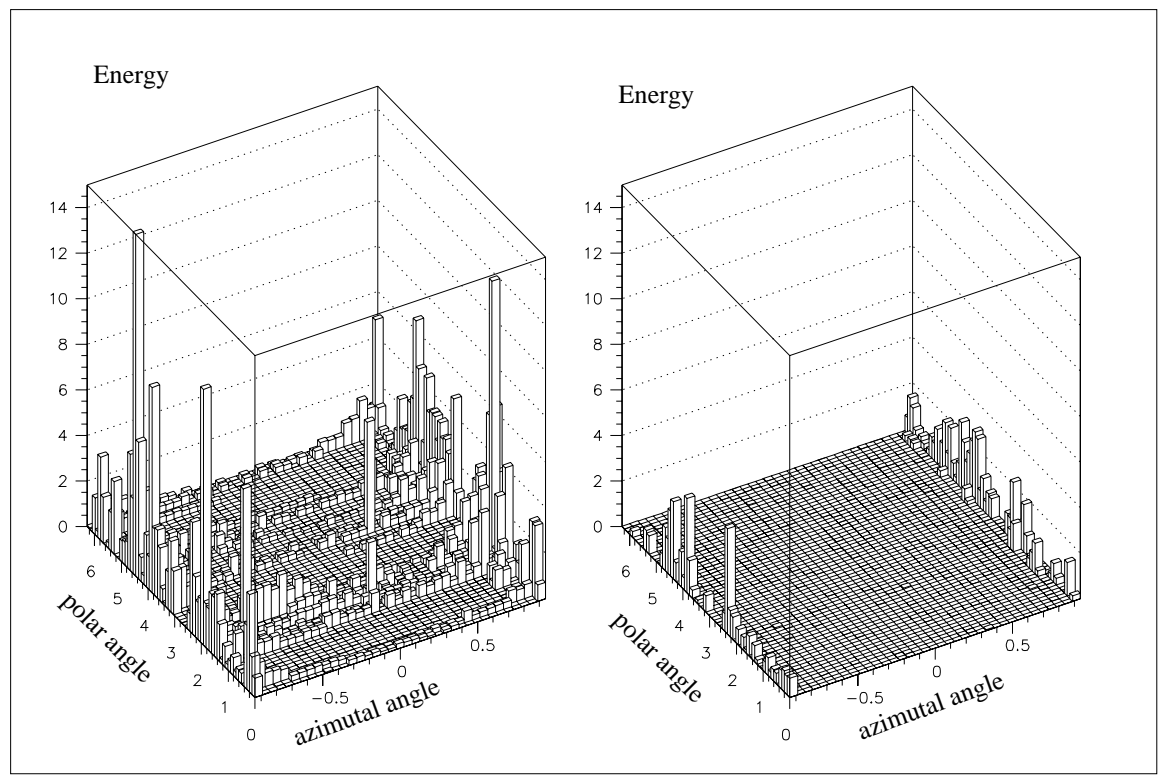

FIG. 69. The left-hand plot shows the energy deposition from Bethe-Heitler muons vs the cosine of the polar angle and azimuthal angle in the calorimeter for a $4 \mathrm{TeV} \mathrm{COM}$ collider. The right-hand plot shows the same distributions with a $1 \mathrm{~ns}$ timing cut.

\section{DETECTOR SCENARIOS}

The background consists of neutral and charged particles. For neutrons, the longitudinal and radial fluences were found to be comparable. The photons (average energy about $1 \mathrm{MeV}$ ) show a clear radial source. The charged particles and the photons do not all point back to the interaction point, but to the general vicinity of the IP, namely to the region where the $20^{\circ}$ tungsten shield becomes thinner. The flux of secondary muons (BetheHeitler pairs) is mainly longitudinal.
We would expect this background to pepper the tracking volume with random hits and produce significant energy pedestals in the calorimeter cells. These effects are considered in more detail in the following sections. In general, in designing a strawman detector that must operate in a large background flux we will want to employ as many detector channels as is practical. A strawman muon collider detector design with a few times $10^{6}$ nonpixel channels would seem reasonable [227]. Over the past few years, development of pixel detectors has resulted in a quantum jump in the number of electronic

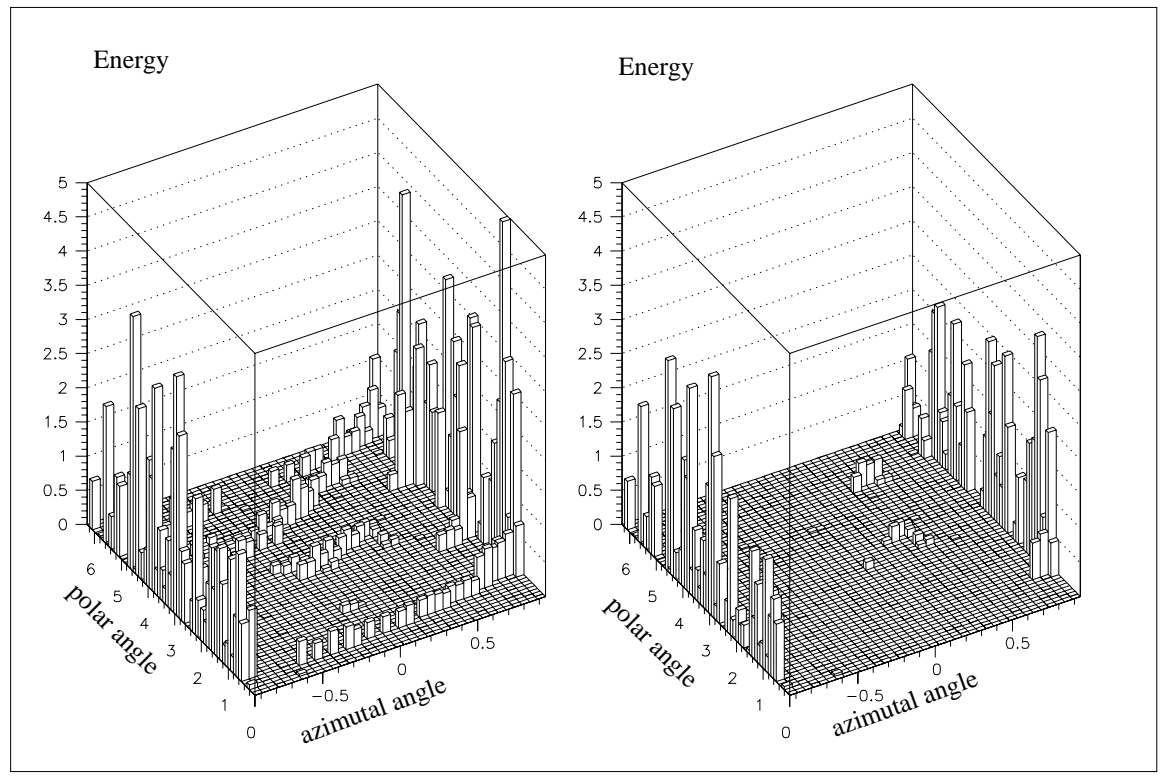

FIG. 70. The left-hand plot shows the energy deposition from Bethe-Heitler muons vs the cosine of the polar angle and azimuthal angle in the calorimeter for a $0.5 \mathrm{TeV}$ COM collider. The right-hand plot shows the same distributions with a $1 \mathrm{~ns}$ timing cut. 
channels. For example, the SLD vertex detector [228] contains $300 \times 10^{6}$ pixels, and similar numbers of pixels are planned for the LHC vertex detectors. Hence, a strawman muon collider vertex detector employing $10^{8}-10^{9}$ pixels would seem reasonable.

\section{A. Silicon vertex detector schemes}

From Table XVI, it can be seen that the radiation damage to silicon detectors is acceptable in terms of the number of hits per year and the resultant lifetime of the detector. This prompts [227] us to consider the following options for silicon vertex detector design for the muon collider:

(i) Silicon drift detector. - The idea, which is described in the muon collider feasibility study [44,33], is to exploit the time gap between bunch crossings by using the silicon drift detector technology [229] (see Fig. 71). Using $50 \times 300 \mu \mathrm{m}^{2}$ detectors it should be possible to obtain a resolution of a few microns in the drift direction. This would facilitate a very precise vertex detector, although questions of radiation hardness remain to be resolved for this option.

(ii) Columnar pixels [230]. - The idea is to exploit the very well localized primary vertex position by using long thin tracking pixels that point at the IP and therefore record large ionization signals only for tracks coming from the IP (Fig. 72). For example, one can construct $50 \times 50 \mu \mathrm{m}^{2}$ pixels that are $300 \mu \mathrm{m}$ deep. The pixels are produced using controlled feed-through-drilling technology to create a lattice of anodes and cathodes that extend through the $300 \mu \mathrm{m}$ thick wafer.

(iii) Pixel microtelescopes [231]. - The idea is to replace a single pixel layer with two layers separated by a small distance, and read them out by taking the AND between appropriate pairs. The distance between the layers is optimized so that soft $\mathrm{MeV}$ tracks (which are associated with almost $80 \%$ of the predicted background hits) produced in one layer curl up in the magnetic field before reaching the second layer. Thus, the pixel microtelescope is blind to the soft background hits and also blind to tracks that do not come from the IP. In the example shown in

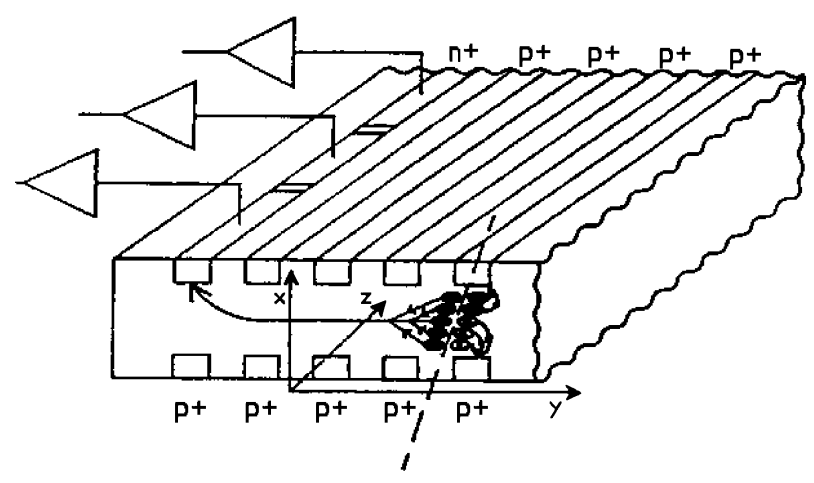

FIG. 71. (Color) Silicon drift vertex detector.

\section{Columnar Pixel Array}
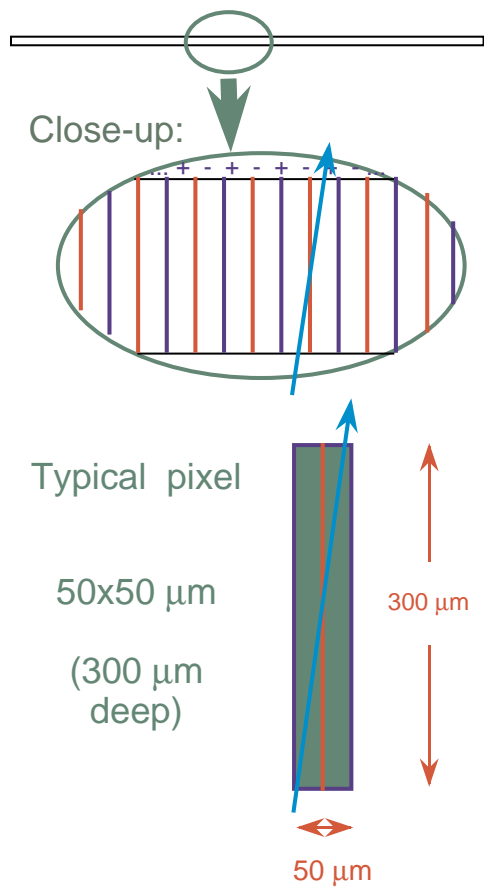

FIG. 72. (Color) Columnar pixel geometry. Courtesy of A. Sill.

Fig. 73 the top measurement layer has a finer granularity than the bottom confirmation layer. The corresponding rows in the two pixel layers can be read out with different clock speeds to maintain the correct correspondence at the

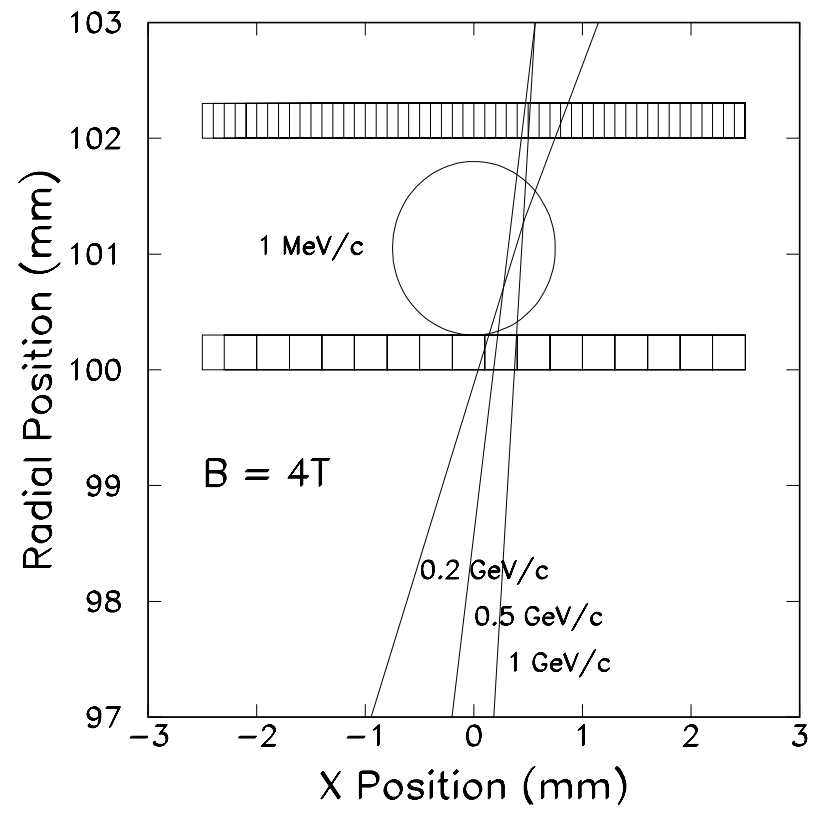

FIG. 73. Pixel microtelescope geometry 231, showing trajectories of $0.2,0.5$, and $1 \mathrm{GeV} / c$ tracks coming from the IP and bending in a $4 \mathrm{~T}$ field. 
input into the AND gate that registers valid hits in the telescope. If the readout rows are the ones parallel to the beam direction, then variable clock speeds can be used to maintain the correct accepted direction with respect to the IP.

The challenge of a high background environment is clearly fruitful ground for new ideas. The above considerations suggest that, provided silicon detectors can be used in the inner tracking volume, it should be possible to construct a vertex detector able to tag secondary vertices from short lived particles at a muon collider. Detailed simulations are currently underway to establish this more concretely.

\section{B. Outer tracking schemes}

The predicted background fluxes for a Higgs factory detector at a radius of $50 \mathrm{~cm}$ are 200 photons $/ \mathrm{cm}^{2}$, 350 neutrons $/ \mathrm{cm}^{2}$, and 0.08 charged tracks per $\mathrm{cm}^{2}$ per crossing. The neutron flux is therefore about the same as the flux in the inner tracking volume, whereas the photon and charged particle fluxes are significantly less than those predicted at smaller radii. There are two alternative tracking strategies under consideration.

(i) Low field, large tracking volume drift chamber option.-This option, which is described in the muon collider book [232], uses a TPC to exploit the $20 \mu$ s time between bunch crossings. This option is viable for the very high energy muon collider $(1.5 \times 1.5 \mathrm{TeV})$. The large neutron flux necessitates choosing a gas that does not contain hydrogen. A mixture of $90 \%$ neon plus $10 \%$ $\mathrm{CF}_{4}$ gives a drift velocity of $9.4 \mathrm{~cm} / \mu \mathrm{s}$, which is close to that required to match the bunch crossing time. High- $p_{T}$ tracks from the IP embedded in the predicted background flux have been simulated for the TPC shown in Fig. 74. The simulation includes ionization, drift and diffusion of the electrons in the gas, multiplication, and other details of the detection process. The majority of the background

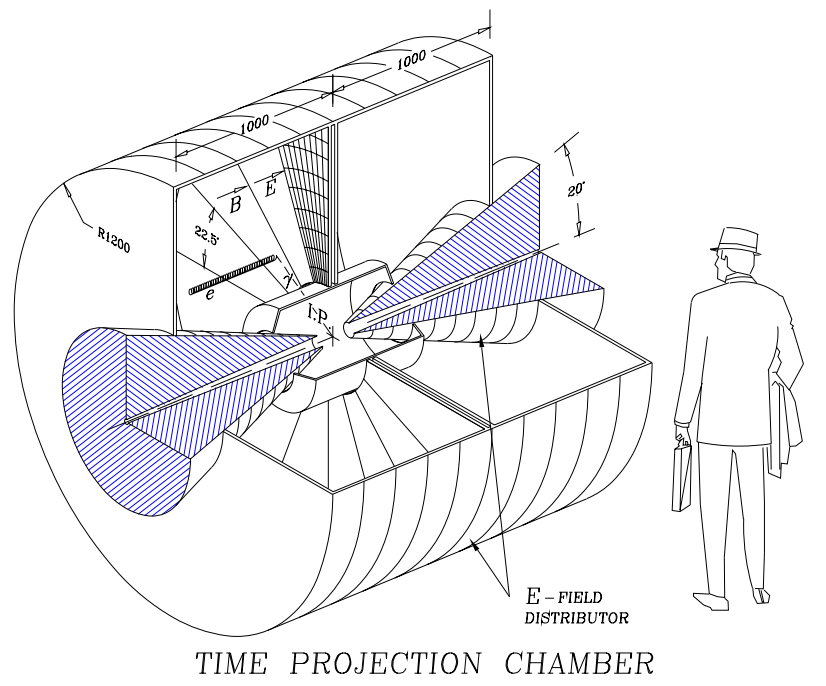

FIG. 74. (Color) Outer tracker TPC.

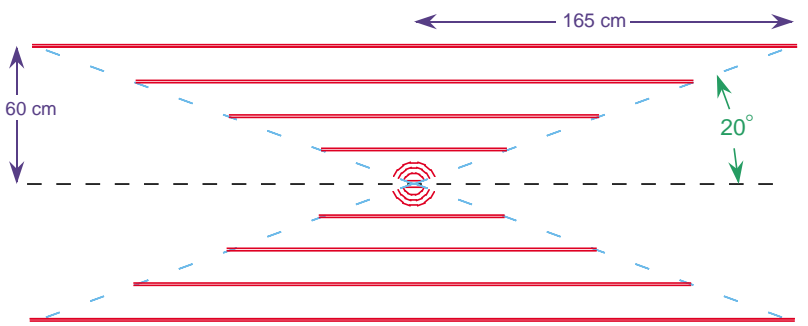

FIG. 75. (Color) Compact tracker geometry in a $4 \mathrm{~T}$ field.

hits arises from low energy Compton recoils yielding very low energy electrons that have a radius of curvature of less than $1 \mathrm{~mm}$ in the $2 \mathrm{~T}$ field. Their projection on the readout plane covers not more than one readout pitch $\left(0.3 \times 0.4 \mathrm{~cm}^{2}\right)$. These background electrons, together with the nuclear recoils from neutron scatters, yield large pulses that can be removed by cutting on the maximum acceptable pulse height. The simulation predicts that with an average background flux of 100 photons $/ \mathrm{cm}^{2}$, reasonable pulse height cuts remove only $1 \%$ of the effective TPC volume, and yield tracks of high quality. However, it was realized that positive ion buildup may be a problem with the design shown in Fig. 74. If this problem can be overcome, the design shown in the figure yields a simulated momentum resolution of about $1.2 \%$ for tracks with $p_{T}=50 \mathrm{GeV} / c$.

(ii) High field, compact silicon tracker option.-An alternative strategy is to make a compact tracker by using silicon in a high field (for example, $4 \mathrm{~T}$ ). As an example, consider the geometry shown in Fig. 75 in which a fourlayer pixel vertex detector is surrounded by a four-layer cylindrical stereo silicon microstrip detector. We take the inner layer of the vertex detector to consist of a cylinder of $50 \times 300 \mu \mathrm{m}^{2}$ pixels, and the outer three vertex layers to consist of spherical shells of $50 \times 50 \mu \mathrm{m}^{2}$ columnar pixels or pixel microtelescopes. The resolution of the microstrip detector is chosen to match that of the pixel detector. The system is assumed to correspond to $15 \%$ of a radiation length at $90^{\circ}$. Using a parametric calculation of the momentum resolution, including multiple scattering, we obtain $\sigma_{p} / p^{2}=10^{-4}\left(10^{-2}\right)(\mathrm{GeV} / c)^{-1}$ for $p=100 \mathrm{GeV} / c(1 \mathrm{GeV} / c)$.

Both the low field and high field tracking solutions look interesting and should be pursued with more complete simulations.

\section{Electromagnetic calorimeter schemes}

Background particles entering the electromagnetic calorimeter are expected to give rise to significant energy pedestals in the calorimeter cells. Consider a $4 \mathrm{~m}$ long calorimeter that is 25 radiation lengths deep, has an inner radius of $120 \mathrm{~cm}$, and is constructed from $2 \times 2 \mathrm{~cm}^{2}$ cells. This gives a total of $10^{5}$ electromagnetic calorimeter towers. The GEANT background calculation predicts 
that each cell sees on average $n_{\gamma}=4$ background photons per crossing with a mean energy $E_{\gamma}=1-2 \mathrm{MeV}$. If an electromagnetic shower occupies nine cells, then the mean background pedestal will be about $70 \mathrm{MeV}$. This pedestal can be subtracted from the measured energies. The precision of the resulting electron and photon energy measurements will depend on the fluctuations in the mean background energy per cell. For an electromagnetic shower occupying nine cells, the fluctuations in the pedestals are predicted to be about $10 \mathrm{MeV}$. This takes into account the fluctuations in the number and the energies of the background photons.

\section{Hadronic calorimeter schemes}

None of the energy generated by background photons in the electromagnetic calorimeter is expected to penetrate into the hadronic calorimeter. GEANT calculations show that the total kinetic energy deposited by neutrons in the calorimeter is of the order of $140 \mathrm{TeV}$ with an average energy of $30 \mathrm{MeV}$ per neutron per crossing for the $4 \mathrm{TeV} \mathrm{COM}$ energy case. In order to estimate what fraction of the kinetic energy of the neutrons will be visible, we should consider the materials involved. For this simulation we have presumed an equal mix by volume of liquid argon (as active medium) and copper (as absorber). At $30 \mathrm{MeV}$ we expect only a small fraction of the neutrons to knock off protons and only about $10 \%$ of the proton ionization to be visible in the liquid. Presuming a hadronic calorimeter with $10^{4}$ towers, with the material composition described above, the average energy read in the liquid argon will be of the order of $10 \mathrm{MeV}$ per tower with a fluctuation of $5 \mathrm{MeV}$. In summary, in a $50 \times 50 \mathrm{GeV}$ collider with $4 \times 10^{12}$ muons per bunch, the photons and neutrons are expected to generate pedestals of 800 and $100 \mathrm{GeV}$, respectively. The estimates for pedestal fluctuations are at or below the level of the expected electronic noise. Therefore we believe that the subtraction of these pedestals would present little problem for both the electromagnetic and the hadronic calorimeters. The presence of the high neutron background should be taken into account in choosing materials for calorimetry. Liquid argon seems a natural choice for the electromagnetic calorimeter.

The energy deposited by the Bethe-Heitler muons in the calorimeter is given in Table XVII as a function of the center-of-mass energy of the collider. For low center-of-mass energies, such as the Higgs factory, the Bethe-Heitler muons are not a problem, since there are fewer of them and they leave less energy by catastrophic bremsstrahlung in the calorimeter. For the higher energy option (4 $\mathrm{TeV}$ in the COM or higher), one should explore ways to correct for the energy deposition in the calorimeter, such as pattern recognition of the muon tracks or by using timing information.

\section{E. Muon detector schemes}

The predicted background flux is expected to be relatively modest beyond a radius of $3 \mathrm{~m}$ in the vicinity of the muon detector. Several possible technologies for muon detectors at a muon collider were discussed during Snowmass [33].

(i) Cathode strip chambers. - The idea, which is described in the muon collider book [232], is to use multiwire proportional chambers with segmented cathodes and a short (35 ns) drift time to provide prompt signals for triggering. The precision of the coordinate measurements would be expected to be of order $50 \mu \mathrm{m} \times$ a few $\mathrm{mm}$.

(ii) Threshold Cherenkov counter. - The idea is to use a gas Cherenkov radiator to exploit the directionality of Cherenkov radiation in order to select high- $p_{T}$ muons coming from the IP. The device would also give excellent timing resolution (of order $2 \mathrm{~ns}$ ).

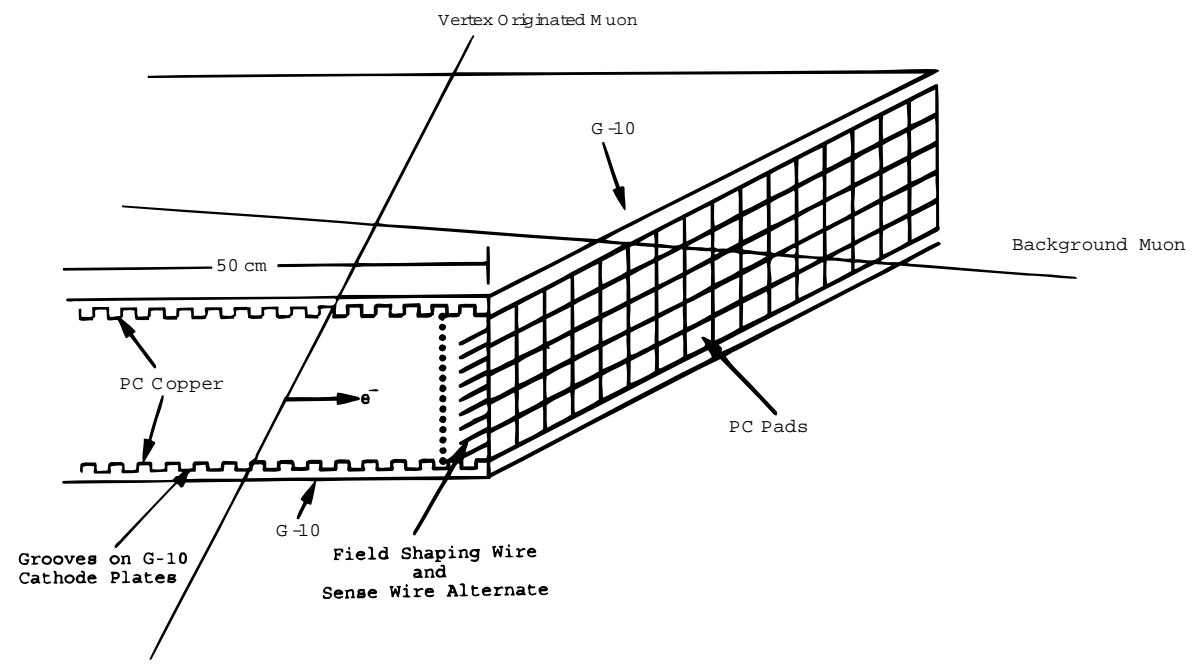

FIG. 76. Long drift jet chamber with pad readout for muon detection at a muon collider. 
(iii) Long drift jet chamber with pad readout [233] (Fig. 76). - Drift time provides the $z$ coordinate, and pad readout provides the $r-\phi$ coordinates. Directionality at the trigger level is provided by the pattern of pad hits within a limited time window. The drift field is provided by cathode strips on grooved G-10 plates. Using $90 \%$ argon plus $10 \% \mathrm{CF}_{4}$ and a maximum drift distance of $50 \mathrm{~cm}$, the maximum drift time is $5 \mu \mathrm{s}$.

At high energy in the COM, the channel $\mu^{+} \mu^{-} \rightarrow$ $\mu^{+} \mu^{-}+$Higgs boson becomes particularly attractive to study using the muon collider, if the forward going muons from the interaction can be detected [234]. The method provides a capability to search for any missing neutral state such as the Higgs boson via the missing mass technique. We are investigating methods to improve our forward muon detection capability.

\section{CONCLUSIONS}

Unlike protons, muons are pointlike, but, unlike electrons, they emit relatively little synchrotron radiation and therefore can be accelerated and collided in rings.

Another advantage resulting from the low synchrotron radiation is the lack of beamstrahlung and the possibility of very small collision energy spreads. A beam energy spread of $\Delta E / E$ of $0.003 \%$ is considered feasible for a $100 \mathrm{GeV}$ machine. It has been shown that by observing spin precession, the absolute energy could be determined to a small fraction of this width. These features become important in conjunction with the large $s$-channel Higgs production $\left(\mu^{+} \mu^{-} \rightarrow h, 43000\right.$ times larger than for $e^{+} e^{-} \rightarrow h$ ), allowing precision measurements of the Higgs mass, width, and branching ratios. A higher energy muon collider can also distinguish the nearly degenerate heavy Higgs bosons $H^{0}$ and $A^{0}$ of the minimal supersymmetric extension of the standard model, since these states can also be produced in the $s$ channel. We have also examined the ability of the muon collider to study techniresonances, do a high luminosity study of $Z$ boson physics, and scan the $W$ and $t \bar{t}$ thresholds to make precision mass measurements as well as SUSY and strongly interacting $W$ boson physics. The high luminosity proton driver and the cold low energy muons permit the study of rare kaon and muon decays. Muon storage rings will permit low-systematics studies of neutrino oscillations for a wide range of mixing angle and $\delta m^{2}$ phase space with hitherto unattainable sensitivity.

Such machines are clearly desirable. The issues are (i) whether they can be built and physics done with them and (ii) what they will cost.

Much progress has been made in addressing the first question and the answer, so far, appears to be yes. It is too early to address the second.

We have studied machines with COM energies of 0.1 , 0.4 , and $3 \mathrm{TeV}$, defined parameters, and simulated many of their components. Most recent work has been done on the $0.1 \mathrm{TeV}$ first muon collider, the energy taken to be representative of the actual mass of a Higgs particle. A summary of progress and challenges follows:

(a) Proton driver. - The specification of the proton driver for the three machines is assumed the same: $10^{14}$ protons/pulse at an energy above $16 \mathrm{GeV}$ and 1-2 ns rms bunch lengths. There have been three studies of how to achieve these parameters. The most conservative, at $30 \mathrm{GeV}$, is a generic design. Upgrades of the FNAL (at $16 \mathrm{GeV}$ ) and BNL (at $24 \mathrm{GeV}$ ) accelerators have also been studied. Despite the very short bunch requirement, each study has concluded that the specification is attainable. Experiments are planned to confirm some aspects of these designs.

(b) Pion production and capture. - Pion production has been taken from the best models available, but an experiment (BNL-E910) that has taken data, and is being analyzed, will refine these models. The assumed $20 \mathrm{~T}$ capture solenoid will require state of the art technology. Capture, decay, and phase rotation have been simulated, and have achieved the specified production of 0.3 muons per initial proton. The most serious remaining issues for this part of the machine are (i) the nature and material of the target: The baseline assumption is that a liquid metal jet will be used, but the effects of shock heating by the beam, and of the eddy currents induced in the liquid as it enters the solenoid, are not yet fully understood. (ii) The maximum rf field in the phase rotation: For the short pulses used, the current assumptions would be reasonably conservative under normal operating conditions, but the effects of the massive radiation from the nearby target are not known.

Both these questions can be answered in a target experiment planned to start within the next two years at the BNL AGS.

Polarization of the muon beams represents a significant physics advantage and is an important feature of a muon collider. Polarized muon beams are possible. Muons are produced with $100 \%$ polarization in the rest frame of the pion, but they travel in all directions. By accepting the forward going muons, it is easy to obtain $25 \%$ polarization in either beam easily. The amount of polarization can be increased with an accompanying price in luminosity.

(c) Cooling. - The required ionization cooling is the most difficult and least understood element in any of the muon colliders studied. Ionization cooling is a phenomenon that occurs whenever there is energy loss in a strong focusing environment.

Achieving the nearly $10^{6}$ reduction required is a challenge. Cooling over a wide range has been simulated using lithium lenses and ideal (linear matrix) matching and acceleration. Examples of limited sections of solenoid lattices with realistic accelerating fields have now been simulated, but the specification and simulation of a complete system has not yet been done. Much theoretical work remains: space charge and wake fields must be included; 
lattices at the start and end of the cooling sequences must be designed; lattices including liquid lithium lenses must be studied, and the sections must be matched together and simulated as a full sequence. The tools for this work are nearly ready, and this project should be completed within two years.

Technically, one of the most challenging aspects of the cooling system appears to be high gradient rf (e.g., $36 \mathrm{MV} / \mathrm{m}$ at $805 \mathrm{MHz})$ operating in strong (5-10 T) magnetic field, with beryllium foils between the cavities.

An experiment is planned that will test such a cavity, in the required fields, in about two years time. On an approximately six year time scale, a Cooling Test Facility is being proposed that could test $10 \mathrm{~m}$ lengths of different cooling systems. If they are required, then an urgent need is to develop lithium lenses (e.g., $2 \mathrm{~cm}$ diameter, $70 \mathrm{~cm}$ long, liquid lithium lenses with $10 \mathrm{~T}$ surface fields and a repetition rate of $15 \mathrm{~Hz}$ ).

The use of $31 \mathrm{~T}$ solenoids could avoid their need, at least in the low energy first muon collider, which would ease the urgency of this rather long-term R\&D, but both options would require long-term R\&D. Meanwhile a short lithium lens is under construction at BINP.

(d) Acceleration. - The acceleration system is probably the least controversial, although possibly the most expensive, part of a muon collider. Preliminary parameters have been specified for acceleration sequences for a $100 \mathrm{GeV}$ and a $3 \mathrm{TeV}$ machine, but they need refinement. In the low energy case, a linac is followed by three recirculating or FFAG accelerators. In the high energy accelerator, the recirculating or FFAG accelerators are followed by three fast ramping synchrotrons employing alternating pulsed and superconducting magnets. The parameters do not appear to be extreme, and it does not appear as if serious problems are likely.

(e) Collider.-The collider lattices are challenging because of the requirement of very low beta functions at the interaction point, high single bunch intensities, and short bunch lengths. However, the fact that all muons will decay after about 800 turns means that slowly developing instabilities are not a problem. Feasibility lattices have been generated for a $4 \mathrm{TeV}$ case, and more detailed designs for $100 \mathrm{GeV}$ machines are being studied. In the latter case, but still without errors, $5 \sigma$ acceptances in both transverse and longitudinal phase space have been achieved in tracking studies. Beam scraping schemes have been designed for both the low energy (collimators) and high energy (septum extractors) cases.

The short bunch length and longitudinal stability problems are avoided if the rings, as specified, are sufficiently isochronous, but some $\mathrm{rf}$ is needed to remove the impedance generated momentum spread. Transverse instabilities (beam breakup) should be controlled by rf BNS damping.

The heating of collider ring superconducting magnets by electrons from muon decay can be controlled by thick tungsten shields, and this technique also shields the space surrounding the magnets from the induced radioactivity on the inside of the shield wall. A conceptual design of magnets for the low energy machine has been defined.

Although much work is yet to be done (inclusion of errors, higher order correction, magnet design, rf design, etc.), the collider ring does not appear likely to present a serious problem.

(f) Neutrino radiation and detector background.Neutrino radiation, which rises as the cube of the energy, is not serious for machines with center-of-mass energies below about $1.5 \mathrm{TeV}$. It is thus not significant for the first muon collider; but above $2 \mathrm{TeV}$, it sets a constraint on the muon current and makes it harder to achieve desired luminosities. However, advances in cooling and correction of tune shifts may still allow a machine at $10 \mathrm{TeV}$ with substantial luminosity $\left(>10^{35} \mathrm{~cm}^{-2} \mathrm{~s}^{-1}\right)$.

Background in the detector was at first expected to be a very serious problem, but after much work, shielding systems have evolved that limit most charged hadron, electron, gamma, and neutron backgrounds to levels that are acceptable. Muon background, in the higher energy machines, is a special problem that can cause serious fluctuations in calorimeter measurements. It has been shown that fast timing and segmentation can help suppress this background, and preliminary studies of its effects on a physics experiment are encouraging. The studies are ongoing.

(g) Detector scenarios. - We have considered several options for the experimental detector components for various COM energy colliders. Much work needs to be done to optimize the physics reach at each energy by feeding back the results of detailed simulations of backgrounds and signal to the detector design. Only then will the feasibility of doing physics with a muon collider be fully explored.

\section{ACKNOWLEDGMENTS}

This research was supported by the U.S. Department of Energy under Contracts No. DE-ACO298CH10886, No. DE-AC02-76CH03000, and No. DEAC03-76SF00098.

[1] M. Tigner, in Advanced Accelerator Concepts, edited by J. S. Wurtele, AIP Conf. Proc. No. 279 (AIP, New York, 1993), p. 1.

[2] G. I. Budker, in Proceedings of the 7th International Conference on High Energy Accelerators, Yerevan, 1969 (Academy of Sciences of Armenia, Yerevan, 1970), p. 33; extract in Physics Potential and Development of $\mu^{+} \mu^{-}$Colliders: Second Workshop, edited by D. Cline, AIP Conf. Proc. 352 (AIP, New York, 1996), p. 4. 
[3] G.I. Budker, in International High Energy Conference, Kiev, 1970 (unpublished); extract in Physics Potential and Development of $\mu^{+} \mu^{-}$Colliders: Second Workshop (Ref. [2]).

[4] A. N. Skrinsky, in Physics Potential and Development of $\mu \mu$ Colliders, edited by D. B. Cline, AIP Conf. Proc. No. 441 (AIP, New York, 1998), p. 249.

[5] B. J. King, BNL Report No. BNL-CAP-223-MUON-98C; in Proceedings of the European Particle Accelerator Conference, Stockholm, Sweden, 1998 (to be published).

[6] V. Bharadwaj et al., Fermilab Report No. FermilabTM-1970, 1995, Chap. 4, Table I and Fig. 8; http:// fnalpubs.fnal.gov/archive/1996/tm/TM-1970.html

[7] M.D. Church and J.P. Marriner, Annu. Rev. Nucl. Part. Sci. 43, 253 (1993).

[8] A. A. Kolomensky, Sov. J. At. Energy 19, 1511 (1965); http://puhep1.princeton.edu/mumu/physics/kolomensky/1. html

[9] G. I. Budker, Sov. J. At. Energy 22, 438 (1967); http:// puhep1.princeton.edu/mumu/physics/budker/1.html

[10] Yu. M. Ado and V.I. Balbekov, Sov. J. At. Energy 31, 731 (1971); http://puhep1.princeton.edu/mumu/physics/ ado/1.html

[11] V. I. Balbekov, in Beam Dynamics and Technology Issues for $\mu^{+} \mu^{-}$Colliders at the 9th Advanced ICFA Beam Dynamics Workshop, edited by J. Gallardo, AIP Conf. Proc. No. 372 (AIP, New York, 1996), p. 140; ftp:// ftp.mumu.bnl.gov/pub/documents/balbekov.ps

[12] Rajendran Raja and Alvin Tollestrup, Phys. Rev. D 58, 013005 (1998); http://xxx.lanl.gov/ps/hep-ex/9801004

[13] D. Neuffer, in Advanced Accelerator Concepts, edited by Frederick E. Mills, AIP Conf. Proc. No. 156 (AIP, New York, 1987), p. 201; http://www-ppd.fnal.gov/ muscan/munotes/mc-006.pdf

[14] S. Geer, in Workshop of Physics at the First Muon Collider and Front End of Muon Collider, edited by S. Geer and R. Raja, AIP Conf. Proc. No. 435 (AIP, New York, 1998) (a brief summary).

[15] A. N. Skrinsky, in Proceedings of the International Seminar on Prospects of High-Energy Physics, Morges, 1971 (unpublished); extract in Physics Potential and Development of $\mu^{+} \mu^{-}$Colliders: Second Workshop (Ref. [2]), p. 6.

[16] G. I. Budker and A. N. Skrinsky, Sov. Phys. Usp. 21, 277 (1978).

[17] A. N. Skrinsky, in High Energy Physics, edited by Loyal Durand and Lee G. Pondrom, AIP Conf. Proc. No. 68 (AIP, New York, 1981), p. 1056.

[18] A. N. Skrinsky and V. V. Parkhomchuk, Sov. J. Part. Nucl. 12, 223 (1981).

[19] A. N. Skrinsky, Sov. Phys. Usp. 25, 639 (1982).

[20] V. V. Parkhomchuk and A. N. Skrinsky, in Proceedings of the 12th International Conference on High Energy Accelerators, edited by F. T. Cole and R. Donaldson (Fermilab, Batavia, IL, 1983), p. 485; http://www-ppd.fnal.gov/muscan/munotes/mc-003.pdf

[21] A. N. Skrinsky, in Beam Dynamics and Technology Issues for $\mu^{+} \mu^{-}$Colliders at the 9th Advanced ICFA Beam Dynamics Workshop (Ref. [11]), p. 133.

[22] A. N. Skrinsky, Nucl. Phys. B (Proc. Suppl.) 51A, 201 (1996).
[23] D. Neuffer, Fermilab Report No. FN-319, 1979; http:// www-ppd.fnal.gov/muscan/munotes/mc-001.pdf

[24] D. Neuffer, in Proceedings of the 12th International Conference on High Energy Accelerators (Ref. [20]), p. 481; Part. Accel. 14, 75 (1983); reproduced in Workshop of Physics at the First Muon Collider and Front End of Muon Collider (Ref. [2]), p. 12.

[25] D. Neuffer, in Ref. [27], p. 27.

[26] Advanced Accelerator Concepts (Ref. [1]).

[27] Proceedings of the Mini-Workshop on $\mu^{+} \mu^{-}$Colliders, Napa, CA, 1992, edited by D. B. Cline [Nucl. Instrum. Methods Phys. Res., Sect. A 350, 24 (1994)].

[28] Proceedings of the Muon Collider Workshop, edited by H. A. Thiessen (Los Alamos National Laboratory Report No. LA-UR-93-866, 1993).

[29] Advanced Accelerator Concepts: Sixth Annual Conference, edited by P. Schoessow, AIP Conf. Proc. No. 335 (AIP, New York, 1995); see R. B. Palmer, D. V. Neuffer, and J. C. Gallardo, in ibid., p. 635.

[30] Physics Potential and Development of $\mu^{+} \mu^{-}$Colliders: Second Workshop (Ref. [2]).

[31] The Future of Accelerator Physics: The Tamura Symposium, edited by T. Tajima, AIP Conf. Proc. No. 356 (AIP, New York, 1996); see D. V. Neuffer and R. B. Palmer, in ibid., p. 344.

[32] Beam Dynamics and Technology Issues for $\mu^{+} \mu^{-}$Colliders at the 9th Advanced ICFA Beam Dynamics Workshop (Ref. [11]).

[33] Proceedings of the 1996 DPF/DPB Summer Study on High-Energy Physics, edited by D. G. Cassel, L. T. Gennari, and R. H. Siemann (Stanford Linear Accelerator Center, Menlo Park, CA, 1997).

[34] Future High Energy Colliders, edited by Z. Parsa, AIP Conf. Proc. No. 397 (AIP, New York, 1997).

[35] Workshop on Muon Colliders, Berkeley, California (unpublished).

[36] Proceedings of the 1997 Particle Accelerator Conference, Vancouver, 1997 (IEEE, Piscataway, NJ, 1998); http:// www.triumf.ca/pac97/papers/

[37] Mini-Workshop on the Muon Collider Lattice, Upton, New York (unpublished).

[38] Mini-Workshop on Ionization Cooling, Batavia, Illinois (unpublished).

[39] Workshop of Physics at the First Muon Collider and at the Front End of the Muon Collider (Ref. [14]); http: //www.fnal.gov/projects/muon_collider/physics/talks.html

[40] Physics Potential and Development of $\mu-\mu$ Colliders (Ref. [4]).

[41] Muon Collider Collaboration Meeting, Gulf Shores, AL, 1998 (unpublished).

[42] Mini-Workshop on Ionization Cooling, Upton, New York (unpublished).

[43] R. B. Palmer, A. Sessler, and A. Tollestrup, in Proceedings of the 1996 DPF/DPB Summer Study on High-Energy Physics (Ref. [33]), p. 203; ftp://ftp.mumu.bnl.gov/pub/ documents/aba_new.ps

[44] The $\mu^{+} \mu^{-}$Collider Collaboration, BNL-52503, FERMILAB-Conf-96/092, LBNL-38946, 1996; http:// www.cap.bnl.gov/mumu/book.html

[45] V. Barger, M. Berger, J. Gunion, and T. Han, Phys. Rep. 286, 1 (1997); http://xxx.lanl.gov/hep-ph/9602397 
[46] J. Gunion, Report No. UCD-98-5, 1997; http:// xxx.lanl.gov/hep-ph/9802258

[47] See the collection of articles at the URL http://www. fnal.gov/projects/muon_collider/physics/physics.html; http://puhep1.princeton.edu/mumu/physics/

[48] R. B. Palmer et al., Nucl. Phys. B (Proc. Suppl.) 51A, 61 (1996).

[49] R. B. Palmer et al., in Beam Dynamics and Technology Issues for $\mu^{+} \mu^{-}$Colliders at the 9th Advanced ICFA Beam Dynamics Workshop (Ref. [11]); http://xxx.lanl.gov/ ps/acc-phys/960201

[50] R. B. Palmer and J.C. Gallardo, in Techniques and Concepts of High Energy Physics IX, edited by $\mathrm{T}$. Ferbel (Plenum, New York, 1997), p. 183; ftp:// ftp.mumu.bnl.gov/pub/documents/master_stcroix.ps

[51] R.B. Palmer and J.C. Gallardo, in Critical Problems in Physics, edited by V.L. Fitch, D. R. Marlow, and M. A.E. Dementi (Princeton University Press, Princeton, NJ, 1997), p. 247; http://xxx.lanl.gov/ps/physics/9702016

[52] R. B. Palmer, in Proceedings of the Particle Accelerator Conference, Vancouver, 1997 (Ref. [36]), p. 286; http:// www.triumf.ca/pac97/papers/pdf/6B002.PDF

[53] R. B. Palmer, BNL-65241, CAP-201-MUON-98C, 1998; http://xxx.lanl.gov/ps/physics/9802005

[54] R. B. Palmer, in Physics Potential and Development in $\mu-\mu$ Colliders (Ref. [4]), p. 183; http://xxx.lanl.gov/ps/ physics/9802002

[55] R. Palmer, A. Sessler, A. Tollestrup, and J. Gallardo, in Proceedings of the European Particle Accelerator Conference, Stockholm, Sweden, 1998 (Ref. [5]); http:// xxx.lanl.gov/ps/physics/9807006

[56] A.G. Ruggiero, in Advanced Accelerator Concepts (Ref. [1]), p. 958.

[57] S. Geer, in Workshop on Physics at the First Muon Collider and at the Front End of the Muon Collider (Ref. [39]), p. 384; http://www.fnal.gov/projects/ muon_collider/talks/steves_nu_talk.ps

[58] S. Geer, in International Workshop on JHF Science (KEK, Tsukuba, 1998); http://www.fnal.gov/projects/ muon_collider/talks/sg_nu_jhf98.ps

[59] S. Geer, Phys. Rev. D 57, 6989 (1998).

[60] R. Mohapatra, in Workshop on Physics at the First Muon Collider and at the Front End of the Muon Collider (Ref. [39]), p. 358; http://xxx.lanl.gov/ps/hep-ph/ 9711444

[61] D. A. Harris and K. S. McFarland, in Workshop on Physics at the First Muon Collider and at the Front End of the Muon Collider (Ref. [39]), p. 376; http://xxx.lanl.gov/ps/ hep-ex/9804010

[62] B. J. King, in Workshop on Physics at the First Muon Collider and at the Front End of the Muon Collider (Ref. [39]), p. 334; http://pubweb.bnl.gov/people/bking/ nufnal97.ps

[63] B. J. King, in Physics Potential and Development of $\mu-\mu$ Colliders (Ref. [4]), p. 132; http://pubweb.bnl.gov/people/ bking/sf97.ps

[64] B. J. King, BNL Report No. CAP-224-MUON-98C, 1998; http://pubweb.bnl.gov/people/bking/pr98.ps

[65] J. Yu and A. V. Kotwal, in Workshop on Physics at the First Muon Collider and at the Front End of the Muon Collider (Ref. [39]), p. 398.
[66] D. Cline and D. Neuffer, in High Energy Physics (Ref. [17]), p. 846; in Physics Potential and Development of $\mu^{+} \mu^{-}$Colliders: Second Workshop (Ref. [2]), p. 10.

[67] V. Barger, M. S. Berger, J. F. Gunion, and T. Han, Phys. Rev. Lett. 75, 1462 (1995).

[68] E. Richter-Was, D. Froidevaux, F. Gianotti, L. Poggioli, D. Cavalli, S. Resconi, Report No. CERN-TH-96-111, 1996.

[69] C. T. Hill, Phys. Lett. B 345, 483 (1995); K. Lane and E. Eichten, Phys. Lett. B 352, 382 (1995); K. Lane, Phys. Rev. D 54, 2204 (1996).

[70] R. Casalbuoni et al., Phys. Lett. B 285, 103 (1992).

[71] P. Bhat and E. Eichten, Fermilab Report No. FERMILAB-CONF-98/072; K. Lane, in Workshop on Physics at the First Muon Collider and at the Front End of the Muon Collider (Ref. [39]); http:// xxx.lanl.gov/ps/hep-ph/9810385; J. Womersley, Workshop on Physics at the First Muon Collider and at the Front End of the Muon Collider (Ref. [39]), p. 754 (1998); D. Dobrescu and C. T. Hill, Fermilab Report No. FERMILAB-PUB-97/409-T, http://xxx.lanl.gov/ ps/hep-ph/9712319; R. Casalbuoni et al., in Workshop on Physics at the First Muon Collider and at the Front End of the Muon Collider (Ref. [39]), http://xxx.lanl.gov/ ps/hep-ph/9801243; J. F. Gunion, Report No. UCD-98-5, http://xxx.lanl.gov/ps/hep-ph/9802258; R. Casalbuoni et al., Report No. UCD-98-13.

[72] J.F. Gunion, Int. J. Mod. Phys. A13, 2277 (1997), and references therein.

[73] J.L. Feng, J.F. Gunion, and T. Han, http://xxx.lanl.gov/ ps/hep-ph/9711414

[74] M. DeMarteau and T. Han, in Workshop on Physics at the First Muon Collider and at the Front End of the Muon Collider (Ref. [39]); http://xxx.lanl.gov/ps/hep-ph/ 9801407

[75] J. Erler and P. Langacker, Eur. Phys. J. C 3, 90 (1998); http://pdg/lbl.gov

[76] V. Barger, M. S. Berger, J.F. Gunion, and T. Han, Phys. Rev. D 56, 1714 (1997); Phys. Rev. Lett. 78, 3991 (1997).

[77] S. Parke, http://xxx.lanl.gov/ps/hep-ph/9802279

[78] See, e.g., M. Carena and S. Protopopescu, in Workshop on Physics at the First Muon Collider and at the Front End of the Muon Collider (Ref. [39]).

[79] H. Baer, C. Chen, C. Kao, and X. Tata, Phys. Rev. D 52, 1565 (1995).

[80] V. Barger, M.S. Berger, and T. Han, University of Wisconsin-Madison Report No. MADPH-98-1036; http:/ /xxx.lanl.gov/ps/hep-ph/9801410

[81] I. Hinchliffe, in Workshop on Physics at the First Muon Collider and at the Front End of the Muon Collider (Ref. [39]).

[82] F. Paige, in Workshop on Physics at the First Muon Collider and at the Front End of the Muon Collider (Ref. [39]).

[83] V. Barger, M. S. Berger, J.F. Gunion, and T. Han, in Proceedings of the Symposium on Physics Potential and Development of $\mu^{+} \mu^{-}$Colliders, San Francisco, CA, 1995, edited by D. Cline and D. Sanders [Nucl. Phys. B (Proc. Suppl.) 51A, 13 (1996)].

[84] J. Lykken, http://xxx.lanl.gov/ps/hep-ph/9803427 
[85] S. Godfrey, http://xxx.lanl.gov/ps/hep-ph/9802212

[86] V. Barger, M.S. Berger, J.F. Gunion, and T. Han, Phys. Rev. D 55, 142 (1997).

[87] W. J. Marciano, in Workshop on Physics at the First Muon Collider and at the Front End of the Muon Collider (Ref. [39]), p. 58; W. Molzon, ibid., p. 152.

[88] C. Ankenbrandt and S. Geer, in Workshop on Physics at the First Muon Collider and at the Front End of the Muon Collider (Ref. [39]), p. 3.

[89] Kingman Cheung, in Workshop on Physics at the First Muon Collider and at the Front End of the Muon Collider (Ref. [39]); http://xxx.lanl.gov/ps/hep-ph/9802219

[90] J. Norem et al., in Proceedings of the Particle Accelerator Conference, Vancouver, Canada, 1997 (Ref. [36]), p. 399; http://www.triumf.ca/pac97/papers/pdf/4W025.PDF

[91] KAON Factory Proposal, TRIUMF Internal Publication, September 1985 (unpublished).

[92] BNL Report No. BNL-60678, 1994.

[93] See Ref. [44], Chap. 3.

[94] C. Ankenbrandt and R. Noble, in Workshop on Physics at the First Muon Collider and at the Front End of the Muon Collider (Ref. [39]), p. 3; http://www-lib.fnal.gov/archive/ 1998/conf/Conf-98-074.html

[95] M. Blaskiewicz et al., in Proceedings of the 1995 Particle Accelerator Conference, Dallas, Texas (IEEE, Piscataway, NJ, 1996), p. 383.

[96] T. Roser, in Beam Dynamics and Technology Issues for $\mu^{+} \mu^{-}$Colliders at the 9th Advanced ICFA Beam Dynamics Workshop (Ref. [11]), p. 47; ftp://ftp.mumu.bnl.gov/ pub/documents/roser.ps

[97] L. Ahrens et al., in Proceedings of the Particle Accelerator Conference, Vancouver, Canada, 1997 (Ref. [36]), p. 89; http://www.triumf.ca/pac97/papers/pdf/7P001.PDF

[98] T. Roser, in Proceedings of the Workshop on Space Charge Physics in High Intensity Hadron Rings, Shelter Island, New York, 1998 (to be published).

[99] Y. Cho et al., in Beam Dynamics and Technology Issues for $\mu^{+} \mu^{-}$Colliders at the 9th Advanced ICFA Beam Dynamics Workshop (Ref. [11]), p. 31; ftp:// ftp.mumu.bnl.gov/pub/documents/harkay.ps

[100] S.D. Holmes, Fermilab Report No. FERMILAB-TM2021, 1997; http://www-lib.fnal.gov/archive/1997/tm/ c-TM-2021.pdf

[101] Fermilab Report No. FERMILAB-Pub-96/046, 1996; http://www-lib/fnal/gov/archive/1996/pub/Pub-96046.html

[102] K. Y. Ng and Z. Qian, Fermilab Report No. FERMILABConf-98-116, 1998; http://www-lib.fnal.gov/archive/1998/ conf/Conf-98-116.html

[103] J.E. Griffin, Fermilab Report No. FERMILAB-FN-669, 1998; http://www-lib.fnal.gov/archive/1998/fn/FN-669. html

[104] C. Ankenbrandt et al., Phys. Rev. ST Accel. Beams 1, 030101 (1998); http://www-lib.fnal.gov/archive/1998/pub/ Pub-98-006.html

[105] J. Norem et al., Report No. ANL-HEP-CP-98-32, 1998; J. Norem et al., in Proceedings of the Particle Accelerator Conference, Vancouver, Canada, 1997 (Ref. [36]), p. 396; http://www.triumf.ca/pac97/papers/pdf/4W024.PDF

[106] D. Trbojevic et al., in Proceedings of the Particle Accelerator Conference, Vancouver, Canada, 1997 (Ref. [36]), p. 1030; http://www.triumf.ca/pac97/papers/pdf/7W017. PDF

[107] J.E. Griffin, K.Y. Ng, Z.B. Qian, and D. Wildman, Fermilab Report No. FERMILAB-FN-661, 1997; http:// www-lib.fnal.gov/archive/1997/fn/FN-661.html

[108] J. E. Griffin et al., IEEE Trans. Nucl. Sci. NS-30, 3502 (1983).

[109] J. M. Brennan and M. M. Blaskiewicz, in Proceedings of the Workshop on Space Charge Physics in High Intensity Hadron Rings, Shelter Island, New York, 1998 (Ref. [98]).

[110] N. Mokhov, R. Noble, and A. Van Ginneken, in Beam Dynamics and Technology Issues for $\mu^{+} \mu^{-}$Colliders at the 9th Advanced ICFA Beam Dynamics Workshop (Ref. [11]), p. 61; http://www-lib.fnal.gov/archive/1996/ conf/Conf-96-006.html

[111] N. V. Mokhov and A. Van Ginneken, Fermilab Report No. FERMILAB-Conf-98/041, 1998; http://www-lib.fnal.gov/ 1998/archive/conf/Conf-998-041.html

[112] D. Ehst, N. V. Mokhov, R. J. Noble, and A. Van Ginneken, in Proceedings of the Particle Accelerator Conference, Vancouver, Canada, 1997 (Ref. [36]), p. 393; http:// www.triumf.ca/pac97/papers/pdf/4W023.PDF

[113] H. Takahashi, Y. An, X. Chen, and M. Nomura, in Proceedings of the Particle Accelerator Conference, Vancouver, Canada, 1997 (Ref. [36]), p. 402; http:// www.triumf.ca/pac97/papers/pdf/4W027.PDF

[114] S. H. Kahana, Y. Pang, and T. J. Schlagel, in Proceedings of Heavy Ion Physics at the AGS-HIPAGS '93, edited by G.S.F. Stephans et al. (unpublished) (BNL Internal Report No. BNL-48888); D. Kahana and Y. Torun, BNL Report No. BNL-61983, 1995; http://bnlnth.phy.bnl.gov/ arc/arc.html; http://www-pat.fnal.gov/muSim/ARC.html

[115] N. V. Mokhov, Fermilab Report No. FERMILAB-FN-628, 1998; http://www-ap.fnal.gov/MARS/

[116] N. V. Mokhov and S. I. Striganov, Fermilab Report No. FERMILAB-Conf-98-053, 1998; http://www-lib.fnal.gov/ archive/1998/conf/Conf-98-053.html

[117] J. Ranft, Report No. INFN-AE-97-45, 1997; http:// preprints.cern.ch/cgi-bin/setlink?base=preprint\&categ= scan\&id=SCAN-9711078

[118] Experiment E-910 at BNL-AGS; http://www.nevis. columbia.edu/heavyion/e910/

[119] Hiroyoshi Hiejima, heijima@nevis1.columbia.edu (private communication).

[120] R. B. Palmer et al., in Physics Potential and Development of $\mu^{+} \mu^{-}$Colliders: Second Workshop (Ref. [2]), p. 108; ftp://ftp.mumu.bnl.gov/pub/documents/monte1.ps

[121] J. Lettry et al., in Proceedings of ICANS-XIII, 1995, edited by G. S. Bauer and R. Bercher (Paul Scherrer Institute, Villigen, Switzerland, 1995), PSI-Proceedings 95-02; http://puhep1.princeton.edu/mumu/target/lettry/1.html

[122] C. Johnson, http://nicewww.cern.ch/ cdj/public/mumutarg/

[123] L. Ni and G. Bauer, PSI preprint, 1998; http:// puhep1.princeton.edu/mumu/target/bauer/1.html

[124] C. Lu and K.T. McDonald, Report No. Princeton $/ \mu \mu /$ 97-3, 1998; http://www.hep.princeton.edu/mumu/mumu97-3.ps

[125] R. Weggel, BNL Report No. CAP-220-MUON-98R, 1998.

[126] C. Lu and K.T. McDonald, Report No. Princeton/ $\mu \mu$ / 98-10, 1998; http://www.hep.princeton.edu/mumu/mumu98-10.ps 
[127] B. J. King, N. V. Mokhov, and R. Weggel (unpublished); http://pubweb.bnl.gov/people/bking/talks.html

[128] R. Weggel, BNL Report No. MU-015, 1997.

[129] J.R. Miller et al., IEEE Trans. Magn. 30, 1563 (1994).

[130] J. Broere et al., in Proceedings of the XIX International LINAC Conference, Chicago, Illinois, ANL-98/ 28 (Argonne National Laboratory, Argonne, 1998); http://www.aps.anl.gov/conferences/LINAC98/papers/ TH4004.pdf

[131] H. Gaiser et al., in Proceedings of the 1999 Particle Accelerator Conference (PAC99), New York (to be published); http://ftp.pac99.bnl.gov/Papers/Wpac/FRA70.pdf

[132] R. B. Palmer and J. C. Gallardo (unpublished).

[133] See Ref. [44], p. 220.

[134] K. Assamagan et al., Phys. Lett. B 335, 231 (1994).

[135] E.P. Wigner, Ann. Math. 40, 149 (1939).

[136] E. P. Wigner, Rev. Mod. Phys. 29, 255 (1957).

[137] B. Norum and R. Rossmanith, Nucl. Phys. B (Proc. Suppl.) 51A, 191 (1996).

[138] J. Alessi et al., proposal to the BNL AGS, 1998; http:// puhep1.princeton.edu/mumu/target/targetprop.ps

[139] A. A. Mikhailichenko and M.S. Zolotorev, Phys. Rev. Lett. 71, 4146 (1993).

[140] A. Hershcovitch, AGS/AD Tech. Note No. 413, BNL, 1996; in Proceedings of the Particle Accelerator Conference, Vancouver, Canada, 1997 (Ref. [36]), p. 1792; http:/ /www.triumf.ca/pac97/papers/pdf/4V024.PDF

[141] P. Chen, Z. Huang, and R. Ruth, in Advanced Accelerator Concepts: Sixth Annual Conference (Ref. [29]), p. 646.

[142] P. H. Sandler, S. A. Bogacz, and D. B. Cline, in Potential and Development of $\mu^{+} \mu^{-}$Colliders: Second Workshop (Ref. [2]), p. 146.

[143] Reference [44], Chap. 6.

[144] R.C. Fernow and J.C. Gallardo, Phys. Rev. E 52, 1039 (1995); ftp://ftp.mumu.bnl.gov/pub/documents/ stochastic.ps

[145] R.C. Fernow and J.C. Gallardo, in Physics Potential and Development of $\mu^{+} \mu^{-}$Colliders: Second Workshop (Ref. [2]), p. 170.

[146] Particle Data Group, Eur. Phys. J. C 3, 138 (1998); http:// pdg/lbl.gov

[147] A. Van Ginneken, Nucl. Instrum. Methods Phys. Res., Sect. A 362, 213 (1995).

[148] R. Fernow (unpublished); http://pubweb.bnl.gov/people/ fernow/readme.html; http:/www-pat.fnal.gov/muSim/ icool.html

[149] R. Fernow, in Proceedings of the 1999 Particle Accelerator Conference (PAC99), New York (to be published); http://pubweb.bnl.gov/people/fernow/reports/icoolp99.ps

[150] H. Kirk, in Ref. [42].

[151] GEANT Manual, v.3.2.1, CERN program Library W5013 (CERN, Geneva, 1994); http://wwwinfo.cern.ch/asdoc/ geant_html3/geantall.html

[152] P. Lebrun, in Ref. [42].

[153] C. M. Celata, A. M. Sessler, P.B. Lee, B. A. Shadwick, and J.S. Wurtele, in Proceedings of the European Particle Accelerator Conference, Stockholm, 1998 (Ref. [5]).

[154] R. Palmer, http://pubweb.bnl.gov/people/palmer/course/ 6cool.ps
[155] G. Silvestrov, in Ref. [28].

[156] B. Bayanov et al., Nucl. Instrum. Methods 190, 9 (1981).

[157] C. D. Johnson, Hyperfine Interact. 44, 21 (1988).

[158] G. Silvestrov, in Beam Dynamics and Technology Issues for $\mu^{+} \mu^{-}$Colliders at the 9th Advanced ICFA Beam Dynamics Workshop (Ref. [11]), p. 168; ftp:// ftp.mumu.bnl.gov/pub/documents/greg.ps

[159] V. Balbekov and A. Van Ginneken, in Ref. [38].

[160] V. Balbekov and A. Van Ginneken, in Physics Potential and Development of $\mu-\mu$ Colliders (Ref. [4]), p. 310.

[161] R. B. Palmer, BNL Report No. BNL-CAP-MU-022, 1998; http://pubweb.bnl.gov/people/palmer/notes/whyalt.ps

[162] R. C. Fernow, J. C. Gallardo, H. G. Kirk, and R. B. Palmer (to be published); http://pubweb.bnl.gov/people/fernow/ reports/asol.ps

[163] M. Leupold et al., in Proceedings of the 7th International Conference on Magnet Technology [IEEE Trans. Magn. 17, 1779-1782 (1981)]; H.-J. Schneider-Muntau, and J. Vallier, IEEE Trans. Magn. 24, 1067-1069 (1988).

[164] See Ref. [129]; R. Weggel et al., in Proceedings of the 14th International Conference on Magnet Technology [IEEE Trans. Magn. 32, 2474 (1996)].

[165] J. C. Gallardo, R. C. Fernow, and R. B. Palmer, in Physics Potential and Development of $\mu-\mu$ Colliders (Ref. [4]), p. 282.

[166] J. Norem, Phys. Rev. ST Accel. Beams 2, 054001 (1999).

[167] D. Neuffer, in Physics Potential and Development of $\mu-\mu$ Colliders (Ref. [4]), p. 270.

[168] D. Neuffer and A. Van Ginneken, in Ref. [42].

[169] D. Neuffer and W. Wan, in Ref. [38].

[170] N. Holtkamp, FNAL Internal Note, 1999; http://wwwmucool.fnal.gov/mcnotes/muc0013.ps; D. Li et al., http:// www.aps.anl.gov/conferences/LINAC98/papers/MO4089. pdf

[171] C. N. Ankenbrandt et al., Report No. Fnal-P904, 1998; http://www.fnal.gov/projects/muon_collider/

[172] S. Geer, in International Workshop on JHF Science (JHF98) (KEK, Tsukuba, Japan, 1998); http:// fnalpubs.fnal.gov/archive/1998/conf/Conf-98-136.html

[173] C. Lu, K. T. McDonald, E.J. Prebys, and S.E. Vahsen, Report No. Princeton/ $\mu \mu / 97-8$, 1998; http:// www.hep.princeton.edu/mumu/mumu-97-8.ps

[174] F. Mills, fredmills@aol.com (private communication).

[175] B.J. King, BNL Report No. BNL-CAP-MU-023, 1998; http://pubweb.bnl.gov/people/bking/magnet.ps

[176] D. Neuffer, Nucl. Instrum. Methods Phys. Res., Sect. A 384, 263 (1997).

[177] D. V. Neuffer, in Beam Dynamics and Technology Issues for $\mu^{+} \mu^{-}$Colliders at the 9th Advanced ICFA Beam Dynamics Workshop (Ref. [11]), p. 315; ftp:// ftp.mumu.bnl.gov/pub/documents/aclmntk.ps

[178] D. Summers, D. Neuffer, Q.-S. Shu, and E. Willen, in Proceedings of the Particle Accelerator Conference, Vancouver, Canada, 1997 (Ref. [36]), p. 624; http:// www.triumf.ca/pac97/papers/pdf/3PC004.PDF

[179] D. J. Summers, in Proceedings of the DPF/DPB Summer Study on High-Energy Physics (Ref. [33]), p. 238.

[180] TESLA Collaboration, Report No. TESLA 95-01, 1995. 
[181] Z. D. Farkas et al., SLAC Report No. SLAC-PUB-1435; in Proceedings of the 9th International Conference on High Energy Accelerators, Stanford, California, 1974 (Stanford Linear Accelerator Center, Menlo Park, CA, 1974), p. 576.

[182] E. Willen, BNL Magnet Note 555-31, 1996; also in Proceedings of the DPF/DPB Summer Study on HighEnergy Physics (Ref. [33]), p. 246.

[183] Allied Signal, Amorphous Metals Division, 6 Eastmans Road, Parsippany, NJ 07054.

[184] Magnetics, Division of Spang \& Company, 900 East Butler Road, Butler, PA 16003.

[185] I. Campisi, campisi@jlab.org (private communication).

[186] A. Mosnier and O. Napoly, Int. J. Mod. Phys. A (Proc. Suppl.) 2B, 963 (1993).

[187] A. Novokhatski and A. Mosnier, in Proceedings of the Particle Accelerator Conference, Vancouver, Canada, 1997 (Ref. [36]), p. 467; http://www.triumf.ca/pac97/ papers/pdf/8W013.PDF

[188] V. Balakin, A. Novokhatsky, and V. Smirnov, in Proceedings of the 12th International Conference on High Energy Accelerators, edited by F. T. Cole and R. Donaldson (Fermilab, Batavia, IL, 1983), p. 119.

[189] A. Chao, Physics of Collective Beam Instabilities in High Energy Accelerators (Wiley, New York, 1993).

[190] K. L. Brown and J. Spencer, IEEE Trans. Nucl. Sci. NS28, 2568 (1981).

[191] K.L. Brown, SLAC Report No. SLAC-PUB-4811, 1988.

[192] K. L. Brown, J. J. Murray, and T.H. Fieguth, SLAC Report No. SLAC-PUB-4219, 1987; http://ccdb1.kek.jp/ cgi-bin/img_index?8706254

[193] O. Napoly, CLIC Note 227 (CERN), 1994.

[194] C. Johnstone et al., in Physics Potential and Development of $\mu-\mu$ Colliders (Ref. [4]), p. 209.

[195] C. J. Johnstone and N. V. Mokhov, in Proceedings of the DPF/DPB Summer Study on High-Energy Physics (Ref. [33]), p. 226; Fermilab Report No. FERMILABConf-96-366, 1996; http://www-lib.fnal.gov/archive/1996/ conf/Conf-96-366.html

[196] A. Drozhdin et al., in Physics Potential and Development of $\mu-\mu$ Colliders (Ref. [4]), p. 242; Fermilab Report No. FERMILAB-Conf-98/042, 1998; http://www-lib.fnal.gov/ archive/1998/conf/Conf-98-042.html

[197] M. Donald et al., in Proceedings of the 1993 Particle Accelerator Conference, Washington, DC, 1993 (IEEE, Piscataway, NJ, 1993), p. 131.

[198] W. Wan and C. Johnstone, in Ref. [37].

[199] K.-Y. Ng, S. Y. Lee, and D. Trbojevic, Fermilab Report No. FNAL-FN595, 1992.

[200] S. Y. Lee, K. Y. Ng, and D. Trbojevic, Phys. Rev. E 48, 3040 (1993).

[201] M. Berz, "COSY INFINITY, V. 8. User's Guide and Reference Manual," MSU Internal Report (unpublished); http://www.beamtheory.nscl.msu.edu/cosy/

[202] S. Ohnuma, ohnuma@uh.edu (private communication).

[203] N. Merminga and K. Y. Ng, Fermilab Report No. FN-493, 1988.

[204] Y.T. Yan, yan@slac.stanford.edu (private communication).

[205] L. Schachinger and R. Talman, Part. Accel. 22, 35 (1987).
[206] G. W. Foster and N. V. Mokhov, in Physics Potential and Development of $\mu^{+} \mu^{-}$Colliders: Second Workshop (Ref. [2]), p. 178.

[207] M. Furman, BF-19/CBP-Note-169/LBL-38563, 1996.

[208] P. Chen, Nucl. Phys. B (Proc. Suppl.) 51A, 179 (1996).

[209] W.-H. Cheng, A. M. Sessler, and J. Wurtele, in Beam Dynamics and Technology Issues for $\mu^{+} \mu^{-}$Colliders at the 9th Advanced ICFA Beam Dynamics Workshop (Ref. [11]), p. 206.

[210] K. Y. Ng, in Beam Dynamics and Technology Issues for $\mu^{+} \mu^{-}$Colliders at the 9th Advanced ICFA Beam Dynamics Workshop (Ref. [11]), p. 224.

[211] N. V. Mokhov and S. I. Striganov, in Beam Dynamics and Technology Issues of $\mu^{+} \mu^{-}$Colliders at the 9th Advanced ICFA Beam Dynamics Workshop (Ref. [11]), p. 234; http:/ /www-lib.fnal.gov/archive/1996/conf/Conf-96-011.html

[212] E. Willen et al., in Proceedings of the Particle Accelerator Conference, Vancouver, Canada, 1997 (Ref. [36]), p. 3362; http://www.triumf.ca/pac97/papers/pdf/3P010.PDF

[213] T. Roser et al. (unpublished); http://www.ags.bnl.gov/ $\sim$ roser/cdr07034/cdr07034.html

[214] E. Willen, willen@bnl.gov (private communication).

[215] N. V. Mokhov, Fermilab Report No. FERMILAB-Conf96/367, 1996; http://www-lib.fnal.gov/archive/1996/conf/ Conf-96-367.html

[216] B. J. King, BNL Report No. BNL-CAP-MU-024; http:// pubweb.bnl.gov/people/bking/1996/

[217] B. J. King, in Ref. [35].

[218] B. J. King, BNL Report No. CAP-162-MUON-97R, 1997.

[219] B.J. King (unpublished); http://pubweb.bnl.gov/people/ bking/nurad_aps98/

[220] Particle Data Group, Eur. Phys. J. C 3, 163 (1998); http:// pdg/lbl.gov

[221] C. Johnstone and N. Mokhov, in Proceedings of the Particle Accelerator Conference, Vancouver, Canada, 1997 (Ref. [36]), p. 414; http://www.triumf.ca/pac97/ papers/pdf/4W030.PDF

[222] K. V. Lotov, A. N. Skrinsky, and A. V. Yashin, Budker Report No. INP 98-41.

[223] I. Stumer et al., in Proceedings of the 1996 DPF/DPB Summer Study on High-Energy Physics (Ref. [33]), p. 463.

[224] N. V. Mokhov, Nucl. Phys. B (Proc. Suppl.) 51A, 210 (1996); http://fnalpubs.fnal.gov/archive/1996/conf/Conf96-062.html

[225] Reference [44], Sec. 8.5.3.

[226] I. Ginzburg, Nucl. Phys. B (Proc. Suppl.) 51A, 186 (1996); http://xxx.lanl.gov/ps/hep-ph/9601273

[227] S. Geer, in Proceedings of the 1996 DPF/DPB Summer Study on High-Energy Physics (Ref. [33]).

[228] The SLD Collaboration, Nuovo Cimento Soc. Ital. Fis. 109A, 1027 (1996).

[229] E. Gatti and P. Rehak, Nucl. Instrum. Methods 225, 608 (1984).

[230] S. I. Parker, C. J. Kenney, and J. Segal, Nucl. Instrum. Methods Phys. Res., Sect. A 395, 328 (1997).

[231] S. Geer and J. Chapman, in Proceedings of the 1996 DPF/ DPB Summer Study on High-Energy Physics (Ref. [33]); Fermilab Report No. FERMILAB-CONF-96-375.

[232] Reference [44], Sec. 9.4.2.

[233] M. Atac, Fermilab Report No. FERMILAB-CONF96-401, 1996; in Proceedings of the 1996 DPF/DPB 
Summer Study on High-Energy Physics (Ref. [33]), [234] R. Raja, Fermilab Muon Collider Note MC-042, 1997. p. 478. 